Teorema de Radó para campos vetoriais localmente resolúveis

Manuel Francisco Zuloeta Jimenez 



\title{
Teorema de Radó para campos vetoriais localmente resolúveis ${ }^{1}$
}

\author{
Manuel Francisco Zuloeta Jimenez
}

Orientador: Prof. Dr. Evandro Raimundo da Silva

Dissertação apresentada ao Instituto de Ciências Matemáticas e de Computação - ICMC-USP, como parte dos requisitos para obtenção do título de Mestre em Ciências - Matemática.

USP - São Carlos

Julho de 2010

\footnotetext{
${ }^{1}$ Este trabalho teve suporte financeiro da CAPES
} 

O futuro não pode ser previsto, mas pode ser inventado. É a nossa habilidade de inventar o futuro que nos dá esperança para fazer de nós o que somos.

Dennis Gabor 



\section{Agradecimentos}

Tentarei expressar em poucas linhas minha gratidão às pessoas que de alguma forma estiveram presentes e que muito me ajudaram a escrever as páginas de mais esta vitória.

Inicialmente gostaria de expressar minha eterna gratidão a Deus pelo amor, pela força que me impulsiona a lutar pelos meus objetivos e por me ter permitido, sempre com muita fé, ultrapassar todos os obstáculos.

Agradeço à minha família que sempre esteve presente para me dar suporte de forma incondicional em todos os aspectos possíveis durante meus estudos, mesmo distante. Aos meus pais, Juan e Gladis, pela dedicação, pela educação e pelo esforço para comigo e meus irmãos. Aos meus irmãos, Fernando, Elvis, Angel, Jose, Clara e Paola, pelo incentivo, carinho e compreensão ao longo destes anos de dedicação à Matemática. Aos meus sobrinhos, Crisby, Diego, Carmen, Dayana e Josue, obrigado por fazer de minha vida mais feliz. Amo todos vocês!

Agradeço ao meu orientador, Prof. Evandro Raimundo da Silva, pelo apoio, pela amizade, pela dedicação e por estar sempre disposto em ajudar no que fosse preciso. Pelo constante incentivo, por acreditar sempre no meu trabalho, por me ajudar a trilhar este caminho difícil, mas que no final, é compensador, muito obrigado!

Agradeço aos professores do departamento de Matemática do ICMC, pelos ensinamentos, apoio, motivação, pela amizade e pelas aulas ministradas durante este período com as quais muito aprendi.

Agradeço aos colegas e amigos da USP e em especial, aqueles que estiveram sempre presentes: Nancy Chachapoyas, José Bravo, Luis Espinoza, Napoleon Caro, Norbil Cordova, Lizandro Sanchez e Walter Huaraca. Obrigado pelo apoio, pelas longas conversas, pelas horas de estudo, pelos dias de futebol e por todos os momentos inesquecíveis vividos.

Agradeço a todos meus amigos que conheço há muito tempo e que sem dúvida tem um papel fundamental em toda na minha vida: Augusto, Juan, Eder, Raul, Marlenny, Carlos, Jose, Alex... Obrigado por todos os momentos divertidos, pelo exemplo de pessoas 
viii

íntegras que são, gigantes que me emprestam seu ombro para apoiar e ver mais longe.

Não poderia deixar de agradecer aos funcionários do ICMC por estarem sempre à disposição e tornarem nossa vivência mais agradável

Agradeço à CAPES, Coordenação de Aperfeiçoamento de Pessoal de Nível Superior, pelo suporte financeiro durante meus estudos.

Enfim, se for agradecer a cada um, não acabaria nunca, pois são tantas as pessoas especiais que passaram e marcaram esta jornada...

Mais uma vez a todos vocês muito obrigado!!! 


\section{Resumo}

Um resultado conhecido na teoria de variáveis complexas é o Teorema clássico de Radó que afirma que se uma função complexa $u$ é contínua em $\bar{D}(0,1)=\{z \in \mathbb{C} ;|z| \leq 1\}$ e holomorfa em $U=\{z \in \mathbb{C} ;|z|<1, u(z) \neq 0\}=D(0,1) \backslash u^{-1}(0)$, então é holomorfa em $D(0,1)=\{z \in \mathbb{C} ;|z|<1\}$. Diferentes provas e generalizações para este resultado foram dadas por muitos autores, ver por exemplo, [4], [7], [8], [10] and [13]. Em [7] J. Hounie e J.Tavares provaram um Teorema do tipo Radó no caso de soluções homogêneas de campos vetoriais localmente resolúveis com coeficientes suaves. Mais precisamente, eles provaram o seguinte teorema:

Seja L um campo vetorial com coeficientes suaves definido em um subconjunto aberto $\Omega \subset \mathbb{R}^{n+1}$ satisfazendo a condição $(P)$. Então L tem a propriedade de Radó.

O objetivo principal deste trabalho é apresentar um estudo detalhado deste resultado. Mas antes faremos um estudo geral da teoria que está por trás deste resultado, como teoria de distribuições, estruturas localmente integráveis, resolubilidade local, entre outros. A exposição de tais conteúdos não será longa, pois o intuito é apenas indicar o que é minimamente necessário para entender a prova deste resultado.

Palavras chaves: Teorema de Radó, estruturas localmente integráveis, resolubilidade local, Teorema de aproximação de Baouendi-Treves. 


\section{Abstract}

A known result in complex variables theory is the classical Radó's Theorem, which states that if a complex function $f$ is continuous in $\bar{D}(0,1)=\{z \in \mathbb{C} ;|z| \leq 1\}$ and holomorphic on $U=\{z \in \mathbb{C} ;|z|<1, f(z) \neq 0\}=D(0,1) \backslash f^{-1}(0)$ then it is holomorphic in $D(0,1)=\{z \in \mathbb{C} ;|z|<1\}$. Different proofs and generalizations of this result have been given by many authors, see e.g. [4], [7], [8], [10] and [13]. In [7] J. Hounie e J.Tavares proved a Radó type Theorem for homogeneous solutions of locally solvable vector fields with smooth coefficients. More precisely they proved the following theorem:

Let $L$ be a vector field with smooth coefficients in an open subset $\Omega \subset \mathbb{R}^{n+1}$ satisfying condition (P). Then $L$ has the property of Radó.

The main goal of this work is to present a detailed study of this theorem. But before we will do an overall study of the theory behind of this result such as theory of distributions, locally integrable structures, local solvability, among others. The presentation of such contents will not be long, since the purpose is only to indicate what is minimally necessary for understanding the proof of this result.

Keywords: Radó's Theorem, Locally integragle structures, local solvability, BaouendiTreves approximation Theorem. 


\section{Sumário}

Introdução 1

1 Resultados básicos sobre distribuições 3

1.1 Funções teste . . . . . . . . . . . . . . . . . . . . . . . . . . . 3

1.2 As distribuições . . . . . . . . . . . . . . . . . . . . . . . . . 10

1.2.1 Operações com distribuições . . . . . . . . . . . . . . . . . . . . 13

1.2.2 Igualdade e suporte de distribuições . . . . . . . . . . . . . . . . . . 18

2 Teorema de Mergelyan e teorema clássico de Radó 23

2.1 O Teorema de Mergelyan . . . . . . . . . . . . . . . . . . . . . . . 30

2.2 O Teorema clássico de Radó . . . . . . . . . . . . . . . . . . . . . . . . . . 39

3 Estruturas involutivas e Estruturas localmente integráveis 43

3.1 Campos vetoriais complexos sobre variedades . . . . . . . . . . . . . . . 43

3.2 Vetores tangentes complexos e 1-formas diferenciáveis . . . . . . . . . . . . 51

3.3 Estruturas localmente integráveis . . . . . . . . . . . . . . . . . . . . 60

3.4 O teorema de Frobenius e geradores locais . . . . . . . . . . . . . . . 70

3.5 Teorema de aproximação de Baouendi-Treves . . . . . . . . . . . . . . . . . 81

4 Campos de vetores localmente resolúveis $\quad 95$

4.1 Campos de vetores no plano . . . . . . . . . . . . . . . . . . . 95

4.2 Campos de vetores em várias variáveis . . . . . . . . . . . . . . . . 102

5 Teorema de Radó para campos vetoriais localmente resolúveis $\quad 111$

5.1 O resultado principal . . . . . . . . . . . . . . . . . . . . . . . 114

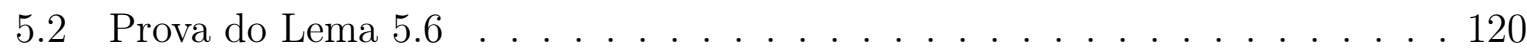




\section{Introdução}

A teoria de variáveis complexas é uma ferramenta muito importante para a matemática. Nos últimos anos têm surgido várias generalizações de resultados clássicos da teoria de variáveis complexas. Um resultado conhecido é o Teorema clássico de Radó o qual afirma que:

Se uma função complexa $u$, é continua em $\bar{D}(0,1)=\{z \in \mathbb{C} ;|z| \leq 1\}$ e holomorfa em $U=D(0,1) \backslash u^{-1}(0)$, então é holomorfa em $D(0,1)=\{z \in \mathbb{C} ;|z|<1\}$.

Este teorema implica facilmente que o mesmo resultado vale para funções de várias variáveis complexas. Diferentes provas e generalizações para este resultado foram dadas por muitos autores. Uma generalização para uma classe mais geral de funções foi dada por Rosay e Stout [10], eles estenderam o Teorema de Radó para CR-funções sobre hipersuperfícies de $\mathbb{C}^{n}$ estritamente pseudoconvexas e formularam um problema mais geral sobre campos vetoriais complexos. A solução deste problema foi dada por J.Hounie e J. Tavares [7] no caso de campos vetoriais complexos localmente resolúveis com coeficientes suaves definidos em um subconjunto aberto de $\mathbb{R}^{n+1}$. Para descrever brevemente o resultado, seja $L$ um campo vetorial complexo definido em um subconjunto aberto $\Omega \subset \mathbb{R}^{N}$,

Definição. Uma função $u \in C(\Omega)$ que satisfaz a equação $L u=0$ no sentido das distribuições no conjunto $\Omega \backslash u^{-1}(0)$ é chamada uma função de Radó para $L$. Dizemos que o campo vetorial $L$ tem a propriedade Radó, se toda função de Radó para $L$ é uma solução fraca de $L u=0$ em $\Omega$.

Definição. Seja $p \in \Omega$, dizemos que $L$ é localmente resolúvel em $p$ se existe uma vizinhança $U=U(p)$ tal que para toda $f \in C^{\infty}(\Omega)$ existe $u \in \mathcal{D}^{\prime}(\Omega)$ tal que $L u=f$ em $U$. Se $L$ é localmente resolúvel em todo ponto $p \in \Omega$, dizemos que $L$ é localmente resolúvel em $\Omega$.

Tendo presente que os campos vetoriais localmente resolúveis são conhecidos por serem caracterizados pela condição (P) introduzida por Nierenberg e Treves em [9], J.Hounie e J.Tavares provaram o seguinte teorema: 
Seja L um campo vetorial com coeficientes suaves definido em um subconjunto aberto $\Omega \subset \mathbb{R}^{n+1}$ satisfazendo a condição $(P)$. Então L tem a propriedade de Radó.

No caso em que $n=1$ e $L$ é o operador de Cauchy-Riemann o resultado se reduz ao Teorema clássico de Radó citado acima.

O objetivo principal da presente monografia é apresentar um estudo detalhado deste resultado. A prova deste resultado explora duas propriedades importantes dos campos vetoriais localmente resolúveis: o seu caráter essencialmente bidimensional (expressa pelo fato de que suas órbitas tem dimensão no máximo dois) e a propriedade da integrabilidade local que permite a redução do problema à versão clássica do Teorema de Radó no plano. Quando falamos das órbitas de um campo vetorial nos referimos às órbitas do campo no sentido de Sussmann cuja definição foi introduzida por Sussmann em [14]. Para uma melhor compreensão, esta monografia esta dividida em cinco capítulos

No Capítulo 1, apresentamos alguns resultados importantes sobre a teoria de distribuições, os quais serão utilizados nos capítulos posteriores. Para um tratamento detalhado dos tópicos apresentados, ver por exemplo, Rudin [11], Hounie [6] e Trèves [15].

No Capítulo 2, apresentamos um apanhado sobre funções holomorfas e funções harmônicas, principalmente mostraremos dois teoremas de grande importância para este trabalho, o Teorema de Mergelyam e o Teorema clássico de Radó. Para mais detalhes desta parte pode-se ver, Conway [2], Kaufman [8] e Rudin [12].

No Capítulo 3, vamos dar a definição de estrutura localmente integrável bem como algumas de suas propriedades mais importantes, mostraremos o Teorema de geradores locais o qual nos permitirá construir coordenadas e geradores locais adequados para o subfibrado vetorial $T^{\prime}$ e finalmente mostraremos o Teorema de aproximação de BaouendiTreves no caso em que $L$ é um campo vetorial definido em um subconjunto aberto de $\mathbb{R}^{2}$, $u$ é uma função contínua e temos uma única solução $Z$. Nossas principais referências para este capítulo são [1] e [3].

No Capítulo 4, vamos apresentar a definição de resolubilidade local de um campo vetorial, a condição $(\mathrm{P})$ e de forma rápida introduziremos a definição de órbitas de um campo vetorial no sentido de Sussmann. Um dos resultados importantes neste capítulo é que todo campo vetorial localmente resolúvel é localmente integrável. Para este capítulo nossas referências são [1], [3] e [14].

No Capítulo 5, fazemos uma exposição, em detalhes, do Teorema 2.1 de [7], que aqui aparece como Teorema 5.4. Uma observação a fazer é que neste capítulo assumimos que a condição $(\mathrm{P})$ é condição necessária e suficiente para a resolubilidade local de um campo vetorial. 


\section{$-\left[\begin{array}{c}\text { Captulu } \\ 1\end{array}\right]$ \\ Resultados básicos sobre distribuições}

Neste capítulo pretendemos abordar alguns resultados importantes sobre a teoria das distribuições, os quais serão utilizados nos capítulos posteriores. Iremos colocar as idéias de maneira direta com o objetivo apenas de estabelecer noções básicas, deixando claro que o leitor deverá possuir conhecimentos mínimos de análise real e teoria da medida. Alguns resultados serão somente enunciados sendo omitidas as suas demonstrações.

Para uma maior quantidade de detalhes pode-se consultar Rudin [11], Hounie [6] e Trèves [15].

Salvo menção explícita em contrário, ao longo deste capítulo, $\Omega$ representará um subconjunto aberto de $\mathbb{R}^{n}$.

\subsection{Funções teste}

Definição 1.1. Seja $f: \Omega \longrightarrow \mathbb{C}$, dizemos que $f$ é de classe $C^{k}$ (ou seja $f \in C^{k}(\Omega)$ ), se $\partial^{\alpha} f$ existe e é contínua até ordem $k$ isto é para $\alpha$ com $|\alpha| \leq k$.

Onde $\alpha=\left(\alpha_{1}, \alpha_{2}, \ldots, \alpha_{n}\right) \in \mathbb{Z}_{+}^{n}, \partial^{\alpha}=\partial_{x_{1}}^{\alpha_{1}} \ldots \partial_{x_{n}}^{\alpha_{n}}$ e $|\alpha|=\alpha_{1}+\alpha_{2}+\ldots+\alpha_{n}$. $C^{\infty}(\Omega)=\bigcap_{k \geq 0} C^{k}(\Omega)$

Definição 1.2. Seja $f: \Omega \longrightarrow \mathbb{C}$ uma função contínua, o conjunto

$$
\operatorname{supp}(f)=\overline{\{x \in \Omega ; f(x) \neq 0\}}
$$

é o suporte da função $f$.

Ou seja o suporte da função $f$ é o menor subconjunto fechado de $\Omega$ tal que $f \equiv 0$ em seu complementar. 
Observação 1.3. $x$ não pertence ao $\operatorname{supp}(f)$ se e somente se $u \equiv 0$ em uma vizinhança de $x$ em $\Omega$.

As funções de nosso principal interesse serão as de classe $C^{\infty}(\Omega)$ que possuem suporte compacto, para este propósito vamos dar a seguinte definição.

Definição 1.4. Chamaremos de espaço das funções teste ao conjunto

$$
C_{c}^{\infty}(\Omega)=\left\{f: \Omega \longrightarrow \mathbb{C} ; f \in C^{\infty}(\Omega) \text { e supp }(f) \text { é compacto em } \Omega\right\} .
$$

Exemplo 1.5. Um exemplo de uma função teste em $\mathbb{R}^{n}$, ou seja de uma função que pertence ao espaço $C_{c}^{\infty}\left(\mathbb{R}^{n}\right)$ é dada por

$$
\varphi(x)=g\left(1-|x|^{2}\right)=\left\{\begin{array}{cc}
p\left(\frac{1}{1-|x|^{2}}\right) e^{-\frac{1}{1-|x|^{2}}} & , \text { se }|x|<1 \\
0 & , \text { se }|x| \geq 1
\end{array}\right.
$$

onde $p$ é um polinômio qualquer em $\mathbb{R}$ e $g$ é uma função real definida da seguinte forma

$$
g(t)=\left\{\begin{array}{cc}
p\left(\frac{1}{t}\right) e^{-\frac{1}{t}}, & \text { se } t>0 \\
0, & \text { se } t \geq 0
\end{array}\right.
$$

\section{Observação 1.6.}

1) Pode-se observar que a funcão $\varphi$ do Exemplo 1.5 satisfaz: $\varphi \geq 0, \varphi(0)>0 e$ $\operatorname{supp}(\varphi) \subset \overline{B(0,1)}$. Mais ainda, multiplicando $\varphi$ por uma constante adequada obtemos uma nova função que além de satisfazer as condições anteriores, satisfaz também que $\int \varphi(x) d x=1$.

2) Seja $\varphi \in C_{c}^{\infty}\left(\mathbb{R}^{n}\right) \operatorname{com} \varphi \geq 0$ e supp $(\varphi) \subset \overline{B(0,1)}$, se $x_{0}$ é um ponto de $\mathbb{R}^{n}$ e $\epsilon>0$, então podemos considerar a seguinte função

$$
\varphi_{\epsilon}^{x_{0}}(x)=\frac{1}{\epsilon^{n}} \varphi\left(\frac{x-x_{0}}{\epsilon}\right)
$$

a qual satisfaz que $\operatorname{supp}\left(\varphi_{\epsilon}^{x_{0}}\right) \subset \overline{B\left(x_{0}, \epsilon\right)}$. Se $x_{0}=0, \varphi_{\epsilon}^{x_{0}}$ será denotada por $\varphi_{\epsilon}$.

Antes de enunciar o teorema seguinte vamos definir o que é uma função localmente integrável. 
Definição 1.7. Seja $f: \Omega \longrightarrow \mathbb{C}$ uma função Lebesgue mensurável, dizemos que $f$ é localmente integrável e escrevemos $f \in L_{\text {Loc }}^{1}(\Omega)$ se para cada compacto $K \subset \Omega$ temos que

$$
\int_{K}|f(x)| d x<\infty
$$

Teorema 1.8. Seja $\varphi \in C_{c}^{\infty}\left(\mathbb{R}^{n}\right)$ tal que $\int \varphi(x) d x=1, \varphi \geq 0, \operatorname{supp}(\varphi) \subset \overline{B(0,1)}$ e $f \in L_{\text {Loc }}^{1}\left(\mathbb{R}^{n}\right)$. Para cada $\epsilon>0$, definimos

$$
f * \varphi_{\epsilon}(x)=\int_{\mathbb{R}^{n}} f(x-\epsilon y) \varphi(y) d y=\int_{\mathbb{R}^{n}} f(y) \varphi_{\epsilon}(x-y) d y=\frac{1}{\epsilon^{n}} \int_{\mathbb{R}^{n}} f(y) \varphi\left(\frac{x-y}{\epsilon}\right) d y .
$$

\section{Então}

a) $f * \varphi_{\epsilon} \in C^{\infty}\left(\mathbb{R}^{n}\right)$.

b) $\operatorname{Se} f(x)=0$ q.t.p, fora de um conjunto fechado $A$, então supp $\left(f * \varphi_{\epsilon}\right) \subseteq A+\overline{B(0, \epsilon)}$.

c) Se $f$ é contínua e supp $(f)$ é compacto, então $f * \varphi_{\epsilon} \rightarrow f$ uniformemente quando $\epsilon \rightarrow 0$.

Demonstração. Para mostrar a), vamos mostrar primeiro que $f * \varphi_{\epsilon}$ é contínua. De fato, seja $\left\{x_{m}\right\}_{m \in \mathbb{N}}$ uma sequência em $\mathbb{R}^{n}$ tal que $x_{m} \rightarrow x$, como $\varphi$ é contínua, então existe uma constante $M>0$ tal que

$$
\begin{aligned}
\left|\left[\varphi_{\epsilon}\left(x_{m}-y\right)-\varphi_{\epsilon}(x-y)\right] f(y)\right| & =\left|\frac{1}{\epsilon^{n}}\left[\varphi\left(\frac{x_{m}-y}{\epsilon}\right)-\varphi\left(\frac{x-y}{\epsilon}\right)\right] f(y)\right| \\
& \leq \frac{2}{\epsilon^{n}} \sup _{z \in B(0,1)}|\varphi(z) \| f(y)| \\
& \leq M|f(y)| .
\end{aligned}
$$

Como $f \in L_{L o c}^{1}\left(\mathbb{R}^{n}\right)$ e $\varphi$ é contínua, vem do Teorema da convergência dominada que

$$
\begin{aligned}
\lim _{m \rightarrow \infty}\left[f * \varphi_{\epsilon}\left(x_{m}\right)-f * \varphi_{\epsilon}(x)\right] & =\lim _{m \rightarrow \infty} \int_{\mathbb{R}^{n}}\left[\varphi_{\epsilon}\left(x_{m}-y\right)-\varphi_{\epsilon}(x-y)\right] f(y) d y \\
& =\int_{\mathbb{R}^{n}} \lim _{m \rightarrow \infty}\left[\varphi_{\epsilon}\left(x_{m}-y\right)-\varphi_{\epsilon}(x-y)\right] f(y) d y \\
& =0
\end{aligned}
$$

o qual implica que

$$
\lim _{m \rightarrow \infty} f * \varphi_{\epsilon}\left(x_{m}\right)=f * \varphi_{\epsilon}(x)
$$

portanto $f * \varphi_{\epsilon}$ é contínua. 
Mostremos agora que $\partial\left(f * \varphi_{\epsilon}\right) / \partial x_{j}$ existem e é igual a $f * \partial \varphi_{\epsilon} / \partial x_{j}$, para todo $j \in \mathbb{N}$. De fato, Como $\partial \varphi_{\epsilon} / \partial x_{j}$ são contínuas para todo $j \in \mathbb{N}$, então existe uma constante $M>0$ tal que $\sup _{z \in B(0,1)}\left|d \varphi_{\epsilon}(z)\right| \leq M, \operatorname{logo}$

$$
\begin{aligned}
\frac{\partial}{\partial x_{j}}\left(f * \varphi_{\epsilon}(x)\right) & =\lim _{t \rightarrow 0} \frac{f * \varphi_{\epsilon}\left(x+t e_{j}\right)-f * \varphi_{\epsilon}(x)}{t} \\
& =\lim _{t \rightarrow 0} \frac{1}{t} \int_{\mathbb{R}^{n}}\left[\varphi_{\epsilon}\left(x-y+t e_{j}\right)-\varphi_{\epsilon}(x-y)\right] f(y) d y .
\end{aligned}
$$

Por outro lado, pela desigualdade do valor médio vem que

$\frac{1}{|t|}\left|\varphi_{\epsilon}\left(x-y+t e_{j}\right)-\varphi_{\epsilon}(x-y)\left\|f(y)\left|\leq \frac{M}{|t|}\right| x-y-\left(x-y+t e_{j}\right)\right\| f(y)\right|=M|f(y)|$,

aplicando o Teorema da convergência dominada obtemos

$$
\begin{aligned}
\frac{\partial}{\partial x_{j}}\left(f * \varphi_{\epsilon}(x)\right) & =\int_{\mathbb{R}^{n}} \lim _{t \rightarrow 0}\left[\frac{\varphi_{\epsilon}\left(x-y+t e_{j}\right)-\varphi_{\epsilon}(x-y)}{t}\right] f(y) d y \\
& =\int_{\mathbb{R}^{n}} \frac{\partial}{\partial x_{j}} \varphi_{\epsilon}(x-y) f(y) d y \\
& =f * \frac{\partial \varphi_{\epsilon}}{\partial x_{j}}
\end{aligned}
$$

Portanto $\partial\left(f * \varphi_{\epsilon}\right) / \partial x_{j}$ existe e, mais ainda, é contínua, ou seja, $f * \varphi_{\epsilon} \in C^{1}\left(\mathbb{R}^{n}\right)$.

No caso das derivadas superiores repetimos o argumento e obtemos $D^{\alpha}\left(f * \varphi_{\epsilon}\right)=f * D^{\alpha} \varphi_{\epsilon}$, para todo $\alpha \in \mathbb{Z}_{+}^{n}$.

Vamos mostrar agora $b$ ), por hipótese temos que

$$
f * \varphi_{\epsilon}(x)=\int_{\operatorname{supp}(\varphi)} f(x-\epsilon y) \varphi(y) d y
$$

Se $x_{0} \in \operatorname{int}(A)$, então existe $\delta>0$ tal que $B\left(x_{0}, \delta\right) \subset \operatorname{int}(A)$, ou seja, $f(x) \neq 0$, para todo $x \in B\left(x_{0}, \delta\right)$.

Por outro lado para todo $x \in B\left(x_{0}, \delta\right)$, existe $y_{x} \in \operatorname{supp}(\varphi) \subset \overline{B(0,1)}$ tal que

$$
x-\epsilon y_{x} \in B\left(x_{0}, \delta\right) \subset A,
$$

$\operatorname{logo} f * \varphi_{\epsilon}(x) \neq 0$, para todo $x \in B\left(x_{0}, \delta\right)$.

Agora para todo $x \in B\left(x_{0}, \delta\right) \subset A$ seja $a=x-\epsilon y_{x}$, temos que $x=a+\epsilon y_{x} \in A+\overline{B(0, \epsilon)}$. Portanto

$$
\operatorname{supp}\left(f * \varphi_{\epsilon}\right) \subseteq A+\overline{B(0, \epsilon)}
$$


Finalmente mostremos $c$ ). De fato, como $\operatorname{supp}(f)$ é compacto e pelo item $b$ ) temos $\operatorname{supp}\left(f * \varphi_{\epsilon}\right) \subseteq A+\overline{B(0, \epsilon)}, \operatorname{logo} \operatorname{supp}\left(f * \varphi_{\epsilon}\right)$ é compacto, pois basta tomar $A=\operatorname{supp}(f)$. Portanto obtemos que $f * \varphi_{\epsilon} \in C_{c}^{\infty}\left(\mathbb{R}^{n}\right)$.

Por outro lado, dado $\delta>0$, como $f$ é uniformemente contínua, existe $\epsilon_{0}>0$, tal que para todo $\epsilon \leq \epsilon_{0}$, usando o fato de que $\int \varphi(y) d y=1$, temos que

$$
\begin{aligned}
\left|f(x)-f * \varphi_{\epsilon}(x)\right| & =\left|\int_{\mathbb{R}^{n}} f(x) \varphi(y) d y-\int_{\mathbb{R}^{n}} f(x-\epsilon y) \varphi(y) d y\right| \\
& =\left|\int_{\mathbb{R}^{n}}[f(x)-f(x-\epsilon y)] \varphi(y) d y\right| \\
& \leq \sup _{y}|f(x)-f(x-\epsilon y)| \int_{\mathbb{R}^{n}} \varphi(y) d y \leq \delta,
\end{aligned}
$$

portanto,

$$
f * \varphi_{\epsilon} \longrightarrow f \quad \text { uniformemente. }
$$

Corolário 1.9. Se $f \in L^{1}\left(\mathbb{R}^{n}\right)$ e $f * \varphi_{\epsilon}$ é definido como em (1.1), então as seguintes condições são satisfeitas:

i) $\left\|f * \varphi_{\epsilon}\right\|_{L^{1}} \leq\|f\|_{L^{1}}$.

ii) $\left\|f * \varphi_{\epsilon}-f\right\|_{L^{1}} \rightarrow 0$ quando $\epsilon \rightarrow 0$.

Demonstração. Vamos mostrar o item $i$ ). De fato, pelo Teorema de Fubini temos que

$$
\begin{aligned}
\int_{\mathbb{R}^{n}}\left|f * \varphi_{\epsilon}(x)\right| d x & =\int_{\mathbb{R}^{n}}\left|\int_{\mathbb{R}^{n}} f(x-\epsilon y) \varphi(y) d y\right| d x \\
& \leq \int_{\mathbb{R}^{n}}\left[\int_{\mathbb{R}^{n}}|f(x-\epsilon y) \| \varphi(y)| d y\right] d x \\
& =\int_{\mathbb{R}^{n}} \varphi(y) \int_{\mathbb{R}^{n}}|f(x-\epsilon y)| d x d y .
\end{aligned}
$$

Por outro lado

$$
\int_{\mathbb{R}^{n}}|f(x-\epsilon y)| d x=\int_{\mathbb{R}^{n}}|f(x)| d x \quad \text { e } \quad \int_{\mathbb{R}^{n}} \varphi(y) d y=1 .
$$

Portanto obtemos que

$$
\left\|f * \varphi_{\epsilon}\right\|_{L^{1}}=\int_{\mathbb{R}^{n}}\left|f * \varphi_{\epsilon}(x)\right| d x \leq \int_{\mathbb{R}^{n}} \varphi(y) d y \int_{\mathbb{R}^{n}}|f(x)| d x=\|f\|_{L^{1}},
$$


ou seja,

$$
\left\|f * \varphi_{\epsilon}\right\|_{L^{1}} \leq\|f\|_{L^{1}}
$$

Para mostrar o item $i i)$ temos que dado $\delta>0$, usando o fato que $C_{c}^{\infty}\left(\mathbb{R}^{n}\right)$ é denso em $L^{1}\left(\mathbb{R}^{n}\right)$, existe $g \in C_{c}^{\infty}\left(\mathbb{R}^{n}\right)$, tal que

$$
\|f-g\|_{L^{1}}<\frac{\delta}{3}
$$

Logo usando (1.4) e o item $i$ ) temos

$$
\begin{aligned}
\left\|f-f * \varphi_{\epsilon}\right\|_{L^{1}} & =\left\|f-g+g-g * \varphi_{\epsilon}+g * \varphi_{\epsilon}-f * \varphi_{\epsilon}\right\|_{L^{1}} \\
& \leq\|f-g\|_{L^{1}}+\left\|g-g * \varphi_{\epsilon}\right\|_{L^{1}}+\left\|(g-f) * \varphi_{\epsilon}\right\|_{L^{1}} \\
& \leq\|f-g\|_{L^{1}}+\left\|g-g * \varphi_{\epsilon}\right\|_{L^{1}}+\|g-f\|_{L^{1}} \\
& <\frac{2 \delta}{3}+\left\|g-g * \varphi_{\epsilon}\right\|_{L^{1}} .
\end{aligned}
$$

Pelo item $b$ ) e $c$ ) do Teorema 1.8 temos que $g * \varphi_{\epsilon} \rightarrow g$ uniformemente quando $\epsilon \rightarrow 0$ e

$$
\operatorname{supp}\left(g * \varphi_{\epsilon}\right) \subseteq \operatorname{supp}(g)+\overline{B(0, \epsilon)} \subseteq \operatorname{supp}(g)+\overline{B(0,1)} \text {, para } 0<\epsilon<1
$$

Logo chamemos de $K=\operatorname{supp}(g)+\overline{B(0,1)}$, o qual é compacto , então temos

$$
\begin{aligned}
\left\|g-g * \varphi_{\epsilon}\right\|_{L^{1}} & =\int_{K}\left|g(x)-g * \varphi_{\epsilon}(x)\right| d x \\
& \leq \sup _{x \in K}\left|g(x)-g * \varphi_{\epsilon}(x)\right| \int_{K} d x \\
& <\frac{\delta}{3|K|}|K|=\frac{\delta}{3} .
\end{aligned}
$$

onde $|K|$ é a medida do conjunto $K$.

Portanto usando (1.5) e (1.6) temos que

$$
\left\|f-f * \varphi_{\epsilon}\right\|_{L^{1}}<\delta, \text { para } \epsilon>0 \text { suficientemente pequeno }
$$

Isto mostra $i i)$.

Corolário 1.10. Seja $K \subset \Omega \subseteq \mathbb{R}^{n}$, com $K$ compacto. Então existe $\psi \in C_{c}^{\infty}(\Omega)$ tal que $0 \leq \psi \leq 1$ e $\psi \equiv 1$ em uma vizinhança de $K$.

Demonstração. Seja $\delta=d\left(K, \Omega^{c}\right)=\inf \{|x-y| ; x \in K, y \notin \Omega\}$ como $K$ é compacto e $\Omega^{c}$ é fechado e disjuntos, temos que $\delta>0$. 
Sejam $\epsilon, \rho>0$ tal que $0<\epsilon<\rho<\epsilon+\rho<\delta$ e $\chi$ a função caraterística do conjunto $K_{\rho}=K+\overline{B(0, \rho)}$ (que é compacto), ou seja,

$$
\chi(x)= \begin{cases}1 & \text { se } x \in K_{\rho} \\ 0 & \text { se } x \notin K_{\rho}\end{cases}
$$

Agora consideremos $\psi(x)=\chi * \varphi_{\epsilon}(x)$, onde $\left\{\begin{array}{l}\varphi_{\epsilon}(x)=\frac{1}{\epsilon^{n}} \varphi\left(\frac{x}{\epsilon}\right), \int \varphi(x) d x=1 \\ \varphi \in C_{c}^{\infty}\left(\mathbb{R}^{n}\right), \operatorname{supp}(\varphi) \subset B(0,1)\end{array}\right.$

e $\varphi$ é dada como no Exemplo 1.5. Temos que

$$
\begin{aligned}
\operatorname{supp}(\psi) & \subset K_{\rho}+\overline{B(0, \epsilon)} \\
& \subseteq K+\overline{B(0, \rho)}+\overline{B(0, \epsilon)} \\
& =\underbrace{K+\overline{B(0, \rho+\epsilon)}}_{\text {compacto }} \subset \Omega
\end{aligned}
$$

vem que $\psi \in C_{c}^{\infty}(\Omega)$.

Por outro lado temos

$$
0 \leq \psi(x)=\int_{\Omega} \chi(x) \varphi_{\epsilon}(x-y) d y \leq \int_{\Omega} \varphi_{\epsilon}(x-y) d y \leq \int_{\mathbb{R}^{n}} \varphi_{\epsilon}(x-y) d y=\int_{\mathbb{R}^{n}} \varphi_{\epsilon}(y) d y=1
$$

assim obtemos que

$$
0 \leq \psi(x) \leq 1, \text { para todo } x \in \Omega
$$

Além disso, se $x \in \operatorname{supp}(\psi)$, segue que $d(x, K) \leq \rho+\epsilon$, daí $x=\tilde{x}+(\rho+\epsilon) y$, onde $\tilde{x} \in K$ e $y \in \overline{B(0,1)}$, então

$$
x-\epsilon y=\tilde{x}+\rho y \in K+\overline{B(0, \rho)}=K_{\rho} .
$$

Portanto se $x \in \operatorname{supp}(\psi)$, então

$$
\psi(x)=\int \chi(x-\epsilon y) \varphi(y) d y=\int_{|y| \leq 1} \overbrace{\chi(x-\epsilon y)}^{=1} \varphi(y) d y=\int_{|y| \leq 1} \varphi(y) d y=1 .
$$

Logo, $\psi \equiv 1$ em $\operatorname{supp}(\psi)$, que é uma vizinhança de $K$. Isto completa a demonstração do corolário. 


\subsection{As distribuições}

Nesta seção vamos definir o importante espaço das distribuições e estudar algumas de suas propriedades. Lembremos que um funcional linear é contínuo se e somente se ele for contínuo no ponto zero, a fim de usar este resultado é necessário saber o que significa convergência a zero em $C_{c}^{\infty}(\Omega)$, que é o que iremos fazer em seguida. Como antes $\Omega$ é um subconjunto aberto de $\mathbb{R}^{n}$.

Definição 1.11. Uma sequência de funções $\left\{\varphi_{j}\right\}_{j \in \mathbb{N}} \subset C_{c}^{\infty}(\Omega)$ converge a zero em $C_{c}^{\infty}(\Omega)$ se

i) Existe um compacto $K \subset \Omega$ tal que $\operatorname{supp}\left(\varphi_{j}\right) \subset K$, para todo $j=1,2, \ldots$

ii) Para todo inteiro positivo $m$, as derivadas até ordem $m$ das funções $\varphi_{j}$ convergem uniformemente a zero quando $j \rightarrow \infty$. Ou seja, $\partial^{\alpha} \varphi_{j} \rightarrow 0$ uniformemente quando $j \rightarrow \infty$, para todo $\alpha$ tal que $|\alpha| \leq m$, para todo $m=0,1, \ldots$

Nota: Diremos que uma sequência de funções $\left\{\varphi_{j}\right\}_{j \in \mathbb{N}} \subset C_{c}^{\infty}(\Omega)$ converge para a função $\varphi \in C_{c}^{\infty}(\Omega)$ quando $\left(\varphi_{j}-\varphi\right) \rightarrow 0 \mathrm{em} C_{c}^{\infty}(\Omega)$.

Observação 1.12. É possível dotar o espaço $C_{c}^{\infty}(\Omega)$ com uma topologia de forma que a convergência nessa topologia coincide com a dada pela definição acima (ver [15]). Vejamos que essa topologia não provem de uma métrica.

De fato, Seja $\left\{\varphi_{n}\right\}_{n \in \mathbb{N}}$ uma sequência de funções em $C_{c}^{\infty}(\Omega)$ e $\varphi \in C_{c}^{\infty}(\Omega)$, suponhamos por contradição que d é uma distância tal que $d\left(\varphi, \varphi_{n}\right) \rightarrow 0$ se e somente se $\left(\varphi_{n}-\varphi\right) \rightarrow 0$ em $C_{c}^{\infty}(\Omega)$. Considere $K_{m}$ uma sequência de compactos cuja união seja $\Omega$, logo pelo Corolário 1.10, pode-se escolher funções $\varphi_{m} \in C_{c}^{\infty}(\Omega)$ tal que $\varphi_{m} \equiv 1$ em uma vizinhança de $K_{m}$. Como para cada $m$ fixo temos que $\epsilon \varphi_{m} \rightarrow 0$ em $C_{c}^{\infty}(\Omega)$ quando $\epsilon \rightarrow 0$, logo por hipótese vem que existe $\epsilon_{m}>0$ tal que $d\left(\epsilon_{m} \varphi_{m}, 0\right)<1 / m$, agora fazendo variar $m$ temos que $d\left(\epsilon_{m} \varphi_{m}, 0\right) \rightarrow 0$, mas $\epsilon_{m} \varphi_{m} \nrightarrow 0$ em $C_{c}^{\infty}(\Omega)$, pois $K_{m} \subset \operatorname{supp}\left(\epsilon_{m} \varphi_{m}\right)$ o qual implica que $\bigcup_{m \in \mathbb{N}} \operatorname{supp}\left(\epsilon_{m} \varphi_{m}\right)=\Omega$, e isto contradiz o item i) da Definição 1.11.

A seguir vamos definir o espaço das distribuições.

Definição 1.13. Seja $\Omega \subset \mathbb{R}^{n}$ um subconjunto aberto, o espaço das distribuições é dado por

$$
\mathcal{D}^{\prime}(\Omega)=\left\{u: C_{c}^{\infty}(\Omega) \longrightarrow \mathbb{C} ; u \text { é linear e contínua }\right\} .
$$

Ou seja, $u \in \mathcal{D}^{\prime}(\Omega)$ se satisfaz a seguintes propriedades:

i) $u\left(\varphi_{1}+\alpha \varphi_{2}\right)=u\left(\varphi_{1}\right)+\alpha u\left(\varphi_{2}\right)$, para todo $\varphi_{1}, \varphi_{2} \in C_{c}^{\infty}(\Omega)$ e para todo $\alpha \in \mathbb{C}$. 
ii) $S e\left\{\varphi_{j}\right\}_{j \in \mathbb{N}}$ é uma sequência em $C_{c}^{\infty}(\Omega)$ tal que $\varphi_{j} \rightarrow 0$ quando $j \rightarrow \infty$ em $C_{c}^{\infty}(\Omega)$, então $u\left(\varphi_{j}\right) \rightarrow 0=u(0)$ em $\mathbb{C}$.

Notação: Geralmente denotaremos o valor da distribuição $u$ aplicada na função teste $\varphi$ por $\langle u, \varphi\rangle$ em lugar de $u(\varphi)$.

Exemplo 1.14. Considere $\Omega=\mathbb{R}^{n}$. Para $a \in \Omega$ defina $\delta_{a}: C_{c}^{\infty}\left(\mathbb{R}^{n}\right) \longrightarrow \mathbb{C}$ da seguinte maneira $\delta_{a}(\varphi)=\left\langle\delta_{a}, \varphi\right\rangle=\varphi(a), \log \mathrm{O}$

i) Sejam $\varphi_{1}, \varphi_{2} \in C_{c}^{\infty}\left(\mathbb{R}^{n}\right)$ e $\alpha \in \mathbb{C}$, temos que

$$
\begin{aligned}
\left\langle\delta_{a}, \varphi_{1}+\alpha \varphi_{2}\right\rangle & =\left(\varphi_{1}+\alpha \varphi_{2}\right)(a) \\
& =\varphi_{1}(a)+\alpha \varphi_{2}(a) \\
& =\left\langle\delta_{a}, \varphi_{1}\right\rangle+\alpha\left\langle\delta_{a}, \varphi_{2}\right\rangle
\end{aligned}
$$

ii) Seja $\left\{\varphi_{j}\right\}_{j \in \mathbb{N}} \subset C_{c}^{\infty}\left(\mathbb{R}^{n}\right)$ uma sequência de funções tal que $\varphi_{j} \rightarrow 0$ em $C_{c}^{\infty}\left(\mathbb{R}^{n}\right)$, vem que $\sup _{K}\left|\varphi_{j}\right| \rightarrow 0$ quando $j \rightarrow \infty$, em particular $\varphi_{j}(a) \rightarrow 0$ quando $j \rightarrow \infty$ em $\mathbb{C}$, ou seja $\left\langle\delta_{a}, \varphi_{j}\right\rangle \rightarrow 0$ quando $j \rightarrow \infty$.

Portanto de $i$ ) e $i i)$ segue que $\delta_{a}$ é uma distribuição, chamada de Delta de Dirac centrada no ponto $a$.

Exemplo 1.15. Seja $\Omega=\mathbb{R}$ e defina $T: C_{c}^{\infty}(\mathbb{R}) \longrightarrow \mathbb{C}$ da seguinte forma

$$
T(\varphi)=\langle T, \varphi\rangle=\int_{-\infty}^{\infty}|t| \varphi^{\prime}(t) d t, \quad \text { para todo } \varphi \in C_{c}^{\infty}(\mathbb{R})
$$

Mostremos que T é uma distribuição. De fato,

i) Sejam $\varphi_{1}, \varphi_{2} \in C_{c}^{\infty}(\mathbb{R})$ e $\alpha \in \mathbb{C}, \log 0$

$$
\begin{aligned}
\left\langle T, \varphi_{1}+\alpha \varphi_{2}\right\rangle & =\int_{-\infty}^{\infty}|t|\left(\varphi_{1}+\alpha \varphi_{2}\right)^{\prime}(t) d t=\int_{-\infty}^{\infty}\left[|t| \varphi_{1}^{\prime}(t)+|t| \alpha \varphi_{2}^{\prime}(t)\right] d t \\
& =\int_{-\infty}^{\infty}|t| \varphi_{1}^{\prime}(t) d t+\alpha \int_{-\infty}^{\infty}|t| \varphi_{2}^{\prime}(t) d t=\left\langle T, \varphi_{1}\right\rangle+\alpha\left\langle T, \varphi_{2}\right\rangle .
\end{aligned}
$$

ii) Seja $\left\{\varphi_{j}\right\}_{j \in \mathbb{N}} \subset C_{c}^{\infty}(\mathbb{R})$ uma sequência de funções tal que $\varphi_{j} \rightarrow 0$, quando $j \rightarrow \infty$ em $C_{c}^{\infty}(\mathbb{R})$, então existe $a>0$ tal que $\operatorname{supp}\left(\varphi_{j}\right) \subset[-a, a]$ para todo $j \in \mathbb{N}$, além 
disso, $\varphi_{j}^{\prime}(t) \rightarrow 0$ uniformemente em $\mathbb{C}$, logo

$$
\begin{aligned}
\left|\left\langle T, \varphi_{j}\right\rangle\right| & \leq \int_{-\infty}^{\infty}|t|\left|\varphi_{j}^{\prime}(t)\right| d t=\int_{-a}^{a}|t|\left|\varphi_{j}^{\prime}(t)\right| d t \\
& \leq \sup _{t \in[-a, a]}\left|\varphi_{j}^{\prime}(t)\right| \int_{-a}^{a}|t| d t=a^{2} \sup _{t \in[-a, a]}\left|\varphi_{j}^{\prime}(t)\right| .
\end{aligned}
$$

Segue-se que $\left|\left\langle T, \varphi_{j}\right\rangle\right| \leq a^{2} \sup _{t \in[-a, a]}\left|\varphi_{j}^{\prime}(t)\right|$ e como $\sup _{t \in[-a, a]}\left|\varphi_{j}^{\prime}(t)\right| \rightarrow 0$ em $\mathbb{C}$, quando $j \rightarrow \infty$, então

$$
\left\langle T, \varphi_{j}\right\rangle \rightarrow 0 \text { em } \mathbb{C} \text {, quando } j \rightarrow \infty
$$

Logo de $i$ ) e ii) temos que $T$ é uma distribuição.

Exemplo 1.16. Seja $f \in L_{L o c}^{1}(\Omega)$, onde $\Omega \subseteq \mathbb{R}^{n}$ é subconjunto aberto. Defina $T_{f}: C_{c}^{\infty}(\Omega) \longrightarrow \mathbb{C}$ da seguinte forma

$$
\left\langle T_{f}, \varphi\right\rangle=\int_{\Omega} f(x) \varphi(x) d x \quad, \quad \varphi \in C_{c}^{\infty}(\Omega)
$$

temos que $T_{f}$ é bem definida, além disso,

i) Sejam $\varphi_{1}, \varphi_{2} \in C_{c}^{\infty}(\Omega)$ e $\alpha \in \mathbb{C}$, temos que

$$
\begin{aligned}
\left\langle T_{f}, \varphi_{1}+\alpha \varphi_{2}\right\rangle & =\int_{\Omega} f(x)\left(\varphi_{1}+\alpha \varphi_{2}\right)(x) d x \\
& =\int_{\Omega} f(x) \varphi_{1}(x) d x+\alpha \int_{\Omega} f(x) \varphi_{2}(x) d x \\
& =\left\langle T_{f}, \varphi_{1}\right\rangle+\alpha\left\langle T_{f}, \varphi_{2}\right\rangle
\end{aligned}
$$

ii) Seja $\left\{\varphi_{j}\right\}_{j \in \mathbb{N}} \subset C_{c}^{\infty}(\Omega)$ uma sequência de funções tal que $\varphi_{j} \rightarrow 0$ em $C_{c}^{\infty}(\Omega)$, quando $j \rightarrow \infty$. Então $\sup _{K}\left|\varphi_{j}(x)\right| \rightarrow 0$ em $\mathbb{C}$. Por outro lado, temos

$$
\left|\left\langle T_{f}, \varphi_{j}\right\rangle\right| \leq \int_{\operatorname{supp}\left(\varphi_{j}\right)}|f(x)|\left|\varphi_{j}(x)\right| d x \leq \sup _{K}\left|\varphi_{j}(x)\right| \int_{K}|f(x)| d x
$$

o qual implica que

$$
\left\langle T_{f}, \varphi_{j}\right\rangle \rightarrow 0 \text { em } \mathbb{C} \text { quando } j \rightarrow \infty \text {. }
$$

pois $\sup _{K}\left|\varphi_{j}(x)\right| \rightarrow 0$ em $\mathbb{C}$ e $\int_{K}|f(x)| d x$ é finita.

Logo de $i$ ) e $i i)$ segue que $T_{f}$ é uma distribuição. 
Observação 1.17. Suponha que $f, g \in L_{\text {Loc }}^{1}(\Omega)$ tais que $T_{f}=T_{g}$ no sentido das distribuições, isto é $\left\langle T_{f}, \varphi\right\rangle=\left\langle T_{g}, \varphi\right\rangle$ para toda $\varphi \in C_{c}^{\infty}(\Omega)$. Mostremos que $f=g$ q.t.p.

De fato, seja $K \subset \Omega$ um subconjunto compacto tal que $\alpha \in C_{c}^{\infty}(\Omega)$ e $\alpha \equiv 1$ em $K$, seja $h=f-g$ e considere a seguinte função

$$
H=\left\{\begin{array}{cc}
\alpha h & \text { se } x \in \Omega \\
0 & \text { se } x \in \Omega^{c}
\end{array}\right.
$$

Observe que $H \in L_{\text {Loc }}^{1}\left(\mathbb{R}^{n}\right)$, além disso, temos

$$
\begin{aligned}
H * \varphi_{\epsilon}(x) & =\epsilon^{-n} \int_{\mathbb{R}^{n}} H(y) \varphi\left(\frac{x-y}{\epsilon}\right) d y=\epsilon^{-n} \int_{\mathbb{R}^{n}}(\alpha h)(y) \varphi\left(\frac{x-y}{\epsilon}\right) d y \\
& =\epsilon^{-n} \int_{\Omega} \alpha(y) f(y) \varphi\left(\frac{x-y}{\epsilon}\right) d y-\epsilon^{-n} \int_{\Omega} \alpha(y) g(y) \varphi\left(\frac{x-y}{\epsilon}\right) d y \\
& =\left\langle T_{f}, \beta_{\epsilon}^{x}\right\rangle-\left\langle T_{g}, \beta_{\epsilon}^{x}\right\rangle=0 \quad, \quad \text { onde } \beta_{\epsilon}^{x}(y)=\alpha(y) \frac{1}{\epsilon^{n}} \varphi\left(\frac{x-y}{\epsilon}\right) \in C_{c}^{\infty}(\Omega)
\end{aligned}
$$

portanto $H * \varphi_{\epsilon}(x)=0$ para todo $x \in \mathbb{R}^{n}$ e $\epsilon>0$.

Por outro lado sabemos que $H * \varphi_{\epsilon} \longrightarrow H$ em $L^{1}$, ou seja $H=0$ q.t.p ou $\alpha h(x)=0$ q.t.p x. Logo em um compacto $K$ temos que $h=0$ q.t.p $\operatorname{logo} f=g$ q.t.p em $K$, fazendo $\Omega=\bigcup K_{n}$ onde $K_{n}$ é compacto teremos que $f=g$ q.t.p em $\Omega$.

Pela observação acima, podemos imediatamente constatar que se $f \in L_{L o c}^{1}(\Omega)$ e $\left\langle T_{f}, \varphi\right\rangle=0$ para toda $\varphi \in C_{c}^{\infty}(\Omega)$, então $f=0$ q.t.p. A distribuição $T_{f}$ sera denotada como $f$, e escrevemos simplesmente $\langle f, \varphi\rangle=\int_{\Omega} f(x) \varphi(x) d x$, obtendo assim uma identificação entre qualquer função localmente integrável $f$ com o funcional $T_{f}$ definido no Exemplo 1.16. Esta identificação permite considerar o seguinte $C^{k}(\Omega) \subset L_{L o c}^{p}(\Omega) \subset$ $L_{\text {Loc }}^{1}(\Omega) \subset \mathcal{D}^{\prime}(\Omega)$, onde $1 \leq k \leq \infty$ e $1 \leq p \leq \infty$.

\subsubsection{Operações com distribuições}

A soma e o produto por um escalar de distribuições definem-se da maneira óbvia. Se $u_{1}, u_{2} \in \mathcal{D}^{\prime}(\Omega)$ e $\alpha \in \mathbb{C}$, então para toda $\varphi \in C_{c}^{\infty}(\Omega)$ temos

$$
\begin{aligned}
\left\langle u_{1}+u_{2}, \varphi\right\rangle & =\left\langle u_{1}, \varphi\right\rangle+\left\langle u_{2}, \varphi\right\rangle \\
\left\langle\alpha u_{1}, \varphi\right\rangle & =\alpha\left\langle u_{1}, \varphi\right\rangle .
\end{aligned}
$$

Mostrar que $u_{1}+u_{2}$ e $\alpha u_{1}$ são distribuições não apresenta grandes dificuldades. 
Para definirmos outros tipos de operações que envolvem distribuições precisamos da noção de operador linear contínuo sobre $C_{c}^{\infty}(\Omega)$ que definimos a seguir.

Definição 1.18. $L: C_{c}^{\infty}(\Omega) \longrightarrow C_{c}^{\infty}(\Omega)$ é um operador linear contínuo se é linear $e$ satisfaz o seguinte: dada uma sequência $\left\{\varphi_{j}\right\}_{j \in \mathbb{N}} \subset C_{c}^{\infty}(\Omega)$ tal que $\varphi_{j} \rightarrow 0$ em $C_{c}^{\infty}(\Omega)$, então $L \varphi_{j} \rightarrow 0$ em $C_{c}^{\infty}(\Omega)$.

Exemplo 1.19. $L=\frac{\partial}{\partial x_{j}}: C_{c}^{\infty}(\Omega) \longrightarrow C_{c}^{\infty}(\Omega)$ definido por

$$
\frac{\partial}{\partial x_{j}}(\varphi)=\frac{\partial \varphi}{\partial x_{j}} \quad, \quad \varphi \in C_{c}^{\infty}(\Omega)
$$

é um operador linear contínuo.

Exemplo 1.20. Seja $f \in C^{\infty}(\Omega)$ e defina $L_{f}: C_{c}^{\infty}(\Omega) \longrightarrow C_{c}^{\infty}(\Omega)$ da seguinte forma

$$
L_{f}(\varphi)=f \varphi \quad, \quad \varphi \in C_{c}^{\infty}(\Omega)
$$

então $L_{f}$ é um operador linear contínuo.

Definição 1.21. (Transposto Formal)

Sejam $L, L^{t}: C_{c}^{\infty}(\Omega) \longrightarrow C_{c}^{\infty}(\Omega)$ operadores lineares continuos. Dizemos que $L^{t}$ é o transposto formal de $L$ se

$$
\int_{\Omega}(L \varphi) \psi d x=\int_{\Omega} \varphi\left(L^{t} \psi\right) d x \quad, \quad \text { para todo } \varphi, \psi \in C_{c}^{\infty}(\Omega)
$$

Exemplo 1.22. Seja $L_{f}(\varphi)=f \varphi$, com $f \in C^{\infty}(\Omega)$ e $\varphi \in C_{c}^{\infty}(\Omega)$, então temos

$$
\int_{\Omega} L_{f}(\varphi) \psi d x=\int_{\Omega}(f \varphi) \psi d x=\int_{\Omega} \varphi(f \psi) d x=\int_{\Omega} \varphi L_{f}(\psi) d x
$$

portanto $L_{f}^{t}=L_{f}$.

Exemplo 1.23. Considere $L=\frac{\partial}{\partial x_{j}}$, vamos obter o seu transposto formal. Para isto $\operatorname{sejam} \varphi, \psi \in C_{c}^{\infty}(\Omega) \operatorname{logo}$

$$
\begin{aligned}
\int_{\Omega} L(\varphi) \psi d x & =\int_{\Omega} \frac{\partial \varphi}{\partial x_{j}} \psi=\left.\varphi \psi\right|_{\partial(\operatorname{supp}(\varphi))}-\int_{\Omega} \varphi \frac{\partial \psi}{\partial x_{j}} d x \\
& =\int_{\Omega} \varphi\left(-\frac{\partial \psi}{\partial x_{j}}\right) d x=\int_{\Omega} \varphi L^{t}(\psi) d x
\end{aligned}
$$


portanto obtemos que $L^{t}=-\frac{\partial}{\partial x_{j}}$.

Exemplo 1.24. Seja $L=a \frac{\partial}{\partial x_{j}}$, com $a \in C^{\infty}(\Omega)$. Se $\varphi, \psi \in C_{c}^{\infty}(\Omega)$ temos que

$$
\begin{aligned}
\int_{\Omega} L(\varphi) \psi d x & =\int_{\Omega} a \frac{\partial \varphi}{\partial x_{j}} \psi d x=\int_{\Omega} \frac{\partial \varphi}{\partial x_{j}} a \psi d x \\
& =-\int_{\Omega} \varphi \frac{\partial}{\partial x_{j}}(a \psi) d x=\int_{\Omega} \varphi\left(-\frac{\partial a}{\partial x_{j}}-a \frac{\partial}{\partial x_{j}}\right) \psi d x
\end{aligned}
$$

portanto segue que $L^{t}=-\frac{\partial a}{\partial x_{j}}-a \frac{\partial}{\partial x_{j}}=-\frac{\partial a}{\partial x_{j}}-L$.

Exemplo 1.25. Considere o operador $L=\frac{\partial^{2}}{\partial x_{j}^{2}}$. Se $\varphi, \psi \in C_{c}^{\infty}(\Omega)$, temos que

$$
\int_{\Omega} L(\varphi) \psi d x=\int_{\Omega} \frac{\partial^{2} \varphi}{\partial x_{j}^{2}} \psi d x=-\int_{\Omega} \frac{\partial \varphi}{\partial x_{j}} \frac{\partial \psi}{\partial x_{j}} d x=\int_{\Omega} \varphi \frac{\partial^{2} \psi}{\partial x_{j}^{2}} d x
$$

portanto $L^{t}=L$.

Observação 1.26. Notemos que $\varphi, L \varphi, \psi, L^{t} \psi \in C_{c}^{\infty}(\Omega) \subseteq L_{L o c}^{1}(\Omega) \subseteq \mathcal{D}^{\prime}(\Omega)$ e portanto a equação (1.7) pode também ser escrita da forma

$$
\langle L \varphi, \psi\rangle=\left\langle\varphi, L^{t} \psi\right\rangle
$$

Podemos então estender $L$ para um operador $\tilde{L}$ em $\mathcal{D}^{\prime}(\Omega)$, ou seja, $\tilde{L}: \mathcal{D}^{\prime}(\Omega) \longrightarrow \mathcal{D}^{\prime}(\Omega)$ definido por

$$
\langle\tilde{L} u, \psi\rangle=\left\langle u, L^{t} \psi\right\rangle \quad, \quad u \in \mathcal{D}^{\prime}(\Omega), \psi \in C_{c}^{\infty}(\Omega) .
$$

Exemplo 1.27. (produto por uma função $C^{\infty}$ )

Seja $f \in C^{\infty}(\Omega)$ e $u \in \mathcal{D}^{\prime}(\Omega)$, pelo Exemplo 1.22 a "operação multiplicação por $f$ " fica definida pela seguinte expressão

$$
\langle f u, \varphi\rangle=\langle u, f \varphi\rangle, \quad \text { para toda } \varphi \in C_{c}^{\infty}(\Omega)
$$

Consideremos, por exemplo, a distribuição Delta de Dirac centrada em $a \in \mathbb{R}^{n}, \delta_{a}$ e $f$ qualquer função em $C^{\infty}\left(\mathbb{R}^{n}\right)$ que esteja definida em $a$, então para toda $\varphi \in C_{c}^{\infty}\left(\mathbb{R}^{n}\right)$ temos

$$
\left\langle f \delta_{a}, \varphi\right\rangle=\left\langle\delta_{a}, f \varphi\right\rangle=f(a) \varphi(a)=\left\langle f(a) \delta_{a}, \varphi\right\rangle
$$

Ou seja, obtemos que $f \delta_{a}=f(a) \delta_{a}$. 
Exemplo 1.28. (derivação)

seja $u \in \mathcal{D}^{\prime}(\Omega)$, então pelo Exemplo 1.23, podemos definir a operação "derivação" da seguinte forma

$$
\left\langle\frac{\partial u}{\partial x_{j}}, \varphi\right\rangle=-\left\langle u, \frac{\partial \varphi}{\partial x_{j}}\right\rangle, \quad \text { para toda } \varphi \in C_{c}^{\infty}(\Omega) .
$$

Por aplicação repetida da expressão acima e indução em $|\alpha|$ vemos que, dados um multiíndice $\alpha \in \mathbb{Z}_{+}^{n}$ e $u \in \mathcal{D}^{\prime}(\Omega)$, então

$$
\left\langle\partial^{\alpha} u, \varphi\right\rangle=(-1)^{|\alpha|}\left\langle u, \partial^{\alpha} \varphi\right\rangle, \text { para toda } \varphi \in C_{c}^{\infty}(\Omega)
$$

Exemplo 1.29. Considere $L=a \frac{\partial}{\partial x_{j}} \operatorname{com} a \in C^{\infty}(\Omega)$. Se $u \in \mathcal{D}^{\prime}(\Omega)$, temos que

$$
\langle L u, \varphi\rangle=\left\langle u,\left(-\frac{\partial a}{\partial x_{j}}\right) \varphi\right\rangle-\left\langle u, a \frac{\partial \varphi}{\partial x_{j}}\right\rangle, \quad \text { para toda } \varphi \in C_{c}^{\infty}(\Omega) .
$$

Exemplo 1.30. (Mudança de variáveis)

Seja $\Phi: \Omega \longrightarrow \Omega$ um difeomorfismo, isto é $\Phi, \Phi^{-1}$ são bijeções de classe $C^{\infty}$ sobre $\Omega$.

Definimos $L: C_{c}^{\infty}(\Omega) \longrightarrow C_{c}^{\infty}(\Omega)$ da seguinte forma

$$
L(\varphi)=\varphi \circ \Phi, \quad \text { para toda } \varphi \in C_{c}^{\infty}(\Omega) .
$$

Observe que $\operatorname{supp}(\varphi \circ \Phi)=\Phi^{-1}(\operatorname{supp}(\varphi))$ e, portanto, $L(\varphi) \in C_{c}^{\infty}(\Omega)$. Logo

$$
\begin{aligned}
\int_{\Omega} L(\varphi) \psi d x & =\int_{\Omega} \varphi(\Phi(x)) \psi(x) d x=\int_{\Omega} \varphi(\Phi(x)) \psi\left(\Phi^{-1} \circ \Phi(x)\right) d x \\
& =\int_{\Omega} \varphi(y) \psi\left(\Phi^{-1}(y)\right)\left|J \Phi^{-1}(y)\right| d y \quad \text { onde } y=\Phi(x)
\end{aligned}
$$

portanto $L^{t}(\psi)=\left|J \Phi^{-1}(y)\right| \psi \circ \Phi^{-1}$.

Logo se $u \in \mathcal{D}^{\prime}(\Omega)$ definimos então

$$
\langle u \circ \Phi, \psi\rangle=\left\langle u, L^{t}(\psi)\right\rangle=\left\langle u, \psi \circ \Phi^{-1}\left|J \Phi^{-1}\right|\right\rangle .
$$

É fácil ver que a regra de Leibniz para a derivada do produto de duas funções se mantém quando um dos fatores é uma distribuição, ou seja se $u \in \mathcal{D}^{\prime}(\Omega)$ e $f \in C^{\infty}(\Omega)$ se satisfaz que

$$
\frac{\partial(f u)}{\partial x_{j}}=f \frac{\partial u}{\partial x_{j}}+u \frac{\partial f}{\partial x_{j}} \text { para } j=1, \ldots, n .
$$

Para a demonstração de este fato consultar [6] pag.22. 
A seguir vamos provar um resultado que é muito importante na resolução de EDP's, pois permite procurar soluções de equações diferenciais em um espaço com muito mais exemplares que o usual.

Teorema 1.31. Se u e $f$ são funções contínuas definidas em $\Omega$ e $\frac{\partial u}{\partial x_{j}}=f$ no sentido das distribuições. Então u é diferenciável em relação a $x_{j}$ e $\frac{\partial u}{\partial x_{j}}=f$ no sentido clássico.

Demonstração. Suponha primeiro que $\operatorname{supp}(u)$ é compacto, então pelo Teorema 1.8 sabemos que $u * \varphi_{\epsilon} \rightarrow u$ uniformemente. Por outro lado temos

$$
\begin{aligned}
\frac{\partial}{\partial x_{j}}\left(u * \varphi_{\epsilon}\right)(x) & =\epsilon^{-n} \int_{\mathbb{R}^{n}} u(y) \frac{\partial}{\partial x_{j}} \varphi\left(\frac{x-y}{\epsilon}\right) d y \\
& =-\epsilon^{-n} \int_{\mathbb{R}^{n}} u(y) \frac{\partial}{\partial y_{j}} \varphi\left(\frac{x-y}{\epsilon}\right) d y \\
& =\left\langle\frac{\partial u}{\partial y_{j}}, \varphi_{\epsilon}(x-\cdot)\right\rangle \\
& =\left\langle f, \varphi_{\epsilon}(x-\cdot)\right\rangle=f * \varphi_{\epsilon}(x) \rightarrow f \text { uniformemente }
\end{aligned}
$$

ou seja, temos o seguinte

$$
u * \varphi_{\epsilon} \rightarrow u \text { e } \frac{\partial}{\partial x_{j}}\left(u * \varphi_{\epsilon}\right) \rightarrow f \text { uniformemente quando } \epsilon \rightarrow 0 \text {. }
$$

Portanto vem que $\frac{\partial u}{\partial x_{j}}=f$ no sentido clássico.

Para mostrar o caso geral, seja $x_{0} \in \Omega$ e pelo Corolário 1.10 temos que existe $\psi \in$ $C_{c}^{\infty}(\Omega)$, tal que $\psi \equiv 1$ numa vizinhança de $x_{0}$. Logo $\psi u$ é contínua, tem suporte compacto e por (1.8) vem que

$$
\frac{\partial}{\partial x_{j}}(\psi u)=\psi \frac{\partial u}{\partial x_{j}}+u \frac{\partial \psi}{\partial x_{j}}=\psi f+u \frac{\partial \psi}{\partial x_{j}} \operatorname{em} \mathcal{D}^{\prime}(\Omega)
$$

Além disso, $\psi f+u \frac{\partial \psi}{\partial x_{j}}$ é contínua, então pelo que foi provado antes temos que

$$
\frac{\partial}{\partial x_{j}}(\psi u)=\psi f+u \frac{\partial \psi}{\partial x_{j}} \text { no sentido clássico, }
$$

mas como $\psi \equiv 1$ numa vizinhança de $x_{0}$ segue que

$$
\frac{\partial u}{\partial x_{j}}\left(x_{0}\right)=f\left(x_{0}\right)
$$

e como $x_{0}$ foi escolhido de forma abitrária em $\Omega$, o teorema está provado. 


\subsubsection{Igualdade e suporte de distribuições}

Bem como na teoria de funções, na teoria das distribuições também pode-se definir a igualdade entre duas distribuições e o suporte de uma distribuição, mas antes de dar essas definições vamos enunciar e mostrar o seguinte resultado importante.

Teorema 1.32. Seja $K$ um subconjunto compacto de $\mathbb{R}^{n}$ e consideremos abertos $V_{1}, \ldots, V_{l}$ tais que $K \subseteq \bigcup_{j=1}^{l} V_{j}$. Então existem funções $\phi_{j} \in C_{c}^{\infty}\left(V_{j}\right)$ tais que satisfazem as seguintes condições:

i) $\sum_{j=1}^{l} \phi_{j}(x) \leq 1$.

ii) $\sum_{j=1}^{l} \phi_{j}(x)=1$ numa vizinhança de $K$.

iii) $0 \leq \phi_{j} \leq 1$ em $\mathbb{R}^{n}$ para $j=1,2, \ldots, l$.

Demonstração. Para cada $j=1,2, \ldots, l$ escolhemos compactos $K_{j} \subseteq V_{j}$ tal que $K \subset$ $\bigcup_{j=1}^{l} K_{j}$. Em seguida construímos funções $\psi_{j} \in C_{c}^{\infty}\left(V_{j}\right), j=1,2, \ldots, l$ tais que $0 \leq \psi_{j} \leq 1$ e $\psi_{j} \equiv 1$ numa vizinhança de $K_{j}$. Agora defina as seguintes funções

$$
\begin{aligned}
\phi_{1} & =\psi_{1} \\
\phi_{2} & =\psi_{2}\left(1-\psi_{1}\right) \\
\phi_{3} & =\psi_{3}\left(1-\psi_{1}\right)\left(1-\psi_{2}\right) \\
\vdots & \vdots \\
\phi_{l} & =\psi_{l}\left(1-\psi_{1}\right) \cdots\left(1-\psi_{l-1}\right) .
\end{aligned}
$$

Assim, usando indução sobre $l$, obtemos a seguinte igualdade

$$
\sum_{j=1}^{l} \phi_{j}=1-\left(1-\psi_{1}\right) \cdots\left(1-\psi_{l}\right)
$$

Para verificar $i$ ) temos que para todo $j=1, \ldots, l$ se satisfaz que $0 \leq \psi_{j} \leq 1$, o qual implica que $0 \leq\left(1-\psi_{j}\right) \leq 1$, para todo $j=1, \ldots, l$, logo obtemos

$$
0 \leq\left(1-\psi_{1}\right)\left(1-\psi_{2}\right) \cdots\left(1-\psi_{l}\right) \leq 1
$$

daí segue que

$$
0 \leq \sum_{j=1}^{l} \phi_{j}(x) \leq 1 \quad, \quad \text { para todo } x \in \mathbb{R}^{n} .
$$

Para verificar $i i)$ seja $U_{j}$ a vizinhança de $K_{j}$ tal que $\psi_{j} \equiv 1$ em $U_{j}$ onde $j=1, \ldots, l$, $\operatorname{assim} K \subset \bigcup_{j=1}^{l} K_{j} \subset \bigcup_{j=1}^{l} U_{j}$, ou seja, $\bigcup_{j=1}^{l} U_{j}$ é uma vizinhança de $K$, além disso, se 
$x \in \bigcup_{j=1}^{l} U_{j}$, então $x \in U_{j}$ para algum $j$, o qual implica que $\psi_{j}(x)=1$. Portanto obtemos que

$$
\sum_{j=1}^{l} \phi_{j}(x)=1-\left(1-\psi_{1}(x)\right) \cdots\left(1-\psi_{j}(x)\right) \cdots\left(1-\psi_{l}(x)\right)=1 .
$$

Finalmente verifiquemos $i i i)$, sabemos que para todo $j=1, \ldots, l$ se satisfaz que $0 \leq \psi_{j} \leq 1$ e $0 \leq\left(1-\psi_{j}\right) \leq 1$ em $\mathbb{R}^{n}$, daí segue que

$$
0 \leq \psi_{j}\left(1-\psi_{1}\right)\left(1-\psi_{2}\right) \cdots\left(1-\psi_{j-1}\right) \leq 1 \quad \text { em } \mathbb{R}^{n}
$$

ou seja,

$$
0 \leq \phi_{j} \leq 1 \quad \text { em } \mathbb{R}^{n}, \text { para } j=1, \ldots, l
$$

Definição 1.33. Sejam $u_{1}, u_{2} \in \mathcal{D}^{\prime}(\Omega)$, dizemos que $u_{1}$ é igual a $u_{2}$ em um aberto $U \subset \Omega$ e escrevemos $u_{1}=u_{2}$, quando $\left\langle u_{1}, \varphi\right\rangle=\left\langle u_{2}, \varphi\right\rangle$, para todo $\varphi \in C_{c}^{\infty}(U)$.

Teorema 1.34. Sejam $u_{1}, u_{2} \in \mathcal{D}^{\prime}(\Omega)$ tais que todo ponto de $\Omega$ tem uma vizinhança onde $u_{1}=u_{2}$. Então $u_{1}=u_{2}$ em $\Omega$.

Demonstração. Seja $\varphi \in C_{c}^{\infty}(\Omega)$ e $K=\operatorname{supp}(\varphi) \subset \Omega$. Escrevemos $\Omega=\bigcup_{x \in \Omega} U_{x}$, onde $U_{x}$ é tal que $u_{1}=u_{2}$ em $U_{x}$, mas $K \subset \Omega=\bigcup_{x \in \Omega} U_{x}$ e como $K$ é compacto obtemos $x_{1}, \ldots, x_{m} \in \Omega$ tais que

$$
K \subset \bigcup_{j=1}^{m} U_{x_{j}} \quad \text { e } \quad u_{1}=u_{2} \text { em } U_{x_{j}}, j=1, \ldots, m
$$

Agora pelo Teorema 1.32, existem funções $\phi_{j} \in C_{c}^{\infty}\left(U_{x_{j}}\right), j=1, \ldots, m$ tais que

$$
\sum_{j=1}^{m} \phi_{j}(x)=1 \quad \text { em uma vizinhança de } K
$$

$\log 0$

$$
\varphi(x)=\sum_{j=1}^{m} \phi_{j}(x) \varphi(x), \quad \text { para todo } x \in K
$$

Segue-se que

$$
\left\langle u_{1}, \varphi\right\rangle=\left\langle u_{1}, \sum_{j=1}^{m} \phi_{j} \varphi\right\rangle=\sum_{j=1}^{m}\left\langle u_{1}, \phi_{j} \varphi\right\rangle=\sum_{j=1}^{m}\left\langle u_{2}, \phi_{j} \varphi\right\rangle=\left\langle u_{2}, \sum_{j=1}^{m} \phi_{j} \varphi\right\rangle=\left\langle u_{2}, \varphi\right\rangle
$$


onde a terceira igualdade se satisfaz porque $\phi_{j} \varphi \in C_{c}^{\infty}\left(U_{x_{j}}\right)$. Portanto, obtemos que

$$
u_{1}=u_{2} \text { em } \Omega
$$

Definição 1.35. Se $u \in \mathcal{D}^{\prime}(\Omega)$ definimos o suporte de $u$, supp $(u)$, como a intersecção de todos os fechados de $\Omega$ fora dos quais u é nulo. Isto é, se $\Lambda$ é um conjunto de indices e para cada $\alpha \in \Lambda, F_{\alpha}$ é um subconjunto fechado de $\Omega$, então

$$
\operatorname{supp}(u)=\bigcap_{\alpha \in \Lambda} F_{\alpha} \quad \text { tal que } u=0 \text { em } F_{\alpha}^{c}
$$

Equivalentemente, supp $(u)$ é o complementar do maior aberto $U$ de $\Omega$ tal que $u=0$ em $U$.

Observação 1.36. Decorre diretamente da Definição 1.35, que para mostrar que um determinado ponto $a \in \Omega$ pertence ao $\operatorname{supp}(u)$ é suficiente mostrar que para toda bola aberta $B(a, r) \subset \Omega$, existe uma função $\varphi \in C_{c}^{\infty}(B(a, r))$ tal que $\langle u, \varphi\rangle \neq 0$.

De fato, seja $a \in \operatorname{supp}(u)$ e suponhamos que exista uma bola de raio $r>0$ centrada em a tal que para toda função $\varphi \in C_{c}^{\infty}(B(a, r))$ tenhamos $\langle u, \varphi\rangle=0$. Como $B(a, r)$ é aberto, temos que $F=\Omega \backslash B(a, r)$ é um conjunto fechado. Mas supp $(u)$ é a interseção de todos os fechados de $\Omega$ fora dos quais $u=0$ e a $\notin F$, logo a não pertence à interseção destes fechados; portanto c $\notin \operatorname{supp}(u)$, o que é uma contradição.

Definição 1.37. Se $\Omega$ é um subconjunto aberto de $\mathbb{R}^{n}$, denotamos com $\mathcal{E}^{\prime}(\Omega)$ o subespaço de $\mathcal{D}^{\prime}(\Omega)$ das distribuições com suporte compacto. Ou seja,

$$
\mathcal{E}^{\prime}(\Omega)=\left\{u \in \mathcal{D}^{\prime}(\Omega) ; \operatorname{supp}(u) \text { é compacto }\right\}
$$

Teorema 1.38. Seja $u \in \mathcal{E}^{\prime}(\Omega)$. Existe um único funcional linear $\tilde{u}: C^{\infty}(\Omega) \longrightarrow \mathbb{C}$ tal que satisfaz a seguintes condições:
i) $\tilde{u}(\varphi)=u(\varphi)$, para todo $\varphi \in C_{c}^{\infty}(\Omega)$.
ii) $\tilde{u}(\varphi)=0$ se $\varphi \in C^{\infty}(\Omega)$ e $\operatorname{supp}(\varphi) \cap \operatorname{supp}(u)=\emptyset$.

Demonstração. Mostremos a unicidade. Para isto, suponha que existem dois funcionais lineares $\tilde{u}_{1}$ e $\tilde{u}_{2}$ que satisfazem $i$ ) e $i i$ ).

Como $\operatorname{supp}(u) \subset \Omega$ é compacto, então podemos considerar $\psi \in C_{c}^{\infty}(\Omega)$ tal que $\psi \equiv 1 \mathrm{em}$ 
uma vizinhança de $\operatorname{supp}(u)$.

Agora, seja $\varphi \in C^{\infty}(\Omega)$, temos que

$$
\varphi=\varphi+\varphi \psi-\varphi \psi=\varphi \psi+(1-\psi) \varphi=\varphi_{1}+\varphi_{2}
$$

onde $\varphi_{1}=\varphi \psi \in C_{c}^{\infty}(\Omega), \varphi_{2}=(1-\psi) \varphi \in C^{\infty}(\Omega)$ e $\operatorname{supp}\left(\varphi_{2}\right) \cap \operatorname{supp}(u)=\emptyset$. Logo

$$
\begin{aligned}
\tilde{u}_{1}(\varphi) & =\tilde{u}\left(\varphi_{1}\right)+\tilde{u}\left(\varphi_{2}\right)=\tilde{u}\left(\varphi_{1}\right)=u\left(\varphi_{1}\right) \\
& =\tilde{u}_{2}\left(\varphi_{1}\right)=\tilde{u}_{2}\left(\varphi_{1}\right)+\tilde{u}_{2}\left(\varphi_{2}\right)=\tilde{u}_{2}(\varphi)
\end{aligned}
$$

onde na segunda e quinta iguladade usamos a condição $i i$ ) e na terceira e quarta igualdade usamos a condição $i$ ). Portanto temos que $\tilde{u}_{1}=\tilde{u}_{2}$

Para mostrar a existência, defina $\tilde{u}: C^{\infty}(\Omega) \longrightarrow \mathbb{C}$ da seguinte forma $\tilde{u}(\varphi)=u\left(\varphi_{1}\right)$, onde $\varphi=\varphi_{1}+\varphi_{2}$ é qualquer descomposição de $\varphi \operatorname{com} \varphi_{1} \in C_{c}^{\infty}(\Omega), \varphi_{2} \in C^{\infty}(\Omega)$ e $\operatorname{supp}\left(\varphi_{2}\right) \cap \operatorname{supp}(u)=\emptyset$.

Vejamos se $\tilde{u}$ está bem definida. De fato, se $\varphi=\varphi_{1}^{\prime}+\varphi_{2}^{\prime}=\varphi_{1}+\varphi_{2}$, então $\varphi_{1}-\varphi_{1}^{\prime}=\varphi_{2}^{\prime}-\varphi_{2}$, daí segue que

$$
\operatorname{supp}\left(\varphi_{1}-\varphi_{1}^{\prime}\right)=\operatorname{supp}\left(\varphi_{2}^{\prime}-\varphi_{2}\right) \quad \text { e } \quad \operatorname{supp}\left(\varphi_{2}^{\prime}-\varphi_{2}\right) \cap \operatorname{supp}(u)=\emptyset
$$

$\operatorname{pois} \operatorname{supp}\left(\varphi_{2}^{\prime}\right) \cap \operatorname{supp}(u)=\emptyset$ e $\operatorname{supp}\left(\varphi_{2}\right) \cap \operatorname{supp}(u)=\emptyset$. Assim obtemos que

$$
\operatorname{supp}\left(\varphi_{1}-\varphi_{1}^{\prime}\right) \cap \operatorname{supp}(u)=\emptyset
$$

o qual implica que

$$
u\left(\varphi_{1}-\varphi_{1}^{\prime}\right)=0
$$

Portanto $u\left(\varphi_{1}\right)=u\left(\varphi_{1}^{\prime}\right)$. Ou seja $\tilde{u}$ está bem definida.

Para a linearidade de $\tilde{u} \operatorname{sejam} \varphi, \phi \in C^{\infty}(\Omega)$ e $\alpha \in \mathbb{C}$, então

$$
\tilde{u}(\varphi+\alpha \phi)=u\left(\varphi_{1}+\alpha \phi_{1}\right)=u\left(\varphi_{1}\right)+\alpha u\left(\phi_{1}\right)=\tilde{u}(\varphi)+\alpha \tilde{u}(\phi) .
$$

Portanto $\tilde{u}$ é linear.

Finalmente para verificar $i$ ) basta tomar a descomposição $\varphi=\varphi+0$ onde $\varphi \in C_{c}^{\infty}(\Omega)$. E para verificar $i i)$ basta tomar a descomposição $\varphi=0+\varphi$, onde $\varphi \in C^{\infty}(\Omega)$ com $\operatorname{supp}(\varphi) \cap \operatorname{supp}(u)=\emptyset$. 


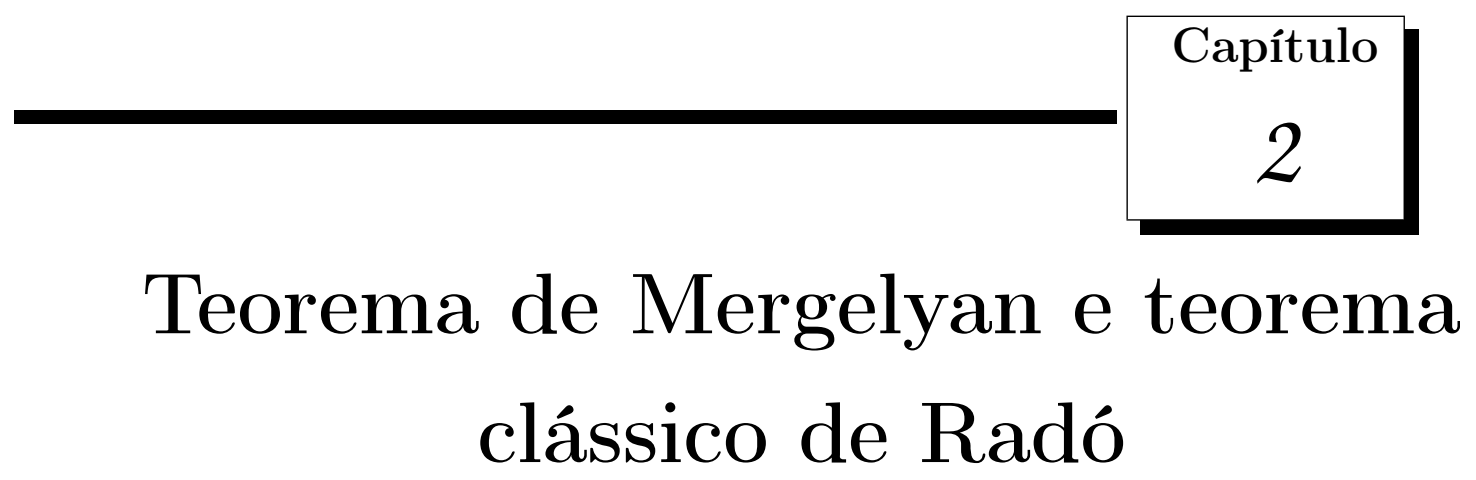

Neste capítulo apresentaremos algumas propriedades básicas sobre funções holomorfas e funções harmônicas e principalmente mostraremos dois teoremas de grande importância para este trabalho, o Teorema de Mergelyam e o Teorema clássico de Radó. Alguns resultados serão somente enunciados, sendo omitidas suas demonstrações, podendo estas ser encontradas em Conway [2] ou Rudin [12].

Vamos começar este capítulo lembrando a definição de diferenciabilidade de uma função de várias variáveis reais.

Definição 2.1. Suponha que $V$ é um subconjunto aberto de $\mathbb{R}^{n}, F: V \longrightarrow \mathbb{R}^{n}$ uma função, $x \in V$ e $T$ um operador linear sobre $\mathbb{R}^{n}$.

Se para todo $\epsilon>0$ existe $\delta>0$ tal que a desigualdade

$$
\|F(x+h)-F(x)-T h\| \leq \epsilon\|h\|
$$

se satisfaz para todo $h \in \mathbb{R}^{n}$ com $\|h\|<\delta$ e $x+h \in V$, dizemos que $F$ é diferenciável em $x$ e definimos

$$
F^{\prime}(x)=T
$$

O operador linear $F^{\prime}(x)$ é chamado de derivada de $F$ em $x$. O termo diferencial é freqüentemente usado para $F^{\prime}(x)$. Portanto pode-se falar também que $F$ tem um diferencial em $x$, ou que o diferencial de $F$ existe em $x$.

Dizemos que $\mathrm{F}$ é diferenciável em $V$ se $F$ é diferenciável em todo ponto de $V$. 
Antes de falar sobre funções holomorfas, vamos fixar primeiro algumas notações. Se $r>0$ e $a$ é numero complexo, então

$$
D(a, r)=\{z ;|z-a|<r\}
$$

é o disco aberto com centro em $a$ e raio $r . \bar{D}(a, r)$ é o fecho de $D(a, r)$ e $D(0,1)$ denotará o disco unitário.

Além disso, $\Omega$ denotará sempre um subconjunto aberto do plano complexo $\mathbb{C}$.

Definição 2.2. Seja $f: \Omega \longrightarrow \mathbb{C}$ uma função complexa definida em $\Omega$. Se $z_{0} \in \Omega$ e se

$$
\lim _{z \rightarrow z_{0}} \frac{f(z)-f\left(z_{0}\right)}{z-z_{0}}
$$

existe, denotamos este limite por $f^{\prime}\left(z_{0}\right)$ e chamamos de derivada de $f$ em $z_{0}$.

Se $f^{\prime}\left(z_{0}\right)$ existe para todo $z_{0} \in \Omega$, dizemos que $f$ é holomorfa (ou analítica) em $\Omega$. A classe de todas as funçôes holomorfas em $\Omega$ sera denotada por $H(\Omega)$.

Se na definição anterior nós olhamos a função complexa $f$ como uma transformação que leva $\Omega$ em $\mathbb{R}^{2}$ e assumimos que $f$ tem um diferencial em algum ponto $z_{0} \in \Omega$, no sentido da Definição 2.1. Para mais simplicidade, suponha que $z_{0}=f\left(z_{0}\right)=0$. Logo nossa hipótese de diferenciabilidade é equivalente à existência de dois números complexos $\alpha$ e $\beta$ (as derivadas parciais de $f$ em relação a $x$ e $y$ em $z_{0}=0$ ) tais que

$$
f(z)=\alpha x+\beta y+\eta(z) z \operatorname{com} z=x+i y
$$

onde $\eta(z) \rightarrow 0$ quando $z \rightarrow 0$.

Como $2 x=z+\bar{z}$ e $2 i y=z-\bar{z}$, a equação (2.3) pode ser reescrita na forma seguinte

$$
f(z)=\frac{\alpha-i \beta}{2} z+\frac{\alpha+i \beta}{2} \bar{z}+\eta(z) z
$$

Isso motiva a introdução dos seguintes operadores diferenciais

$$
\partial=\frac{1}{2}\left(\frac{\partial}{\partial x}-i \frac{\partial}{\partial y}\right) \text { e } \bar{\partial}=\frac{1}{2}\left(\frac{\partial}{\partial x}+i \frac{\partial}{\partial y}\right)
$$

Agora a equação (2.4) torna-se

$$
\frac{f(z)}{z}=(\partial f)(0)+(\bar{\partial} f)(0) \cdot \frac{\bar{z}}{z}+\eta(z) \operatorname{com} z \neq 0 .
$$

Para $z$ real, $\bar{z} / z=1$; para $z$ imaginário puro, $\bar{z} / z=-1$. Assim $f(z) / z$ tem limite em 0 
se e somente se $(\bar{\partial} f)(0)=0$, e obtemos a seguinte caracterização das funções holomorfas.

Teorema 2.3. Suponha que $f$ é uma função complexa definida em $\Omega$ a qual tem um diferencial em todo ponto de $\Omega$. Então $f \in H(\Omega)$ se e somente se a equação de CauchyRiemann

$$
(\bar{\partial} f)(z)=0
$$

se satisfaz para todo $z \in \Omega$. Nesse caso, temos

$$
f^{\prime}(z)=(\partial f)(z)
$$

Se escrevemos $f=u+i v$, onde $u$ e $v$ são funções a valores reais, a equação de Cauchy-Riemann (2.7) é equivalente ao par de equações seguintes

$$
\left\{\begin{array}{l}
\frac{\partial u}{\partial x}=\frac{\partial v}{\partial y} \\
\frac{\partial u}{\partial y}=-\frac{\partial v}{\partial x}
\end{array}\right.
$$

Estas são as conhecidas equações de Cauchy-Riemann.

Definição 2.4. Seja $f: \Omega \longrightarrow \mathbb{C}$ uma função complexa tal que $f_{x x}$ e $f_{y y}$ existem em todo ponto de $\Omega$. O Laplaciano de $f$ é definido por

$$
\Delta f=f_{x x}+f_{y y}
$$

Se $f$ é contínua em $\Omega$ e se $\Delta f=0$ em todo ponto de $\Omega$, então dizemos que $f$ é harmônica em $\Omega$.

Vamos enumerar algumas consequências imediatas desta definição

1) Desde que o Laplaciano de uma função real é real (se este existe), é claro que uma função complexa é harmônica em $\Omega$ se e somente se sua parte real e imaginaria são harmônicas em $\Omega$.

2) Observe que se $f$ tem derivadas de segunda ordem contínuas, então temos que

$$
\Delta f=4 \partial \bar{\partial} f=4 \bar{\partial} \partial f
$$

3) Lembremos que $f$ é holomorfa em $\Omega$ se e somente se $\bar{\partial} f=0$ em $\Omega$. Assim para $f$ com derivadas de segunda ordem contínuas temos que, $f$ é harmônica se e somente se $\partial f$ é holomorfa. Em particular toda função holomorfa é harmônica. 
Um dos aspectos importantes sobre funções harmônicas é que elas surgem como funções que resolvem um problema de valor em fronteira para funções holomorfas, conhecido como o problema de Dirichlet. Um exemplo é o problema de encontrar uma função contínua em um disco fechado, que assume determinados valores conhecidos na fronteira do disco e é harmônica no interior do disco. O seguinte teorema afirma que o problema de Dirichlet pode-se resolver para o disco unitário.

Teorema 2.5. Seja $D=D(0,1)$ o disco unitário e suponha que $f: \partial D \longrightarrow \mathbb{R}$ é uma função contínua. Então existe uma única função continua $u: \bar{D} \longrightarrow \mathbb{R}$ tal que

a) $u(z)=f(z)$, para todo $z \in \partial D$.

b) u é harmônica em $D$.

Demonstração. A demonstração deste teorema pode-se encontar em [2].

Vamos definir agora a integral de uma função complexa ao longo de uma curva. Para isto precisamos das seguintes definições.

Definição 2.6. Uma curva no plano complexo $\mathbb{C}$ é uma função contínua

$$
\gamma:[a, b] \subset \mathbb{R} \longrightarrow \mathbb{C}
$$

Se $t \in[a, b]$, indicamos o ponto $\gamma(t) \in \mathbb{C}$ por

$$
\gamma(t)=z(t)=x(t)+i y(t)
$$

Denotamos por $\gamma^{*}$ o conjunto $\{\gamma(t) ; t \in[a, b]\}$ que é chamado traço de $\gamma$.

\section{Observação 2.7 .}

1) As funções reais $x(t)$ e $y(t)$, componentes da curva $\gamma$, são contínuas.

2) Os pontos $\gamma(a)$ e $\gamma(b)$ da curva $\gamma$ são chamados, respectivamente, ponto inicial e final da curva ou simplesmente extremos da curva. A curva $\gamma$ é fechada se $\gamma(a)=\gamma(b)$. Uma curva fechada $\gamma$ é simples se $a \leq t<t^{\prime} \leq b$ e $\gamma(t)=\gamma\left(t^{\prime}\right)$ implica $t=a$ e $t^{\prime}=b$.

Definição 2.8. Uma curva $\gamma:[a, b] \longrightarrow \mathbb{C} e ́$ chamada de suave se $\gamma^{\prime}(t)$ existe e é contínua para todo $t \in[a, b]$. Além disso, $\gamma$ é suave por partes se existe uma partição de $[a, b], a=t_{0}<t_{1}<\ldots<t_{n}=b$ tal que $\gamma$ é suave em cada subintervalo $\left[t_{j-1}, t_{j}\right]$, $1 \leq j \leq n$. 
Dizer que a função $\gamma:[a, b] \longrightarrow \mathbb{C}$ tem derivada $\gamma^{\prime}(t)$ para todo ponto $t \in[a, b]$ significa que

$$
\lim _{h \rightarrow 0} \frac{\gamma(t+h)-\gamma(t)}{h}=\gamma^{\prime}(t)
$$

existe para $a<t<b$ e que os limites laterais à direita e à esquerda existem para $t=a \mathrm{e}$ $t=b$, respectivamente.

Definição 2.9. Seja $\gamma:[a, b] \longrightarrow \mathbb{C}$ uma curva suave por partes $e f: \gamma^{*} \longrightarrow \mathbb{C}$ uma função contínua, então a integral de $f$ ao longo de $\gamma$ é dada por

$$
\int_{\gamma} f(z) d z=\int_{a}^{b} f(\gamma(t)) \gamma^{\prime}(t) d t
$$

Pode-se mostrar que a integral (2.10) é independente da troca de parâmetro.

Um caso especial é dado pela curva $\gamma:[0,2 \pi] \longrightarrow \mathbb{C}$ definida por

$$
\gamma(t)=a+r e^{i t}
$$

onde $a$ é um número complexo e $r>0$. Esta curva é chamada o círculo positivamente orientado com centro em $a$ e raio $r$, logo a integral (2.10) fica da seguinte forma

$$
\int_{\gamma} f(z) d z=i r \int_{0}^{2 \pi} f\left(a+r e^{i t}\right) e^{i t} d t
$$

além disso, o comprimento de $\gamma$ é $2 \pi r$.

Vamos enunciar sem demostrar a regra de Leibniz.

Teorema 2.10. Seja $\varphi:[a, b] \times[c, d] \longrightarrow \mathbb{C}$ uma função contínua e defina $g:[c, d] \longrightarrow \mathbb{C}$ por

$$
g(t)=\int_{a}^{b} \varphi(s, t) d s .
$$

Então g é contínua. Além disso, se $\frac{\partial \varphi}{\partial t}:[a, b] \times[c, d] \longrightarrow \mathbb{C}$ existe e for contínua, então $g$ será continuamente diferenciável $e$

$$
g^{\prime}(t)=\int_{a}^{b} \frac{\partial \varphi}{\partial t}(s, t) d s
$$

Demonstração. Para a demonstração deste teorema ver [2]. 
Este resultado pode ser usado para mostrar a igualdade seguinte

$$
\int_{0}^{2 \pi} \frac{e^{i s}}{e^{i s}-z} d s=2 \pi \text { se }|z|<1 .
$$

De fato, seja $\varphi(s, t)=\frac{e^{i s}}{e^{i s}-t z}$ para $0 \leq s \leq 2 \pi, 0 \leq t \leq 1$. Como $|z|<1$, temos que $\varphi$ é continuamente diferenciável. Logo usando o Teorema 2.10 vem que

$$
g(t)=\int_{0}^{2 \pi} \varphi(s, t) d s=\int_{0}^{2 \pi} \frac{e^{i s}}{e^{i s}-t z} d s
$$

é continuamente diferenciável. Além disso, temos

$$
g(0)=\int_{0}^{2 \pi} \varphi(s, 0) d s=2 \pi .
$$

Por outro lado $\frac{\partial \varphi}{\partial t}(s, t)=\frac{z e^{i s}}{\left(e^{i s}-t z\right)^{2}}$ de onde obtemos o seguinte

$$
g^{\prime}(t)=\int_{0}^{2 \pi} \frac{\partial \varphi}{\partial t}(s, t) d s=\int_{0}^{2 \pi} \frac{z e^{i s}}{\left(e^{i s}-t z\right)^{2}} d s
$$

mas para $t$ fixado, temos que a função $\phi(s)=i z\left(e^{i s}-t z\right)^{-1}$ é diferenciável e $\phi^{\prime}(s)=$ $\frac{\partial \varphi}{\partial t}(s, t)$. Portanto

$$
g^{\prime}(t)=\phi(2 \pi)-\phi(0)=0
$$

o qual implica que $g$ é constante. Como $g$ é constante e $g(0)=2 \pi$, temos que $g(1)=2 \pi$ e fica mostrada a igualdade (2.11).

Usando o Teorema 2.10 e a igualdade (2.11) vamos mostrar a seguinte versão da fórmula integral de Cauchy.

Proposição 2.11. Seja $f: \Omega \longrightarrow \mathbb{C}$ holomorfa e suponha $\bar{D}(a, r) \subset \Omega$ com $r>0$. Se $\gamma(s)=a+r e^{i s}, 0 \leq s \leq 2 \pi$, então

$$
f(z)=\frac{1}{2 \pi i} \int_{\gamma} \frac{f(w)}{w-z} d w, \quad \text { para todo } z \in D(a, r) .
$$

Demonstração. Cosiderando $\Omega_{1}=\left\{\frac{1}{r}(z-a) ; z \in \Omega\right\}$ e a função $g: \Omega_{1} \longrightarrow \mathbb{C}$ definida por $g(z)=f(a+r z)$, podemos assumir sem perda de generalidade que $a=0$ e $r=1$. Ou seja, podemos assumir que $\bar{D}(0,1) \subset \Omega$. 
Para $z$ fixo com $|z|<1$ devemos mostrar que

$$
f(z)=\frac{1}{2 \pi i} \int_{\gamma} \frac{f(w)}{w-z} d w=\frac{1}{2 \pi} \int_{0}^{2 \pi} \frac{f\left(e^{i s}\right)}{e^{i s}-z} e^{i s} d s
$$

ou equivalentemente

$$
\int_{0}^{2 \pi}\left[\frac{e^{i s} f\left(e^{i s}\right)}{e^{i s}-z}-f(z)\right] d s=0
$$

De fato, para $0 \leq t \leq 1$ e $0 \leq s \leq 2 \pi$ considere a seguinte função

$$
\varphi(s, t)=\frac{e^{i s} f\left((1-t) z+t e^{i s}\right)}{e^{i s}-z}-f(z) .
$$

Como $\left|(1-t) z+t e^{i s}\right|=\left|z+t\left(e^{i s}-z\right)\right| \leq 1$, temos que $\varphi$ está bem definida e é continuamente diferenciável. Logo aplicando o Teorema 2.10 obtemos que $g(t)=\int_{0}^{2 \pi} \varphi(s, t) d s$ é continuamente diferenciável e

$$
g^{\prime}(t)=\int_{0}^{2 \pi} \frac{\partial \varphi}{\partial t}(s, t) d s=\int_{0}^{2 \pi} e^{i s} f^{\prime}\left((1-t) z+t e^{i s}\right) d s .
$$

Além disso,

$$
\begin{aligned}
g(0) & =\int_{0}^{2 \pi} \varphi(s, 0) d s=\int_{0}^{2 \pi}\left[\frac{e^{i s} f(z)}{e^{i s}-z}-f(z)\right] d s \\
& =f(z) \int_{0}^{2 \pi} \frac{e^{i s}}{e^{i s}-z} d s-2 \pi f(z) \underbrace{=}_{\text {por } 2.11} 0
\end{aligned}
$$

Por outro lado, para $0<t \leq 1$, temos que a função $\phi(s)=-i t^{-1} f\left((1-t) z+t e^{i s}\right)$ é diferenciável e $\phi^{\prime}(s)=\frac{\partial \varphi}{\partial t}(s, t)$, logo temos

$$
g^{\prime}(t)=\phi(2 \pi)-\phi(0)=0 \quad \text { para } 0<t \leq 1
$$

e como $g^{\prime}$ é contínua, segue que $g^{\prime}(0)=0$. Portanto $g$ é constante. Logo vem que

$$
0=g(1)=\int_{0}^{2 \pi}\left[\frac{e^{i s} f\left(e^{i s}\right)}{e^{i s}-z}-f(z)\right] d s
$$

o qual implica que

$$
f(z)=\frac{1}{2 \pi} \int_{0}^{2 \pi} \frac{e^{i s} f\left(e^{i s}\right)}{e^{i s}-z} d s
$$


Observação 2.12. Se fazemos a substituição $w=a+r e^{i s}, s \in[0,2 \pi]$ na fórmula integral de Cauchy (2.12), obtemos que as funções holomorfas satisfazem a seguinte igualdade

$$
f(a)=\frac{1}{2 \pi} \int_{0}^{2 \pi} f\left(a+r e^{i s}\right) d s
$$

Esta igualdade é chamada de propriedade do valor médio.

As partes reais e imaginárias da $f$ satisfazem também a mesma propriedade.

Um outro resultado importante na teoria das funções holomorfas é o chamado Teorema do módulo máximo, que enunciamos a continuação.

Teorema 2.13. (do módulo máximo) Sejam $\Omega \subset \mathbb{C}$ aberto e conexo e $f: \Omega \longrightarrow \mathbb{C}$ holomorfa. Suponha que existe $a \in \Omega$ tal que $|f(a)| \geq|f(z)|$ para todo $z \in \Omega$, então $f$ é constante.

Demonstração. Para a demonstração deste teorema pode-se ver [2] ou [12].

\section{Observação 2.14 .}

1) O Teorema do módulo máximo afirma que o módulo de uma função holomorfa não constante, não tem máximos relativos.

2) Suponha que a função $f$ é holomorfa em $\Omega \subset \mathbb{C}$, onde $\Omega$ é aberto, conexo e limitado, e contínua em $\bar{\Omega}$. Então a função $|f|$ assume seu máximo valor em algum ponto de $\bar{\Omega}$. O Teorema do módulo máximo pode ser usado então para afirmar que este máximo é sempre assumido sobre a fronteira de $\Omega(\partial \Omega)$.

\subsection{O Teorema de Mergelyan}

Seja $K$ um conjunto compacto no plano complexo e $\stackrel{o}{K}$ o interior de $K$, denotemos por $P(K)$ o conjunto de todas as funções sobre $K$ as quais são limites uniformes de polinômios em $z$. Uma questão seria: quais funções pertencem a $P(K)$ ?.

Duas condições necessárias vêm à mente imediatamente: se $f \in P(K)$, então $f \in \mathcal{C}(K)$ e $f \in H(\stackrel{o}{K})$. A questão é se estas condições necessárias também são suficientes. A resposta é negativa sempre que $K$ separe o plano, ou seja, quando o complementar de $K$ é não conexo. Por outro lado se $K$ é um intervalo no eixo real (em tal caso $\stackrel{o}{K}=\emptyset$ ), o Teorema de aproximação de Weierstrass afirma que $P(K)=\mathcal{C}(K)$, logo a resposta é positiva se $K$ é um intervalo. 
O Teorema de Runge também aponta nessa direção, pois afirma que para conjuntos compactos $K$ que não separam o plano: $P(K)$ contém pelo menos todas aquelas funções $f \in \mathcal{C}(K)$ que têm extensão holomorfa para algum conjunto aberto $\Omega$ contendo $K$.

Agora veremos um outro teorema chamado de Teorema de Mergelyan o qual afirma sem qualquer hipótese supérflua que a condição necessária mencionada acima também é suficiente se $K$ não separa o plano.

Antes de enunciar e mostrar o Teorema de Mergelyan, vamos dar alguns resultados que serão usados na demonstração.

Lema 2.15. Suponha que $D$ é um disco aberto de raio $r>0, E \subset D, E$ é compacto $e$ conexo, $\Omega=S^{2} \backslash E$ é conexo e o diâmetro de $E$ é ao menos $r$. Então existe uma função $g \in H(\Omega)$ e uma constante $b$ com a seguinte propiedade: se

$$
Q(\zeta, z)=g(z)+(\zeta-b) g^{2}(z)
$$

as desigualdades

$$
\begin{gathered}
|Q(\zeta, z)|<\frac{100}{r} \\
\left|Q(\zeta, z)-\frac{1}{z-\zeta}\right|<\frac{1,000 r^{2}}{|z-\zeta|^{3}}
\end{gathered}
$$

são satisfeitas, para todo $z \in \Omega$ e para todo $\zeta \in D$.

Demonstração. Para a demonstração consulte [12].

Se $\bar{\partial}=\frac{1}{2}\left(\frac{\partial}{\partial x}+i \frac{\partial}{\partial y}\right)$ é o operador de Cauchy-Riemann, temos o seguinte lema

Lema 2.16. Suponha que $f \in C_{c}^{1}\left(\mathbb{R}^{2}\right)$. Então a seguinte fórmula se satisfaz

$$
f(z)=-\frac{1}{\pi} \iint_{\mathbb{R}^{2}} \frac{(\bar{\partial} f)(\zeta)}{\zeta-z} d \xi d \eta \quad, \quad(\zeta=\xi+i \eta)
$$

Demonstração. Considere $\varphi(r, \theta)=f\left(z+r e^{i \theta}\right)$, $\operatorname{com} r>0$ e $\theta$ real. O operador $\bar{\partial}$ em coordenadas polares é dado por

$$
\bar{\partial}=\frac{1}{2} e^{i \theta}\left(\frac{\partial}{\partial r}+\frac{i}{r} \frac{\partial}{\partial \theta}\right) .
$$

Se escrevemos $\zeta=z+r e^{i \theta}$, temos que

$$
(\bar{\partial} f)(\zeta)=\frac{1}{2} e^{i \theta}\left(\frac{\partial}{\partial r} \varphi(r, \theta)+\frac{i}{r} \frac{\partial}{\partial \theta} \varphi(r, \theta)\right)
$$


Por outro lado, observe que, para cada $r>0$ a função $\varphi$ é periódica em $\theta$ de período $2 \pi$, ou seja, $\varphi(r, \theta)=\varphi(r, \theta+2 \pi)$. Portanto, a integral de $\partial \varphi / \partial \theta$ é zero em $[0,2 \pi]$.

Logo temos

$$
\begin{aligned}
-\frac{1}{\pi} \iint_{\mathbb{R}^{2}} \frac{(\bar{\partial} f)(\zeta)}{\zeta-z} d \xi d \eta & =\lim _{\epsilon \rightarrow 0}-\frac{1}{2 \pi} \int_{\epsilon}^{\infty} \int_{0}^{2 \pi} \frac{e^{i \theta}\left(\frac{\partial \varphi}{\partial r}+\frac{i}{r} \frac{\partial \varphi}{\partial \theta}\right)}{z+r e^{i \theta}-z} r d \theta d r \\
& =\lim _{\epsilon \rightarrow 0}-\frac{1}{2 \pi} \int_{\epsilon}^{\infty} \int_{0}^{2 \pi}\left(\frac{\partial \varphi}{\partial r}+\frac{i}{r} \frac{\partial \varphi}{\partial \theta}\right) d \theta d r \\
& =\lim _{\epsilon \rightarrow 0}-\frac{1}{2 \pi} \int_{0}^{2 \pi} \int_{\epsilon}^{\infty} \frac{\partial \varphi}{\partial r} d r d \theta \\
& =\lim _{\epsilon \rightarrow 0}-\frac{1}{2 \pi} \int_{0}^{2 \pi}[\varphi(r, \theta)]_{\epsilon}^{\infty} d \theta \\
& =\frac{1}{2 \pi} \lim _{\epsilon \rightarrow 0} \int_{0}^{2 \pi} \varphi(\epsilon, \theta) d \theta \\
& =\frac{1}{2 \pi} \int_{0}^{2 \pi} \lim _{\epsilon \rightarrow 0} \varphi(\epsilon, \theta) d \theta \\
& =f(z),
\end{aligned}
$$

A última igualdade é satisfeita porque $\varphi(\epsilon, \theta)$ converge uniformemente a $f(z)$ quando $\epsilon \rightarrow 0$ e $\frac{1}{2 \pi} \int_{0}^{2 \pi} d \theta=1$. Portanto, obtemos a igualdade desejada

$$
f(z)=-\frac{1}{\pi} \iint_{\mathbb{R}^{2}} \frac{(\bar{\partial} f)(\zeta)}{\zeta-z} d \xi d \eta
$$

Teorema 2.17. (da extensão de Tietze)

Suponha que $K$ é um subconjunto compacto de um espaço Hausdorff localmente compacto $X$ e $f \in C(K)$, então existe $F \in C_{c}^{0}(X)$ tal que $F(x)=f(x)$ para todo $x \in K$.

Demonstração. Para a demonstração consulte [12].

Teorema 2.18. (de Mergelyan)

Se $K$ é um conjunto compacto no plano com o seu complementar conexo, se $f$ é uma função complexa contínua sobre $K$ a qual é holomorfa no interior de $K$, e se $\epsilon>0$, então existe um polinômio $P$ tal que $|f(z)-P(z)|<\epsilon$, para todo $z \in K$.

Demonstração. Como $f \in C(K)$, com $K \subset \mathbb{C}$ compacto, $\mathbb{C}$ é Hausdorff e localmente compacto então pelo Teorema da extensão de Tietze, existe uma função $F \in C_{c}(\mathbb{C})$ tal que $F(x)=f(x)$, para todo $x \in K$. Vamos fixar uma tal extensão e denotaremos ela ainda por $f$. 
Para qualquer $\delta>0$, definimos

$$
\omega(\delta)=\sup \left\{\left|f\left(z_{2}\right)-f\left(z_{1}\right)\right| ;\left|z_{2}-z_{1}\right| \leq \delta\right\}
$$

Como $f$ é uniformemente contínua, temos que

$$
\lim _{\delta \rightarrow 0} \omega(\delta)=0
$$

Daqui em diante, $\delta$ será fixado. Vamos provar que existe um polinômio $P$ tal que

$$
|f(z)-P(z)|<10000 \omega(\delta) \quad, \quad \text { para } z \in K \text {. }
$$

e usando (2.18) o teorema ficara provado.

Primeiro construiremos uma função $\Phi \in C_{c}^{1}\left(\mathbb{R}^{2}\right)$ tal que para todo $z$ satisfaz as seguintes propriedades:

$$
\begin{gathered}
|f(z)-\Phi(z)| \leq \omega(\delta) \\
|(\bar{\partial} \Phi)(z)|<\frac{2 \omega(\delta)}{\delta}
\end{gathered}
$$

e

$$
\Phi(z)=-\frac{1}{\pi} \iint_{X} \frac{(\bar{\partial} \Phi)(\zeta)}{\zeta-z} d \xi d \eta \quad \operatorname{com} \quad \zeta=\xi+i \eta
$$

onde $X=\left\{z \in \operatorname{supp}(\Phi) ; d\left(z, K^{c}\right) \leq \delta\right\}$.

De fato, considere a seguinte função

$$
a(r)=\left\{\begin{array}{cll}
\frac{3}{\pi \delta^{2}}\left(1-\frac{r^{2}}{\delta^{2}}\right)^{2} & \text { se } & 0 \leq r \leq \delta \\
0 & \text { se } & r>\delta
\end{array}\right.
$$

cuja derivada é dada por

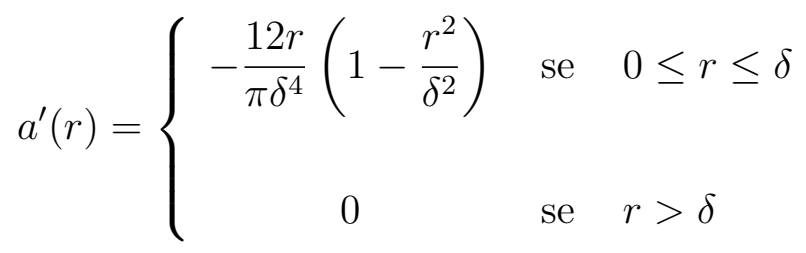

Então $a \in C_{c}^{1}(\mathbb{R})$, pois $a, a^{\prime}$ são contínuas e, além disso, $\operatorname{supp}(a)=[0, \delta]$ que é compacto. 
Logo, para todo $z \in \mathbb{R}^{2}$ definamos

$$
A(z)=a(|z|)=\left\{\begin{array}{cll}
\frac{3}{\pi \delta^{2}}\left(1-\frac{|z|^{2}}{\delta^{2}}\right)^{2} & \text { se } & 0 \leq|z| \leq \delta \\
0 & \text { se } & |z|>\delta
\end{array}\right.
$$

e temos que $A \in C_{c}^{1}\left(\mathbb{R}^{2}\right)$.

Vamos fazer as seguintes afirmações

$$
\begin{gathered}
\iint_{\mathbb{R}^{2}} A=1 \\
\iint_{\mathbb{R}^{2}} \bar{\partial} A=0 \\
\iint_{\mathbb{R}^{2}}|\bar{\partial} A|=\frac{24}{15 \delta}<\frac{2}{\delta}
\end{gathered}
$$

Para mostrar estas afirmações trabalharemos em coordenadas polares.

$$
\begin{gathered}
\iint_{\mathbb{R}^{2}} A=\int_{0}^{2 \pi} \int_{0}^{\delta} \frac{3}{\pi \delta^{2}}\left(1-\frac{r^{2}}{\delta^{2}}\right)^{2} r d r d \theta=\frac{3}{\pi \delta^{2}} \int_{0}^{2 \pi} \int_{0}^{\delta}\left(r-\frac{2 r^{3}}{\delta^{2}}+\frac{r^{5}}{\delta^{4}}\right) d r d \theta \\
=\left.\frac{3}{\pi \delta^{2}} \int_{0}^{2 \pi}\left(\frac{r^{2}}{2}-\frac{r^{4}}{2 \delta^{2}}+\frac{r^{6}}{6 \delta^{4}}\right)\right|_{0} ^{\delta} d \theta=\frac{1}{2 \pi} \int_{0}^{2 \pi} d \theta=1 . \\
\iint_{\mathbb{R}^{2}} \bar{\partial} A=\int_{0}^{\delta} \int_{0}^{2 \pi} \frac{1}{2} e^{i \theta}\left[\frac{\partial A}{\partial r}+\frac{i}{r} \frac{\partial A}{\partial \theta}\right] r d \theta d r=\int_{0}^{\delta} \int_{0}^{2 \pi} \frac{1}{2} e^{i \theta}\left[-\frac{12}{\pi \delta^{4}}\left(r-\frac{r^{3}}{\delta^{2}}\right)\right] r d \theta d r \\
=-\frac{6}{\pi \delta^{4}} \int_{0}^{\delta}\left(r^{2}-\frac{r^{4}}{\delta^{2}}\right) d r \int_{0}^{2 \pi} e^{i \theta} d \theta=-\frac{6}{i \pi \delta^{4}} \int_{0}^{\delta}\left(r^{2}-\frac{r^{4}}{\delta^{2}}\right)\left[e^{2 \pi i}-1\right] d r=0 \\
\iint_{\mathbb{R}^{2}} \bar{\partial} A \mid=\int_{0}^{2 \pi} \int_{0}^{\delta} \frac{6}{\pi \delta^{4}}\left(r-\frac{r^{3}}{\delta^{2}}\right) r d r d \theta=\frac{6}{\pi \delta^{4}} \int_{0}^{2 \pi} \int_{0}^{\delta}\left(r^{2}-\frac{r^{4}}{\delta^{2}}\right) d r d \theta \\
=\left.\frac{6}{\pi \delta^{4}} \int_{0}^{2 \pi}\left(\frac{r^{3}}{3}-\frac{r^{5}}{5 \delta^{2}}\right)\right|_{0} ^{\delta} d \theta=\frac{12}{15 \pi \delta} \int_{0}^{2 \pi} d \theta=\frac{24}{15 \delta}<\frac{2}{\delta} .
\end{gathered}
$$


Defina agora a seguinte função

$$
\Phi(z)=\iint_{\mathbb{R}^{2}} f(z-\zeta) A(\zeta) d \xi d \eta=\iint_{\mathbb{R}^{2}} A(z-\zeta) f(\zeta) d \xi d \eta=(f * A)(z) .
$$

Onde $\zeta=\xi+i \eta$. Como $f$ e $A$ têm suporte compacto, então $\Phi$ também tem suporte compacto, pois, $\operatorname{supp}(\Phi) \subset \operatorname{supp}(f)+\operatorname{supp}(A)$. Além disso, temos

$$
\begin{aligned}
\Phi(z)-f(z) & =\iint_{\mathbb{R}^{2}} f(z-\zeta) A(\zeta) d \xi d \eta-f(z) \\
& =\iint_{\mathbb{R}^{2}} f(z-\zeta) A(\zeta) d \xi d \eta-\iint_{\mathbb{R}^{2}} f(z) A(\zeta) d \xi d \eta \\
& =\iint_{\mathbb{R}^{2}}[f(z-\zeta)-f(z)] A(\zeta) d \xi d \eta
\end{aligned}
$$

e como $A(\zeta)=0$ quando $|\zeta|>\delta$, então segue que

$$
\begin{aligned}
|\Phi(z)-f(z)| & \leq \iint_{\mathbb{R}^{2}}|f(z-\zeta)-f(z) \| A(\zeta)| d \xi d \eta \\
& \leq \sup _{|\zeta| \leq \delta}\{|f(z-\zeta)-f(z)|\} \iint_{\mathbb{R}^{2}} A(\zeta) d \xi d \eta \\
& =\omega(\delta) .
\end{aligned}
$$

Isto mostra a propriedade (2.19).

Mostremos a propriedade (2.20). Para isto como $A \in C_{c}^{1}\left(\mathbb{R}^{2}\right)$, usando o Teorema da convergência dominada temos que

$$
\frac{\partial \Phi}{\partial x}=\left(f * \frac{\partial A}{\partial x}\right) \quad \text { e } \quad \frac{\partial \Phi}{\partial y}=\left(f * \frac{\partial A}{\partial y}\right)
$$

$\log \mathrm{O}$

$$
\begin{aligned}
(\bar{\partial} \Phi)(z) & =\frac{1}{2}\left(\frac{\partial \Phi(z)}{\partial x}+i \frac{\partial \Phi(z)}{\partial y}\right)=\frac{1}{2}\left[\left(f * \frac{\partial A}{\partial x}\right)(z)+i\left(f * \frac{\partial A}{\partial y}\right)(z)\right] \\
& =\left[f * \frac{1}{2}\left(\frac{\partial A}{\partial x}+i \frac{\partial A}{\partial y}\right)(z)\right]=(f *(\bar{\partial} A))(z) \\
& =\iint_{\mathbb{R}^{2}} f(z-\zeta)(\bar{\partial} A)(\zeta) d \xi d \eta \underbrace{=}_{\text {por }(2.23)} \iint_{\mathbb{R}^{2}}[f(z-\zeta)-f(z)](\bar{\partial} A)(\zeta) d \xi d \eta
\end{aligned}
$$


daí temos o seguinte

$$
\begin{aligned}
|(\bar{\partial} \Phi)(z)| & \leq \iint_{\mathbb{R}^{2}}|f(z-\zeta)-f(z) \|(\bar{\partial} A)(\zeta)| d \xi d \eta \\
& \leq \sup _{|\zeta| \leq \delta}\{|f(z-\zeta)-f(z)|\} \iint_{\mathbb{R}^{2}}|(\bar{\partial} A)(\zeta)| d \xi d \eta
\end{aligned}
$$

e usando (2.17) e (2.24) obtemos que

$$
|(\bar{\partial} \Phi)(z)| \leq \frac{2 \omega(\delta)}{\delta}
$$

Para mostrar a propriedade (2.21), observe que de (2.26) segue que $\Phi_{x}$ e $\Phi_{y}$ são contínuas. Logo $\Phi \in C_{c}^{1}\left(\mathbb{R}^{2}\right)$. Então usando o Lema 2.16 temos que

$$
\Phi(z)=-\frac{1}{\pi} \iint_{\mathbb{R}^{2}} \frac{(\bar{\partial} \Phi)(\zeta)}{\zeta-z} d \xi d \eta, \quad \operatorname{com} \zeta=\xi+i \eta
$$

Portanto, somente falta mostrar que $\bar{\partial} \Phi=0$ em $G$, onde $G=\left\{z \in K ; d\left(z, K^{c}\right)>\delta\right\}$. Para isto vamos mostrar que

$$
\Phi(z)=f(z), \quad \forall z \in G
$$

De fato, note que $\bar{\partial} f=0 \mathrm{em} G$, pois $f$ é holomorfa em $G$. Agora se $z \in G$, então $z-\zeta \in \stackrel{o}{K}$ para todo $\zeta \operatorname{com}|\zeta|<\delta$; logo, aplicando a propriedade do valor médio para $f$, obtemos que

$$
\begin{aligned}
\Phi(z) & =\iint_{\mathbb{R}^{2}} f(z-\zeta) A(\zeta) d \xi d \eta=\int_{0}^{\delta} a(r) r d r \int_{0}^{2 \pi} f\left(z-r e^{i \theta}\right) d \theta \\
& =2 \pi f(z) \int_{0}^{\delta} a(r) r d r=f(z) \iint_{\mathbb{R}^{2}} A(\zeta) d \xi d \eta=f(z) .
\end{aligned}
$$

Portanto, fica mostrada a propriedade (2.21).

Pela mesma definição de $X$ temos que $X$ é compacto e pode ser coberto por uma quantidade finita de discos abertos $D_{1}, \ldots, D_{n}$, de raio $2 \delta$, cujos centros não estão em $K$. Como $S^{2} \backslash K$ é conexo segue que, o centro de cada $D_{j}$ pode ser unido com $\infty$ por um caminho poligonal em $S^{2} \backslash K$. Logo cada $D_{j}$ contém um conjunto compacto e conexo $E_{j}$ de diâmetro pelo menos $2 \delta$, de modo que $S^{2} \backslash K$ é conexo e $K \cap E_{j}=\emptyset$.

Agora aplicando o Lema 2.15, com $r=2 \delta$, existem funções $g_{j} \in H\left(S^{2} \backslash E_{j}\right)$ e constantes 
$b_{j}$ tal que para todo $z \notin E_{j}$ e para todo $\zeta \in D_{j}$, se satisfazem as seguintes desigualdades

$$
\begin{gathered}
\left|Q_{j}(\zeta, z)\right|<\frac{50}{\delta} \\
\left|Q_{j}(\zeta, z)-\frac{1}{z-\zeta}\right|<\frac{4,000 \delta^{2}}{|z-\zeta|^{3}}
\end{gathered}
$$

onde

$$
Q_{j}(\zeta, z)=g_{j}(z)+\left(\zeta-b_{j}\right) g_{j}^{2}(z)
$$

Seja

$$
\Omega=\left(E_{1} \cup E_{2} \cup \cdots \cup E_{n}\right)^{c}=\bigcap_{j=1}^{n}\left(S^{2} \backslash E_{j}\right),
$$

então $\Omega$ é aberto e $K \subset \Omega$.

Considere agora

$$
X_{1}=X \cap D_{1} \quad \text { e } \quad X_{j}=\left(X \cap D_{j}\right) \backslash\left(X_{1} \cup X_{2} \cup \cdots \cup X_{j-1}\right), \quad \text { para } 2 \leq j \leq n,
$$

observe que $X=\bigcup_{j=1}^{n} X_{j}$. Defina

$$
R(\zeta, z)=Q_{j}(\zeta, z) \quad, \quad \text { para } \zeta \in X_{j}, z \in \Omega
$$

e

$$
F(z)=\frac{1}{\pi} \iint_{X}(\bar{\partial} \Phi)(\zeta) R(\zeta, z) d \xi d \eta \quad, \quad \text { para } z \in \Omega
$$

Como

$$
F(z)=\sum_{j=1}^{n} \frac{1}{\pi} \iint_{X_{j}}(\bar{\partial} \Phi)(\zeta) Q_{j}(\zeta, z) d \xi d \eta
$$

usando (2.29) obtemos que

$$
F(z)=\sum_{j=1}^{n} \frac{1}{\pi} \iint_{X_{j}}(\bar{\partial} \Phi)(\zeta)\left[g_{j}(z)+\left(\zeta-b_{j}\right) g_{j}^{2}(z)\right] d \xi d \eta
$$

Ou seja, $F$ é uma combinação linear finita das funções $g_{j}$ e $g_{j}^{2}$. Portanto, $F \in H(\Omega)$.

Por outro lado, de (2.21) e (2.31) temos que

$$
|F(z)-\Phi(z)| \leq \frac{1}{\pi} \iint_{X}|(\bar{\partial} \Phi)(\zeta)|\left|R(\zeta, z)-\frac{1}{\zeta-z}\right| d \xi d \eta
$$


e usando a deisgualdade (2.20) obtemos o seguinte

$$
|F(z)-\Phi(z)|<\frac{2 \omega(\delta)}{\delta} \iint_{X}\left|R(\zeta, z)-\frac{1}{\zeta-z}\right| d \xi d \eta, \quad z \in \Omega
$$

Observe que as desigualdades (2.27) e (2.28) são válidas com $R$ no lugar de $Q_{j}$ se $\zeta \in X$ e $z \in \Omega$; pois se $\zeta \in X$, então $\zeta \in X_{j}$, para algum $j$, e $\operatorname{assim} R(\zeta, z)=Q_{j}(\zeta, z)$ para todo $z \in \Omega$.

Agora fixe $z \in \Omega$ e coloque $\zeta=z+\rho e^{i \theta}$, vamos estimar a integral na equação (2.33) em duas partes.

Se $\rho<4 \delta$, neste caso usamos (2.27), então

$$
\begin{aligned}
\iint_{X}\left|R(\zeta, z)-\frac{1}{\zeta-z}\right| d \xi d \eta & \leq \iint_{X}\left(|R(\zeta, z)|+\frac{1}{|z-\zeta|}\right) d \xi d \eta \\
& <\iint_{X}\left(\frac{50}{\delta}+\frac{1}{|z-\zeta|}\right) d \xi d \eta \\
& =\int_{0}^{4 \delta} \int_{0}^{2 \pi}\left(\frac{50}{\delta}+\frac{1}{\rho}\right) \rho d \theta d \rho \\
& =2 \pi \int_{0}^{4 \delta}\left(\frac{50 \rho}{\delta}+1\right) d \rho \\
& =808 \pi \delta .
\end{aligned}
$$

Se $\rho \geq 4 \delta$, neste caso usamos (2.28), então

$$
\begin{aligned}
\iint_{X}\left|R(\zeta, z)-\frac{1}{\zeta-z}\right| d \xi d \eta & <\iint_{X} \frac{4000 \delta^{2}}{|z-\zeta|^{3}} d \xi d \eta=\int_{4 \delta}^{\infty} \int_{0}^{2 \pi} \frac{4000 \delta^{2}}{\rho^{3}} \rho d \theta d \rho \\
& =2 \pi \int_{4 \delta}^{\infty} \frac{4000 \delta^{2}}{\rho^{2}} d \rho=2000 \pi \delta
\end{aligned}
$$

Logo temos que

$$
\begin{aligned}
|F(z)-\Phi(z)| & <\frac{2 \omega(\delta)}{\pi \delta} \iint_{X}\left|R(\zeta, z)-\frac{1}{z-\zeta}\right| d \xi d \eta<\frac{2 \omega(\delta)}{\pi \delta}(808 \pi \delta+2000 \pi \delta) \\
& =5616 \omega(\delta)<6000 \omega(\delta) .
\end{aligned}
$$

Portanto,

$$
|F(z)-\Phi(z)|<6000 \omega(\delta), \quad z \in \Omega
$$

Como $F \in H(\Omega), K \subset \Omega$ e $S^{2} \backslash K$ é conexo, pelo Teorema de Runge obtemos um polinômio 
$P$ tal que

$$
|F(z)-P(z)|<\omega(\delta), \quad z \in K \text {. }
$$

Portanto, para $z \in K$, usando (2.19), (2.34) e (2.35) temos que

$$
\begin{aligned}
|f(z)-P(z)| & \leq|f(z)-\Phi(z)|+|\Phi(z)-F(z)|+|F(z)-P(z)| \\
& <6002 \omega(\delta)<10000 \omega(\delta) .
\end{aligned}
$$

Isto completa a demonstração do teorema.

\subsection{O Teorema clássico de Radó}

Agora vamos enunciar e mostrar o Teorema clássico de Radó que será usado no capítulo 5 de nosso trabalho. A demonstração é baseada no trabalho de Kaufman [8], mas pode-se consultar uma outra demonstração em [12].

Teorema 2.19. Suponha que $f \in \mathcal{C}(\bar{D}(0,1))$ e é holomorfa em $U$, onde

$$
U=\{z \in D(0,1) ; f(z) \neq 0\}
$$

Então f é holomorfa em $D(0,1)$.

Demonstração. Vamos fazer a prova deste teorema em 4 passos.

Primeiro passo. Suponha que $g \in \mathcal{C}(\bar{U})$ e holomorfa em $U$, então mostraremos que para cada $a \in U$ se satisfaz a seguinte desigualdade

$$
|g(a)| \leq \sup \{|g(z)| ; z \in \partial U,|z|=1\}
$$

De fato, observe que $U=\bigcup_{k \in \mathbb{N}} U_{k}$ onde cada $U_{k}$ é componente conexa de $U$ e além disso, $\partial U_{k} \subset \partial U$.

Também temos que para cada $n=1,2, \ldots, g^{n} f$ é holomorfa em $U$ e, portanto, em cada uma da suas componentes conexas. Então pelo princípio do máximo para cada $a \in U$ temos que

$$
\left|g^{n}(a) f(a)\right| \leq \sup \left\{\left|g^{n}(z) f(z)\right| ; z \in \partial U_{k}\right\} \leq \sup \left\{\left|g^{n}(z) f(z)\right| ; z \in \partial U\right\}
$$

Por outro lado, se $|z|<1$ e $z \in \partial U$, então existe uma sequência $z_{\epsilon} \subset D(0,1)-U$ tal que $z_{\epsilon} \rightarrow z$ e como $f$ é contínua temos que $f\left(z_{\epsilon}\right) \rightarrow f(z)$. Mas $f\left(z_{\epsilon}\right)=0$, para todo $\epsilon>0$, 
então segue que $f(z)=0$. Logo, isto e (2.37) implicam

$$
\left|g^{n}(a) f(a)\right| \leq \sup \left\{\left|g^{n}(z) f(z)\right| ; z \in \partial U,|z|=1\right\}
$$

De (2.38) temos que

$$
|g(a)|^{n}|f(a)| \leq(\sup \{|g(z)| ; z \in \partial U,|z|=1\})^{n} \cdot \sup \{|f(z)|,|z|=1\},
$$

extraindo a $n$-ésima raiz obtemos

$$
|g(a)||f(a)|^{\frac{1}{n}} \leq \sup \{|g(z)| ; z \in \partial U,|z|=1\}(\sup \{|f(z)|,|z|=1\})^{\frac{1}{n}}
$$

fazendo $n \rightarrow \infty$ na desigualdade anterior, obtemos (2.36).

Segundo passo. Vamos mostrar que $U$ é denso em $D(0,1)$. Para isto suponhamos por contradição que $U$ não é denso em $D(0,1)$.

Seja $z_{0} \in \partial U \cap D(0,1) \subset D(0,1) \cap \bar{U}$, logo existe $\left\{w_{n}\right\} \subset D(0,1)-(D(0,1) \cap \bar{U})$ tal que $w_{n} \rightarrow z_{0}$. Em seguida considere a sequência $\left\{g_{n}\right\}$, onde

$$
g_{n}(z)=\frac{1}{z-w_{n}}, z \in \bar{U}
$$

temos que $g_{n} \in \mathcal{C}(\bar{U}) \cap H(U)$ e pelo primeiro passo temos que

$$
\left|g_{n}(a)\right| \leq \sup \left\{\left|g_{n}(z)\right| ; z \in \partial U,|z|=1\right\}, \quad \forall a \in U
$$

Por outro lado se $g(z)=\frac{1}{z-z_{0}}$, temos que $g_{n}(z)$ converge para $g(z)$, para todo $z \in \stackrel{o}{U}$, e afirmamos que $\left\{g_{n}\right\}$ converge uniformemente para $g$ em $\partial U \cap S(0,1)$.

De fato, dado $\epsilon>0$, se $R=1-\left|z_{0}\right|$, existe $n_{0}$ tal que $\forall n>n_{0}\left|z-w_{n}\right|>R, \forall z \epsilon$ $\partial U \cap S(0,1)$, uma vez que $\left|z-w_{n}\right| \rightarrow\left|z-z_{0}\right|$ uniformemente em $\mathbb{C}$. Daí temos que se $n \geq n_{0}$,

$$
\begin{aligned}
\left|g_{n}(z)-g(z)\right| & =\left|\frac{1}{z-w_{n}}-\frac{1}{z-z_{0}}\right| \\
& =\frac{\left|w_{n}-z_{0}\right|}{\left|z-w_{n}\right|\left|z-z_{0}\right|} \\
& <\frac{\left|w_{n}-z_{0}\right|}{R^{2}} \\
& <\frac{\epsilon}{R^{2}}, \quad \forall z \in \partial U \cap S(0,1) .
\end{aligned}
$$


Deste fato e fazendo $n \rightarrow \infty$ em (2.39), obtemos

$$
|g(a)| \leq \sup \{|g(z)| ; z \in \partial U,|z|=1\}, \forall a \in U
$$

o qual é um absurdo pois $g$ não é limitada em $U$.

Terceiro passo. Neste passo vamos mostrar que $f$ é harmônica em $D(0,1)$. Para isto provaremos que Ref é harmônica em $D(0,1)$, e de maneira similar se mostra para $\operatorname{Imf}$. Seja $u$ solução do problema de Dirichlet seguinte

$$
\left\{\begin{array}{ccc}
\Delta u & =0 & \text { em } D(0,1) \\
u & =\operatorname{Ref} & \text { em } S(0,1)
\end{array}\right.
$$

e considere a função $H$ holomorfa em $D(0,1)$ tal que

$$
H=u+i \operatorname{Im} H
$$

Logo

$$
H=\sum_{k=0}^{+\infty} \frac{H^{k}(0)}{k !} z^{k}
$$

Assim, se fazemos $p_{n}(z)=S_{n}(z)=\sum_{k=0}^{n} \frac{H^{k}(0)}{k !} z^{k}$ temos que, existe uma sequência $\left\{p_{n}\right\}$ de polinômios tais que $\operatorname{Re}\left(p_{n}-f\right) \rightarrow 0$ uniformemente em $S(0,1)$.

Se agora, $\left|\operatorname{Re}\left(p_{n}(z)-f(z)\right)\right|<\frac{1}{n}$, para $z \in S(0,1)$, então

$$
\begin{aligned}
\left|\exp \left[p_{n}(z)-f(z)\right]\right| & =\exp \left\{\operatorname{Re}\left[p_{n}(z)-f(z)\right]\right\} \\
& \leq \exp \left|\operatorname{Re}\left[p_{n}(z)-f(z)\right]\right| \\
& <\exp \frac{1}{n}, \quad z \in S(0,1) .
\end{aligned}
$$

Analogamente temos que

$$
\left|\exp \left[f(z)-p_{n}(z)\right]\right|<\exp \frac{1}{n}, \quad z \in S(0,1) .
$$

Resumindo para todo $z \in S(0,1)$ se satisfaz o seguinte:

$$
\left|\exp \left[p_{n}(z)-f(z)\right]\right|<\exp \frac{1}{n} \text { e }\left|\exp \left[f(z)-p_{n}(z)\right]\right|<\exp \frac{1}{n}
$$


Por outro lado, aplicando (2.36) para $\exp \left[p_{n}(z)-f(z)\right]$ e $\exp \left[f(z)-p_{n}(z)\right]$ e usando $(2.40)$ obtemos para $a \in U$ que

$$
\begin{aligned}
& \left|\exp \left[p_{n}(a)-f(a)\right]\right| \leq \sup \left\{\left|\exp \left[p_{n}(z)-f(z)\right]\right| ; z \in \partial U, z \in S(0,1)\right\}<\exp \frac{1}{n} \\
& \left|\exp \left[f(a)-p_{n}(a)\right]\right| \leq \sup \left\{\left|\exp \left[f(z)-p_{n}(z)\right]\right| ; z \in \partial U, z \in S(0,1)\right\}<\exp \frac{1}{n} .
\end{aligned}
$$

Das desigualdades anteriores e pela densidade de $U$ em $D(0,1)$ concluímos que

$$
\left|\exp \left[p_{n}(z)-f(z)\right]\right|<\exp \frac{1}{n} \quad \text { e } \quad\left|\exp \left[f(z)-p_{n}(z)\right]\right|<\exp \frac{1}{n}, \forall z \in D(0,1)
$$

Temos de (2.41) que

$$
\left|R e\left(f(z)-p_{n}(z)\right)\right|<\frac{1}{n}, \forall z \in D(0,1) .
$$

Fazendo $n \rightarrow \infty$, obtemos que $\operatorname{Re}\left(p_{n}\right) \longrightarrow \operatorname{Re} f(f)$ uniformemente em $D(0,1)$ e como o limite uniforme de funçoes harmônicas é uma função harmônica, vem que $R e(f)$ é harmônica.

Quarto passo. Como $f$ é harmônica em $D(0,1)$, temos que $\bar{\partial} f$ é harmônica em $D(0,1)$ e, por hipótese, zero no subconjunto denso $U$. portanto, $f$ é holomorfa em $D(0,1)$. 


\section{Capítulo}

3

\section{Estruturas involutivas e Estruturas localmente integráveis}

Neste capítulo vamos introduzir os conceitos básicos da teoria de estruturas involutivas e localmente integráveis, bem como algumas de suas propriedades. Além disso, iremos mostrar o Teorema de geradores locais o qual nos permitirá construir coordenadas locais e geradores locais adequados para o subfibrado vetorial $T^{\prime}$ e estudaremos um dos resultados mais importantes nesta teoria o Teorema de aproximação de Baouendi-Treves. O leitor deverá possuir conhecimentos em análise real, análise complexa em uma variável, conhecimentos básicos em análise complexa em várias variáveis e equações diferenciais parciais lineares. Nossas principais referências para este capítulo são [1] e [3].

Nas duas primeiras seções vamos lembrar algumas noções padron tais como variedades diferenciáveis, campos de vetores, formas diferenciais, etc., com o objetivo principal de estabelecer a base para a apresentação e para estabelecer as notações.

\subsection{Campos vetoriais complexos sobre variedades}

Definição 3.1. Seja $\Omega$ um espaço topológico Hausdorff com base enumerável. Uma estrutura defierenciável em $\Omega$ de dimensão $N$ é uma coleção de pares

$$
\mathfrak{F}=\left\{\left(U_{\alpha}, \varphi_{\alpha}\right) ; \alpha \in \Lambda\right\}
$$

onde cada $U_{\alpha} \subset \Omega$ é aberto e $\varphi_{\alpha}: U_{\alpha} \longrightarrow \mathbb{R}^{N}$ é um homeomorfismo sobre o aberto $\varphi_{\alpha}\left(U_{\alpha}\right) \subset \mathbb{R}^{N}$.

Além disso, as seguintes propriedades devem ser satisfeitas 
i) $\bigcup_{\alpha \in \Lambda} U_{\alpha}=\Omega$

ii) $\varphi_{\alpha} \circ \varphi_{\beta}^{-1}: \varphi_{\beta}\left(U_{\alpha} \cap U_{\beta}\right) \longrightarrow \varphi_{\alpha}\left(U_{\alpha} \cap U_{\beta}\right)$ é difeomorfismo $C^{\infty}$, para todo $\alpha, \beta \in \Lambda$.

iii) Se $V \subset \Omega$ é aberto, $V \neq \emptyset$ e $\psi: V \longrightarrow \psi(V)$ é um homeomorfismo tal que, para todo $\left(U_{\alpha}, \varphi_{\alpha}\right) \in \mathfrak{F}$ com $U_{\alpha} \cap V \neq \emptyset$, temos que $\psi \circ \varphi_{\alpha}^{-1}: \varphi_{\alpha}\left(U_{\alpha} \cap V\right) \longrightarrow \psi\left(U_{\alpha} \cap V\right)$ é um difeomorfismo $C^{\infty}$, então $(V, \psi) \in \mathfrak{F}$.

Observação 3.2. Dada $\mathfrak{F}^{*}=\left\{\left(V_{\beta}, \psi_{\beta}\right) ; \beta \in \mathfrak{B}\right\}$ satisfazendo $\left.i\right)$ e ii), então existe uma única estrutura diferenciável $\mathfrak{F}$ em $\Omega$ tal que $\mathfrak{F}^{*} \subset \mathfrak{F}$.

De fato, defina $\mathfrak{F}=\{(U, \varphi) ; U \subset \Omega$ aberto , $\varphi: U \longrightarrow \varphi(U)$ homeomorfismo e $\varphi \circ \psi_{\beta}^{-1}: \psi_{\beta}\left(U_{\beta} \cap U\right) \longrightarrow \varphi\left(U_{\beta} \cap U\right)$ é difeomorfismo $\left.C^{\infty} \forall\left(U_{\beta}, \psi_{\beta}\right) \in \mathfrak{F}^{*}\right\}$.

Notemos que $\mathfrak{F}$ satisfaz $i$ ) e $i i i)$. Vejamos se $\mathfrak{F}$ satisfaz $i i)$, para isto, sejam $\left(U_{1}, \varphi_{1}\right)$, $\left(U_{2}, \varphi_{2}\right) \in \mathfrak{F}$ tais que $U_{1} \cap U_{2} \neq \emptyset$. Como $\bigcup_{\beta \in \mathfrak{B}} V_{\beta}=\Omega$, então dado $p \in U_{1} \cap U_{2}$ existe $\left(V_{\beta}, \psi_{\beta}\right) \in \mathfrak{F}^{*}$ tal que $p \in V_{\beta}$ e, portanto,

$$
\varphi_{2} \circ \varphi_{1}^{-1}=\left(\varphi_{2} \circ \psi_{\beta}^{-1}\right) \circ\left(\psi_{\beta} \circ \varphi_{1}^{-1}\right): \varphi_{1}\left(U_{1} \cap U_{2}\right) \longrightarrow \varphi_{2}\left(U_{1} \in U_{2}\right) \text { é difeomorfismo } C^{\infty} \text {, }
$$

pois por construção da $\mathfrak{F}$ temos que $\varphi_{2} \circ \psi_{\beta}^{-1}$ e $\psi_{\beta} \circ \varphi_{1}^{-1}$ são difeomorfismos $C^{\infty}$.

Definição 3.3. Uma variedade diferenciável de dimensão $N$ é um par $(\Omega, \mathfrak{F})$ onde $\Omega$ é espaço topológico Hausdorff com base enumerável e $\mathfrak{F}$ é uma estrutura diferenciável de dimensão $N$ sobre $\Omega$.

Exemplo 3.4. Seja $\Omega=\mathbb{R}^{N}$ e considere $\mathfrak{F}^{*}=\left\{\left(\mathbb{R}^{N}, I d\right)\right\}$ temos que $\left(\Omega, \mathfrak{F}^{*}\right)$ satisfaz $\left.i\right)$ e ii) pela observação anterior vem que se

$$
\mathfrak{F}=\left\{(U, \varphi) ; \varphi: U \longrightarrow \varphi(U) \text { é difeomorfismo } C^{\infty}\right\}
$$

então $(\Omega, \mathfrak{F})$ é uma variedade diferenciável.

Exemplo 3.5. Seja $(\Omega, \mathfrak{F})$ uma variedade diferenciável e $W \subset \Omega$ aberto então $W$ admite uma estrutura diferenciável "por restrições" $\left(W, \mathfrak{F}_{W}\right)$ onde

$$
\mathfrak{F}_{W}=\left\{\left(U_{\beta} \cap W,\left.\varphi_{\beta}\right|_{W}\right) ;\left(U_{\beta}, \varphi_{\beta}\right) \in \mathfrak{F}\right\}
$$

Notação: Seja $(\Omega, \mathfrak{F})$ uma variedade diferenciável de dimensão $N$, um par $(U, \varphi) \in \mathfrak{F}$ é chamado de carta ou sistema de coordenadas.

A função $\varphi$ denota-se por $\varphi=\left(x_{1}, x_{2}, \ldots, x_{N}\right)$, ou seja, $\varphi(p)=\left(x_{1}(p), x_{2}(p), \ldots, x_{N}(p)\right)$, para todo $p \in U$. 
Definição 3.6. Seja $(\Omega, \mathfrak{F})$ uma variedade diferenciável, uma função $f: \Omega \longrightarrow \mathbb{C}$ pertence $a C^{\infty}(\Omega)$ se para toda $(U, \varphi) \in \mathfrak{F}$ tem-se $f \circ \varphi^{-1}: \varphi(U) \longrightarrow \mathbb{C}$ é de classe $C^{\infty}$ em $\varphi(U)$.

Observação 3.7. O conjunto

$$
C^{\infty}(\Omega)=C^{\infty}(\Omega, \mathbb{C})=\left\{f: \Omega \longrightarrow \mathbb{C} ; f \text { é de classe } C^{\infty}\right\}
$$

é uma álgebra sobre $\mathbb{C}$, ou seja, é um espaço vetorial complexo no qual temos uma noção de produto.

Escrevemos $C^{\infty}(\Omega, \mathbb{R})$ para denotar o subconjunto de $C^{\infty}(\Omega)$ formado pelas funções $f: \Omega \longrightarrow \mathbb{C} \operatorname{com} f(\Omega) \subseteq \mathbb{R}$.

De agora em diante denotamos $\Omega$, como sendo uma variedade sem especificar a sua estrutura diferenciável.

Definição 3.8. Um campo vetorial complexo sobre $\Omega$ é uma aplicação $\mathbb{C}$-linear $L: C^{\infty}(\Omega) \longrightarrow C^{\infty}(\Omega)$, isto é:

$$
L(f+\alpha g)=L(f)+\alpha L(g), \quad \forall f, g \in C^{\infty}(\Omega), \forall \alpha \in \mathbb{C},
$$

além disso, satisfaz a seguinte regra de Leibniz

$$
L(f g)=f L(g)+g L(f), \quad \forall f, g \in C^{\infty}(\Omega) .
$$

Notação: Vamos denotar por $\mathfrak{X}(\Omega)$ o conjunto de todos os campos vetoriais complexos sobre $\Omega$, ou seja

$$
\mathfrak{X}(\Omega)=\left\{L: C^{\infty}(\Omega) \longrightarrow C^{\infty}(\Omega) ; L \text { é campo vetorial complexo }\right\}
$$

Definição 3.9. Seja $f$ uma função em $C^{\infty}(\Omega)$, o suporte da $f$ é dado por

$$
\operatorname{supp}(f)=\overline{\{p \in \Omega ; f(p) \neq 0\}} \text {. }
$$

Proposição 3.10. Seja $L \in \mathfrak{X}(\Omega)$ e $f \in C^{\infty}(\Omega)$ então, temos a seguintes propriedades:

a) Se $f$ é constante então $L f=0$.

b) $\operatorname{supp}(L f) \subseteq \operatorname{supp}(f)$.

Demonstração. 
Seja $\alpha \in \mathbb{C}$, temos que $L(\alpha)=L(\alpha .1)=\alpha L(1)$, logo para provar $a)$ basta mostrar que $L(1)=0$. Mas $L(1)=L(1.1)=1 L(1)+1 L(1)=2 L(1)$, e como $C^{\infty}(\Omega)$ é uma álgebra vem que $L(1)=0$.

Mostrar b) é equivalente mostrar que, se $V$ é aberto e $\left.f\right|_{V} \equiv 0$, então $\left.L f\right|_{V} \equiv 0$.

Suponha que $\left.f\right|_{V} \equiv 0$ e $p \in V$ arbitrário, tomemos uma carta $(U, \varphi)$ com $p \in U$ tal que $U \subset V$. Seja $\chi \in C_{c}^{\infty}(\varphi(U))$ tal que $\chi(\varphi(p))=1$, então a função $g: \Omega \longrightarrow \mathbb{C}$ definida por

$$
g(q)=\left\{\begin{array}{cl}
\chi(\varphi(q)), & q \in U \\
0, & q \notin U
\end{array}\right.
$$

pertence ao espaço $C^{\infty}(\Omega, \mathbb{R}), g \equiv 0$ em $\Omega \backslash V$ e $g(p)=1$. Como $f . g \equiv 0$ em $\Omega$ então podemos escrever

$$
f=f-0=f-f g=(1-g) f
$$

e daí temos que

$$
L(f)(p)=L((1-g) f)(p)=\underbrace{(1-g)(p)}_{0} L(f)(p)+\underbrace{f(p)}_{0} L(1-g)(p)=0,
$$

ou seja, $L(f)(p)=0$ e como $p$ é arbitrario, vem que

$$
\left.L f\right|_{V} \equiv 0 .
$$

O seguinte corolário resulta como consequência da proposição anterior.

Corolário 3.11. Se $f, g \in C^{\infty}(\Omega)$ e $f=g$ numa vizinhança de $p \in \Omega$ então $L f(p)=L g(p)$.

Demonstração. Basta aplicar o item $b$ ) da proposição anterior para a função $h=f-g$.

Observação 3.12. Como consequência do Corolário 3.11 vem que, todo campo $L \in \mathfrak{X}(\Omega)$ admite uma restrição a um aberto $W \subseteq \Omega$, ou seja, existe um elemento $L_{W} \in \mathfrak{X}(W)$ que faz o diagrama abaixo ficar comutativo

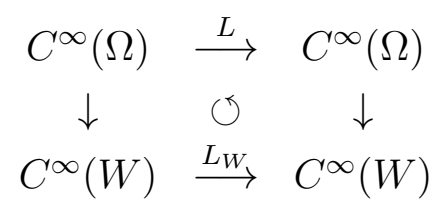

Onde as setas verticais indicam as funções restrições. 
Definição 3.13. Seja $W \subseteq \Omega$ aberto, $p \in W, f \in C^{\infty}(W)$ e $L \in \mathfrak{X}(\Omega)$ definimos $L_{W}: C^{\infty}(W) \longrightarrow C^{\infty}(W)$ por $L_{W}(f)(p)=L(\tilde{f})(p)$, onde $\tilde{f}$ é qualquer elemento de $C^{\infty}(\Omega)$ que coincide com $f$ em uma vizinhança $V$ de p. Podemos tomar $\tilde{f}=f \chi$ onde $\chi \in C_{c}^{\infty}(\Omega), \operatorname{supp}(\chi) \subset W$ e $\left.\chi\right|_{V} \equiv 1$.

Definição 3.14. Seja $L \in \mathfrak{X}(\Omega)$ e $g \in C^{\infty}(\Omega)$ definimos $g L \in \mathfrak{X}(\Omega)$ da seguinte forma

$$
g L(f)(p) \doteq g(p) L f(p)
$$

Essa multiplicação externa dá a $\mathfrak{X}(\Omega)$ uma estrutura de $C^{\infty}(\Omega)-$ mdulo.

Definição 3.15. Dados $L, M \in \mathfrak{X}(\Omega)$, definimos o comutador de $L$ e $M$ como

$$
[L, M]=L M-M L
$$

Proposição 3.16. Se $L, M \in \mathfrak{X}(\Omega)$, então $[L, M] \in \mathfrak{X}(\Omega)$.

Demonstração. Temos que provar que $[L, M]$ é linear e que satisfaz a regra de Leibniz. De fato, sejam $f, g \in C^{\infty}(\Omega)$ e $\alpha \in \mathbb{C}$.

i) Linearidade:

$$
\begin{aligned}
{[L, M](f+\alpha g) } & =L M(f+\alpha g)-M L(f+\alpha g) \\
& =L(M(f)+\alpha M(g))-M(L(f)+\alpha L(g)) \\
& =L(M f)+\alpha L(M g)-M(L f)-\alpha M(L g) \\
& =L(M f)-M(L f)+\alpha(L(M g)-M(L g)) \\
& =(L M-M L)(f)+\alpha(L M-M L)(g) \\
& =[L, M](f)+\alpha[L, M](g) .
\end{aligned}
$$

ii) Regra de Leibniz:

$$
\begin{aligned}
{[L, M](f . g)=} & (L M-M L)(f . g) \\
= & L M(f . g)-M L(f . g) \\
= & L(f M g+g M f)-M(f L g+g L f) \\
= & L(f M g)+L(g M f)-M(f L g)-M(g L f) \\
= & f L(M g)+M g L f+g L(M f)+M f L g-f M(L g)- \\
& -L g M f-g M(L f)-L f M g \\
= & f(L(M g)-M(L g))+g(L(M f)-M(L f)) \\
= & f[L, M](g)+g[L, M](f) .
\end{aligned}
$$


Agora seja $(U, \varphi)$ uma carta em $\Omega$ e $f \in C^{\infty}(U)$ e $\varphi(q)=\left(x_{1}(q), \ldots, x_{N}(q)\right)$. Considere o diagrama seguinte

$$
\begin{aligned}
& C^{\infty}(U) \longrightarrow C^{\infty}(\varphi(U)) \longrightarrow C^{\infty}(\varphi(U)) \longrightarrow C^{\infty}(U) \\
& f \quad \longmapsto \quad f \circ \varphi^{-1} \longmapsto \frac{\partial}{\partial x_{j}}\left(f \circ \varphi^{-1}\right) \longmapsto h \circ \varphi
\end{aligned}
$$

onde $h=\frac{\partial}{\partial x_{j}}\left(f \circ \varphi^{-1}\right)$.

Definição 3.17. Dada $(U, \varphi)$ uma carta em $\Omega$ e $f \in C^{\infty}(U)$, definimos

$$
\frac{\partial f}{\partial x_{j}} \doteq\left\{\frac{\partial}{\partial x_{j}}\left(f \circ \varphi^{-1}\right)\right\} \circ \varphi, \quad j=1,2 \ldots, N .
$$

Proposição 3.18. Seja $(U, \varphi)$ uma carta local em $\Omega$, então $\frac{\partial}{\partial x_{j}} \in \mathfrak{X}(U)$.

Demonstração. Sejam $f, g \in C^{\infty}(U)$ e $\alpha \in \mathbb{C}$.

i) Linearidade:

$$
\begin{aligned}
\frac{\partial}{\partial x_{j}}(f+\alpha g) & \doteq\left\{\frac{\partial}{\partial x_{j}}\left((f+\alpha g) \circ \varphi^{-1}\right)\right\} \circ \varphi \\
& =\left\{\frac{\partial}{\partial x_{j}}\left(f \circ \varphi^{-1}+\alpha g \circ \varphi^{-1}\right)\right\} \circ \varphi \\
& =\left\{\frac{\partial}{\partial x_{j}}\left(f \circ \varphi^{-1}\right)+\alpha \frac{\partial}{\partial x_{j}}\left(g \circ \varphi^{-1}\right)\right\} \circ \varphi \\
& =\left\{\frac{\partial}{\partial x_{j}}\left(f \circ \varphi^{-1}\right)\right\} \circ \varphi+\alpha\left\{\frac{\partial}{\partial x_{j}}\left(g \circ \varphi^{-1}\right)\right\} \circ \varphi \\
& =\frac{\partial f}{\partial x_{j}}+\alpha \frac{\partial g}{\partial x_{j}}
\end{aligned}
$$

ii) Regra de Leibniz:

$$
\begin{aligned}
\frac{\partial}{\partial x_{j}}(f . g) & \doteq\left\{\frac{\partial}{\partial x_{j}}\left((f . g) \circ \varphi^{-1}\right)\right\} \circ \varphi \\
& =\left\{\frac{\partial}{\partial x_{j}}\left(f \circ \varphi^{-1} . g \circ \varphi^{-1}\right)\right\} \circ \varphi \\
& =\left\{f \circ \varphi^{-1} \frac{\partial}{\partial x_{j}}\left(g \circ \varphi^{-1}\right)+g \circ \varphi^{-1} \frac{\partial}{\partial x_{j}}\left(f \circ \varphi^{-1}\right)\right\} \circ \varphi \\
& =f\left\{\frac{\partial}{\partial x_{j}}\left(g \circ \varphi^{-1}\right)\right\} \circ \varphi+g\left\{\frac{\partial}{\partial x_{j}}\left(f \circ \varphi^{-1}\right)\right\} \circ \varphi \\
& =f \frac{\partial g}{\partial x_{j}}+g \frac{\partial f}{\partial x_{j}}
\end{aligned}
$$


Teorema 3.19. Seja $L \in \mathfrak{X}(\Omega)$ e $(U, \varphi)$ carta local em $\Omega, \varphi=\left(x_{1}, \ldots, x_{N}\right)$, então

a) $L_{U}=\sum_{j=1}^{N}\left(L x_{j}\right) \frac{\partial}{\partial x_{j}}$.

b) $\left\{\frac{\partial}{\partial x_{1}}, \ldots, \frac{\partial}{\partial x_{N}}\right\}$ é base para o $C^{\infty}(U)$-módulo $\mathfrak{X}(U)$.

Demonstração. Vamos mostrar o item a). Para isto seja $p \in U$ e $V \subset U$ aberto , $p \in V$ tal que $\varphi(V)=B(a, r)$ onde $a=\varphi(p)$ e seja $f \in C^{\infty}(U)$.

Se $q \in V$ então $\varphi(q)=\left(x_{1}(q), \ldots, x_{N}(q)\right) \in B(a, r)$, agora na bola $B(a, r)$ usamos a fórmula de Taylor com resto integral.

$$
\left(f \circ \varphi^{-1}\right)(x)=\left(f \circ \varphi^{-1}\right)(a)+\sum_{j=1}^{N} h_{j}(x)\left(x_{j}-a_{j}\right)
$$

onde cada $h_{j} \in C^{\infty}(B(a, r))$ é dada por $h_{j}(x)=\int_{0}^{1} \frac{\partial}{\partial x_{j}}\left(f \circ \varphi^{-1}\right)(a+t(x-a)) d t$ para todo $x \in B(a, r)$.

Agora em $V$ temos

$$
f(q)=f(p)+\sum_{j=1}^{N}\left(h_{j} \circ \varphi\right)(q)\left(x_{j}-x_{j}(p)\right)(q)
$$

aplicando $L$ à expressão anterior temos

$$
\begin{aligned}
L_{V}(f)(q) & =L_{V}(f(p))+\sum_{j=1}^{N} L_{V}\left[\left(h_{j} \circ \varphi\right)(q)\left(x_{j}-x_{j}(p)\right)(q)\right] \\
& =\sum_{j=1}^{N}\left(h_{j} \circ \varphi\right)(q) L_{V}\left(x_{j}(q)-x_{j}(p)\right)+\left(x_{j}(q)-x_{j}(p)\right) L_{V}\left(h_{j} \circ \varphi\right)(q) \\
& =\sum_{j=1}^{N}\left(h_{j} \circ \varphi\right)(q) L_{V}\left(x_{j}\right)(q)+\left(x_{j}(q)-x_{j}(p)\right) L_{V}\left(h_{j} \circ \varphi\right)(q) .
\end{aligned}
$$

Tomando $p=q$ segue que

$$
\begin{aligned}
L_{V}(f)(q) & =\sum_{j=1}^{N}\left(h_{j} \circ \varphi\right)(p) L_{V}\left(x_{j}\right)(p)=\sum_{j=1}^{N} L_{V}\left(x_{j}\right)(p) h_{j}(\varphi(p)) \\
& =\sum_{j=1}^{N} L_{V}\left(x_{j}\right)(p) \frac{\partial\left(f \circ \varphi^{-1}\right)}{\partial x_{j}}(a)=\sum_{j=1}^{N} L_{V}\left(x_{j}\right)(p) \frac{\partial}{\partial x_{j}}\left(f \circ \varphi^{-1}\right) \circ \varphi(p)
\end{aligned}
$$


daí vem que

$$
L_{U}=\sum_{j=1}^{N} L\left(x_{j}\right) \frac{\partial}{\partial x_{j}}
$$

Como consequência do item a) temos que $\left\{\frac{\partial}{\partial x_{1}}, \ldots, \frac{\partial}{\partial x_{N}}\right\}$ gera $\mathfrak{X}(U)$. Logo para mostrarmos o item $b$ ) é suficiente provar que este conjunto é linearmente independente. De fato, sejam $g_{1}, g_{2}, \ldots, g_{N} \in C^{\infty}(U)$ tais que $L=\sum_{j=1}^{N} g_{j} \frac{\partial}{\partial x_{j}}=0$.

Considere as funções $f_{k}=\rho_{k} \circ \varphi$ onde $\rho_{k}: \mathbb{R}^{n} \longrightarrow \mathbb{R}, \rho_{k}(x)=x_{k}$, temos que $f_{k} \in C^{\infty}(U)$ e

$$
\frac{\partial f_{k}}{\partial x_{j}} \doteq\left\{\frac{\partial}{\partial x_{j}}\left(f_{k} \circ \varphi^{-1}\right)\right\} \circ \varphi=\left\{\frac{\partial}{\partial x_{j}}\left(\rho_{k} \circ \varphi \circ \varphi^{-1}\right)\right\} \circ \varphi=\frac{\partial \rho_{k}}{\partial x_{j}} \circ \varphi=\delta_{k j}
$$

assim para cada $1 \leq k \leq N$, temos

$$
0=L f_{k}=g_{1} \frac{\partial f_{k}}{\partial x_{1}}+\cdots+g_{k} \frac{\partial f_{k}}{\partial x_{k}}+\cdots+g_{N} \frac{\partial f_{k}}{\partial x_{n}}=g_{k} .
$$

Observação 3.20. Seja $(U, \varphi)$ uma carta local em $\Omega$ e $L, M \in \mathfrak{X}(U)$ então a representação de $[L, M]$ em coordenadas locais é dado por

$$
\begin{aligned}
{\left.[L, M]\right|_{U} } & =\sum_{j=1}^{N}\left(L\left(M x_{j}\right)-M\left(L x_{j}\right)\right) \frac{\partial}{\partial x_{j}} \\
& =\sum_{j=1}^{N}[L, M]\left(x_{j}\right) \frac{\partial}{\partial x_{j}} .
\end{aligned}
$$

Definição 3.21. Se $L \in \mathfrak{X}(\Omega)$, definimos o campo $\bar{L}$ conjugado complexo de $L$ da seguinte maneira $\bar{L}(f)=\overline{L(\bar{f})}$, para toda $f \in C^{\infty}(\Omega)$.

Verifiquemos se $\bar{L}$ é um campo vetorial complexo, para isto sejam $f, g \in C^{\infty}(\Omega), \alpha \in \mathbb{C}$ mostremos primeiro que $\bar{L}$ é linear

$$
\begin{aligned}
\bar{L}(f+\alpha g) & =\overline{L(\overline{f+\alpha g})}=\overline{L(\bar{f}+\bar{\alpha} \bar{g})}=\overline{L(\bar{f})+\bar{\alpha} L(\bar{g})} \\
& =\overline{L(\bar{f})}+\alpha \overline{L(\bar{g})}=\bar{L}(f)+\alpha \bar{L}(g) .
\end{aligned}
$$


Mostremos agora que $\bar{L}$ satisfaz a regra de Leibniz

$$
\begin{aligned}
\bar{L}(f . g) & =\overline{L(\overline{f \cdot g})}=\overline{L(\bar{f} \cdot \bar{g})}=\overline{\bar{f} L(\bar{g})+\bar{g} L(\bar{f})}=\overline{\bar{f} L(\bar{g})}+\overline{\bar{g} L(\bar{f})} \\
& =f \overline{L(\bar{g})}+g \overline{L(\bar{f})}=f \bar{L}(g)+g \bar{L}(f) .
\end{aligned}
$$

Portanto, $\bar{L}$ é um campo vetorial complexo.

\section{Observação 3.22 .}

1) Notemos que $\overline{\frac{\partial}{\partial x_{j}}}=\frac{\partial}{\partial x_{j}}$. De fato, considere $f=u+i v$, logo

$$
\begin{aligned}
\overline{\frac{\partial}{\partial x_{j}}(f)} & =\overline{\frac{\partial}{\partial x_{j}}(\bar{f})}=\overline{\frac{\partial}{\partial x_{j}}(u-i v)} ;=\overline{\frac{\partial}{\partial x_{j}}(u)-i \frac{\partial}{\partial x_{j}}(v)} \\
& =\frac{\partial}{\partial x_{j}}(u)+i \frac{\partial}{\partial x_{j}}(v)=\frac{\partial}{\partial x_{j}}(u+i v)=\frac{\partial}{\partial x_{j}}(f) .
\end{aligned}
$$

2) $S e(U, \varphi)$ é um sistema de coordenadas em $\Omega \operatorname{com} \varphi=\left(x_{1}, \ldots, x_{N}\right)$, se sabe que neste sistema $L$ é dado por $L=\sum_{j=1}^{N} a_{j}(x) \frac{\partial}{\partial x_{j}}$, então

$$
\bar{L}=\overline{\sum_{j=1}^{N} a_{j}(x) \frac{\partial}{\partial x_{j}}}=\sum_{j=1}^{N} \overline{a_{j}(x)} \frac{\bar{\partial}}{\partial x_{j}}=\sum_{j=1}^{N} \overline{a_{j}(x)} \frac{\partial}{\partial x_{j}} .
$$

\subsection{Vetores tangentes complexos e 1-formas diferenciáveis}

Seja $p \in \Omega$ considere o conjunto

$$
\beta_{p}=\left\{(V, f) ; V \subset \Omega \text { aberto }, p \in V \text { e } f \in C^{\infty}(V)\right\}
$$

Em $\beta_{p}$ definimos a seguinte relação de equivalência: dados $\left(V_{1}, f_{1}\right),\left(V_{2}, f_{2}\right) \in \beta_{p}$ dizemos que $\left(V_{1}, f_{1}\right) \sim\left(V_{2}, f_{2}\right)$ se existe uma vizinhanaça aberta $V \subset V_{1} \cap V_{2} \operatorname{com} p \in V$ tal que $\left.f_{1}\right|_{V}=\left.f_{2}\right|_{V}$.

Definição 3.23. Um germe de uma função $C^{\infty}$ no ponto p é uma classe de equivalência em $\beta_{p}$, ou seja é um elemento do espaço quociente $C^{\infty}(p)=\beta_{p} / \sim$.

Se $f$ é uma função $C^{\infty}$ numa vizinhança de $p$, denotaremos o seu germe por $\underline{f}$. Pode-se mostrar que $C^{\infty}(p)$ é uma $\mathbb{C}$-álgebra. 
Observação 3.24. A aplicação

$$
\begin{array}{ccc}
T: C^{\infty}(p) & \longrightarrow & \mathbb{C} \\
\underline{f} & \longmapsto & T(\underline{f})=f(p)
\end{array}
$$

está bem definida e é um homomorfismo de $\mathbb{C}$-álgebras.

De fato, se $\underline{f}=\underline{g}$ então existe uma vizinhança aberta $V \subset \Omega, p \in V$ tal que $\left.f\right|_{V}=\left.g\right|_{V}$, então $f(p)=g(p)$, ou seja $T(\underline{f})=T(\underline{g})$. Portanto, $T$ está bem definida.

Por outro lado se $f, g \in C^{\infty}$ numa vizinhança de $p$ e $\alpha \in \mathbb{C}$ temos

$$
\begin{aligned}
& T(\underline{f} \cdot \underline{g})=T(\underline{f . g})=(f \cdot g)(p)=f(p) g(p)=T(\underline{f}) T(\underline{g}) \\
& T(\alpha \underline{f})=T(\underline{\alpha f})=(\alpha f)(p)=\alpha f(p)=(\underline{f}) \\
& T(\underline{f}+\underline{g})=T(\underline{f+g})=(f+g)(p)=f(p)+g(p)=T(\underline{f})+T(\underline{g}) .
\end{aligned}
$$

Logo $T$ é um homomorfismo de $\mathbb{C}$-álgebras.

Definição 3.25. Um vetor tangente complexo a $\Omega$ no ponto $p$ é uma aplicação

$$
v: C^{\infty}(p) \longrightarrow \mathbb{C}
$$

que é $\mathbb{C}$-linear e satisfaz a seguinte condição

$$
v(\underline{f} . \underline{g})=f(p) v(\underline{g})+g(p) v(\underline{f}), \text { para todo } \underline{f}, \underline{g} \in C^{\infty}(p) \text {. }
$$

O espaço tangente complexo a $\Omega$ em $p$ é o espaço vetorial complexo

$$
\mathbb{C} T_{p} \Omega=\{v ; v \text { é vetor tangente complexo a } \Omega \text { em } p\} \text {. }
$$

Observação 3.26. Se $L \in \mathfrak{X}(\Omega)$ e $p \in \Omega$, então definimos um elemento $L_{p} \in \mathbb{C} T_{p} \Omega d a$ seguinte forma

$$
\begin{aligned}
L_{p}: C^{\infty}(p) & \longrightarrow \mathbb{C} \\
\underline{f} & \longmapsto L_{p}(\underline{f}) \doteq(L f)(p) .
\end{aligned}
$$

$L_{p}$ está bem definida pois pelo Corolário 3.11 temos que se $\left.f\right|_{V}=\left.g\right|_{V}$, então $L f=L g$ em $V$, ou seja, se $\underline{f}=\underline{g}$, então existe uma vizinhança aberta $V$ com $p \in V$ tal que $\left.f\right|_{V}=\left.g\right|_{V}, \operatorname{logo} L_{p}(\underline{f})=L_{p}(\underline{g})$.

Mostraremos agora que $L_{p}$ é linear e que satisfaz a regra de Leibniz. De fato, sejam 
$\underline{f}, \underline{g} \in C^{\infty}(p)$ e $\alpha \in \mathbb{C}$, temos

$$
\begin{aligned}
L_{p}(\underline{f}+\alpha \underline{g}) & =L_{p}(\underline{f+\alpha g})=L(f+\alpha g)(p)=(L f+\alpha L g)(p) \\
& =L f(p)+\alpha L g(p)=L_{p}(\underline{f})+\alpha L_{p}(\underline{g}) .
\end{aligned}
$$

Por outro lado temos

$$
\begin{aligned}
L_{p}(\underline{f} \cdot \underline{g}) & =L_{p}(\underline{f . g})=L(f . g)(p)=(f L g+g L f)(p)=(f L g)(p)+(g L f)(p) \\
& =f(p) L g(p)+g(p) L f(p)=f(p) L_{p}(\underline{g})+g(p) L_{p}(\underline{f}) .
\end{aligned}
$$

Portanto $L_{p} \in \mathbb{C} T_{p} \Omega$.

Observação 3.27. Dado $\underline{f} \in C^{\infty}(p)$, pode ocorrer que $f$ não esteja definida na variedade toda. Contornamos este problema do seguinte modo.

Se $f \in C^{\infty}(V)$, com $p \in V$, tome $K \subset V$ compacto, $p \in K$ e considere a função $\chi \in C^{\infty}(\Omega)$ tal que

$$
\chi(q)= \begin{cases}1, & \text { se } q \in K \\ 0, & \text { se } q \notin V\end{cases}
$$

e faça $g=\chi f \in C^{\infty}(\Omega)$, então $\left.g\right|_{K}=\left.f\right|_{K}$ e $\left.g\right|_{V^{c}} \equiv 0$, logo temos que $\underline{g}=\underline{f}$ e defina $L_{p}(\underline{f})=(L g)(p)$.

Proposição 3.28. Seja $\Omega$ uma variedade diferenciável $e(U, \varphi)$ uma carta local, $p \in U$ e $v \in \mathbb{C} T_{p} \Omega$, então

i) $v(\underline{f})=\left.\sum_{j=1}^{N} v\left(\underline{x}_{j}\right) \frac{\partial}{\partial x_{j}}\right|_{p}(\underline{f})$, para toda $\underline{f} \in C^{\infty}(p)$.

ii) $\left\{\left.\frac{\partial}{\partial x_{1}}\right|_{p}, \ldots,\left.\frac{\partial}{\partial x_{N}}\right|_{p}\right\}$ é base de $\mathbb{C} T_{p} \Omega$.

Demonstração. Seja $\underline{f} \in C^{\infty}(p)$ e $V \subset U$ aberto $p \in V$ tal que

$$
f \in C^{\infty}(V), \quad \varphi(V)=B(a, r) \quad \text { e } \quad a=\varphi(p) .
$$

Dado $q \in V$ temos que $x=\varphi(q) \in B(a, r)$ e pela fórmula de Taylor com resto integral temos

$$
\left(f \circ \varphi^{-1}\right)(x)=\left(f \circ \varphi^{-1}\right)(a)+\sum_{j-1}^{N} h_{j}(x)\left(x_{j}-a_{j}\right), \quad \forall x \in B(a, r)
$$


onde $h_{j} \in C^{\infty}(B(a, r))$ e $h_{j}(a)=\frac{\partial}{\partial x_{j}}\left(f \circ \varphi^{-1}\right)(a)$.

Agora em $V$ temos que

$$
f(q)=f(p)+\sum_{j=1}^{N} g_{j}(q)\left(x_{j}(q)-x_{j}(p)\right), \quad \text { para qualquer } q \in V
$$

onde $g_{j}(p)=\frac{\partial}{\partial x_{j}} f(p)$, portanto

$$
\underline{f}=\underline{f}(p)+\sum_{j=1}^{N} \underline{g}_{j}\left(\underline{x}_{j}-\underline{x}_{j}(p)\right)
$$

Notemos que $\underline{f}(p)$ e $\underline{x}_{j}(p)$ são germes de funções constantes e vemos que

$$
v(\underline{c})=v(c . \underline{1})=c v(\underline{1})
$$

mas $v(\underline{1})=v(\underline{1} \cdot \underline{1})=1 v(\underline{1})+1 v(\underline{1})=2 v(\underline{1})$ o qual implica que $v(\underline{1})=0$, logo

$$
\begin{aligned}
v \underline{f} \underline{f}) & =v(\underline{f}(p))+\sum_{j=1}^{N} v\left(\underline{g}_{j}\left(\underline{x}_{j}-\underline{x}_{j}(p)\right)\right) \\
& =\sum_{j=1}^{N}\left[v\left(\underline{g}_{j} \underline{x}_{j}\right)-v\left(\underline{g}_{j} \underline{x}_{j}(p)\right)\right] \\
& =\sum_{j=1}^{N}\left[g_{j}(p) v\left(\underline{x}_{j}\right)+x_{j}(p) v\left(\underline{g}_{j}\right)-g_{j}(p) v\left(\underline{x}_{j}(p)\right)-x_{j}(p) v\left(\underline{g}_{j}\right)\right] \\
& =\sum_{j=1}^{N} g_{j}(p) v\left(\underline{x}_{j}\right) .
\end{aligned}
$$

Por outro lado temos

$$
\begin{aligned}
g_{j}(p) & =\left(h_{j} \circ \varphi\right)(p)=h_{j}(a)=\frac{\partial}{\partial x_{j}}\left(f \circ \varphi^{-1}\right)(a) \\
& =\left(\left\{\frac{\partial}{\partial x_{j}}\left(f \circ \varphi^{-1}\right)\right\} \circ \varphi\right)(p)=\frac{\partial f}{\partial x_{j}}(p)=\left.\frac{\partial}{\partial x_{j}}\right|_{p}(\underline{f}),
\end{aligned}
$$

de onde obtemos

$$
v(\underline{f})=\left.\sum_{j=1}^{N} \frac{\partial}{\partial x_{j}}\right|_{p}(\underline{f}) v\left(\underline{x}_{j}\right), \quad \text { para toda } \underline{f} \in C^{\infty}(p) .
$$

O que mostra o item $i$ ). 
Para mostrar $i i)$ basta mostrar que os vetores $\left.\frac{\partial}{\partial x_{1}}\right|_{p}, \ldots,\left.\frac{\partial}{\partial x_{N}}\right|_{p}$ são linearmente independentes, pois pelo item $i$ ) eles geram $\mathbb{C} T_{p} \Omega$. De fato, sejam $\alpha_{1}, \ldots, \alpha_{N} \in \mathbb{C}$ tais que $\left.\sum_{j=1}^{N} \alpha_{j} \frac{\partial}{\partial x_{j}}\right|_{p}=0$. Em seguida considere funções $h_{1}, \ldots, h_{N} \in C^{\infty}(U)$ tais que

$$
\frac{\partial h_{k}}{\partial x_{j}}(p)= \begin{cases}0, & \text { se } j \neq k \\ 1, & \text { se } j=k\end{cases}
$$

Então

$$
0=\left.\sum_{j=1}^{N} \alpha_{j} \frac{\partial}{\partial x_{j}}\right|_{p}\left(h_{k}\right)=\sum_{j=1}^{N} \alpha_{j} \frac{\partial h_{k}}{\partial x_{j}}(p)=\alpha_{k}
$$

para $k=1,2, \ldots, N$, portanto $\left\{\left.\frac{\partial}{\partial x_{1}}\right|_{p}, \ldots,\left.\frac{\partial}{\partial x_{N}}\right|_{p}\right\}$ é base de $\mathbb{C} T_{p} \Omega$.

Exemplo 3.29. Dado $v \in \mathbb{C} T_{p} \Omega$, existe $L \in \mathfrak{X}(\Omega)$ tal que $L_{p}=v$.

De fato, pela Proposição 3.28 temos que, dado $v \in \mathbb{C} T_{p} \Omega$ e $(U, \varphi)$ carta local com $p \in U$, podemos escrever

$$
v=\left.\sum_{j=1}^{N} v\left(\underline{x}_{j}\right) \frac{\partial}{\partial x_{j}}\right|_{p}
$$

Por outro lado, como $\left\{\left.\frac{\partial}{\partial x_{1}}\right|_{q}, \ldots,\left.\frac{\partial}{\partial x_{N}}\right|_{q}\right\}$ é base de $\mathbb{C} T_{p} \Omega$ para todo $q \in U$, então definimos o vetor $v_{q} \in \mathbb{C} T_{p} \Omega$ pondo

$$
\left.v_{q} \doteq \sum_{j=1}^{N} v\left(\underline{x}_{j}\right) \frac{\partial}{\partial x_{j}}\right|_{q}
$$

Agora seja $V \subset U$ aberto, $p \in V, K \subset V$ compacto e $g \in C^{\infty}(U)$ tal que $\left.g\right|_{K} \equiv 1$ e $\left.g\right|_{U \backslash V} \equiv 0$ e em seguida defina $L \in \mathfrak{X}(\Omega)$ tal que no sistema de coordenadas $(U, \varphi)$ em torno de $p$ é da forma

$$
L=\sum_{j=1}^{N} g v\left(\underline{x}_{j}\right) \frac{\partial}{\partial x_{j}} .
$$

Assim, $L$ está bem definido, é linear, respeita a regra de Leibniz e além disso,

$$
\begin{aligned}
L_{p}(\underline{f}) & =(L f)(p)=\sum_{j=1}^{N} g(p) v\left(\underline{x}_{j}\right) \frac{\partial}{\partial x_{j}} f(p) \\
& =\left.\sum_{j=1}^{N} v\left(\underline{x}_{j}\right) \frac{\partial}{\partial x_{j}}\right|_{p}(\underline{f})=v(\underline{f}) .
\end{aligned}
$$


Exemplo 3.30. Suponha que para cada $p \in \Omega$ é dado $v_{p} \in \mathbb{C} T_{p} \Omega$ tal que a aplicação

$$
\begin{aligned}
& \Omega \quad \mathbb{C} \\
& p \quad \longmapsto \quad v_{p}(\underline{f})
\end{aligned}
$$

é $C^{\infty}$, para todo $f \in C^{\infty}(\Omega)$ fixado. Então existe um único campo $L \in \mathfrak{X}(\Omega)$ tal que $L_{p}=v_{p}, \forall p \in \Omega$.

De fato, defina $L: C^{\infty}(\Omega) \longrightarrow C^{\infty}(\Omega)$ por $(L f)(p)=v_{p}(\underline{f})$.

Por hipótese, se $f \in C^{\infty}$ então $L f \in C^{\infty}$, além disso, se $f, g \in C^{\infty}(\Omega)$ e $\alpha \in \mathbb{C}$, então

$$
\begin{aligned}
L(\alpha f+g)(p) & =v_{p}(\underline{\alpha f+g})=v_{p}(\underline{\alpha} \underline{f}+\underline{g})=v_{p}(\underline{\alpha} \underline{f})+v_{p}(\underline{g}) \\
& =\alpha v_{p}(\underline{f})+f(p) v_{p}(\underline{\alpha})+v_{p}(\underline{g})=\alpha(L f)(p)+(L g)(p)
\end{aligned}
$$

segue que L é linear. Por outro lado,

$$
\begin{aligned}
L(f . g)(p) & =v_{p}(\underline{f . g})=v_{p}(\underline{f} . \underline{g})=f(p) v_{p}(\underline{g})+g(p) v_{p}(\underline{f}) \\
& =f(p) L f(p)+g(p) L g(p)=(f L g)+g L f)(p)
\end{aligned}
$$

satisfazendo também a regra de Leibniz. Para mostrar a unicidade, suponha que exista $M \in \mathfrak{X}(\Omega)$ tal que $M_{p}=v_{p}$, para todo $p \in \Omega$, então $M_{p}(\underline{f}) \doteq M f(p)$, para toda $\underline{f} \in C^{\infty}(p)$, daí

$$
M f(p)=M_{p}(\underline{f})=v_{p}(\underline{f})=L f(p)
$$

o que implica que $L=M$.

Definição 3.31. O fibrado tangente complexo é definido e denotado da seguinte forma

$$
\mathbb{C} T \Omega=\bigcup_{p \in \Omega} \mathbb{C} T_{p} \Omega
$$

Onde a união acima é disjunta.

Definição 3.32. Se $v \in \mathbb{C} T_{p} \Omega$ definimos $\bar{v} \in \mathbb{C} T_{p} \Omega$ (o conjugado de $v$ ) como $\bar{v}(\underline{f})=v(\underline{\bar{f}})$, para toda $\underline{f} \in C^{\infty}(p)$.

Definição 3.33. Denotemos por $\eta(\Omega)$ o dual do $C^{\infty}(\Omega)$ - módulo $\mathfrak{X}(\Omega)$, ou seja, $\omega \in \eta(\Omega)$, se $\omega: \mathfrak{X}(\Omega) \longrightarrow C^{\infty}(\Omega)$ e satisfaz

$$
\omega(f L+M)=f \omega(L)+\omega(M), \quad \forall f \in C^{\infty}(\Omega), \forall L, M \in \mathfrak{X}(\Omega) .
$$


Os elementos de $\eta(\Omega)$ são chamados de 1-formas diferenciáveis.

Lema 3.34. Se $\omega \in \eta(\Omega), L \in \mathfrak{X}(\Omega)$ e $\left.L\right|_{V} \equiv 0$, com $V \subseteq \Omega$ aberto, então $\left.\omega(L)\right|_{V} \equiv 0$. Demonstração. Dado $p \in V$, tomemos $g \in C_{c}^{\infty}(\Omega, \mathbb{R})$ tal que $g(p)=1$ e $\left.g\right|_{\Omega \backslash V} \equiv 0$. Então see $q \in V$, tem-se $L_{q}=0$ e se $q \in \Omega \backslash V$ tem-se $g(q)=0$. Logo $(g L)_{q}=g(q) L_{q}=0$, para todo $q \in \Omega$, portanto $g L=0$. Daí vem que $L=L-g L=(1-g) L$, logo

$$
\omega(L)(p)=\omega((1-g) L)(p)=(1-g)(p) \omega(L)(p)=(1-g(p)) \omega L(p)=0
$$

Portanto $\left.\omega(L)\right|_{V} \equiv 0$.

Observação 3.35. Sempre é possivel restringir um elemento de $\eta(\Omega)$ a um elemento de $\eta(W) \operatorname{com} W \subseteq \Omega$ aberto.

De fato, se $L \in \mathfrak{X}(W), p \in W$ então dada uma 1-forma $\omega \in \eta(\Omega)$ definimos

$$
\left.\omega\right|_{W}(L)(p) \doteq \omega(\tilde{L})(p)
$$

onde $\tilde{L} \in \mathfrak{X}(\Omega)$ é tal que $\tilde{L}=L$ em uma vizinhança de $p$.

Notemos que $\left.\omega\right|_{W}$ está bem definida, pois se $\tilde{L}, \tilde{M} \in \mathfrak{X}(\Omega)$ são tais que $\tilde{L}=L$ em $V_{1} \subseteq \Omega$ com $p \in V_{1}$ e $\tilde{M}=L$ em $V_{2} \subseteq \Omega$ com $p \in V_{2}, V_{1}$ e $V_{2}$ abertos, então $p \in V_{1} \cap V_{2}$ e temos que

$$
\omega(\tilde{L})(p)=\left.\omega\right|_{W}(L)(p)=\omega(\tilde{M})(p) .
$$

Além disso, a definição de $\left.\omega\right|_{W}$ não depende de $\tilde{L}$ ja que se $\tilde{L}, \tilde{M} \in \mathfrak{X}(\Omega)$ e $\tilde{M}=\tilde{L}$ em $V, p \in V$ então $\tilde{M}-\tilde{L}=0$ em $V$ e do Lema 3.34 vem que

$$
\omega(\tilde{M}-\tilde{L})(p)=0
$$

ou seja,

$$
\omega(\tilde{M})(p)=\omega(\tilde{L})(p) .
$$

Lema 3.36. Seja $\omega \in \eta(\Omega)$ e $L \in \mathfrak{X}(\Omega)$. Se $L_{p}=0$ então $\omega(L)(p)=0$.

Demonstração. Seja $(U, \varphi)$ uma carta local de $\Omega$ tal que $p \in U$ e $\varphi=\left(x_{1}, \ldots, x_{N}\right)$, então

$$
\begin{aligned}
\omega(L)(p) & =\omega_{U}\left(L_{U}\right)(p)=\omega_{U}\left(\sum_{j=1}^{N}\left(L x_{j}\right) \frac{\partial}{\partial x_{j}}\right)(p)=\left(\sum_{j=1}^{N}\left(L x_{j}\right) \omega_{U}\left(\frac{\partial}{\partial x_{j}}\right)\right)(p) \\
& =\sum_{j=1}^{N}\left(L_{U} x_{j}\right)(p) \omega_{U}\left(\frac{\partial}{\partial x_{j}}\right)(p)=\sum_{j=1}^{N} L_{p}\left(\underline{x}_{j}\right) \omega_{U}\left(\frac{\partial}{\partial x_{j}}\right)(p)=0 .
\end{aligned}
$$


Portanto $\omega(L)(p)=0$.

Definição 3.37. O dual de $\mathbb{C} T_{p} \Omega, p \in \Omega$ é denotado por $\mathbb{C} T_{P}^{*} \Omega$ e chamado de espaço cotangente a $\Omega$ em $p$.

Observação 3.38. Dada $\omega \in \eta(\Omega)$ e $p \in \Omega$ podemos definir $\omega_{p} \in \mathbb{C} T_{p}^{*} \Omega$

$$
\omega_{p}: \mathbb{C} T_{p} \Omega \longrightarrow \mathbb{C}
$$

por $\omega_{p}(v)=\omega(L)(p)$, onde $L \in \mathfrak{X}(\Omega)$ e $L_{p}=v$.

De fato, para verificar que $\omega_{p}$ está bem definida considere $L, M \in \mathfrak{X}(\Omega)$ tais que $L_{p}=v=M_{p}$, então $L_{p}-M_{p}=0$ ou equivalentemente $(L-M)_{p}=0$ pelo lema anterior temos

$$
0=\omega(L-M)(p)=\omega(L)(p)-\omega(M)(p)
$$

dai vem que

$$
\omega(L)(p)=\omega(M)(p) .
$$

Mostremos agora que $\omega_{p}$ é linear. Para isso sejam $v_{1}, v_{2} \in \mathbb{C} T_{p} \Omega, \alpha \in \mathbb{C}$ com $L_{p}=v_{1}$, $M_{p}=v_{2}$ temos que $\alpha v_{1}+v_{2}=\alpha L_{p}+M_{p}=(\alpha L+M)_{p}$. Dai

$$
\begin{aligned}
\omega_{p}\left(\alpha v_{1}+v_{2}\right) & =\omega(\alpha L+M)(p)=(\alpha \omega(L)+\omega(M))(p)=\alpha \omega(L)(p)+\omega(M)(p) \\
& =\alpha \omega_{p}(L)+\omega_{p}(M)=\alpha \omega_{p}\left(v_{1}\right)+\omega_{p}\left(v_{2}\right) .
\end{aligned}
$$

Proposição 3.39. Seja $\Omega$ uma variedade diferenciável de dimensão $N$, então

$$
\mathbb{C} T_{p}^{*} \Omega=\left\{\omega_{p} ; \omega \in \eta(\Omega)\right\}
$$

Demonstração. Pela observação anterior temos que

$$
\left\{\omega_{p} ; \omega \in \eta(\Omega)\right\} \subset \mathbb{C} T_{p}^{*} \Omega
$$

Além disso, se $(U, \varphi)$ é uma carta local com $p \in U, \varphi=\left(x_{1}, \ldots, x_{N}\right)$ e $L \in \mathfrak{X}(\Omega)$, sabemos que $L_{U}=\sum_{j=1}^{N}\left(L x_{j}\right) \frac{\partial}{\partial x_{j}}$.

Definimos $d x_{j} \in \eta(U), j=1, \ldots, N$ por $d x_{j}\left(\frac{\partial}{\partial x_{k}}\right)=\delta_{j k}$, ou seja,

$$
d x_{j}\left(L_{U}\right)=\sum_{k=1}^{N}\left(L x_{k}\right) d x_{j}\left(\frac{\partial}{\partial x_{k}}\right)=L x_{j} .
$$


Se $\omega \in \eta(U)$, então

$$
\begin{aligned}
\omega(L) & =\omega\left(\sum_{k=1}^{N}\left(L x_{k}\right) \frac{\partial}{\partial x_{k}}\right)=\sum_{k=1}^{N}\left(L x_{k}\right) \omega\left(\frac{\partial}{\partial x_{k}}\right)=\sum_{k=1}^{N} \omega\left(\frac{\partial}{\partial x_{k}}\right) L\left(x_{k}\right) \\
& =\sum_{k=1}^{N} \omega\left(\frac{\partial}{\partial x_{k}}\right) d x_{k}(L)=\left(\sum_{k=1}^{N} \omega\left(\frac{\partial}{\partial x_{k}}\right) d x_{k}\right)(L) .
\end{aligned}
$$

Portanto obtemos que

$$
\omega=\sum_{k=1}^{N} \omega\left(\frac{\partial}{\partial x_{k}}\right) d x_{k}
$$

Resumindo temos que $\left\{d x_{1_{p}}, \ldots, d x_{N_{p}}\right\}$ é base dual de $\left\{\left.\frac{\partial}{\partial x_{1}}\right|_{p}, \ldots,\left.\frac{\partial}{\partial x_{N}}\right|_{p}\right\}$ e qualquer elemento da forma $\omega_{p}$ se escreve como $\omega_{p}=\sum_{k=1}^{N} \omega\left(\frac{\partial}{\partial x_{k}}\right) d x_{k p}$.

Mostremos agora que $\mathbb{C} T_{p}^{*} \Omega \subset\left\{\omega_{p} ; \omega \in \eta(\Omega)\right\}$. De fato, se $v^{*} \in \mathbb{C} T_{p}^{*} \Omega$, então existem escalares $\alpha_{1}, \ldots, \alpha_{N}$ tais que $v^{*}=\sum_{k=1}^{N} \alpha_{k} d x_{k p}$. Agora, se $\omega=\sum_{k=1}^{N} \alpha_{k} d x_{k} \in \eta(\Omega)$ então $\omega_{p}=v^{*}$, ou seja, $v^{*} \in\left\{\omega_{p} ; \omega \in \eta(\Omega)\right\}$.

Definição 3.40. (diferencial de uma função)

Seja $f \in C^{\infty}(\Omega)$, definimos $d f \in \eta(\Omega) d f: \mathfrak{X}(\Omega) \longrightarrow C^{\infty}$ como df $(L)(p) \doteq(L f)(p)$.

Observação 3.41. Notemos que se $g \in C^{\infty}$ e $L, M \in \mathfrak{X}(\Omega)$ temos que

$$
\begin{aligned}
d f(g L+M)(p) & =(g L+M)(f)(p) \\
& =g(p)(L f)(p)+(M f)(p) \\
& =g(p) d f(L)(p)+d f(M)(p) .
\end{aligned}
$$

Mais ainda, se $(U, \varphi)$ é carta local de $\Omega$ com $p \in U$ e $\varphi=\left(x_{1}, \ldots, x_{N}\right)$ temos que

$$
\begin{aligned}
d f(L)(p) & =(L f)(p)=\sum_{j=1}^{N}\left(L x_{j}\right)(p) \frac{\partial f}{\partial x_{j}}(p)=\sum_{j=1}^{N} d x_{j}(L)(p) \frac{\partial f}{\partial x_{j}}(p) \\
& =\left(\sum_{j=1}^{N} d x_{j}(L) \frac{\partial f}{\partial x_{j}}\right)(p)
\end{aligned}
$$

Portanto

$$
d f=\sum_{j=1}^{N} \frac{\partial f}{\partial x_{j}} d x_{j}
$$


Definição 3.42. O fibrado cotangente complexo é definido e denotado por

$$
\mathbb{C} T^{*} \Omega=\bigcup_{p \in \Omega} \mathbb{C} T_{p}^{*} \Omega
$$

onde a união acima é disjunta.

Observação 3.43. Seja $\Omega$ uma variedade diferenciável de dimensão $N$, temos as seguintes notações.

$$
\begin{aligned}
& T_{p} \Omega=\left\{v \in \mathbb{C} T_{p} \Omega ; v \text { é real }\right\} \\
& T_{p}^{*} \Omega=\left\{\xi \in \mathbb{C} T_{p}^{*} \Omega ; \xi \text { é real }\right\} \\
& T \Omega=\bigcup_{p \in \Omega} T_{p} \Omega, \quad T^{*}=\bigcup_{p \in \Omega} T_{p}^{*} \Omega
\end{aligned}
$$

\subsection{Estruturas localmente integráveis}

Nesta parte do trabalho vamos definir o que é uma estrutura involutiva e uma estrutura localmente integrável, mas para isso precisamos da noção de subfibrado vetorial complexo que é o conteúdo da seguinte definição. Salvo menção explícita em contrário, ao longo desta seção, $\Omega$ representará uma variedade diferenciável suave de dimensão $N$.

Definição 3.44. Um subfibrado vetorial complexo de $\mathbb{C} T \Omega$ de dimensão $n$, com $1 \leq n \leq$ $N$ é da forma $\mathcal{V}=\bigcup_{p \in \Omega} \mathcal{V}_{p}$ satisfazendo as seguintes condições:

i) $C a d a \mathcal{V}_{p} \subseteq \mathbb{C} T_{p} \Omega$ é um subespaço vetorial de dimensão $n$.

ii) Para cada $p_{0} \in \Omega$ existe um aberto $U_{0} \subset \Omega, p_{0} \in U_{0}$ e campos $L_{1}, \ldots, L_{n} \in \mathfrak{X}\left(U_{0}\right)$ tais que $L_{1 q}, \ldots, L_{n q}$ geram $\mathcal{V}_{q}$, para todo $q \in U_{0}$.

Definição 3.45. Uma seção do subfibrado vetorial complexo $\mathcal{V}$ sobre o aberto $W \subset \Omega$ é um campo vetorial $L \in \mathfrak{X}(W)$ tal que $L_{p} \in \mathcal{V}_{p}$, para todo $p \in W$.

Definição 3.46. Uma estrutura involutiva (ou formalmente integrável) é um subfibrado vetorial complexo $\mathcal{V} \subseteq \mathbb{C} T \Omega$ tal que dado qualquer $W \subset \Omega$ aberto e seções $L, M \in \mathfrak{X}(W)$ de $\mathcal{V}$ sobre $W$, então $[L, M]$ é também uma seção de $\mathcal{V}$ sobre $W$.

Em outras palavras, $[L, M]$ também é combinação linear de $L_{1 p}, \ldots, L_{n p}$ em $\mathcal{V}_{p}$.

Exemplo 3.47. Seja $L \in \mathfrak{X}(\Omega)$ um campo vetorial complexo não singular, ou seja, $L_{p} \neq 0$, para todo $p \in \Omega$. Então $\mathcal{V}=<L>=\left\{f L ; f \in C^{\infty}(\Omega)\right\}$ é uma estrutura involutiva. 
De fato, primeiro verifiquemos que $\mathcal{V}$ é subfibrado, se $\mathcal{V}_{p}=\left\{\alpha L_{p} ; \alpha \in \mathbb{C}\right\}$ teremos

$$
\mathcal{V}=<L>=\left\{f L ; f \in C^{\infty}(\Omega)\right\}=\bigcup_{p \in \Omega}\left\{f(p) L_{p} ; f \in C^{\infty}(\Omega)\right\}=\bigcup_{p \in \Omega} \mathcal{V}_{p}
$$

onde $\mathcal{V}_{p} \subseteq \mathbb{C} T_{p} \Omega$ é subespaço vetorial complexo de dimensão 1 gerado pelo vetor $L_{p}$. Observe ainda que $f L$ é uma seção de $\mathcal{V}$ sobre qualquer aberto $W \subset \Omega$ para qualquer $f \in C^{\infty}(\Omega)$, pois $f(p) L_{p} \in \mathcal{V}_{p}$, para todo $p \in \Omega$.

Verifiquemos agora que $\mathcal{V}$ é uma estrutura involutiva, para isso, consideremos um aberto $W \subset \Omega$ e duas seções $f L, g L \in \mathfrak{X}(W)$ de $\mathcal{V}$ sobre $W$ com $f, g \in C^{\infty}(\Omega)$ temos que

$$
\begin{aligned}
{[f L, g L] } & =f L(g L)-g L(f L) \\
& =f(g L L+L(g) L)-g(f L L+L(f) L) \\
& =f g L L+f L(g) L-g f L L-g L(f) L \\
& =(f L(g)-g L(f)) L
\end{aligned}
$$

então

$$
\left.[f L, g L]\right|_{p}=(f(p) L g(p)-g(p) L f(p)) L_{p} \in \mathcal{V}_{p}
$$

Daí vem que $\mathcal{V}$ é uma estrutura involutiva.

Observação 3.48. Seja $\mathcal{V}$ uma estrutura involutiva sobre $\Omega$, pela definição de subfibrado vetorial obtemos uma carta $(U, \varphi)$ contendo $p$ e campos $L_{1}, \ldots, L_{n} \in \mathfrak{X}(U)$ tais que os vetores $L_{1 q}, \ldots, L_{n_{q}}$ formam uma base para $\mathcal{V}_{q}$ para todo $q \in U$. Se $\varphi=\left(x_{1}, \ldots, x_{N}\right)$ então

$$
L_{j}=\sum_{k=1}^{N} a_{j k} \frac{\partial}{\partial x_{k}} \quad, \quad j=1, \ldots, n
$$

onde $a_{j k}=L_{j} x_{k} \in C^{\infty}(U)$.

Como $\mathcal{V}$ é uma estrutura involutiva temos que $\left.\left[L_{j}, L_{l}\right]\right|_{p} \in \mathcal{V}_{p}, 1 \leq j, l \leq n$.

Exemplo 3.49. Na observação acima, pode-se mostrar que existem funções $C_{j l}^{\nu} \in C^{\infty}(U)$ tais que

$$
\left[L_{j}, L_{l}\right]=\sum_{\nu=1}^{n} C_{j l}^{\nu} L_{\nu} .
$$

De fato, na própria definição de involutividade temos a existência das funções $C_{j l}^{\nu}$, mais ainda é possivel mostrar que estas funções são de classe $C^{\infty}$ em $U$. 
De forma análoga, podemos também introduzir a noção de subfibrado vetorial complexo do fibrado cotangente complexo $\mathbb{C} T^{*} \Omega$.

Definição 3.50. Um subfibrado vetorial complexo de $\mathbb{C} T^{*} \Omega$ de dimensão $m$ é da forma $\mathcal{W}=\bigcup_{p \in \Omega} \mathcal{W}_{p}$ satisfazendo a seguintes condições:

i) Cada $\mathcal{W}_{p}$ é subespaço vetorial de $C T_{p}^{*} \Omega$ com dimensão fixa $m, 0 \leq m \leq N$.

ii) Para cada $p_{0} \in \Omega$ existe um aberto $U_{0} \subset \Omega$ com $p_{0} \in U_{0}$ e existem, 1-formas $\omega_{1}, \ldots, \omega_{m}$ tais que $\omega_{1 p}, \ldots, \omega_{m p}$ geram $\mathcal{W}_{q}$ para todo $q \in U_{0}$.

Proposição 3.51. Seja $\mathcal{V}=\bigcup_{p \in \Omega} \mathcal{V}_{p}$ um subfibrado vetorial de $\mathbb{C} T \Omega$, então

$$
\mathcal{V}^{\perp}=\bigcup_{p \in \Omega} \mathcal{V}_{p}^{\perp}
$$

é um subfibrado vetorial de $\mathbb{C} T^{*} \Omega$, onde

$$
\mathcal{V}_{p}^{\perp}=\left\{\lambda \in \mathbb{C} T_{p}^{*} \Omega ; \lambda(v)=0, \forall v \in \mathcal{V}_{p}\right\}
$$

Demonstração. Temos $m=\operatorname{dim}_{p}^{\perp}=N-n$ onde $n=\operatorname{dim} \mathcal{V}_{p}$.

Por outro lado como $\mathcal{V}$ é um subfibrado, dado $p \in \Omega$ tem-se $(U, \varphi)$ carta local de $\Omega, p \in U, \varphi=\left(x_{1}, \ldots, x_{N}\right)$ e campos $L_{1}, \ldots, L_{n} \in \mathfrak{X}(U)$ tais que $\left\{L_{1 q}, \ldots, L_{n_{q}}\right\}$ é base de $\mathcal{V}_{q}$, para todo $q \in U$. Além disso, sabemos que $L_{j}=\sum_{k=1}^{N} a_{j k} \frac{\partial}{\partial x_{k}}, j=1, \ldots, n$ onde $a_{j k} \in C^{\infty}(U)$.

Como, os campos $L_{1}, \ldots, L_{n}$ são linearmente independentes em $U$, então em particular no ponto $p$ a matriz $\left(a_{j k}(p)\right)_{n \times N}$ tem uma submatriz $n \times n$ com determinante diferente de zero.

Fazendo uma permutação nas variáveis $x_{1}, \ldots, x_{N}$ (caso seja necessário) podemos supor que $\operatorname{det}\left\{\left(a_{j k}(p)\right), 1 \leq j, k \leq n\right\} \neq 0$, logo pela continuidade da função determinante e regularidade das funções $\left(a_{j k}\right), 1 \leq j \leq n$ obtemos uma vizinhança de $p$, a qual iremos continuar denotando por $U$, na qual $\operatorname{det}\left\{\left(a_{j k}(q)\right), 1 \leq j, k \leq n\right\} \neq 0$, para todo $q \in U$. Agora seja $\left(b_{j k}\right)_{1 \leq j, k \leq n}$ a matriz inversa de $\left(a_{j k}\right)_{1 \leq j, k \leq n}$ em $U$ e considere os seguintes campos vetoriais

$$
L_{j}^{\sharp}=\sum_{\nu=1}^{n} b_{j \nu} L_{\nu} d, \quad j=1, \ldots, n .
$$

Então os vetores $L_{1 q}^{\sharp}, \ldots, L_{n q}^{\sharp}$ também formam uma base para $\mathcal{V}_{q}$, para todo $q \in U$, além 
disso,

$$
\begin{aligned}
L_{j}^{\sharp} & =\sum_{\nu=1}^{n} b_{j \nu} L_{\nu}=\sum_{\nu=1}^{n} b_{j \nu}\left(\sum_{k=1}^{N} a_{\nu k} \frac{\partial}{\partial x_{k}}\right) \\
& =\sum_{\nu=1}^{n} b_{j \nu}\left(\sum_{k=1}^{n} a_{\nu k} \frac{\partial}{\partial x_{k}}+\sum_{k=n+1}^{N} a_{\nu k} \frac{\partial}{\partial x_{k}}\right) \\
& =\sum_{\nu=1}^{n} \sum_{k=1}^{n} b_{j \nu} a_{\nu k} \frac{\partial}{\partial x_{k}}+\sum_{\nu=1}^{n} \sum_{k=n+1}^{N} b_{j \nu} a_{\nu k} \frac{\partial}{\partial x_{k}} \\
& =\sum_{k=1}^{n}\left(\sum_{\nu=1}^{n} b_{j \nu} a_{\nu k}\right) \frac{\partial}{\partial x_{k}}+\sum_{k=n+1}^{n}\left(\sum_{\nu=1}^{n} b_{j \nu} a_{\nu k}\right) \frac{\partial}{\partial x_{k}} \\
& =\sum_{k=1}^{n}\left(\sum_{\nu=1}^{n} b_{j \nu} a_{\nu k}\right) \frac{\partial}{\partial x_{k}}+\sum_{k=1}^{m}\left(\sum_{\nu=1}^{n} b_{j \nu} a_{\nu k}\right) \frac{\partial}{\partial x_{n+k}} .
\end{aligned}
$$

Note que $\sum_{\nu=1}^{n} b_{j \nu} a_{\nu k}$ é $(j, k)$-ésimo elemento da matriz $\left(b_{j k}\right)\left(a_{j k}\right)=I d$, logo vem que

$$
L_{j}^{\sharp}=\frac{\partial}{\partial x_{j}}+\sum_{k=1}^{m} c_{j k} \frac{\partial}{\partial x_{n+k}}, \quad j=1, \ldots, n,
$$

onde $c_{j k}=\sum_{\nu=1}^{n} b_{j \nu} a_{\nu k} \in C^{\infty}(U)$.

Agora que estabelecemos uma expresão conveniente para uma base de $\mathcal{V}_{q}^{\perp}$, definimos

$$
\omega_{l}=d x_{n+l}-\sum_{\gamma=1}^{n} c_{\gamma l} d x_{\gamma}, \quad l=1, \ldots, m
$$

Observe que $\left\{\omega_{1 q}, \ldots, \omega_{m q}\right\}$ é um conjunto linearmente independente em cada $q \in U$ e

$$
\begin{aligned}
\omega_{l}\left(L_{j}^{\sharp}\right) & =\left(d x_{n+l}-\sum_{\gamma=1}^{n} c_{\gamma l} d x_{\gamma}\right)\left(\frac{\partial}{\partial x_{j}}+\sum_{k=1}^{m} c_{j k} \frac{\partial}{\partial x_{n+k}}\right) \\
& =d x_{n+l}\left(\frac{\partial}{\partial x_{j}}\right)+d x_{n+l}\left(\sum_{k=1}^{m} c_{j k} \frac{\partial}{\partial x_{n+k}}\right)-\sum_{\gamma=1}^{n} c_{\gamma l} d x_{\gamma}\left(\frac{\partial}{\partial x_{j}}\right)-\sum_{\gamma=1}^{n} c_{\gamma l} d x_{\gamma}\left(\sum_{k=1}^{m} c_{j k} \frac{\partial}{\partial x_{n+k}}\right) \\
& =\sum_{k=1}^{m} c_{j k} d x_{n+l}\left(\frac{\partial}{\partial x_{n+k}}\right)-\sum_{\gamma=1}^{n} c_{\gamma l} d x_{\gamma}\left(\frac{\partial}{\partial x_{j}}\right)-\sum_{\gamma=1}^{n} \sum_{k=1}^{m} c_{\gamma l} c_{j k} d x_{\gamma}\left(\frac{\partial}{\partial x_{n+k}}\right) \\
& =\sum_{k=1}^{m} c_{j k} \delta_{(n+l, n+k)}-\sum_{\gamma=1}^{n} c_{\gamma l} \delta_{\gamma j} \\
& =c_{j l}-c_{j l}=0 .
\end{aligned}
$$

Portanto $\omega_{l} \in \mathcal{V}_{q}^{\perp}$, para todo $q \in U$. 
Isto mostra que em cada ponto $q \in U$ o ortogonal $\mathcal{V}_{q}^{\perp}$ é um espaço vetorial gerado pelos vetores cotangentes $\left\{\omega_{1 q}, \ldots, \omega_{m q}\right\}$, de onde vem que $\mathcal{V}^{\perp}=\bigcup_{p \in \Omega} \mathcal{V}_{p}^{\perp}$ é um subfibrado de $\mathbb{C} T^{*} \Omega$.

Exemplo 3.52. Se $\mathcal{V}=<\frac{\partial}{\partial x_{1}}, \ldots, \frac{\partial}{\partial x_{n}}>$, então $\mathcal{V}^{\perp}=<d x_{n+1}, \ldots, x_{n+m}>\operatorname{com} n+m=$ $N$.

Notação: Se $\mathcal{V}$ for uma estrutura involutiva sobre $\Omega$ denotamos $\mathcal{V}^{\perp}$ por $T^{\prime}$ e sempre $\operatorname{dim} \mathcal{V}=n, \operatorname{dim} T^{\prime}=m$ e $n+m=N$.

Vamos dar agora a definição de conjunto característico de uma estrutura involutiva e do símbolo de um campo vetorial, para isto, considere $\mathcal{V} \subseteq \mathbb{C} T \Omega$ uma estrutura involutiva e $T^{\prime}=\mathcal{V}^{\perp}$ o subfibrado ortogonal.

Definição 3.53. O conjunto característico de $\mathcal{V}$ é o conjunto

$$
T^{0}=T^{\prime} \cap T^{*} \Omega
$$

onde $T^{*} \Omega$ é o fibrado cotangente real dado como na Observação 3.43.

Definição 3.54. O símbolo $\sigma(L)$ do campo vetorial $L \in \mathfrak{X}(\Omega)$ é a aplicação $\sigma(L): T^{*} \Omega \longrightarrow \mathbb{C}$ definida por $\sigma(L)(p, \xi)=\xi\left(L_{p}\right)$, para todo $\xi \in T_{p}^{*} \Omega$.

Agora dado um sistema de coordenadas $(U, \varphi)$ em torno de $p$ com $\varphi=\left(x_{1}, \ldots, x_{N}\right)$ e $\xi \in T_{p}^{*} \Omega$, podemos escrever

$$
\xi=\left.\sum_{k=1}^{N} \xi_{k} d x_{k}\right|_{p}
$$

$\operatorname{com} \xi_{j} \in \mathbb{R}$ e $L=\sum_{j=1}^{N} a_{j}(x) \frac{\partial}{\partial x_{j}}, a_{j} \in C^{\infty}(U)$, então

$$
\sigma(L)(p, \xi)=\xi\left(L_{p}\right)=\left.\sum_{k=1}^{N} \sum_{j=1}^{N} \xi_{k} a_{j}(p) d x_{k}\right|_{p}\left(\left.\frac{\partial}{\partial x_{j}}\right|_{p}\right)=\sum_{j=1}^{N} a_{j}(p) \xi_{j}
$$

Observação 3.55. Seja $\mathcal{V}=<L>$ o subfibrado gerado por um único campo nãa singular $L \in \mathfrak{X}(U)$. Vimos que $\mathcal{V}$ é involutiva, neste caso $\mathcal{V}=<L>=\left\{f L ; f \in C^{\infty}(U)\right\}$, assim $\mathcal{V}_{p}=\left\{f(p) L_{p} ; f \in C^{\infty}(U)\right\}=\left\{\alpha L_{p} ; \alpha \in \mathbb{C}\right\}$. Desta maneira, se $\lambda \in \mathcal{V}_{p}^{\perp}$, então $0=\lambda\left(\alpha L_{p}\right)=\alpha \lambda\left(L_{p}\right)$, para todo $\alpha \in \mathbb{C}$, ou seja $\mathcal{V}_{p}^{\perp}=\left\{\lambda \in \mathbb{C} T_{p}^{*} \Omega ; \lambda\left(L_{p}\right)=0\right\}$.

Supondo que $(U, \varphi)$ é um sistema de coodenadas centradas em $p, \operatorname{com} \varphi=\left(x_{1}, \ldots, x_{N}\right)$ 
e que $\lambda=\left.\sum_{k=1}^{N} \xi_{k} d x_{k}\right|_{p}, \xi_{k} \in \mathbb{C}$ e $L=\sum_{j=1}^{N} a_{j}(x) \frac{\partial}{\partial x_{j}}, a_{j} \in C^{\infty}(U)$, temos

$$
\begin{aligned}
\mathcal{V}_{p}^{\perp} & =\left\{\lambda \in \mathbb{C} T_{p}^{*} \Omega ; \sum_{j=1}^{N} a_{j}(p) \xi_{j}=0, \xi_{j} \in \mathbb{C}\right\} \\
& =\left\{\left(\xi_{1}, \ldots, \xi_{N}\right) \in \mathbb{C}^{N} ; \sum_{j=1}^{N} a_{j}(p) \xi_{j}=0\right\} .
\end{aligned}
$$

Daí interceptando $T^{\prime}=\bigcup_{p \in \Omega} \mathcal{V}_{p}^{\perp}$ com o fibrado tangente real $T^{*} \Omega=\bigcup_{p \in \Omega} T_{p}^{*} \Omega$ teremos

$$
T^{0}=\left\{(p, \xi) ; \sum_{j=1}^{N} a_{j}(p) \xi_{j}=0, \xi_{j} \in \mathbb{R}\right\}
$$

$\mathrm{Ou}$

$$
\begin{aligned}
T^{0} & =\left\{\left(\xi_{1}, \ldots, \xi_{N}\right) \in \mathbb{R}^{N} ; \sum_{j=1}^{N} a_{j}(p) \xi_{j}=0\right\} \\
& =\left\{\xi \in \mathbb{R}^{N} ; \sigma(L)(p, \xi)=0\right\} .
\end{aligned}
$$

Portanto, para determinar o conjunto característico de um operador $L$, ou seja, para determinar o conjunto característico de uma estrutura involutiva $\mathcal{V}$ gerado por um único campo $L$ precisamos saber em quais dos pontos de $\mathbb{R}^{N}$ o símbolo se anula.

Exemplo 3.56. Se $L=\sum_{j=1}^{N} a_{j}(x) \frac{\partial}{\partial x_{j}}, a_{j} \in C^{\infty}(\Omega, \mathbb{R})$ e $N \geq 2$, então $T^{0}$ é não trivial.

De fato, o símbolo de $L$ é $\sigma(L)(x, \xi)=\sum_{j=1}^{N} a_{j}(x) \xi_{j} \operatorname{com} a_{j} \in C^{\infty}(U, \mathbb{R}), \log \mathrm{o} \sigma(L)(x, \xi)=0$ $\operatorname{com} \xi_{1}, \ldots, \xi_{N} \in \mathbb{R}$ não todos nulos.

Exemplo 3.57. Se $\frac{\partial}{\partial \bar{z}}=\frac{1}{2}\left(\frac{\partial}{\partial x}+i \frac{\partial}{\partial y}\right) \in \mathfrak{X}\left(\mathbb{R}_{(x, y)}^{2}\right)$, então $T^{0}=\{(0,0)\}$.

De fato, $\sigma\left(\frac{\partial}{\partial \bar{z}}\right)\left(x, y, \xi_{1}, \xi_{2}\right)=\frac{1}{2}\left(\xi_{1}+i \xi_{2}\right)=0$ se e somente se $\xi_{1}=\xi_{2}=0$, por tanto

$$
T^{0}=\{(0,0)\}
$$

Definição 3.58. Se $\mathcal{V}=<L_{1}, \ldots, L_{n}>$, onde $L_{j}=\sum_{k=1}^{N} a_{j k} \frac{\partial}{\partial x_{k}}, j=1, \ldots, N$ em $(U, \varphi)$, então o conjunto característico de $\mathcal{V}$ sobre $U$ é descrito pelas equações

$$
\sum_{k=1}^{N} a_{j k}(p) \xi_{k}=0
$$


comp $p \in U, \xi_{k} \in \mathbb{R}$ e $j=1, \ldots, N$.

Exemplo 3.59. O operador de Mizohata $M=\frac{\partial}{\partial t}-i t \frac{\partial}{\partial x} \in \mathfrak{X}\left(\mathbb{R}_{(x, t)}^{2}\right), \xi=\xi_{1} d x+\xi_{2} d t$, temos que $\sigma(M)\left(x, t, \xi_{1}, \xi_{2}\right)=\xi_{2}-i t \xi_{1}=0$ se e somente se $\xi_{2}=0$ ou $t \xi_{1}=0$, por tanto

$$
T_{(x, t)}^{0}= \begin{cases}\{(0,0)\} & , \text { se } t \neq 0 \\ \left\{\left.\xi_{1} d x\right|_{p} ; \xi_{1} \in \mathbb{R}\right\} & , \text { se } t=0\end{cases}
$$

em particular temos que

$$
\operatorname{dim} T_{(x, t)}^{0}= \begin{cases}0, & \text { se } t \neq 0 \\ 1, & \text { se } t=0\end{cases}
$$

$\operatorname{logo} T^{0}$ não é subfibrado.

Definição 3.60. Se $\mathcal{V}=\bigcup_{p \in \Omega} \mathcal{V}_{p} \subseteq \mathbb{C} T \Omega$ é um subfibrado vetorial, definimos o subfibrado conjugado de $\mathcal{V}$ por

$$
\overline{\mathcal{V}}=\bigcup_{p \in \Omega} \overline{\mathcal{V}}_{p}
$$

onde $\overline{\mathcal{V}}_{p}=\left\{\bar{v} ; v \in \mathcal{V}_{p}\right\}$

Observação 3.61. Uma definição análoga pode-se dar para um subfibrado vetorial de $\mathbb{C} T^{*} \Omega$, mais ainda, a seguinte igualdade é satisfeita

$$
(\overline{\mathcal{V}})^{\perp}=\overline{\left(\mathcal{V}^{\perp}\right)}
$$

Daremos agora algumas estruturas especiais que pode-se encontrar na teoria das estrururas involutivas.

Definição 3.62. Seja $\mathcal{V} \subseteq \mathbb{C} T \Omega$ uma estrutura involutiva. Então $\mathcal{V}$ é dita uma estrutura

i) Elitica se $T_{p}^{0}=\{0\}$, para todo $p \in \Omega$.

ii) Complexa se $\mathcal{V}_{p} \oplus \overline{\mathcal{V}_{p}}=\mathbb{C} T_{p} \Omega$, para todo $p \in \Omega$.

iii) $C$ - $R$ se $\mathcal{V}_{p} \cap \overline{\mathcal{V}_{p}}=0$, para todo $p \in \Omega$.

iv) Essencialmente real se $\mathcal{V}_{p}=\overline{\mathcal{V}_{p}}$, para todo $p \in \Omega$.

Exemplo 3.63. seja $\frac{\partial}{\partial \bar{z}}=\frac{1}{2}\left(\frac{\partial}{\partial x}+i \frac{\partial}{\partial y}\right) \in \mathfrak{X}\left(\mathbb{R}_{(x, y)}^{2}\right)$ e $\mathcal{V}=<\frac{\partial}{\partial \bar{z}}>$, então $\mathcal{V}$ é estrutura C-R e elítica. 
De fato, já vimos que $T_{(x, y)}^{0}=\{(0,0)\}$, para todo $(x, y) \in \mathbb{R}^{2}$, então $\mathcal{V}$ é elítica. Agora se $v \in \mathcal{V}_{p} \cap \overline{\mathcal{V}_{p}}$, então $v=\left.\alpha \frac{\partial}{\partial \bar{z}}\right|_{p}$ e $v=\left.\beta \frac{\partial}{\partial z}\right|_{p}$, com $\alpha, \beta \in \mathbb{C}$, assim

$$
\left.\alpha \frac{\partial}{\partial \bar{z}}\right|_{p}=\left.\beta \frac{\partial}{\partial z}\right|_{p} \quad \Longleftrightarrow \quad \alpha=\beta=0
$$

o qual implica que $v=0$, portanto $\mathcal{V}$ é C-R.

Exemplo 3.64. Seja $\frac{\partial}{\partial x_{j}} \in \mathfrak{X}\left(\mathbb{R}^{N}\right)$, então $\mathcal{V}=<\frac{\partial}{\partial x_{j}}>$ é essencialmente real.

De fato, observamos anteriormente que $\frac{\bar{\partial}}{\partial x_{j}}=\frac{\partial}{\partial x_{j}}$, assim

$$
\begin{gathered}
\overline{\mathcal{V}_{p}}=\left\{\bar{v} ; v \in \mathcal{V}_{p}\right\}=\left\{\bar{v} ; v=\alpha \frac{\partial}{\partial x_{j}}, \alpha \in \mathbb{C}\right\}=\left\{\left.\bar{\alpha} \frac{\partial}{\partial x_{j}}\right|_{p} ; \alpha \in \mathbb{C}\right\} \\
=\left\{\left.\beta \frac{\partial}{\partial x_{j}}\right|_{p} ; \beta \in \mathbb{C}\right\}=\mathcal{V}_{p} \\
\therefore \mathcal{V}_{p}=\overline{\mathcal{V}}_{p}, \text { para todo } p \in \mathbb{R}^{N} .
\end{gathered}
$$

Isto é $\mathcal{V}$ é uma estrutura essencialmente real.

Na seguinte definição vamos introduzir o que vem a ser um dos conceitos mais importantes da teoria de estruturas involutivas e, portanto, também para este trabalho.

Definição 3.65. (Estrutura localmente integrável).

Seja $\mathcal{V}$ uma estrutura involutiva sobre $\Omega$, dizemos que $\mathcal{V}$ é localmente integrável se para cada $p \in \Omega$, existe um aberto $U \subseteq \Omega$ com $p \in U$ e funções $Z_{1}, \ldots, Z_{m} \in C^{\infty}(\Omega)$ tais que $\left\{d Z_{1}(q), \ldots, d Z_{m}(q)\right\}$ é uma base de $T_{q}^{\prime}$, para todo $q \in U$.

Em outras palavras, para cada $p \in \Omega$ é possivel obter uma carta $(U, \varphi)$, centrada em $p$, $\varphi=\left(x_{1}, \ldots, x_{N}\right)$; funções $Z_{1}, \ldots, Z_{m} \in C^{\infty}(U)$ e seções $L_{1}, \ldots, L_{n}$ de $\mathcal{V}$ sobre $U$ tais que

i) $\left\{d Z_{1}(q), \ldots, d Z_{m}(q)\right\}$ é linearmente independente para todo $q \in U$.

ii) $\left\{L_{1 q}, \ldots, L_{n q}\right\}$ é linearmente independente para todo $q \in U$.

iii) Se $L_{j}=\sum_{k=1}^{N} a_{j k} \frac{\partial}{\partial x_{k}}, j=1, \ldots, n$, ent $\tilde{a} o 0=d Z_{l}\left(L_{j}\right)=L_{j}\left(Z_{l}\right)=\sum_{k=1}^{N} a_{j k} \frac{\partial Z_{l}}{\partial x_{k}}$, para todo $j=1, \ldots, n$ e $l=1, \ldots, m$.

Observação 3.66. Dizemos que um operador é localmente integrável quando a estrutura gerada por ele for localmente integrável, analogamente falamos em sistema de operadores localmente integráveis. 
Exemplo 3.67. O operador de Mizohata $M=\frac{\partial}{\partial t}-i t \frac{\partial}{\partial x}$ é localmente integrável.

De fato, notemos que $M$ é não singular em $\mathbb{R}^{2}$, ou seja $M_{(x, t)} \neq 0$, para todo $(x, t) \in \mathbb{R}^{2}$. $\operatorname{Logo} \mathcal{V}=<M>$ é involutiva.

Por outro lado tomando $Z(x, t)=x+i \frac{t^{2}}{2}$, temos que $Z \in C^{\infty}\left(\mathbb{R}^{2}\right)$ e $d Z=d x+i t d t \neq 0$, para todo $(x, t) \in \mathbb{R}^{2}$, além disso,

$$
d Z(M)=M Z=\frac{\partial}{\partial t}\left(x+i \frac{t^{2}}{2}\right)-i t \frac{\partial}{\partial x}\left(x+i \frac{t^{2}}{2}\right)=i t-i t=0 .
$$

Portanto $\mathcal{V}$ é localmente integrável e $T^{\prime}=<d Z>$.

Proposição 3.68. Seja $L=\sum_{j=1}^{N} a_{j}(x) \frac{\partial}{\partial x_{j}}$ e $f \in C^{\infty}(U)$ tal que $L f=0$. Se $h \in \mathcal{O}(V)$, onde $V$ é aberto de $\mathbb{C}$ com $\operatorname{Im}(f) \subset V$, então $L(h \circ f)=0$. Mais geralmente se $L f_{j}=0$, $j=1, \ldots, m$ e $h \in \mathcal{O}(V), V \subseteq \mathbb{C}^{m}, \operatorname{com} \operatorname{Im}(f) \subset V$, onde $f=\left(f_{1}, \ldots, f_{m}\right)$, então $L(h \circ f)=0$.

Demonstração. Considere o seguinte diagrama

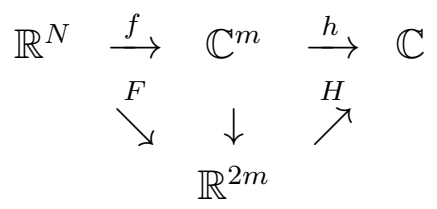

onde a seta vertical indica o isomorfismo $G$ entre $\mathbb{C}^{m}$ e $\mathbb{R}^{2 m}$. Logo, $F=G \circ f, H=h \circ G^{-1}$ e, além disso, $H \circ F=h \circ f$.

Aqui $\zeta=\left(\zeta_{1}, \ldots, \zeta_{m}\right) \operatorname{com} \zeta_{j}=u_{j}+i v_{j}, j=1, \ldots, m$ e $f_{j}(x)=u_{j}(x)+i v_{j}(x)$. Pela regra da cadeia temos

$$
\frac{\partial}{\partial x_{j}}(h \circ f)=\frac{\partial}{\partial x_{j}}(H \circ F)=\sum_{k=1}^{m}\left(\frac{\partial H}{\partial u_{k}} \cdot \frac{\partial u_{k}}{\partial x_{j}}+\frac{\partial H}{\partial v_{k}} \cdot \frac{\partial v_{k}}{\partial x_{j}}\right) .
$$

Logo,

$$
\begin{aligned}
L(h \circ f) & =\sum_{j=1}^{N} a_{j}(x) \frac{\partial}{\partial x_{j}}(h \circ f) \\
& =\sum_{j=1}^{N} a_{j}(x)\left(\sum_{k=1}^{m} \frac{\partial H}{\partial u_{k}} \cdot \frac{\partial u_{k}}{\partial x_{j}}+\frac{\partial H}{\partial v_{k}} \cdot \frac{\partial v_{k}}{\partial x_{j}}\right) \\
& =\sum_{k=1}^{m}\left[\left(\sum_{j=1}^{N} a_{j}(x) \frac{\partial u_{k}}{\partial x_{j}}\right) \frac{\partial H}{\partial u_{k}}+\left(\sum_{j=1}^{N} a_{j}(x) \frac{\partial v_{k}}{\partial x_{j}}\right) \frac{\partial H}{\partial v_{k}}\right] \\
& =\sum_{k=1}^{m}\left(L u_{k} \frac{\partial H}{\partial u_{k}}+L v_{k} \frac{\partial H}{\partial v_{k}}\right),
\end{aligned}
$$


de onde obtemos que

$$
L(h \circ f)=\sum_{k=1}^{m}\left(L u_{k} \frac{\partial H}{\partial u_{k}}+L v_{k} \frac{\partial H}{\partial v_{k}}\right) .
$$

Por outro lado, como $h$ é holomorfa então $\frac{\partial h}{\partial \bar{\zeta}_{k}}=0, k=1, \ldots, m$, mas

$$
0=\frac{\partial h}{\partial \bar{\zeta}_{k}}=\frac{1}{2}\left(\frac{\partial H}{\partial u_{k}}+i \frac{\partial H}{\partial v_{k}}\right),
$$

$\operatorname{logo}-i \frac{\partial H}{\partial v_{k}}=\frac{\partial H}{\partial u_{k}}$, o qual implica que $\frac{\partial H}{\partial v_{k}}=i \frac{\partial H}{\partial u_{k}}$. Portanto, de (3.1) temos

$$
\begin{aligned}
L(h \circ f) & =\sum_{k=1}^{m}\left(L u_{k} \frac{\partial H}{\partial u_{k}}+i L v_{k} \frac{\partial H}{\partial u_{k}}\right)=\sum_{k=1}^{m}\left(L u_{k}+i L v_{k}\right) \frac{\partial H}{\partial u_{k}} \\
& =\sum_{k=1}^{m}\left(L\left(u_{k}+i v_{k}\right)\right) \frac{\partial H}{\partial u_{k}}=\sum_{k=1}^{m}\left(L f_{k}\right) \frac{\partial H}{\partial u_{k}}=0,
\end{aligned}
$$

de onde obtemos que $L(h \circ f)=0$.

Observação 3.69. Um fato interessante que devemos observar é que na definição de estrutura localmente integrável não precisamos mencionar que $\mathcal{V}$ é involutiva, pois se $\mathcal{V}$ é um subfibrado que satisfaz a definição de estrutura localmente integrável, então ela é involutiva.

Proposição 3.70. Seja $\mathcal{V} \subseteq \mathbb{C} T \Omega$ um subfibrado e suponha que em cada $p \in \Omega$ existe uma carta $(U, \varphi)$, centrada em $p$ com $\varphi=\left(x_{1}, \ldots, x_{N}\right)$ e que existem seções de $\mathcal{V}, L_{1}, \ldots, L_{n}$ sobre $U$ e $Z_{1}, \ldots, Z_{m}$ funções $C^{\infty}(U)$ tais que $L_{j} Z_{l}=0, j=1, \ldots, n$ e $l=1, \ldots, m$. Onde $\mathcal{V}$ é gerado sobre $U$ pelas seções $L_{1}, \ldots, L_{n}$ e $T^{\prime}=\mathcal{V}^{\perp}$ é gerado sobre $U$ pelas 1-formas $d Z_{1}, \ldots, d Z_{m}$, então $\mathcal{V}$ é involutiva.

Demonstração. Notemos que para todo $j=1, \ldots, n$ e $l=1, \ldots, m$ temos

$$
d Z_{l}\left(\left[L_{j}, L_{k}\right]\right)=\left[L_{j}, L_{k}\right]\left(Z_{l}\right)=L_{j}\left(L_{k}\left(Z_{l}\right)\right)-L_{k}\left(L_{j}\left(Z_{l}\right)\right)=0
$$

pois $L_{j}, L_{k} \in \mathcal{V}$ e $d Z_{l} \in T^{\prime}=\mathcal{V}^{\perp}$.

Portanto $\left[L_{j}, L_{k}\right] \perp d Z_{l}$, para todo $j, k=1, \ldots, n$ e $l=1, \ldots, m$, o qual implica que $\left[L_{j}, L_{k}\right] \in\left(T^{\prime}\right)^{\perp}=\left(\mathcal{V}^{\perp}\right)^{\perp}=\mathcal{V}$ para todo $j, k=1, \ldots, n$. Logo $\mathcal{V}$ é involutiva.

Lema 3.71. Seja $V \subseteq \mathbb{C}^{N}$ um subespaço vetorial complexo e $V_{0}=V \cap \mathbb{R}^{N}$, então $V_{0}+i V_{0}=$ $V \cap \bar{V}$. 
Demonstração. Mostremos primeiro que $V_{0}+i V_{0} \subset V \cap \bar{V}$. De fato, sejam $x, y \in V_{0}=$ $V \cap \mathbb{R}^{N}$, em particular $x, y \in V$ e como $V$ é espaço vetorial complexo segue que $x+i y$, $x-i y \in V$. Por outro lado $x+i y=\overline{x-i y} \in \bar{V}$, portanto $x+i y \in V \cap \bar{V}, \forall x, y \in V_{0}$.

Mostremos agora que $V \cap \bar{V} \subset V_{0}+i V_{0}$. De fato, seja $z \in V \cap \bar{V}$, então $\bar{z} \in \overline{V \cap \bar{V}}=$ $V \cap \bar{V}, \operatorname{logo} \frac{1}{2}(z+\bar{z}), \frac{1}{2}(i \bar{z}-i z) \in V$, daí $\operatorname{Re}(z)=\frac{1}{2}(z+\bar{z}) \in V \cap \mathbb{R}^{N}=V_{0}$ e $\operatorname{Im}(z)=$ $\frac{1}{2}(i \bar{z}-i z) \in V \cap \mathbb{R}^{N}=V_{0}$, mas $z=\frac{1}{2}(z+\bar{z})+i \frac{1}{2}(i \bar{z}-i z) \in V_{0}+i V_{0}$.

Corolário 3.72. Se $T^{0}$ é o conjunto característico de uma estrutura involutiva $\mathcal{V}$ e $T^{\prime}$ é o subfibrado ortogonal, então para cada $p \in \Omega$ temos que

$$
T_{p}^{0}+i T_{p}^{0}=T_{p}^{\prime} \cap{\overline{T^{\prime}}}_{p}
$$

Demonstração. Como $T_{p}^{\prime}$ e um subespaço vetorial complexo e $T_{p}^{0}=T_{p}^{\prime} \cap T_{p}^{*} \Omega$, o resultado segue do Lema 3.71

Corolário 3.73. Uma estrutura involutiva $\mathcal{V}$ é elítica se e somente se $T_{p}^{\prime} \cap{\overline{T^{\prime}}}_{p}=0$.

Demonstração. $\mathcal{V}$ é elítica se e somente se $T_{p}^{0}=T_{p}^{\prime} \cap T_{p}^{*} \Omega=0$, para todo $p \in \Omega$ e pelo Corolário 3.72 isso é verdadeiro se e somente se $T_{p}^{\prime} \cap{\overline{T^{\prime}}}_{p}=0$.

\subsection{O teorema de Frobenius e geradores locais}

Nesta parte vamos obter um resultado que nos permite escolher coordenadas locais apropriadas e geradores locais do subfibrado vetorial ortogonal $T^{\prime}$ quando a estrutura $\mathcal{V}$ é localmente integrável (Teorema 3.78). Este resultado é de extrema utilidade.

Vamos iniciar mostrando o Teorema de Frobenius, para isso precisamos do resultado seguinte.

Teorema 3.74. Seja $L=\sum_{j=1}^{N} a_{j}(x) \frac{\partial}{\partial x_{j}}$ um campo vetorial real não singular na origem $0 \in \mathbb{R}^{N}$, isto é $a_{j} \in C^{\infty}\left(\mathbb{R}^{N}, \mathbb{R}\right) j=1, \ldots, N$ e $L_{0} \neq 0$. Então existem coordenadas locais $y_{1}, \ldots, y_{N}$ definida numa vizinhança da origem de $\mathbb{R}^{N}$, segundo as quais $L$ se escreve como $\tilde{L}=\frac{\partial}{\partial y_{1}}$

Demonstração. Podemos supor que $a_{1}(0) \neq 0$ (caso contrário uma permutação de variáveis resolve o problema). Considere o seguinte P.V.I

$$
\left\{\begin{array}{l}
\frac{d x_{j}}{d t}=a_{j}\left(x_{1}, \ldots, x_{N}\right) \quad, \quad j=1, \ldots, N \\
x_{1}\left(0, y_{2}, \ldots, y_{N}\right)=0 \\
x_{j}\left(0, y_{2}, \ldots, y_{N}\right)=y_{j} \quad, \quad j=2, \ldots, N
\end{array}\right.
$$


Pelo Teorema de existência, unicidade e dependência $C^{\infty}$ de soluções de E.D.O obtemos a única solução $\left(t, y_{2}, \ldots, y_{N}\right)$ do P.V.I acima a qual é $C^{\infty}$ em uma vizinhança da origem. Como a matriz jacobiana na origem é dada por

$$
J=\left[\begin{array}{ccccc}
a_{1}(0) & 0 & 0 & \cdots & 0 \\
a_{2}(0) & 1 & 0 & \cdots & 0 \\
a_{3}(0) & 0 & 1 & \cdots & 0 \\
\vdots & \vdots & \vdots & \ddots & \vdots \\
a_{N}(0) & 0 & 0 & \cdots & 1
\end{array}\right]
$$

e $\operatorname{det}(J)=a_{1}(0) \neq 0$, então pelo Teorema da função inversa obtemos $t=t(x)$ e $y_{j}=y_{j}(x)$, $j=2, \ldots, N$, ou seja

$$
\left\{\begin{aligned}
t & =t\left(x_{1}(t, y), \ldots, x_{N}(t, y)\right) \\
y_{j} & =y_{j}\left(x_{1}(t, y), \ldots, x_{N}(t, y)\right) \quad, \quad j=2, \ldots, N
\end{aligned}\right.
$$

Derivando ambas as expressões acima em relação a $t$ obtemos o seguinte

$$
\left\{\begin{aligned}
1 & =\sum_{k=1}^{N} \frac{\partial t}{\partial x_{k}} \frac{\partial x_{k}}{\partial t}=\sum_{j=1}^{N} a_{k} \frac{\partial t}{\partial x_{k}}=L(t) \\
0 & =\sum_{k=1}^{N} \frac{\partial y_{j}}{\partial x_{k}} \frac{\partial x_{k}}{\partial t}=\sum_{k=1}^{N} a_{k} \frac{\partial y_{j}}{\partial x_{k}}=L\left(y_{j}\right), j=2, \ldots, N .
\end{aligned}\right.
$$

Agora se colocarmos $y_{1}=t$, nas novas coordenadas $\left(y_{1}, y_{2}, \ldots, y_{N}\right)$ teremos

$$
L=\sum_{j=1}^{N} L\left(y_{j}\right) \frac{\partial}{\partial y_{j}}=\frac{\partial}{\partial y_{1}}
$$

\section{Teorema 3.75. (Frobenius)}

Sejam $L_{1}, \ldots, L_{n}$ campos reais linearmente independentes em cada ponto de uma vizinhança $V$ da origem de $\mathbb{R}^{N}$. Suponha que o subfibrado $\mathcal{V}$ de $\mathbb{C} T V$ definido por estes campos seja uma estrutura involutiva. Então existem coordenadas locais $y_{1}, y_{2}, \ldots, y_{N}$ em torno da origem de $\mathbb{R}^{N}$ tais que numa vizinhança da origem, $\mathcal{V}$ é gerado pelos campos $\frac{\partial}{\partial y_{1}}, \ldots, \frac{\partial}{\partial y_{N}}$.

Demonstração. A demonstração segue por indução sobre a dimensão $N$.

No caso $N=1$ temos $L=a\left(x_{1}\right) \frac{\partial}{\partial x_{1}}$ com $a\left(x_{1}\right) \neq 0$ numa vizinhança da origem, portanto $\mathcal{V}=<\frac{\partial}{\partial x_{1}}>$ nesta vizinhança. 
Suponhamos agora que o teorema seja verdadeiro para qualquer natural menor do que $N$. Fazendo uma mudança de variáveis conveniente (sugerido pelo Teorema 3.74), podemos assumir que os campos acima se escrevem

$$
\begin{aligned}
L_{1} & =\frac{\partial}{\partial x_{1}} \\
L_{j} & =\sum_{k=1}^{N} a_{j k} \frac{\partial}{\partial x_{k}}, \quad j=2, \ldots, n .
\end{aligned}
$$

Logo sejam $L_{1}^{\sharp}=L_{1}$ e $L_{j}^{\sharp}=L_{j}-a_{j 1} L_{1}, j=2, \ldots, n$ então $L_{1}^{\sharp}, \ldots, L_{n}^{\sharp}$ geram $\mathcal{V}$ numa vizinhança da origem de $\mathbb{R}^{N}$ (basta ver que $L_{1}^{\sharp}, \ldots, L_{n}^{\sharp}$ são l.i e que $L_{1}^{\sharp}=\frac{\partial}{\partial x_{1}}$ afeta apenas as derivadas em relação a $x_{1}$ ).

Teremos assim que

$$
\left\{\begin{aligned}
L_{1}^{\sharp} & =\frac{\partial}{\partial x_{1}} \\
L_{j}^{\sharp} & =\sum_{k=2}^{N} a_{j k} \frac{\partial}{\partial x_{k}}, \quad j=2, \ldots, n
\end{aligned}\right.
$$

além disso, pela involutividade temos que

$$
\left[L_{j}^{\sharp}, L_{k}^{\sharp}\right]=\sum_{\nu=2}^{N} c_{j k}^{\nu} L_{\nu}^{\sharp}, \quad j, k=2, \ldots, n
$$

Agora considere os campos

$$
M_{j}=\sum_{k=2}^{N} a_{j k}\left(0, x_{2}, \ldots, x_{N}\right) \frac{\partial}{\partial x_{k}}, \quad j=2, \ldots, n
$$

definidos numa vizinhança $W$ da origem em $\mathbb{R}^{N-1}$. Aqui a variável $x_{1}=0$ (congelada em zero).

Devido a (3.2) acima, o subfibrado $\mathcal{V}^{0}$ de $\mathbb{C} T W$ gerado por $M_{2}, \ldots, M_{n}$ também é uma estrutura involutiva de dimensão $n-1 \mathrm{em} \mathbb{R}^{N-1}$, segue por indução que existem coordenadas $y_{2}, \ldots, y_{N}$ segundo as quais $\mathcal{V}^{0}=<\frac{\partial}{\partial y_{2}}, \ldots, \frac{\partial}{\partial y_{n}}>$. Portanto, como $M_{j}$ é seção de $\mathcal{V}^{0}$ sobre $W$ então temos que

$$
M_{j}=\sum_{k=2}^{n} b_{j k}\left(y_{2}, \ldots, y_{N}\right) \frac{\partial}{\partial y_{k}} ; \quad j=2, \ldots, n .
$$


Assim, retornando as coordenadas originais $\left(x_{1}, \ldots, x_{N}\right)$, podemos assumir que

$$
L_{j}^{\sharp}=\sum_{k=2}^{N} a_{j k}\left(x_{1}, \ldots, x_{N}\right) \frac{\partial}{\partial x_{k}} ; \quad j=2, \ldots, n
$$

e em $x_{1}=0$ temos que

$$
L_{j}^{\sharp}=\sum_{k=2}^{n} a_{j k}\left(0, x_{2}, \ldots, x_{N}\right) \frac{\partial}{\partial x_{k}} ; \quad j=2, \ldots, n
$$

ou seja, $a_{j k}\left(0, x_{2}, \ldots, x_{N}\right)=0$ para $j=2, \ldots, n$ e $k=n+1, \ldots, N$.

Vamos mostrar agora que $a_{j k}\left(x_{1}, \ldots, x_{N}\right)=0$ para $j=2, \ldots, n$ e $k=n+1, \ldots, N$.

Notemos que para $j=2, \ldots, n$ temos

$$
\begin{aligned}
{\left[L_{1}^{\sharp}, L_{j}^{\sharp}\right] } & =L_{1}^{\sharp} L_{j}^{\sharp}-L_{j}^{\sharp} L_{1}^{\sharp} \\
& =\frac{\partial}{\partial x_{1}}\left(\sum_{k=2}^{N} a_{j k}(x) \frac{\partial}{\partial x_{k}}\right)-\sum_{k=2}^{N} a_{j k}(x) \frac{\partial}{\partial x_{k}} \frac{\partial}{\partial x_{1}} \\
& =\sum_{k=2}^{N} \frac{\partial}{\partial x_{1}} a_{j k}(x) \frac{\partial}{\partial x_{k}}+\sum_{k=2}^{N} a_{j k}(x) \frac{\partial}{\partial x_{1}} \frac{\partial}{\partial x_{k}}-\sum_{k=2}^{N} a_{j k}(x) \frac{\partial}{\partial x_{k}} \frac{\partial}{\partial x_{1}} \\
& =\sum_{k=2}^{N} \frac{\partial}{\partial x_{1}} a_{j k}(x) \frac{\partial}{\partial x_{k}} .
\end{aligned}
$$

Por outro lado pela involutividade de $\mathcal{V}$ temos para $j=2, \ldots, n$ que

$$
\begin{aligned}
{\left[L_{1}^{\sharp}, L_{j}^{\sharp}\right] } & =\sum_{\nu=1}^{n} c_{1 j}^{\nu} L_{\nu}^{\sharp} \\
& =c_{1 j}^{1} L_{1}^{\sharp}+\sum_{\nu=2}^{n} c_{1 j}^{\nu} L_{\nu}^{\sharp} \\
& =c_{1 j}^{1} \frac{\partial}{\partial x_{1}}+\sum_{\nu=2}^{n} c_{1 j}^{\nu}\left(\sum_{k=2}^{N} a_{\nu k}(x) \frac{\partial}{\partial x_{k}}\right) \\
& =c_{1 j}^{1} \frac{\partial}{\partial x_{1}}+\sum_{k=2}^{N}\left(\sum_{\nu=2}^{n} c_{1 j}^{\nu} a_{\nu k}(x)\right) \frac{\partial}{\partial x_{k}} .
\end{aligned}
$$

Resumindo, temos

$$
\left[L_{1}^{\sharp}, L_{j}^{\sharp}\right]=\sum_{k=2}^{N} \frac{\partial}{\partial x_{1}} a_{j k}(x) \frac{\partial}{\partial x_{k}}
$$




$$
\left[L_{1}^{\sharp}, L_{j}^{\sharp}\right]=c_{1 j}^{1} \frac{\partial}{\partial x_{1}}+\sum_{k=2}^{N}\left(\sum_{\nu=2}^{n} c_{1 j}^{\nu} a_{\nu k}(x)\right) \frac{\partial}{\partial x_{k}} .
$$

Portanto, das equações (3.3) e (3.4) segue que

$$
\frac{\partial a_{j k}}{\partial x_{1}}=\sum_{\nu=2}^{n} c_{1 j}^{\nu} a_{\nu k} \quad ; \quad k=2, \ldots, N \text { e } j=2, \ldots, n .
$$

Agora para $k=n+1, \ldots, N$ o P.V.I seguinte

$$
\left\{\begin{array}{l}
\frac{d \tilde{a}_{j k}}{d x_{1}}=\sum_{\nu=2}^{N} c_{1 j}^{\nu} \tilde{a}_{\nu k} \\
\left.\tilde{a}_{j k}(x)\right|_{x_{1}=0}=0 ; \quad j=2, \ldots, n
\end{array}\right.
$$

tem como solução os vetores $\left(a_{2 k}, \ldots, a_{n k}\right)$ e $(0,0, \ldots, 0)$ e pela unicidade de soluções para o P.V.I vem que $a_{j k}(x)=0$ numa vizinhança da origem para $j=2, \ldots, n$ e $k=n+1, \ldots, N$. Desta forma temos que

$$
L_{1}^{\sharp}=\frac{\partial}{\partial x_{1}} \quad L_{j}^{\sharp}=\sum_{k=2}^{n} a_{j k}(x) \frac{\partial}{\partial x_{k}} ; j=2, \ldots, n .
$$

Isto é, $\mathcal{V}$ é gerado pelos campos $\frac{\partial}{\partial x_{1}}, \ldots, \frac{\partial}{\partial x_{n}}$, o que prova o teorema.

Lema 3.76. Seja $V \subseteq \mathbb{C}^{N}$ um subespaço vetorial complexo com $\operatorname{dim}(V)=m$ e seja $V_{0}=V \cap \mathbb{R}^{N}$ onde $d=\operatorname{dim}_{\mathbb{R}}\left(V_{0}\right)$ e $\nu=m-d \geq 0$. Seja $V_{1} \subseteq \mathbb{C}^{N}$ o subespaço vetorial tal que $\left(V_{0}+i V_{0}\right) \oplus V_{1}=V$, considere $\left\{\zeta_{1}, \ldots, \zeta_{\nu}\right\}$ base de $V_{1}$ e $\left\{\xi_{\nu+1}, \ldots, \xi_{m}\right\}$ base real de $V_{0}$. Se escrevemos $\zeta_{j}=\xi_{j}+i \eta_{j}, j=1, \ldots, \nu$ então

a) $\left\{\zeta_{1}, \ldots, \zeta_{\nu}, \xi_{\nu+1}, \ldots, \xi_{m}\right\}$ é base de $V$.

b) $\left\{\xi_{1}, \ldots, \xi_{\nu}, \xi_{\nu+1}, \ldots, \xi_{m}, \eta_{1}, \ldots, \eta_{\nu}\right\}$ é l.i sobre $\mathbb{R}$.

c) $\nu+m \leq N$.

Demonstração. Mostremos a). Como $\left\{\zeta_{1}, \ldots, \zeta_{\nu}\right\}$ é base (complexa) de $V_{1}$ e $\left\{\xi_{\nu+1}, \ldots, \xi_{m}\right\}$ é base (real) de $V_{0}$, então $\left\{\xi_{\nu+1}, \ldots, \xi_{m}\right\}$ é base complexa de $V_{0}+i V_{0}$ (complexa no sentido de podermos usar coeficientes complexos nas combinações lineares), mas $\left(V_{0}+i V_{0}\right) \cap V_{1}=$ $\{0\}$ e $V=\left(V_{0}+i V_{0}\right) \oplus V_{1}$, então segue que $\left\{\zeta_{1}, \ldots, \zeta_{\nu}, \xi_{\nu+1}, \ldots, \xi_{m}\right\}$ é base de $V$.

Para mostrar b) notemos primeiro que $V \cap \bar{V}_{1}=0$. De fato, se $z=x+i y \in V \cap \bar{V}_{1}$, então $\bar{z}=x-i y \in V_{1} \subset V$, ou seja $z, \bar{z} \in V \operatorname{logo} x=\frac{1}{2}(z+\bar{z}) \in V \cap \mathbb{R}^{N}=V_{0} \mathrm{e}$ $y=\frac{1}{2 i}(z-\bar{z}) \in V \cap \mathbb{R}^{N}=V_{0}, \log 0 x, y \in V_{0}$ e $\bar{z}=x-i y=x+i(-y) \in V_{0}+i V_{0}$. Daí 
$\bar{z} \in V_{1} \cap\left(V_{0}+i V_{0}\right)=\{0\}$, então $\bar{z}=0$, o qual implica que $z=0$. Portanto $V \cap \bar{V}_{1}=0$. Agora, como $\left\{\zeta_{1}, \ldots, \zeta_{\nu}\right\}$ é base de $V_{1}$ então $\left\{\bar{\zeta}_{1}, \ldots, \bar{\zeta}_{\nu}\right\}$ é base de $\bar{V}_{1}$ e pelo item $\left.a\right)$ temos que $\left\{\zeta_{1}, \ldots, \zeta_{\nu}, \xi_{\nu+1}, \ldots, \xi_{m}\right\}$ é base de $V$, então $\beta=\left\{\zeta_{1}, \ldots, \zeta_{\nu}, \xi_{\nu+1}, \ldots, \xi_{m}, \bar{\zeta}_{1}, \ldots, \bar{\zeta}_{\nu}\right\}$ é base de $V \oplus \bar{V}_{1}$. Mas $\zeta_{j}=\xi_{j}+i \eta_{j}, j=1, \ldots, \nu$. Portanto, recombinando os elementos de $\beta$ obtemos uma nova base $\beta^{\prime}=\left\{\xi_{1}, \ldots, \xi_{\nu}, \xi_{\nu+1}, \ldots, \xi_{m}, \eta_{1}, \ldots, \eta_{\nu}\right\}$ para $V \oplus \bar{V}_{1}$, em particular este conjunto é l.i sobre $\mathbb{C}$ e, portanto, l.i sobre $\mathbb{R}$.

Finalmente para mostrar $c$ ) basta observar que como o conjunto $\beta^{\prime}$ é uma base complexa de $V \oplus \bar{V}_{1} \subseteq \mathbb{C}^{N}$, então $\nu+m \leq N$.

Corolário 3.77. Nas mesmas hipóteses do Lema 3.76, a seguinte igualdade se satisfaz

$$
V \oplus \bar{V}_{1}=V \oplus \bar{V}
$$

Demonstração.

$$
\begin{aligned}
V \oplus \bar{V} & =\left[\left(V_{0}+i V_{0}\right) \oplus V_{1}\right] \oplus \overline{\left[\left(V_{0}+i V_{0}\right) \oplus V_{1}\right]} \\
& =\left[\left(V_{0}+i V_{0}\right) \oplus V_{1}\right] \oplus\left[\left(V_{0}+i V_{0}\right) \oplus \bar{V}_{1}\right] \\
& =V \oplus \bar{V}_{1} .
\end{aligned}
$$

Teorema 3.78. (geradores locais)

Seja $\mathcal{V} \subseteq \mathbb{C} T \Omega$ uma estrutura localmente integrável definida sobre a variedade $\Omega, p \in \Omega$ e $d=\operatorname{dim}_{\mathbb{R}} T_{p}^{0}$. Então existe um sistema de coordenadas centrado em $p$, zerando em $p$

$$
\left\{x_{1}, \ldots, x_{\nu}, y_{1}, \ldots, y_{\nu}, s_{1}, \ldots, s_{d}, t_{1}, \ldots, t_{n^{\prime}}\right\}
$$

e funções $\varphi_{1}, \ldots, \varphi_{d}$ de classe $C^{\infty}$ definidas numa vizinhança da origem e a valores reais satisfazendo

$$
\varphi_{k}(0)=0=d \varphi_{k}(0)=0, \quad k=1, \ldots, d,
$$

tais que os diferenciais das funções

$$
\begin{aligned}
Z_{j}(x, y) & =z_{j}=x_{j}+i y_{j}, \quad j=1, \ldots, \nu \\
W_{k}(x, y, s, t) & =s_{k}+i \varphi_{k}(x, y, s, t), \quad k=1, \ldots, d,
\end{aligned}
$$

geram $T^{\prime}$ em uma vizinhança da origem. Em particular, temos que $\nu+d=m, \nu+n^{\prime}=n$ e também

$$
T_{p}^{0}=<\left.d s_{1}\right|_{0}, \ldots,\left.d s_{d}\right|_{0}>
$$


Demonstração. Seja $\mathcal{V}$ uma estrutura localmente integrável sobre $\Omega, p \in \Omega$ e $G_{1}, \ldots, G_{m} \in$ $C^{\infty}(W)$, com $W$ vizinhança de $p$ tais que $\left\{d G_{1}(q), \ldots, d G_{m}(q)\right\}$ é base de $T_{q}^{\prime}$, para todo $q \in W$. Vamos aplicar o Lema $3.76 \operatorname{com} V=T_{p}^{\prime} \subseteq \mathbb{C} T_{p}^{*} \Omega \cong \mathbb{C}^{N}$ e $V_{0}=T_{p}^{\prime} \cap T_{p}^{*} \Omega=T_{p}^{0}$. Seja $\left\{\zeta_{1}, \ldots, \zeta_{\nu}, \xi_{\nu+1}, \ldots, \xi_{m}\right\}$ a base de $T_{p}^{\prime}$ (como no item a) do Lema 3.76). Então existe uma matriz invertível $\left(c_{j k}\right)_{m \times m}$ (matriz mudança de base), tal que

$$
\left\{\begin{array}{l}
\sum_{k=1}^{m} c_{j k} d G_{k}(p)=\zeta_{j} \quad, \quad j=1, \ldots, \nu \\
\sum_{k=1}^{m} c_{j k} d G_{k}(p)=\xi_{j} \quad, \quad j=\nu+1, \ldots, m
\end{array}\right.
$$

ou seja,

$$
\left(c_{j k}\right) \cdot\left[\begin{array}{c}
d G_{1}(p) \\
\vdots \\
d G_{\nu}(p) \\
d G_{\nu+1}(p) \\
\vdots \\
d G_{m}(p)
\end{array}\right]=\left[\begin{array}{c}
\zeta_{1} \\
\vdots \\
\zeta_{\nu} \\
\xi_{\nu+1} \\
\vdots \\
\xi_{m}
\end{array}\right]
$$

Definimos

$$
\begin{aligned}
& Z_{j}(q)=\sum_{k=1}^{m} c_{j, k}\left(G_{k}(q)-G_{k}(p)\right) \quad, \quad q \in W, j=1, \ldots, \nu \\
& W_{l}(q)=\sum_{k=1}^{m} c_{\nu+l, k}\left(G_{k}(q)-G_{k}(p)\right) \quad, \quad q \in W, l=1, \ldots, d .
\end{aligned}
$$

Temos assim por (3.5) que $d Z_{1}, \ldots, d Z_{\nu}, d W_{1}, \ldots, d W_{d}$ geram $T^{\prime}$ numa vizinhança de $p$. Pela conclusão $b$ ) do Lema 3.76, com $Z_{j}=x_{j}+i y_{j} ; d Z_{j}=\zeta_{j}=d x_{j}+i d y_{j}, j=1, \ldots, \nu$; $d W_{l}(p)=\xi_{l}$ e $s_{l}=R e\left(W_{l}\right)$, temos que

$$
\left\{d x_{1}(p), \ldots, d x_{\nu}(p), d y_{1}(p), \ldots, d y_{\nu}(p), d s_{1}(p), \ldots, d s_{d}(p)\right\}
$$

é um conjunto linearmente independente sobre $\mathbb{R}$.

Agora escolhamos $t_{1}, \ldots, t_{n^{\prime}}$ funções $C^{\infty}$ a valores reais definidas numa vizinhança de $p$ com $t_{k}(p)=0, k=1, \ldots, n^{\prime}$ onde $\nu+n^{\prime}=n$ de modo que $d x_{1}, \ldots, d x_{\nu}, d y_{1}, \ldots, d y_{\nu}, d s_{1}, \ldots, d s_{d}$, $d t_{1}, \ldots, d t_{n^{\prime}}$ sejam linearmente independentes em $p$. Logo $(x, y, s, t)$ é um sistema de co- 
ordenadas centrada em $p$ e anulando-se em $p$. Temos, portanto,

$$
\left\{\begin{array}{l}
Z_{j}=x_{j}+i y_{j} \quad, \quad j=1, \ldots, \nu \\
W_{l}=s_{l}+i \varphi_{l}(x, y, s, t)
\end{array}\right.
$$

e $d Z_{1}, \ldots, d Z_{\nu}, d W_{1}, \ldots, d W_{d}$ geram $T^{\prime}$ numa vizinhança de $p$, além disso,

$$
\begin{cases}x_{j}(p)=y_{j}(p) & =0, \quad j=1, \ldots, \nu \\ s_{l}(p) & =0, \quad l=1, \ldots, d \\ t_{k}(p) & =0, \quad k=1, \ldots, n^{\prime}\end{cases}
$$

daí

$$
\begin{aligned}
\varphi_{l}(0) & =\varphi_{l}(x(p), y(p), s(p), t(p))=\varphi_{l}(0,0,0,0)=0,\left(\text { pois } \omega_{l}(0)=0\right) \quad \mathrm{e} \\
d W_{l}(p) & =d s_{l}(p)+i d \varphi_{l}(x(p), y(p), s(p), t(p))=\xi_{\nu+l} \text { real } .
\end{aligned}
$$

$\operatorname{Logo} d \varphi_{l}(0,0,0,0)=0, l=1, \ldots, d$.

O seguinte corolário é uma consequência imediata do teorema anterior.

Corolário 3.79. Seja $\mathcal{V} \subseteq \mathbb{C} T \Omega$ uma estrutura localmente integrável, $p \in \Omega$. Então existe um sistema de coordenadas zerando em $p\left\{x_{1}, \ldots, x_{m}, t_{1}, \ldots, t_{n}\right\}$ e funções a valores reais suaves $\varphi_{1}, \ldots, \varphi_{m}$ definidas numa vizinhança da origem satisfazendo

$$
\varphi_{k}(0,0)=0 \quad d_{x} \varphi_{k}(0,0)=0, k=1, \ldots, m
$$

tais que os diferenciais das funções $Z_{k}(x, t)=x_{k}+i \varphi_{k}(x, t)$ geram $T^{\prime}$ em uma vizinhança da origem.

Aplicação: Seja $\mathcal{V} \subseteq \mathbb{C} T \Omega$ uma estrutura localmente integrável segundo o corolário anterior existe um sistema de coordenadas zerando em $p,(x, t)=\left(x_{1}, \ldots, x_{m}, t_{1}, \ldots, t_{n}\right) \mathrm{e}$ existem funções reais $\varphi_{k}$ de classe $C^{\infty}$ numa vizinhança da origem satisfazendo $\varphi_{k}(0,0)=$ 0 e $d_{x} \varphi_{k}(0,0)=0, k=1, \ldots, m$, tais que os diferenciais das funções $Z_{k}(x, t)=x_{k}+$ $i \varphi_{k}(x, t), k=1, \ldots, m$ geram $T^{\prime}$ numa vizinhança de $p$.

Agora vamos definir alguns campos vetoriais numa vizinhança da origem do $\mathbb{R}^{N}$ pondo

$$
M_{k}=\sum_{l=1}^{m} M_{k l}(x, t) \frac{\partial}{\partial x_{l}} \quad, \quad k=1, \ldots, m,
$$

de modo que $M_{k} Z_{j}=\delta_{k j}, k=1, \ldots, m$. Ou seja, os coeficientes $M_{k l}$ são definidos pela 
igualdade de matrizes abaixo

$$
\left[\sum_{l=1}^{m} M_{k l} \frac{\partial Z_{j}}{\partial x_{l}}\right]_{1 \leq k, j \leq m}=I_{m \times m}
$$

$\mathrm{Na}$ matriz do lado esquerdo da igualdade acima, fixando um $(k, j)$ e somando em $l$ obtemos o $(k, j)$-ésimo elemento do produto de $\left[M_{k l}\right]_{m \times m}$ pela transposta de $\left[\frac{\partial Z_{j}}{\partial x_{l}}\right]_{m \times m}$, ou seja, para determinarmos a matriz $\left[M_{k j}\right]_{m \times m}$ precisamos resolver o sistema linear seguinte

$$
\left[M_{k l}\right]_{m \times m}\left[\frac{\partial Z_{j}}{\partial x_{l}}\right]_{m \times m}^{t}=\left[\sum_{l=1}^{m} M_{k l} \frac{\partial Z_{j}}{\partial x_{l}}\right]_{m \times m}=I_{m \times m} .
$$

Como os diferenciais das funções $Z_{k}$ geram $T^{\prime}$ numa vizinhança da origem então os vetores $d Z_{k}=\sum_{l=1}^{m} \frac{\partial Z_{k}}{\partial x_{l}} d x_{l}$ são linearmente independentes nesta vizinhança. Em outras palavras a matriz $\left[\frac{\partial Z_{j}}{\partial x_{l}}\right]_{m \times m}^{t}$ é invertível, logo

$$
\left[M_{k l}\right]_{m \times m}=\left[\left[\frac{\partial Z_{j}}{\partial x_{l}}\right]^{t}\right]^{-1}
$$

Agora se $Z=\left(Z_{1}, \ldots, Z_{m}\right)$, pelo corolário acima temos que

$$
Z_{x}(0,0)=\left[\frac{\partial Z_{j}}{\partial x_{l}}(0,0)\right]_{m \times m}=I_{x}+i\left[\frac{\partial \varphi_{j}}{\partial x_{l}}(0,0)\right]=I_{m \times m}
$$

onde a última igualdade é satisfeita porque $d_{x} \varphi_{k}(0,0)=0$, para $k=1, \ldots, m$.

Defina

$$
L_{j}=\frac{\partial}{\partial t_{j}}-i \sum_{l=1}^{m} \frac{\partial \varphi_{l}(x, t)}{\partial t_{j}} M_{l}
$$

Então temos que

$$
\begin{cases}L_{1}, L_{2}, \ldots, L_{n} & \text { são } 1 . \mathrm{i} \\ L_{j} Z_{k}=0 & j=1, \ldots, n \text { e } k=1, \ldots, m\end{cases}
$$

De fato, para mostrar que $L_{1}, \ldots, L_{n}$ são l.i, sejam $\alpha_{1}, \ldots, \alpha_{n} \in \mathbb{C}$ tais que

$$
L=\sum_{\nu=1}^{n} \alpha_{\nu} L_{\nu} \equiv 0
$$


e seja $\psi_{j}(x, t)=t_{j}, j=1, \ldots, n$ então

$$
0=L t_{j}=\sum_{\nu=1}^{n} \alpha_{\nu} L_{\nu} t_{j}=\alpha_{j}, \quad j=1, \ldots, n
$$

Mostremos agora que $L_{j} Z_{k}=0$ :

$$
\begin{aligned}
L_{j} Z_{k} & =\left(\frac{\partial}{\partial t_{j}}-i \sum_{l=1}^{m} \frac{\partial \varphi_{l}}{\partial t_{j}}(x, t) M_{l}\right)\left(Z_{k}\right)=\frac{\partial}{\partial t_{j}}\left(Z_{k}\right)-i \sum_{l=1}^{m} \frac{\partial \varphi_{l}}{\partial t_{j}} M_{l}\left(Z_{k}\right) \\
& =\frac{\partial}{\partial t_{j}}\left(x_{k}+i \varphi_{k}(x, t)\right)-i \sum_{l=1}^{m} \frac{\partial \varphi_{l}}{\partial t_{j}} \delta_{k l}=i \frac{\partial \varphi_{k}}{\partial t_{j}}-i \frac{\partial \varphi_{k}}{\partial t_{j}}=0
\end{aligned}
$$

Portanto,

$$
L_{j} Z_{k}=0, \operatorname{para} j=1, \ldots, n \text { e } k=1, \ldots, m .
$$

De tudo o feito na aplicação anterior temos as seguintes conclusões:

Proposição 3.80. Sejam os campos $M_{k}$ e $L_{j}$ definidos nas equações (3.6) e (3.7) respectivamente, então temos as seguintes conclusões:

a) Os campos $L_{1}, \ldots, L_{n}, M_{1}, \ldots, M_{m}$ geram $\mathbb{C} T \mathbb{R}^{N}$ numa vizinhança da origem.

b) $\mathcal{V}=<L_{1}, \ldots, L_{n}>$.

c) A base dual de $\left\{L_{1}, \ldots, L_{n}, M_{1}, \ldots, M_{m}\right\}$ é $\left\{d t_{1}, \ldots, d t_{n}, d Z_{1}, \ldots, d Z_{m}\right\}$ (base de $\left.\mathbb{C} T^{*} \Omega\right)$.

d) Os campos $L_{1}, \ldots, L_{n}, M_{1}, \ldots, M_{m}$ comutam entre si.

e) $S e f \in C^{1}$, então

$$
d f=\sum_{j=1}^{n} L_{j} f d t_{j}+\sum_{k=1}^{m} M_{k} f d Z_{k}
$$

Demonstração. Mostremos a). Como sabemos, a matriz $\left[M_{k l}\right]_{m \times m}$ que define os campos $M_{k}$ é invertível, então os campos $M_{1}, \ldots, M_{m}$ são l.i e por definição não dependem das variáveis $t_{1}, \ldots, t_{n}$ o que garante a independência linear com os campos $L_{1}, \ldots, L_{n}$.

Segue-se que o conjunto $\left\{L_{1}, \ldots, L_{n}, M_{1}, \ldots, M_{m}\right\}$ é linearmente independente em $\mathbb{C} T \mathbb{R}^{N}$, e $m+n=N$, ou seja, geram $\mathbb{C} T \mathbb{R}^{N}$ numa vizinhança da origem.

Para mostrar $b$ ). Pelo Corolário 3.79 os diferenciais $d Z_{1}, \ldots, d Z_{m}$ geram $T^{\prime}$ numa vizinhança da origem e

$$
d Z_{k}\left(L_{j}\right)=L_{j}\left(Z_{k}\right)=0 \quad, \quad k=1, \ldots, m \quad j=1, \ldots, n .
$$


Portanto, $\left\{d Z_{1}, \ldots, d Z_{m}\right\}$ é base dual de $\left\{L_{1}, \ldots, L_{n}\right\}$, ou seja,

$$
<L_{1}, \ldots, L_{n}>=\left(T^{\prime}\right)^{\perp}=\left(\mathcal{V}^{\perp}\right)^{\perp}=\mathcal{V}
$$

Para verificar $c$ ) basta observar que

$$
\begin{array}{rlll}
d Z_{j}\left(M_{l}\right) & =M_{l}\left(Z_{j}\right)=\delta_{l j} & j, l=1, \ldots, m \\
d Z_{j}\left(L_{k}\right)=L_{k}\left(Z_{j}\right)=0 \quad j=1, \ldots, m \quad k=1, \ldots, n \\
d t_{k}\left(L_{j}\right)=L_{j}\left(t_{k}\right)=\delta_{j k} & j, k=1, \ldots, n \\
d t_{k}\left(M_{j}\right)=M_{j}\left(t_{k}\right)=0 \quad j=1, \ldots, m \quad k=1, \ldots, n .
\end{array}
$$

Para mostrar $d$ ) vamos usar o item $c$ ) e o seguinte

$$
\begin{aligned}
& d t_{l}\left(\left[L_{j}, L_{k}\right]\right)=\left[L_{j}, L_{k}\right]\left(t_{l}\right)=L_{j}\left(L_{k} t_{l}\right)-L_{k}\left(L_{j} t_{l}\right)=L_{j}\left(\delta_{k l}\right)-L_{k}\left(\delta_{j l}\right)=0 \\
& d t_{l}\left(\left[L_{j}, M_{k}\right]\right)=\left[L_{j}, M_{k}\right]\left(t_{l}\right)=L_{j}\left(M_{k} t_{l}\right)-M_{k}\left(L_{j} t_{l}\right)=L_{j}(0)-M_{k}\left(\delta_{j l}\right)=0 \\
& d t_{l}\left(\left[M_{j}, M_{k}\right]\right)=\left[M_{j}, M_{k}\right]\left(t_{l}\right)=M_{j}\left(M_{k} t_{l}\right)-M_{k}\left(M_{j} t_{l}\right)=L_{j}(0)-L_{k}(0)=0 \\
& d Z_{l}\left(\left[L_{j}, L_{k}\right]\right)=\left[L_{j}, L_{k}\right]\left(Z_{l}\right)=L_{j}\left(L_{k} Z_{l}\right)-L_{k}\left(L_{j} Z_{l}\right)=L_{j}(0)-L_{k}(0)=0 \\
& d Z_{l}\left(\left[L_{j}, M_{k}\right]\right)=\left[L_{j}, M_{k}\right]\left(Z_{l}\right)=L_{j}\left(M_{k} Z_{l}\right)-M_{k}\left(L_{j} Z_{l}\right)=L_{j}\left(\delta_{k l}\right)-L_{k}(0)=0 \\
& d Z_{l}\left(\left[M_{j}, M_{k}\right]\right)=\left[M_{j}, M_{k}\right]\left(Z_{l}\right)=M_{j}\left(M_{k} Z_{l}\right)-M_{k}\left(M_{j} Z_{l}\right)=M_{j}\left(\delta_{k l}\right)-M_{k}\left(\delta_{j l}\right)=0 .
\end{aligned}
$$

Estas contas mostram que se $X, Y \in\left\{L_{1}, \ldots, L_{n}, M_{1}, \ldots, M_{m}\right\}$, então $[X, Y] \perp \mathbb{C} T^{*} U$ onde $U$ é uma vizinhança da origem. Logo $[X, Y] \in\left(\mathbb{C} T^{*} U\right)^{\perp}=\{0\}$, então $[X, Y]=0$ portanto $L_{1}, \ldots, L_{n}, M_{1}, \ldots, M_{m}$ comutam entre si.

Finalmente para mostrar $e$ ). Se $f \in C^{1}$, temos que $d f \in \mathbb{C} T^{*} U$, então

$$
d f=\sum_{j=1}^{n} \alpha_{j} d t_{j}+\sum_{k=1}^{m} \beta_{k} d Z_{k}
$$

mas

$$
\begin{gathered}
d f\left(L_{\nu}\right)=L_{\nu}(f)=\sum_{j=1}^{n} \alpha_{j} d t_{j}\left(L_{\nu}\right)+\sum_{k=1}^{m} \beta_{k} d Z_{k}\left(L_{\nu}\right)=\alpha_{\nu} \\
d f\left(M_{\nu}\right)=M_{\nu}(f)=\sum_{j=1}^{n} \alpha_{j} d t_{j}\left(M_{\nu}\right)+\sum_{k=1}^{m} \beta_{k} d Z_{k}\left(M_{\nu}\right)=\beta_{\nu} .
\end{gathered}
$$

Portanto temos que

$$
d f=\sum_{j=1}^{n} L_{j} f d t_{j}+\sum_{k=1}^{m} M_{k} f d Z_{k}
$$


Observação 3.81. Se na aplicação anterior, nós consideramos apenas um campo vetorial $L$ localmente integrável definido em um subconjunto aberto de $\mathbb{R}^{n+1}$ que contém a origem, então usando (3.6) e (3.7) podemos considerar que L tem a forma seguinte

$$
L=\frac{\partial}{\partial t}+\sum_{k=1}^{n} \lambda_{k}(x, t) \frac{\partial}{\partial x_{k}}
$$

onde cada $\lambda_{k}$ é dado por

$$
\lambda_{k}(x, t)=-i \sum_{l=1}^{n} M_{k l}(x, t) \frac{\partial \varphi_{l}}{\partial t}(x, t) .
$$

\subsection{Teorema de aproximação de Baouendi-Treves}

Nesta seção vamos discutir um dos resultados importantes na teoria das estruturas localmente integráveis, o chamado Teorema de aproximação de Baouendi-Treves, bem como algumas de suas consequências. Neste trabalho somente vamos mostrar uma versão particular deste teorema que é o caso em que temos um campo $L$ definido em um subconjunto aberto do $\mathbb{R}^{2}$ e uma função suave $Z$.

Vamos começar enunciando a versão geral do teorema, para isso precisamos da seguinte definição. Seja $\mathcal{L}$ uma estrutura formalmente integrável sobre uma variedade suave $\Omega$ de dimensão $N$.

Definição 3.82. Se $W \subset \Omega$ um subconjunto aberto e se $u \in \mathcal{D}^{\prime}(W)$ é uma distribuição sobre $W$, dizemos que u é uma solução homogênea de $\mathcal{L}$ e escrevemos $\mathcal{L} u=0$, se

$$
\text { Lu }=0 \quad \text { sobre } U
$$

para toda seção local $L$ de $\mathcal{L}$ definida sobre o subconjunto aberto $U \subset W$.

Teorema 3.83. (Teorema de aproximação de Baouendi-Treves)

Suponha que $\mathcal{L}$ é uma estrutura localmente integrável sobre uma variedade suave $\Omega$ de dimensão $N$ e que sobre o aberto $W \subset \Omega$ existam funções de classe $C^{\infty}, Z_{1}, \ldots, Z_{m}$ tais que $d Z_{1}, \ldots, d Z_{m}$ geram $\mathcal{L}^{\perp}$ sobre $W$. Seja $p \in W$, então existe uma vizinhança $U \subset W$ de $p$ tal que

i) toda $u \in \mathcal{D}^{\prime}(W)$ que satisfaz $\mathcal{L} u=0$ em $W$ é o limite em $\mathcal{D}^{\prime}(U)$ de uma sequência de polinômios $\left\{P_{j}\left(Z_{1}, \ldots, Z_{m}\right)\right\}$, ou seja,

$$
u=\lim _{j \rightarrow \infty} P_{j}\left(Z_{1}, \ldots, Z_{m}\right) \quad e m \mathcal{D}^{\prime}(U)
$$


ii) se $u \in C^{k}(W)$, a convergência ocorre na topologia de $C^{k}(U), k=0,1, \ldots$.

Demonstração. Para a demonstração deste teorema pode-se consultar [1] ou [3].

Observação 3.84. Desde que a fórmula de aproximação é de natureza local, será suficiente restringir nossa atenção a uma estrutura localmente integrável $\mathcal{L}$ definida em um subconjunto aberto $\Omega$ de $\mathbb{R}^{N}$ sobre o qual $\mathcal{L}^{\perp}$ é gerado pelos diferenciais $d Z_{1}, \ldots, d Z_{m}$ das funções $Z_{j} \in C^{\infty}(\Omega), j=1, \ldots, m$, em todo ponto de $\Omega$. Lembramos que se $n$ é a dimensão de $\mathcal{L}$, então $N=n+m$.

Exemplo 3.85. Se $\mathcal{L}$ é a estrutura localmente integrável gerada sobre um subconjunto aberto $\Omega \subset \mathbb{C}$ pelo operador de Cauchy-Riemann

$$
\bar{\partial}=\frac{1}{2}\left(\frac{\partial}{\partial x}+i \frac{\partial}{\partial y}\right), \quad z=x+i y
$$

então a distribuição solução de $\bar{\partial} u=0$ é uma função holomorfa e o teorema simplesmente afirma que, qualquer função holomorfa pode ser localmente aproximada por polinômios na variável complexa $z$.

Lema 3.86. Seja $\alpha \in \mathbb{C}, \alpha \neq 0$, então $\int_{\mathbb{R}} e^{-(\alpha x)^{2}} \alpha d x=\pi^{\frac{1}{2}}$.

Demonstração. Fazendo a mudança de variável $y=\alpha x$ temos que

$$
\int_{\mathbb{R}} e^{-(\alpha x)^{2}} \alpha d x=\int_{\mathbb{R}} e^{-y^{2}} d y
$$

Agora se $I=\int_{\mathbb{R}} e^{-y^{2}} d y$, então

$$
I^{2}=\left(\int_{\mathbb{R}} e^{-y^{2}} d y\right)\left(\int_{\mathbb{R}} e^{-z^{2}} d z\right)=\int_{\mathbb{R}^{2}} e^{-y^{2}-z^{2}} d y d z
$$

tomando $y=r \cos \theta$ e $z=r \sin \theta$, vem que

$$
I^{2}=\int_{0}^{2 \pi} \int_{0}^{+\infty} e^{-r^{2}} r d r d \theta=\int_{0}^{2 \pi} \frac{d \theta}{2}=\pi
$$

por tanto $I=\pi^{\frac{1}{2}}$.

Seja $L$ um campo localmente integrável definido em um subconjunto aberto de $\mathbb{R}^{2}$ que contém a origem. Usando o Corolário 3.79, podemos assumir que existe uma função $Z(x, t)$ definida em uma vizinhança da origem, digamos $U=(-a, a) \times(-a, a)$ tal que

$$
Z(x, t)=x+i \varphi(x, t)
$$


$\operatorname{com} \varphi$ uma função suave a valores reais, satisfazendo

$$
\varphi(0,0)=\varphi_{x}(0,0)=0
$$

Além disso, contraindo a vizinhança $U$ se for necessário podemos assumir que

$$
\left|\varphi_{x}(x, t)\right| \leq \frac{1}{2}, \quad \forall(x, t) \in U
$$

Considere agora a sequência de operadores seguinte

$$
\begin{gathered}
G_{\tau}: C^{0}(\bar{U}) \longrightarrow C^{0}(\mathbb{C} \times[-a, a]) \quad \text { dada por } \\
G_{\tau} f(z, t)=\left(\frac{\tau}{\pi}\right)^{\frac{1}{2}} \int_{-a}^{a} e^{-\tau\left[z-Z\left(x^{\prime}, t\right)\right]^{2}} f\left(x^{\prime}, t\right) Z_{x}\left(x^{\prime}, t\right) d x^{\prime}, \tau>0 .
\end{gathered}
$$

Definimos então

$$
G_{\tau} f(x, t) \doteq G_{\tau} f(Z(x, t), t),
$$

onde $Z(x, t)$ é dada em (3.8).

Teorema 3.87. (Versão do teorema de Stone Weierstrass)

Suponha que $f \in C^{0}((-a, a) \times(-a, a))$ com supp $(f) \subseteq K \times(-a, a)$, onde $K \subset(-a, a)$ é compacto, então $G_{\tau} f(x, t) \rightarrow f$ uniformemente em $\bar{U}$, quando $\tau \rightarrow+\infty$.

Demonstração. Escrevemos

$$
G_{\tau} f(x, t)=\left(\frac{\tau}{\pi}\right)^{\frac{1}{2}} \int_{-a}^{a} e^{-\tau\left[Z(x, t)-Z\left(x^{\prime}, t\right)\right]^{2}} v\left(x^{\prime}, t\right) d x^{\prime}
$$

onde $v\left(x^{\prime}, t\right)=f\left(x^{\prime}, t\right) Z_{x}\left(x^{\prime}, t\right)$. Em seguida fazendo a mudança de variável $y=\sqrt{\tau}\left(x^{\prime}-x\right)$ na integral acima obtemos

$$
G_{\tau} f(x, t)=\frac{1}{\pi^{\frac{1}{2}}} \int_{\mathbb{R}} e^{-\tau\left[Z(x, t)-Z\left(x+\frac{y}{\sqrt{\tau}}, t\right)\right]^{2}} v\left(x+\frac{y}{\sqrt{\tau}}, t\right) d y
$$

Por outro lado pelo Lema 3.86 temos

$$
f(x, t)=\frac{1}{\pi^{\frac{1}{2}}} \int_{\mathbb{R}} e^{-\left[Z_{x}(x, t) y\right]^{2}} v(x, t) d y
$$

Logo,

$$
G_{\tau} f(x, t)-f(x, t)=\frac{1}{\pi^{\frac{1}{2}}}\left(\int_{\mathbb{R}}\left[e^{-\tau\left[Z(x, t)-Z\left(x+\frac{y}{\sqrt{\tau}}, t\right)\right]^{2}} v\left(x+\frac{y}{\sqrt{\tau}}, t\right)-e^{-\left[Z_{x}(x, t) y\right]^{2}} v(x, t)\right] d y\right)
$$


daí somando e subtraindo $e^{-\left[Z_{x}(x, t) y\right]^{2}} v\left(x+\frac{y}{\sqrt{\tau}}, t\right)$ no integrando da integral acima obtemos

$$
G_{\tau} f(x, t)-f(x, t)=\pi^{-\frac{1}{2}}\left(J_{\tau}-I_{\tau}\right)
$$

onde

$$
\begin{aligned}
& J_{\tau}=\int_{\mathbb{R}}\left[e^{-\tau\left[Z(x, t)-Z\left(x+\frac{y}{\sqrt{\tau}}, t\right)\right]^{2}}-e^{-\left[Z_{x}(x, t) y\right]^{2}}\right] v\left(x+\frac{y}{\sqrt{\tau}}, t\right) d y \\
& I_{\tau}=\int_{\mathbb{R}} e^{-\left[Z_{x}(x, t) y\right]^{2}}\left[v\left(x+\frac{y}{\sqrt{\tau}}, t\right)+v(x, t)\right] d y
\end{aligned}
$$

Mostremos que $I_{\tau} \rightarrow 0$ quando $\tau \rightarrow+\infty$. De fato, notemos que

$$
\begin{aligned}
{\left[Z_{x}(x, t) y\right]^{2} } & =\left[\left(1+i \varphi_{x}(x, t)\right) y\right]^{2} \\
& =\left[y+i \varphi_{x}(x, t) y\right]^{2} \\
& =y^{2}-\left(\varphi_{x}(x, t) y\right)^{2}+2 i y^{2} \varphi_{x}(x, t)
\end{aligned}
$$

Como $\left|\varphi_{x}(x, t)\right| \leq \frac{1}{2}$, vem que

$$
\operatorname{Re}\left[\left(Z_{x}(x, t) y\right)^{2}\right]=y^{2}-\left(\varphi_{x}(x, t) y\right)^{2} \geq y^{2}-\frac{1}{4} y^{2}=\frac{1}{2} y^{2}
$$

Logo,

$$
\left|I_{\tau}\right| \leq \int_{\mathbb{R}} e^{-\frac{1}{2} y^{2}}\left|v\left(x+x \frac{y}{\sqrt{\tau}}, t\right)-v(x, t)\right| d y .
$$

Tomando $M=\sup _{\bar{U}}|v|$ e $R>0$ temos que

$$
\left|I_{\tau}\right| \leq 2 M \int_{|y| \geq R} e^{-\frac{1}{2} y^{2}} d y+\int_{|y|<R} e^{-\frac{1}{2} y^{2}}\left|v\left(x+\frac{y}{\sqrt{\tau}}, t\right)-v(x, t)\right| d y .
$$

Agora dado $\epsilon>0$, considere $R>0$ tal que $\int_{|y| \geq R} e^{-\frac{1}{2} y^{2}} d y<\frac{\epsilon}{4 M}$, o qual implica que $2 M \int_{|y| \geq R} e^{-\frac{1}{2} y^{2}} d y \leq \frac{\epsilon}{2}$.

Como $v(., t)$ é uniformemente contínua, existe $\tau_{0}>0$ tal que se $\tau \geq \tau_{0}$, então

$$
\left|v\left(x+\frac{y}{\sqrt{\tau}}, t\right)-v(x, t)\right| \leq \frac{\epsilon}{2 C}, \text { para todo }(x, t) \in \bar{U} \text { e }|y|<R
$$


onde $C=\int_{|y| \leq R} e^{-\frac{1}{2} y^{2}} d y$, daí segue que

$$
\int_{|y|<R} e^{-\frac{1}{2} y^{2}}\left|v\left(x+\frac{y}{\sqrt{\tau}}, t\right)-v(x, t)\right| d y \leq \frac{\epsilon}{2} .
$$

Portanto, se $\tau \geq \tau_{0}$ temos

$$
\left|I_{\tau}\right|<\epsilon, \text { para todo }(x, t) \in \bar{U} \text {. }
$$

Vamos estimar agora $\left|J_{\tau}\right|$. Para isto, escrevemos $J_{\tau}=J_{\tau}^{\prime}+J_{\tau}^{\prime \prime}$, onde

$$
\begin{aligned}
& J_{\tau}^{\prime}=\int_{|y| \leq R}\left[e^{-\tau\left[Z(x, t)-Z\left(x+\frac{y}{\sqrt{\tau}}, t\right)\right]^{2}}-e^{-\left[z_{x}(x, t) y\right]^{2}}\right] v\left(x+\frac{y}{\sqrt{\tau}}, t\right) d y \\
& J_{\tau}^{\prime \prime}=\int_{|y|>R}\left[e^{-\tau\left[Z(x, t)-Z\left(x+\frac{y}{\sqrt{\tau}}, t\right)\right]^{2}}-e^{-\left[Z_{x}(x, t) y\right]^{2}}\right] v\left(x+\frac{y}{\sqrt{\tau}}, t\right) d y,
\end{aligned}
$$

já vimos que $\operatorname{Re}\left[\left(Z_{x}(x, t) y\right)^{2}\right] \geq \frac{1}{2} y^{2}$ e também observemos o seguinte

$$
\begin{aligned}
\operatorname{Re}\left[Z(x, t)-Z\left(x+\frac{y}{\sqrt{\tau}}, t\right)\right]^{2} & =\operatorname{Re}\left[i \varphi(x, t)-\frac{y}{\sqrt{\tau}}-i \varphi\left(x+\frac{y}{\sqrt{\tau}}, t\right)\right]^{2} \\
& =\left(\frac{y}{\sqrt{\tau}}\right)^{2}-\left[\varphi(x, t)-\varphi\left(x+\frac{y}{\sqrt{\tau}}, t\right)\right]^{2} \\
& \geq \frac{y^{2}}{\tau}-\frac{1}{4} \frac{y^{2}}{\tau}=\frac{3}{4} \frac{y^{2}}{\tau} \geq \frac{1}{2} \frac{y^{2}}{\tau},
\end{aligned}
$$

então

$$
\left|e^{-\tau\left[Z(x, t)-Z\left(x+\frac{y}{\sqrt{\tau}}, t\right)\right]^{2}}\right|=e^{-\tau \operatorname{Re}\left(Z(x, t)-Z\left(x+\frac{y}{\sqrt{\tau}}, t\right)\right)^{2}} \leq e^{-\frac{1}{2} y^{2}} .
$$

Portanto

$$
\left|J_{\tau}^{\prime \prime}\right| \leq \int_{|y| \geq R} 2 e^{-\frac{1}{2} y^{2}} d y
$$

Dado $\epsilon>0$ tome $R>0$ tal que $2 \int_{|y| \geq R} e^{-\frac{1}{2} y^{2}} d y \leq \frac{\epsilon}{2 M}$, vem que

$$
\left|J_{\tau}^{\prime \prime}\right| \leq \frac{\epsilon}{2}, \quad \text { para todo }(x, t) \in \bar{U}
$$

Por outro lado, notemos que se $A \subset \mathbb{C}$ aberto e limitado, pela desigualdade do valor 
médio para $e^{-\xi^{2}}$, existe $c>0$ tal que

$$
\left|e^{-\xi_{1}^{2}}-e^{-\xi_{2}^{2}}\right| \leq c\left|\xi_{1}-\xi_{2}\right|, \text { para todo } \xi_{1}, \xi_{2} \in A
$$

Sejam $\xi_{1}=\sqrt{\tau}\left[Z\left(x+\frac{y}{\sqrt{\tau}}, t\right)-Z(x, t)\right]$ e $\xi_{2}=Z_{x}(x, t) y$ e como $|y| \leq R$ temos que

$$
\left|\xi_{1}\right| \leq|y|+\sqrt{\tau}\left|\varphi\left(x+\frac{y}{\sqrt{\tau}}, t\right)-\varphi(x, t)\right| \leq R+\frac{\sqrt{\tau}}{2} \frac{|y|}{\sqrt{\tau}} \leq \frac{3}{2} R, \quad \text { para todo }(x, t) \in \bar{U}
$$

$\mathrm{e}$

$$
\left|\xi_{2}\right| \leq|y|+\left|\varphi_{x}(x, t)\right||y| \leq R+\frac{R}{2}=\frac{3}{2} R, \text { para todo }(x, t) \in \bar{U}
$$

daí segue que $\xi_{1}, \xi_{2} \in \overline{B\left(0, \frac{3}{2} R\right)}=A$. Logo existe $c=c(R)>0$ tal que se $|y| \leq R$ e $(x, t) \in \bar{U}$, temos

$$
\left|e^{\xi_{1}}-e^{\xi_{2}}\right| \leq c\left|\xi_{1}-\xi_{2}\right|=c(R) \sqrt{\tau}\left|\varphi\left(x+\frac{y}{\sqrt{\tau}}, t\right)-\varphi(x, t)-\varphi_{x}(x, t) \frac{y}{\sqrt{\tau}}\right| .
$$

Por outro lado, pela fórmula de Taylor obtemos

$$
\left|\varphi\left(x+\frac{y}{\sqrt{\tau}}, t\right)-\varphi(x, t)-\varphi_{x}(x, t) \frac{y}{\sqrt{\tau}}\right| \leq \tilde{c} \frac{|y|^{2}}{\tau}, \forall(x, t) \in \bar{U}, \forall \tau>0 \text { tal que } x+\frac{y}{\sqrt{\tau}} \in(-a, a) .
$$

Logo

$$
\begin{aligned}
\left|J_{\tau}^{\prime}\right| & \leq c(R) \int_{|y| \leq R} \sqrt{\tau}\left|\varphi\left(x+\frac{y}{\sqrt{\tau}}, t\right)-\varphi(x, t)-\varphi_{x}(x, t) \frac{y}{\sqrt{\tau}}\right|\left|v\left(x+\frac{y}{\sqrt{\tau}}, t\right)\right| d y \\
& \leq c(R) \tilde{c} \int_{|y| \leq R} M \frac{y^{2}}{\sqrt{\tau}} d y \leq M \tilde{c}(R) \int_{|y| \leq R} \frac{R^{2}}{\sqrt{\tau}} d y .
\end{aligned}
$$

Agora escolha $\tau_{0} \geq 1$ tal que, se $\tau \geq \tau_{0}$ então $M \tilde{c}(R) \frac{R^{2}}{\sqrt{\tau}}<\frac{\epsilon}{4 R}$ e, portanto,

$$
\left|J_{\tau}^{\prime}\right|<\frac{\epsilon}{2}
$$

Logo de (3.11), (3.12) e (3.13) segue o resultado.

Como tínhamos falado no início da seção, vamos mostrar uma versão particular do Teorema de aproximação, neste caso consideramos como antes um campo localmente integrável $L$ definido em um subconjunto aberto de $\mathbb{R}^{2}, Z$ uma função suave e, além disso, a função $u$ é somente contínua. Logo o Teorema de aproximação pode ser enunciado da seguinte forma. 
Teorema 3.88. Seja $L=\frac{\partial}{\partial t}-\frac{Z_{t}}{Z_{x}} \frac{\partial}{\partial x}$ um campo vetorial e $Z(x, t)=x+i \varphi(x, t)$ uma função suave definidos em $U=(-a, a) \times(-a, a)$, com $\varphi \in C^{\infty}(U)$ a valores reais satisfazendo $\varphi(0,0)=0, \varphi_{x}(0,0)=0 e\left|\varphi_{x}(x, t)\right|<\frac{1}{2}$, para todo $(x, t) \in U$. Dado $p \in U$, existem dois abertos $U_{1}, U_{2}$ com $p \in U_{1} \subset \bar{U}_{1} \subset U_{2} \subset U$ tal que se $u \in C^{0}\left(U_{2}\right)$ satisfaz $L u=0$ em $U_{2}$ no sentido das distribuições, então existe uma sequência de soluções polinomiais $P_{j}(Z), j=1,2, \ldots$ tal que $P_{j}(Z) \rightarrow u$ uniformemente em $\bar{U}_{1}$.

Demonstração. Inicialmente suponhamos que $u \in C_{c}^{1}(U)$. Seja $h \in C_{c}^{\infty}(-a, a)$ tal que $h \equiv 1$ em $\left(-a^{\prime}, a^{\prime}\right), \frac{a}{2}<a^{\prime}<a$, consideremos

$$
G_{\tau} u(x, t)=\left(\frac{\tau}{\pi}\right)^{\frac{1}{2}} \int_{\mathbb{R}} e^{-\tau\left[Z(x, t)-Z\left(x^{\prime}, t\right)\right]^{2}} u\left(x^{\prime}, t\right) h\left(x^{\prime}\right) Z_{x}\left(x^{\prime}, t\right) d x^{\prime} .
$$

Vem do Teorema de Stone Weierstrass (Teorema 3.87) que $G_{\tau} u(x, t) \rightarrow u(x, t)$ uniformemente em $\left(-\frac{a}{2}, \frac{a}{2}\right) \times(-a, a)=U_{2}$.

Vamos considerar agora o operador seguinte

$$
E_{\tau} u(x, t)=\left(\frac{\tau}{\pi}\right)^{\frac{1}{2}} \int_{\mathbb{R}} e^{-\tau\left[Z(x, t)-Z\left(x^{\prime}, 0\right)\right]^{2}} u\left(x^{\prime}, 0\right) h\left(x^{\prime}\right) Z_{x}\left(x^{\prime}, 0\right) d x^{\prime}
$$

e a 1-forma dada por

$$
\omega\left(x^{\prime}, t^{\prime}\right)=\left(\frac{\tau}{\pi}\right)^{\frac{1}{2}} e^{-\tau\left[Z(x, t)-Z\left(x^{\prime}, t^{\prime}\right)\right]^{2}} u\left(x^{\prime}, t^{\prime}\right) h\left(x^{\prime}\right) d Z .
$$

Daí podemos escrever

$$
G_{\tau} u(x, t)=\int_{\mathbb{R} \times\{t\}} \omega \quad \text { e } \quad E_{\tau} u(x, t)=\int_{\mathbb{R} \times\{0\}} \omega .
$$

Pelo Teorema de Stokes vem que

$$
G_{\tau} u(x, t)-E_{\tau} u(x, t)=\int_{\mathbb{R} \times[0, t]} d \omega .
$$

Agora notemos que se $v=e^{-\tau\left[Z(x, t)-Z\left(x^{\prime}, t^{\prime}\right)\right]^{2}} u\left(x^{\prime}, t^{\prime}\right) h\left(x^{\prime}\right) d Z$, então

$$
d \omega\left(x^{\prime}, t^{\prime}\right)=\left(\frac{\tau}{\pi}\right)^{2} d(v d Z)=d v \wedge d Z=(L v d t+M v d Z) \wedge d Z=L v d t \wedge d Z
$$

onde $M=\frac{1}{1+i \varphi_{x}} \frac{\partial}{\partial x}$. Como o fator exponencial em $v$ é uma função inteira, $L Z=0$ e 
$L u=0$ em $U_{2}$, obtemos que $G_{\tau} u(x, t)-E_{\tau} u(x, t)=R_{\tau} u(x, t)$, onde

$$
R_{\tau} u(x, t)=\left(\frac{\tau}{\pi}\right)^{\frac{1}{2}} \int_{\mathbb{R} \times[0, t]} e^{-\tau\left[Z(x, t)-Z\left(x^{\prime}, t^{\prime}\right)\right]^{2}} u\left(x^{\prime}, t^{\prime}\right) L(h)\left(x^{\prime}, t^{\prime}\right) d t \wedge d Z .
$$

Provemos que $R_{\tau} u \rightarrow 0$ uniformemente em $\left(-\frac{a}{4}, \frac{a}{4}\right) \times(-T, T)$ quando $\tau \rightarrow+\infty$ e $T<a$, para $T$ escolhido convenientemente.

Notemos que

$\operatorname{Re}\left[Z(x, t)-Z\left(x^{\prime}, t^{\prime}\right)\right]^{2}=R e\left[x-x^{\prime}+i\left(\varphi(x, t)-\varphi\left(x^{\prime}, t^{\prime}\right)\right)\right]^{2}=\left|x-x^{\prime}\right|^{2}-\left|\varphi(x, t)-\varphi\left(x^{\prime}, t^{\prime}\right)\right|^{2}$.

Assim, temos o seguinte

$$
\left|e^{-\tau\left[Z(x, t)-Z\left(x^{\prime}, t^{\prime}\right)\right]^{2}}\right|=e^{\tau\left(\left|\varphi(x, t)-\varphi\left(x^{\prime}, t^{\prime}\right)\right|^{2}-\left|x-x^{\prime}\right|^{2}\right)} .
$$

Por outro lado, existe $c=c(\varphi)>0$ tal que $\left|\varphi\left(x^{\prime}, t\right)-\varphi\left(x^{\prime}, t^{\prime}\right)\right|<c\left|t-t^{\prime}\right|$ e daí

$$
\begin{aligned}
\left|\varphi(x, t)-\varphi\left(x^{\prime}, t^{\prime}\right)\right| & \leq\left|\varphi(x, t)-\varphi\left(x^{\prime}, t\right)\right|+\left|\varphi\left(x^{\prime}, t\right)-\varphi\left(x^{\prime}, t^{\prime}\right)\right| \\
& \leq \frac{1}{2}\left|x-x^{\prime}\right|+c\left|t-t^{\prime}\right| \leq \frac{1}{2}\left|x-x^{\prime}\right|+2 c T
\end{aligned}
$$

A última desigualdade acima se satisfaz por que $t \in[0, t]$ e $\left|t^{\prime}-t\right| \leq\left|t^{\prime}\right|+|t| \leq 2 T$. Logo temos

$$
\left|\varphi(x, t)-\varphi\left(x^{\prime}, t^{\prime}\right)\right|^{2} \leq 2\left(\frac{\left|x-x^{\prime}\right|^{2}}{4}+4 c^{2} T^{2}\right)=\frac{\left|x-x^{\prime}\right|^{2}}{2}+8 c^{2} T^{2} .
$$

Como $L h \equiv 0$ para $\left|x^{\prime}\right| \leq \frac{a}{2}$, notemos que no integrando da expressão (3.14) temos que $\left|x^{\prime}\right|>\frac{a}{2}$. Daí $\left|x-x^{\prime}\right| \geq\left|x^{\prime}\right|-|x| \geq \frac{a}{2}-\frac{a}{4}=\frac{a}{4}$, o qual implica que

$$
\left|R_{\tau} u(x, t)\right| \leq c e^{\tau\left(8 c^{2} T^{2}-\frac{a^{2}}{32}\right)}, \text { para todo }(x, t) \in\left(-\frac{a}{4}, \frac{a}{4}\right) \times(-T, T) .
$$

Agora escolha $T$ suficientemente pequeno tal que $8 c^{2} T^{2}<\frac{a^{2}}{32 \times 33}$, o que vai implicar que

$$
\left|R_{\tau} u(x, t)\right| \leq c e^{-\tau \frac{a^{2}}{33}}, \text { para todo }(x, t) \in\left(-\frac{a}{4}, \frac{a}{4}\right) \times(-T, T)=U_{1}
$$

o que mostra que $R_{\tau} u(x, t) \rightarrow 0$, quando $\tau \rightarrow+\infty$ em $U_{1}$. Portanto, $E_{\tau} u=G_{\tau} u-R_{\tau} u \rightarrow$ $u$ uniformemente quando $\tau \rightarrow+\infty$ em $U_{1}$. 
Temos provado que $R_{\tau} u(x, t) \rightarrow 0$ uniformemente quando $\tau \rightarrow+\infty$ em $U_{1}$, se $u \in C^{0}$.

Para concluirmos a demonstração mostraremos que vale a formula $G_{\tau} u-E_{\tau} u=R_{\tau} u$ em $\mathcal{D}^{\prime}$ para $u \in C^{0}$. De fato, considere $\psi_{\epsilon}(x)=\frac{1}{\epsilon^{2}} \psi\left(\frac{x}{\epsilon}, \frac{t}{\epsilon}\right)$, com $\psi \in C_{c}^{\infty}(\Omega)$ e seja $u_{\epsilon}(x, t)=u * \psi_{\epsilon}$, temos que $u_{\epsilon} \in C^{\infty}(\Omega)$. Logo, para cada $\tau>0$ fixo aplicando o Teorema de Stokes temos

$$
G_{\tau} u_{\epsilon}(x, t)-E_{\tau} u_{\epsilon}(x, t)=R_{\tau} u_{\epsilon}(x, t)
$$

onde

$$
\begin{aligned}
& R_{\tau} u_{\epsilon}(x, t)=\int_{\mathbb{R} \times[0, t]} d \omega_{\epsilon} \quad \mathrm{e} \\
& d \omega_{\epsilon}(x, t)=\left(\frac{\tau}{\pi}\right)^{\frac{1}{2}} e^{-\tau\left[Z(x, t)-Z\left(x^{\prime}, t^{\prime}\right)\right]^{2}} u_{\epsilon}\left(x^{\prime}, t^{\prime}\right) h\left(x^{\prime}\right) d Z\left(x^{\prime}, t^{\prime}\right) .
\end{aligned}
$$

Portanto, se $v_{\epsilon}\left(x^{\prime}, t^{\prime}\right)=e^{-\tau\left[Z(x, t)-Z\left(x^{\prime}, t^{\prime}\right)\right]^{2}} u_{\epsilon}\left(x^{\prime}, t^{\prime}\right) h\left(x^{\prime}\right)$, vem que

$$
\begin{aligned}
d \omega_{\epsilon}\left(x^{\prime}, t^{\prime}\right)= & d v_{\epsilon}\left(x^{\prime}, t^{\prime}\right) \wedge d Z\left(x^{\prime}, t^{\prime}\right)=\left[L v_{\epsilon} d t+M v_{\epsilon} d Z\right] \wedge d Z \\
= & e^{-\tau\left[Z(x, t)-Z\left(x^{\prime}, t^{\prime}\right)\right]^{2}} L u_{\epsilon}\left(x^{\prime}, t^{\prime}\right) h\left(x^{\prime}\right) d t \wedge d Z\left(x^{\prime}, t^{\prime}\right) \\
& +e^{-\tau\left[Z(x, t)-Z\left(x^{\prime}, t^{\prime}\right)\right]^{2}} u_{\epsilon}\left(x^{\prime}, t^{\prime}\right) L h\left(x^{\prime}\right) d t \wedge d Z\left(x^{\prime}, t^{\prime}\right)
\end{aligned}
$$

e como $u_{\epsilon} \rightarrow u$ uniformemente em $\Omega$ quando $\epsilon \rightarrow 0$ e $L u_{\epsilon} \rightarrow L u$ em $\mathcal{D}^{\prime}(\Omega)$, obtemos que

$$
R_{\tau} u_{\epsilon} \rightarrow R_{\tau} u ; G_{\tau} u_{\epsilon} \rightarrow G_{\tau} u \text { e } E_{\tau} u_{\epsilon} \rightarrow E_{\tau} u \text { em } \mathcal{D}^{\prime}(\Omega)
$$

Portanto

$$
G_{\tau} u-E_{\tau} u=R_{\tau} u \text { em } \mathcal{D}^{\prime}(\Omega), \text { para todo } \tau>0 \text {, e para todo } u \in C^{0} \text {. }
$$

Por outro lado, como $R_{\tau} u \rightarrow 0$ e $G_{\tau} u \rightarrow u$ uniformemente em $U_{1}$ quando $\tau \rightarrow+\infty$, temos que $E_{\tau} u \rightarrow u$ uniformemente em $U_{1}$ quando $\tau \rightarrow+\infty$.

Agora sejam

$$
F_{j}(z)=\left(\frac{j}{\pi}\right)^{\frac{1}{2}} \int_{\mathbb{R}} e^{-\tau\left[z-Z\left(x^{\prime}, 0\right)\right]^{2}} u\left(x^{\prime}, 0\right) h\left(x^{\prime}\right) Z_{x}\left(x^{\prime}, t\right) d x^{\prime} .
$$

Note que $F_{j}$ são funções inteiras em $\mathbb{C}$. Logo para cada $j$ existe um polinômio $P_{j}(z)$ tal que

$$
\left\|P_{j}-F_{j}\right\|_{C^{0}(\{z ;|z| \leq R\})}<\frac{1}{j}
$$


onde $R$ é escolhido de maneira que $Z\left(U_{1}\right) \subset\{z ;|z| \leq R\}$. Daí temos que

$\left\|f-P_{j} \circ Z\right\|_{C^{0}(\bar{U})} \leq\left\|f-F_{j} \circ Z\right\|_{C^{0}\left(\bar{U}_{1}\right)}+\left\|F_{j} \circ Z-P_{j} \circ Z\right\|_{C^{0}\left(\bar{U}_{1}\right)} \longrightarrow 0$ quando $j \rightarrow \infty$

o qual prova o teorema.

Corolário 3.89. Nas mesmas hipóteses que no Teorema de aproximação de BaouendiTreves, existe $U_{0}$ vizinhança de $p$ tal que se $u \in C^{0}(U)$ for uma solução de Lu $=0$ tem-se $u\left(p_{1}\right)=u\left(p_{2}\right)$ quaisquer que sejam os pontos $p_{1}, p_{2} \in U_{0}$ satisfazendo $Z\left(p_{1}\right)=Z\left(p_{2}\right)$.

Demonstração. Como $Z\left(p_{1}\right)=Z\left(p_{2}\right)$, então $P\left(Z\left(p_{1}\right)\right)=P\left(Z\left(p_{2}\right)\right)$, ou seja, $(P \circ Z)\left(p_{1}\right)=$ $(P \circ Z)\left(p_{2}\right)$ para qualquer polinômio $P$ em uma variável complexa. Logo pelo Teorema de aproximação de Bouendi-Treves temos que

$$
u\left(p_{1}\right)=\lim _{j \rightarrow \infty} P_{j}(Z)\left(p_{1}\right)=\lim _{j \rightarrow \infty} P_{j}(Z)\left(p_{2}\right)=u\left(p_{2}\right)
$$

Corolário 3.90. Nas mesmas hipóteses que no Teorema de aproximação de BaouendiTreves, existe uma vizinhança $V_{0}$ de $p_{0}$ tal que se $u \in C^{0}(U)$ for uma solução de $L u=0$, existe $F: Z\left(V_{0}\right) \longrightarrow \mathbb{C}$ continua e holomorfa no interior de $Z\left(V_{0}\right)$, tal que $u=F \circ Z$ sobre $V_{0}$.

Demonstração. Tomemos $V_{0}$ como sendo qualquer vizinhança de $p_{0}$ com $\bar{V}_{0} \subset U_{1}$ onde $U_{1}$ é a vizinhança dada pelo Teorema de aproximação. Logo, para cada $\xi \in Z\left(\bar{V}_{0}\right)$ definimos

$$
F(\xi)=u(p) \quad \text { se } Z(p)=\xi
$$

Temos que $F$ é bem definida pelo corolário anterior. Mais ainda, $F$ é holomorfa no interior de $Z\left(V_{0}\right)$ pois é o limite uniforme de funções inteiras.

Mostremos agora que $F$ é contínua. De fato, seja $\left(\xi_{l}\right)$ uma sequência em $Z\left(\bar{V}_{0}\right)$ $\operatorname{com} \xi_{l} \rightarrow \xi^{*} \in Z\left(\bar{V}_{0}\right)$. Tomemos $\left(p_{l}\right) \subset \bar{V}_{0}$ sequência tal que $Z\left(p_{l}\right)=\xi_{l}$. Passando a uma subsequência se for necessário, podemos assumir que $p_{l} \rightarrow p^{*} \in \bar{V}_{0}$, portanto $u\left(p_{l}\right)=F\left(\xi_{l}\right) \rightarrow u\left(p^{*}\right)$, pois $u$ é contínua.

Por outro lado $\xi_{l}=Z\left(p_{l}\right) \rightarrow Z\left(p^{*}\right)$ (pois $Z$ é contínua) e $\xi_{l} \rightarrow \xi^{*}$, daí $Z\left(p^{*}\right)=\xi^{*}$ o que implica que $F\left(\xi^{*}\right)=u\left(p^{*}\right)=\lim _{l \rightarrow \infty} F\left(\xi_{l}\right)$. Portanto, $F$ é contínua.

Uma aplicação do Teorema de aproximação de Baouendi-Treves tem a ver com a propagação de zeros de soluções homogêneas. Ou seja, dada uma estrutura localmente integrável $\mathcal{L}$ em uma variedade $\Omega$ de dimensão $N$ e uma solução $u$ de $\mathcal{L} u=0$ a questão 
natural é: que condição deve satisfazer a solução $u$ para concluir que $u$ é identicamente zero?. A versão local da questão é: dado um ponto $p \in \Omega$, e uma vizinhança $V$ de $p$, que condições garantem a existência de uma vizinhança $p \in U \subset V$ sobre a qual $u$ zera? A resposta a esta questão é dada no Teorema 3.94 mas para isso precisamos da seguinte definição.

Definição 3.91. Seja $\Sigma \subset \Omega$ uma subvariedade mergulhada. Dizemos que $\Sigma$ é maximal real com respeito a $\mathcal{L}$ se

i) $\operatorname{dim} \Sigma=m$.

ii) para todo $p \in \Sigma$ e qualquer seção $L$ de $\mathcal{L}$ definida em uma vizinhança de $p$, temos que se $L=X+i Y$ então $X_{p} \notin \mathbb{C} T_{p} \Sigma$ ou $Y_{p} \notin \mathbb{C} T_{p} \Sigma$.

Resumindo temos que $\mathbb{C} T_{p} \Omega=\mathbb{C} T_{p} \Sigma \oplus \mathcal{L}_{p}, \forall p \in \Sigma$.

Proposição 3.92. Seja $\mathcal{L}$ uma estrutura localmente integrável em $\Omega$ e $\Sigma \subset \Omega$ uma subvariedade real maximal em relação a $\mathcal{L}$. Existe um sistema de coordenadas em torno da origem tal que $\mathcal{L}^{\perp}=<d Z_{1}, \ldots, d Z_{m}>\operatorname{com} Z(x, t)=\left(Z_{1}(x, t), \ldots, Z_{m}(x, t)\right), Z(x, t)=$ $x+i \varphi(x, t), \varphi(0,0)=0, d_{x} \varphi(0,0)=0$ e $\Sigma=\{(x, 0)\}$.

Demonstração. Considere coordenadas $\left(x_{1}, \ldots, x_{m}, t_{1}, \ldots, t_{n}\right)=(x, t) \operatorname{com}(x(p), t(p))=$ $(0,0)$ tal que $\mathcal{L}^{\perp}$ é gerado numa vizinhança de $(0,0)$ por $d Z_{1}, \ldots, d Z_{m}$, onde $Z_{j}(x, t)=$ $x_{j}+i \varphi_{j}(x, t), j=1, \ldots, m$, com $\varphi(0,0)=0$ e $d_{x} \varphi(0,0)=0$ onde $\varphi=\left(\varphi_{1}, \ldots, \varphi_{m}\right)$. Daí temos que $\left.\operatorname{Re} L_{j}\right|_{(0,0)}=\frac{\partial}{\partial t_{j}}$. Como $\Sigma$ é maximal real em relação a $\mathcal{L}$, vem que $\mathbb{C} T_{(0,0)} \Omega=\mathbb{C} T_{(0,0)} \Sigma \oplus<\frac{\partial}{\partial t_{1}}, \ldots, \frac{\partial}{\partial t_{n}}>$.

Considere

$$
\begin{aligned}
A=d \varphi(0,0): \mathbb{R}^{n+m} & \longrightarrow \mathbb{R}^{m} \\
(h, k) & \longmapsto A(h, k),
\end{aligned}
$$

então $A_{t}=d_{t} \varphi(0,0)$ e $A_{x}=d_{x} \varphi(0,0)$.

Vem das hipóteses que $A_{t}$ é invertível e pelo Teorema da função implícita existe $U \subset \mathbb{R}^{n+m}$ e $W \subset \mathbb{R}^{m}$ com $(0,0) \in U$ e $0 \in W$ tal que para todo $x \in W$ existe um único $t=\tau(x)$ $\operatorname{com}(x, t)=(x, \tau(x)) \in U$ e $\varphi(x, \tau(x))=0$. Além disso,

$$
\tau \in C^{1}(W), \quad \tau(0)=0 \quad \text { e } \quad \tau^{\prime}(0)=-\left(A_{t}\right)^{-1} A_{x}=-\left(d_{t} \varphi(0,0)\right)^{-1}\left(d_{x} \varphi(0,0)\right)=0 .
$$

Portanto, localmente temos que $\Sigma=\{(x, \tau(x))\}$.

Agora considere o seguinte sistema de coordenadas $x^{\prime}=x$ e $t^{\prime}=t-\tau(x)$, então a expressão de $Z$ nestas novas coordenadas é

$$
Z^{\prime}\left(x^{\prime}, t^{\prime}\right)=x^{\prime}+i \varphi\left(x^{\prime}, t^{\prime}+\tau(x)\right)=x^{\prime}+i \varphi^{\prime}\left(x^{\prime}, t^{\prime}\right)
$$


$\operatorname{com} \varphi^{\prime}(0,0)=\varphi(0,0+\tau(0))=\varphi(0,0)=0$ e pela regra da cadeia temos $d_{x^{\prime}} \varphi^{\prime}(0,0)=0$. nestas novas coordenadas temos localmente $\Sigma=\left\{\left(x^{\prime}, 0\right)\right\}$.

Observação 3.93. Uma consequência da proposição anterior é que, se u é uma distribuição solução de $\mathcal{L} u=0$ podemos sempre considerar sua restrição a $\Sigma,\left.u\right|_{\Sigma}$, que é apenas o traço de u sobre $\Sigma, u(x, 0)$.

Teorema 3.94. Seja $\mathcal{L}$ uma estrutura localmente integrável em uma variedade $\Omega$ de dimensão $N$ e $\Sigma \subset \Omega$ uma subvariedade mergulhada real maximal com respeito a $\mathcal{L}$. Se $u \in C^{0}(\Omega)$ e satisfaz
i) $\mathcal{L} u=0$ em $\Omega$.
ii) $\left.u\right|_{\Sigma}=0$.

então $u \equiv 0$ em uma vizinhança $V$ de $\Sigma$.

Demonstração. É suficiente mostrar que qualquer ponto $p \in \Sigma$ está contido numa vizinhança $U$ tal que $\left.u\right|_{U} \equiv 0$.

Pela Proposição 3.92, dado $p \in \Sigma$, existem coordenadas especiais tais que $\Sigma=\{(x, 0)\}$ e aplicamos o Teorema de aproximação de Baouendi-Treves nesta vizinhança de $p=(0,0)$, podemos encontrar abertos $U \subset W, 0 \in U$ tal que $W$ está contido na vizinhança de coordenadas iniciais e $u$ é aproximado em $U$ por $E_{\tau} u$ uniformemente quando $\tau \rightarrow+\infty$. Por outro lado, a fórmula que define $E_{\tau} u$ em $W$ é a seguinte

$$
E_{\tau} u(x, t)=\left(\frac{\tau}{\pi}\right)^{\frac{1}{2}} \int_{B_{x}(0, R)} e^{-\tau\left[Z(x, t)-Z\left(x^{\prime}, 0\right)\right]^{2}} u\left(x^{\prime}, 0\right) h\left(x^{\prime}\right) d Z_{x}\left(x^{\prime}, 0\right) d x \quad, \quad \forall(x, t) \in W .
$$

Mas $u\left(x^{\prime}, 0\right) \equiv 0$ em $\Sigma \cap W=B_{x}(0, R) \times\{0\}$, então $E_{\tau} u(x, t) \equiv 0$ em $W$ e como $E_{\tau} u(x, t) \rightarrow u$ uniformemente em $U \subset W$ quando $\tau \rightarrow+\infty$, vem que $u \equiv 0$ em $U$.

Corolário 3.95. Seja $L$ um campo não singular em $\Omega$ tal que $\mathcal{L}=<L>$ é localmente integrável e seja $u \in C^{0}(\Omega)$ satisfazendo $L u=0$ em $\Omega, X=R e L$ e $p, q$ dois pontos em $\Omega$. Assuma que $\gamma$ é uma curva integral de $X$ ligando $p$ e q. Então se $p \in \operatorname{supp}(u)$ temos $q \in \operatorname{supp}(u)$.

Demonstração. Se $X \equiv 0$ então temos que $\gamma$ é constante logo $p=q$ e não há o que provar. Assumimos então que $\gamma:[0,1] \longrightarrow \Omega$ é uma solução não constante de $\gamma^{\prime}(s)=X(\gamma(s))$, $0 \leq s \leq 1$ com $\gamma(0)=q$ e $\gamma(1)=p$. Logo $X$ não zera em uma vizinhança de $\gamma$. Denote por $K=\operatorname{supp}(u)$ e suponhamos por contradição que $p \in K$ e $q \notin K$. Trocando $p$ pelo primeiro ponto $\gamma(s)$ tal que $\gamma(s) \in K$, podemos assumir que $p$ e $q$ estão suficientemente 
próximos de modo que todos os pontos em $\gamma$ entre $p$ e $q$ não estão em $K$. Ou seja, sem perda de generalidade podemos supor que estamos na seguinte situação

$$
\left\{\begin{array}{l}
\gamma(0)=q \\
\gamma(1)=p \\
\gamma(s) \notin K, \text { para todo } 0<s<1
\end{array}\right.
$$

Por outro lado, pelo Teorema de Frobenius (Teorema 3.75), podemos encontrar coordenadas $(x, t) \in \mathbb{R}^{m+1}$ apropriadas tal que localmente $\langle L\rangle=\langle\partial / \partial t\rangle,|x|<1,|t|<2$ e $p=(0,0)$.

i) $\gamma(s)=(s-1,0, \ldots, 0) ; q=\gamma(0)=(-1,0, \ldots, 0)$.

ii) Para algum $a>0$ a $m$-bola fechada dada por $|x| \leq a$ e $t=-1$ não encontra-se em $K$. Denotando esta bola por $\bar{\Sigma}_{0}$, temos que

$$
\begin{aligned}
\bar{\Sigma}_{0} & =\{(x, t) ;|x| \leq a, t=-1\} \\
& =\{(x, t) ;|x|<a, t=-1\} \cup\{(x, t) ;|x|=a, t=-1\} \\
& =\Sigma_{0} \cup \partial \Sigma_{0} .
\end{aligned}
$$

Considere agora a família a um parâmetro de subvariedades mergulhadas

$$
\Sigma_{\sigma}=\left\{(x, t) ;|x|<a, t=\sigma-1-\sigma \frac{|x|^{2}}{a^{2}}\right\}, 0 \leq \sigma \leq 1 .
$$

De $i i)$ temos que $\Sigma_{0} \cap K=\emptyset$ e $(0,0) \in \Sigma_{1} \cap K$. Logo existe $\sigma_{0} \in(0,1]$ tal que $\Sigma_{\sigma} \cap K=\emptyset$ para $0 \leq \sigma<\sigma_{0} \mathrm{e}$

$$
\Sigma_{\sigma_{0}} \cap K \neq \emptyset
$$

Notemos que $\Sigma_{\sigma}$ são subvariedades reais maximais em relação a $\mathcal{L}=\langle L\rangle=\langle\partial / \partial t\rangle$.

Assim temos que $\left.u\right|_{\Sigma_{\sigma}}=0$ para $0<\sigma<\sigma_{0}$, (pois $\Sigma_{\sigma} \cap K=\emptyset$ ) e como a função $\left.\sigma \longmapsto u\right|_{\Sigma_{\sigma}}$ é contínua, temos que

$$
\left.u\right|_{\Sigma_{\sigma_{0}}}=0 \text {. }
$$

Logo, pelo Teorema 3.94, existe uma vizinhança de $\Sigma_{\sigma_{0}}$ tal que $u \equiv 0$ nesta vizinhança, ou seja, $\Sigma_{\sigma_{0}} \cap K=\emptyset$ o que contradiz (3.15). 


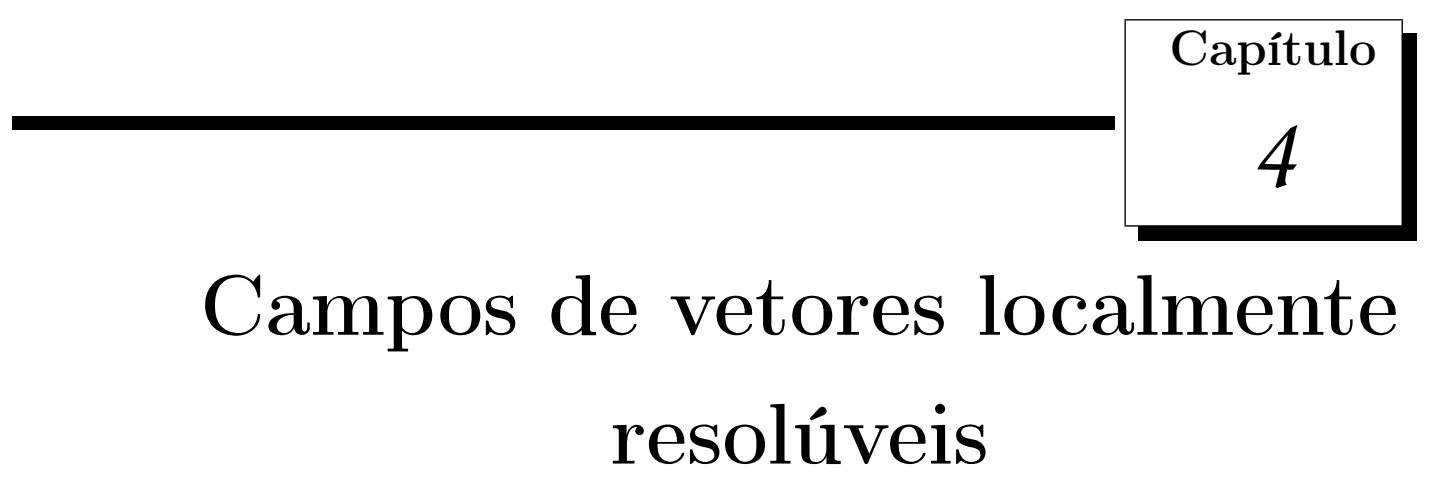

Neste capítulo vamos estudar de forma rápida uma importante classe de campos de vetores localmente integráveis, os chamados campos localmente resolúveis, além disso, introduziremos a conhecida condição (P) de Nirenberg-Treves, bem como a definição de órbitas de um campo vetorial no sentido de Sussmann. Primeiro fazemos o estudo para campos vetoriais definidos no plano e em seguida fazemos o estudo para campos vetoriais em várias variáveis. Para este capítulo nossas referências são [1], [3] e [14].

\subsection{Campos de vetores no plano}

Considere o campo definido em um aberto $\Omega \subset \mathbb{R}^{2}$

$$
L=A(x, t) \frac{\partial}{\partial t}+B(x, t) \frac{\partial}{\partial x} \quad, \quad(x, t) \in \Omega
$$

Com $A, B$ a valores complexos, $A, B \in C^{\infty}(\Omega)$ satisfazendo

$$
|A(x, t)|+|B(x, t)|>0, \text { para todo }(x, t) \in \Omega \text {. }
$$

Logo de (4.1) temos que $A$ ou $B$ nunca se anulam na vizinhança de um ponto em $\Omega$. Assim podemos supor sem perda de generalidade que $A$ não se anula em uma vizinhança de um ponto de $\Omega$ e multiplicamos $L$ por $A^{-1}$ para obter o campo $\tilde{L}=A^{-1} L$ que assume a forma seguinte

$$
\tilde{L}=\frac{\partial}{\partial t}+\tilde{B}(x, t) \frac{\partial}{\partial x}
$$


Agora escrevendo $\tilde{B}(x, t)=\tilde{a}(x, t)+i \tilde{b}(x, t)$, com $\tilde{a}, \tilde{b}$ funções a valores reais e assuma que elas estão definidas em $(-\rho, \rho) \times(-\rho, \rho) \operatorname{com} \rho>0$. Vamos dar o seguinte resultado

Lema 4.1. Existem coordenadas locais em torno da origem $\xi=x, s=s(x, t)$, onde o campo $\tilde{L}$ assume a forma seguinte

$$
\tilde{L}=\frac{\partial}{\partial s}+i b(\xi, s) \frac{\partial}{\partial \xi}
$$

$\operatorname{com} b(\xi, s)$ função suave a valores reais.

Demonstração. Considere o P.V.I de e.d.o

$$
\begin{cases}\frac{d x}{d s}=\tilde{a}(x, t) & ; x(0)=\xi \\ \frac{d t}{d s}=1 & ; t(0)=0\end{cases}
$$

com solução $(x(\xi, s), t(\xi, s))$ dada por

$$
\begin{aligned}
& x(\xi, s)=\xi+\int_{0}^{s} \tilde{a}(x(\xi, \sigma), \sigma) d \sigma \\
& t(\xi, s)=s .
\end{aligned}
$$

Observamos que $x(\xi, 0)=\xi$, logo

$$
\frac{\partial x}{\partial \xi}(\xi, 0)=1 \Longrightarrow \frac{\partial x}{\partial \xi}(0,0)=1
$$

e além disso,

$$
\frac{\partial t}{\partial \xi}(0,0)=0 \quad \text { e } \quad \frac{\partial t}{\partial s}(0,0)=1
$$

assim o determinante jacobiano é

$$
\left|\begin{array}{ll}
\frac{\partial x}{\partial \xi}(0,0) & \frac{\partial x}{\partial s}(0,0) \\
\frac{\partial t}{\partial \xi}(0,0) & \frac{\partial t}{\partial s}(0,0)
\end{array}\right|=\left|\begin{array}{cc}
1 & \frac{\partial x}{\partial s}(0,0) \\
0 & 1
\end{array}\right|=1 \neq 0
$$

garantindo que a aplicação $(\xi, s) \longrightarrow(x(\xi, s), t(\xi, s))$ é localmente uma mudança de va- 
riável. Ainda pela regra da cadeia vem que

$$
\begin{aligned}
\frac{\partial}{\partial s}=\frac{\partial}{\partial x} \frac{\partial x}{\partial s}+\frac{\partial}{\partial t} \frac{\partial t}{\partial s} & =\tilde{a}(x, t) \frac{\partial}{\partial x}+\frac{\partial}{\partial t} \\
\frac{\partial}{\partial \xi}=\frac{\partial}{\partial x} \frac{\partial x}{\partial \xi}+\frac{\partial}{\partial t} \frac{\partial t}{\partial \xi} & =\frac{\partial x}{\partial \xi} \frac{\partial}{\partial x} .
\end{aligned}
$$

Portanto, nestas coordenadas temos

$$
\begin{aligned}
\tilde{L} & =\frac{\partial}{\partial t}+(\tilde{a}(x, t)+i \tilde{b}(x, t)) \frac{\partial}{\partial x}=\frac{\partial}{\partial t}+\tilde{a}(x, t) \frac{\partial}{\partial x}+i \tilde{b}(x, t) \frac{\partial}{\partial x} \\
& =\frac{\partial}{\partial s}+i \frac{\tilde{b}(x, t)}{\frac{\partial x}{\partial \xi}} \frac{\partial}{\partial \xi}=\frac{\partial}{\partial s}+i b \frac{\partial}{\partial \xi}
\end{aligned}
$$

ou seja, $\tilde{L}$ tem a seguinte forma

$$
\tilde{L}=\frac{\partial}{\partial s}+i b \frac{\partial}{\partial \xi}
$$

As reduções descritas acima mostram que, no estudo dos problemas locais para um campo de vetores no plano $L$ com coeficientes suaves, podemos assumir sempre que $L$ é da forma

$$
L=\frac{\partial}{\partial t}+i b(x, t) \frac{\partial}{\partial x}
$$

com $b(x, t)$ função suave a valores reais definida para todo $(x, t) \in \Omega \subset \mathbb{R}^{2}$.

Vamos dar agora a noção de resolubilidade local de um campo vetorial $L$.

Definição 4.2. Seja L um campo vetorial definido em um aberto $\Omega \subset \mathbb{R}^{2}, p \in \Omega$. Dizemos que $L$ é localmente resolúvel em $p$ se existe uma vizinhança $U=U(p)$ tal que para toda $f \in C^{\infty}(\Omega)$ existe $u \in \mathcal{D}^{\prime}(\Omega)$ tal que Lu $=f$ em $U$.

Se $L$ é localmente resolúvel em todo ponto $p \in \Omega$, dizemos que $L$ é localmente resolúvel em $\Omega$.

Observação 4.3. Se na definição anterior a função u pertence a $C^{\infty}(U)$, dizemos que o campo L é localmente resolúvel em $C^{\infty}$.

Teorema 4.4. Se L é localmente resolúvel em $C^{\infty}$, então L é localmente integrável.

Demonstração. Dado um ponto $p \in \Omega$, que podemos assumir que é a origem, gostaríamos de encontrar uma função suave $Z$, definida em uma vizinhança da origem, tal que nesta 
vizinhança $L Z=0$ e $d Z \neq 0$.

Seja $d(x, t)=i \frac{\partial b}{\partial x}(x, t)$, então existe $u \in C^{\infty}(\Omega)$ tal que $L u=d$ em um retângulo $U$ centrado na origem.

Considere a 1-forma seguinte

$$
\omega=i b(x, t) e^{-u} d t+e^{-u} d x
$$

Mostraremos que $\omega$ é fechada. De fato

$$
\begin{aligned}
d \omega & =d\left(i b e^{-u}\right) \wedge d t+d\left(e^{-u}\right) \wedge d x \\
& =\left(\frac{\partial}{\partial x}\left(i b e^{-u}\right) d x+\frac{\partial}{\partial t}\left(i b e^{-u}\right) d t\right) \wedge d t+\left(\frac{\partial}{\partial x} e^{-u} d x+\frac{\partial}{\partial t} e^{-u} d t\right) \wedge d x \\
& =\frac{\partial}{\partial x}\left(i b e^{-u}\right) d x \wedge d t+\left(-\frac{\partial}{\partial t} e^{-u}\right) d x \wedge d t \\
& =\left[\frac{\partial}{\partial x}\left(i b e^{-u}\right)-\frac{\partial}{\partial t}\left(e^{-u}\right)\right] d x \wedge d t,
\end{aligned}
$$

e, por outro lado, temos que

$$
\frac{\partial}{\partial x}\left(i b e^{-u}\right)-\frac{\partial}{\partial t}\left(e^{-u}\right)=i \frac{\partial b}{\partial x} e^{-u}-i b e^{-u} \frac{\partial u}{\partial x}+e^{-u} \frac{\partial u}{\partial t}=e^{-u}\left(i \frac{\partial b}{\partial x}-L u\right)=0 .
$$

Logo de (4.4) e (4.5) concluímos que $d \omega=0$, o qual implica que $\omega$ é fechada.

Daí como $U$ é simplesmente conexo temos que $\omega$ é exata, ou seja, existe $Z \in C^{\infty}(U)$ tal que $d Z=\omega$. Então $d Z \neq 0$ em $U$ e também

$$
L Z=d Z(L)=\omega(L)=e^{-u}(i b d t-d x)\left(\frac{\partial}{\partial t}+i b \frac{\partial}{\partial x}\right)=e^{-u}(i b-i b)=0
$$

Portanto, temos que existe $Z \in C^{\infty}(U)$ tal que $d Z \neq 0$ e $L Z=0$. Isto é, $L$ é localmente integrável.

\section{Observação 4.5.}

a) Como $d Z=\omega$ então $Z_{x} \neq 0$ em $U$ pois $Z_{x} d x+Z_{t} d t=-e^{-u} d x+i b e^{-u} d t$ e $e^{-u} \neq 0$. Logo, como $Z=\operatorname{Re} Z+i \operatorname{Im} Z$ e $Z_{x}=\operatorname{Re} Z_{x}+i \operatorname{Im} Z_{x} \neq 0$ em $U$, podemos supor que $\operatorname{Re} Z_{x} \neq 0$ em $U$.

Considere a seguinte mudança de coordenadas $\tilde{x}=R e Z$ e $\tilde{t}=t$, obtemos que

$$
\frac{\partial(\tilde{x}, \tilde{t})}{\partial(x, t)}=\left|\begin{array}{cc}
\operatorname{Re} Z_{x} & \operatorname{Re} Z_{t} \\
0 & 1
\end{array}\right|=\operatorname{Re} Z_{x} \neq 0 \quad \text { em } U
$$


Nestas novas coordenadas temos que

$$
Z=\tilde{x}+i \varphi(\tilde{x}, \tilde{t})
$$

Logo

$$
L=L(\tilde{t}) \frac{\partial}{\partial \tilde{t}}+L(\tilde{x}) \frac{\partial}{\partial \tilde{x}}=\frac{\partial}{\partial t}+a(x, t) \frac{\partial}{\partial x},
$$

com a $(x, t)$ função complexa. Além disso temos que

$$
L Z=0 \quad \Longleftrightarrow \quad Z_{t}+a(x, t) Z_{x}=0 \quad \Longleftrightarrow \quad a(x, t)=-\frac{Z_{t}}{Z_{x}}
$$

portanto obtemos

$$
L=\frac{\partial}{\partial t}-\frac{Z_{t}}{Z_{x}} \frac{\partial}{\partial x}
$$

b) Suponha que $\varphi_{t} \neq 0$ em $U$ ( $L$ é elítico), $Z(x, t)=x+i \varphi(x, t)$ e considere $y=x$, $s=\varphi(x, t)$. Logo temos que

$$
\frac{\partial(y, s)}{\partial(x, t)}=\left|\begin{array}{cc}
1 & 0 \\
\varphi_{x} & \varphi_{t}
\end{array}\right|=\varphi_{t} \neq 0 \quad \text { em } U
$$

Segue-se que a aplicação $(x, t) \longrightarrow(y, s)$ é uma mudança de coordenadas em $U$ e temos que

$$
Z=y+i s \quad e \quad L=L(y) \frac{\partial}{\partial y}+L(s) \frac{\partial}{\partial s} .
$$

Como $L(Z)=0$ então $L(y+i s)=0$, o qual implica que $L(y)=-i L(s)$. Portanto, $L$ fica da seguinte forma

$$
L=-i L s \frac{\partial}{\partial y}+L s \frac{\partial}{\partial s}=2 L s(-i) \frac{1}{2}\left(\frac{\partial}{\partial y}+i \frac{\partial}{\partial s}\right)=g \frac{\partial}{\partial \bar{z}} .
$$

Definição 4.6. Dizemos que o operador $L$ dado por (4.3) satisfaz a condição (P) em $p_{0}=\left(x_{0}, t_{0}\right)$ se existe uma vizinhança $\left(x_{0}-\delta, x_{0}+\delta\right) \times\left(t_{0}-\delta, t_{0}+\delta\right)$ em $p_{0}$ tal que para todo $x \in\left(x_{0}-\delta, x_{0}+\delta\right)$ a função $\left(t_{0}-\delta, t_{0}+\delta\right) \ni t \longmapsto b(x, t)$ não muda de sinal.

Exemplo 4.7. Considere o campo vetorial em $\mathbb{R}^{2}$ dado por $L=\frac{\partial}{\partial t}-i t \frac{\partial}{\partial x}$. Neste caso $b(x, t)=b(t)=-t$.

Se $p=(x, 0)$, então a função $(-\delta, \delta) \ni t \longmapsto b(t)=-t$ muda de sinal. Portanto, $L$ não satisfaz a condição $(\mathrm{P})$ nos pontos da forma $p=(x, 0)$.

Se $p=(x, y)$, com $y \neq 0$, temos $(-\delta, \delta) \ni t \longmapsto b(t)=-t$ não muda de sinal. Portanto $L$ satisfaz a condição $(\mathrm{P})$ nos pontos da forma $(x, y)$ com $y \neq 0$. 
Exemplo 4.8. Considere o campo vetorial definido por

$$
L=\frac{\partial}{\partial t}+i x \frac{\partial}{\partial x}
$$

neste caso $b(x, t)=b(x)=x$. Logo, temos que $L$ satisfaz a condição $(\mathrm{P})$ em todo $\mathbb{R}^{2}$.

Exemplo 4.9. Seja o campo vetorial

$$
L=\frac{\partial}{\partial t}-i x t^{2} \frac{\partial}{\partial x}
$$

aqui temos que $b(x, t)=-x t^{2} \operatorname{Logo}, L$ satisfaz a condição $(\mathrm{P})$ em todo $\mathbb{R}^{2}$.

Na Definição 4.6 assumimos que o campo vetorial $L$ estava na forma especial (4.3), no entanto exigir que a função $t \longmapsto b(x, t)$ não mude de sinal é uma condição que não independe do sistema de coordenadas. Precisamos de uma formulação da condição (P) que independa do sistema de coordenadas em que o campo $L$ é dado. Para isto precisamos da noção de órbitas de um conjunto de campos vetoriais reais suaves, tal definição é dada a seguir.

Seja $\mathcal{M}$ uma variedade paracompacta suave, seja $D$ um conjunto de campos vetoriais reais suaves localmente definidos, isto é, cada $X$ em $D$ está definido em algum subconjunto aberto de $\mathcal{M}$ e é suave neste conjunto. Assuma que a união dos domínios dos elementos de $D$ é igual a $\mathcal{M}$.

Definimos uma relação de equivalência em $\mathcal{M}$ da seguinte forma: dois pontos $p$ e $q$ em $\mathcal{M}$ estão relacionados se existe uma curva $\gamma:[0, T] \longrightarrow \mathcal{M}$ tal que

1) $\gamma(0)=p$ e $\gamma(T)=q$.

2) Existem $t_{0}=0<t_{1}<\cdots<t_{n}=T$ e campos $X_{i} \in D, i=1,2, \ldots, n$ tais que para cada $i$ a restrição $\gamma:\left[t_{i-1}, t_{i}\right] \longrightarrow \mathcal{M}$ é uma curva integral de $X_{i}$ ou $-X_{i}$, isto é $\gamma^{\prime}(t)=X_{i}(\gamma(t))$ ou $\gamma^{\prime}(t)=-X_{i}(\gamma(t))$.

As classes de equivalência desta relação serão chamadas de órbitas de $D$ no sentido se Sussmann.

Definição 4.10. Seja $L=X+i Y$ um campo vetorial complexo e $X, Y$ as partes real e imaginária de L. Definimos as órbitas de Sussmann de L como sendo as órbitas de Sussmann do conjunto $D=\{X, Y\}$.

Observação 4.11. Tendo em mãos esta definição, o Corolário 3.95 implica que se uma órbita de $L$ intersecta o suporte $K$ de uma solução $u$ da equação Lu $=0$, esta deve estar inteiramente contida em K. O qual é equivalente a dizer que $K$ é união de órbitas de $L$. 
Exemplo 4.12. Cosidere o seguinte campo vetorial definido em $\mathbb{R}^{2}$

$$
L=\frac{\partial}{\partial t}+i x \frac{\partial}{\partial x}
$$

Temos que as órbitas deste campo vetorial são: $\mathcal{O}_{1}=\left\{(x, t) \in \mathbb{R}^{2} ; x>0\right\}, \mathcal{O}_{2}=\{(x, t) \in$ $\left.\mathbb{R}^{2} ; x=0\right\}$ e $\mathcal{O}_{3}=\left\{(x, t) \in \mathbb{R}^{2} ; x<0\right\}$.

Exemplo 4.13. Considere agora o campo vetorial definido em $\mathbb{R}^{2}$ por

$$
L=\frac{\partial}{\partial t}+i t \frac{\partial}{\partial x}
$$

Neste caso temos uma única órbita $\mathcal{O}=\mathbb{R}^{2}$.

Observação 4.14. Em [14] Sussmann mostrou que estas órbitas podem ser equipadas com uma topologia e uma estrutura diferenciável natural as quais as tornam subvariedades imersas de $\mathcal{M}$. Além disso, toda órbita é conexa.

Para dar uma formulação da condição $(\mathrm{P})$ independente do sistema de coordenadas escrevemos

$$
L=X+i Y=R e L+i \operatorname{Im} L
$$

Temos que $X \wedge Y \in C^{\infty}\left(\mathbb{R}^{2}, \bigwedge^{2}\left(T\left(\mathbb{R}^{2}\right)\right)\right)$, como $\bigwedge^{2}\left(T\left(\mathbb{R}^{2}\right)\right)$ tem uma seção global que não zera $e_{1} \wedge e_{2}$ e como $\operatorname{dim} \wedge^{2}\left(T\left(\mathbb{R}^{2}\right)\right)=1$, temos que $X \wedge Y$ é múltiplo de $e_{1} \wedge e_{2}$, ou seja, $X \wedge Y=\rho e_{1} \wedge e_{2}, \rho \in C^{\infty}(\Omega, \mathbb{R})$.

Definição 4.15. Dizemos que $X \wedge Y$ não muda de sinal em qualquer órbita de $\{X, Y\}$ quando $X \wedge Y=\rho e_{1} \wedge e_{2}$ temos sempre que $\rho \geq 0$ ou $\rho \leq 0$ nas órbitas de $\{X, Y\}$.

Considere agora um campo de vetores definido em um subconjunto aberto $\Omega \subset \mathbb{R}^{2}$

$$
L=A(x, t) \frac{\partial}{\partial t}+B(x, t) \frac{\partial}{\partial x}
$$

$\operatorname{com}|A(x, t)|+|B(x, t)|>0$, para todo $(x, t) \in \Omega \subset \mathbb{R}^{2}$ e $A, B \in C^{\infty}(\Omega, \mathbb{C})$.

Definição 4.16. Seja $L=X+i Y=R e L+i \operatorname{Im} L$ como em (4.7), dizemos que $L$ satisfaz a condição $(P)$ em $\Omega$ se $X \wedge Y$ não muda de sinal em qualquer órbita de dimensão dois de $L$, isto é, em qualquer órbita de dimensão dois do par de vetores $\{X, Y\}$.

Seja $\varphi \in C^{\infty}\left(\mathbb{R}^{2}\right)$, coloque

$$
Z(x, t)=x+i \varphi(x, t),
$$


e considere o campo vetorial

$$
L=\frac{\partial}{\partial t}-\frac{Z_{t}}{Z_{x}} \frac{\partial}{\partial x}=\frac{\partial}{\partial t}-\frac{i \varphi_{t}(x, t)}{1+i \varphi_{x}(x, t)} \frac{\partial}{\partial x}
$$

Temos que $Z(x, t)$ é uma integral primeira global de $L$, pois $L Z=0$ e $d Z \neq 0$ em $\mathbb{R}^{2}$.

Lema 4.17. Sejam $Z(x, t)$ e L como em (4.8) e (4.9) respectivamente. Se L satisfaz a condição $(P)$ em $\mathbb{R}^{2}$ então a função $\mathbb{R} \ni t \longmapsto \varphi(x, t)$ é monótona para todo $x \in \mathbb{R}$.

Demonstração. Temos que $L=X+i Y$ onde $X=\frac{\partial}{\partial t}+\frac{\varphi_{t} \varphi_{x}}{1+\varphi_{x}^{2}} \frac{\partial}{\partial x}, Y=-\frac{\varphi_{t}}{1+\varphi_{x}^{2}} \frac{\partial}{\partial x}$, então

$$
X \wedge Y=\frac{\varphi_{t}}{1+\varphi_{x}^{2}} \frac{\partial}{\partial x} \wedge \frac{\partial}{\partial t}
$$

Note que $X$ e $Y$ são linearmente dependentes em um ponto se e somente se $\varphi_{t}$ zera neste ponto. Desta maneira as órbitas de dimensão um de $L$ são linhas verticais $x=$ constante nas quais $\varphi_{t}$ é identicamente igual a zero.

Como as órbitas de dimensão dois de $L$ fazem fronteira retas com 0,1 ou 2 órbitas de dimensão um, vemos que cada órbita de dimensão dois $\Omega_{j}, j=1,2$ é da forma $\left(a_{j}, b_{j}\right) \times \mathbb{R}$. Se $L$ satisfaz a condição $(\mathrm{P})$ então $\varphi_{t}$ não muda de sinal em $\Omega_{j}$, digamos que $\varphi_{t} \geq 0 \mathrm{em}$ $\Omega_{j}$, então a função

$$
\mathbb{R} \longmapsto \varphi(x, t)
$$

é monótona crescente para todo $a_{j} \leq x \leq b_{j}$.

Se $x \notin\left(a_{j}, b_{j}\right)$ para qualquer $j$, segue que o ponto de coordenadas $(x, 0)$ pertence a uma órbita de dimensão um, então $\varphi_{t}(x, t)=0$ com $-\infty<t<\infty$ e daí $t \longmapsto \varphi(x, t)$ é constante, isto mostra que $t \longmapsto \varphi(x, t)$ é monótona para todo $x \in \mathbb{R}$.

\subsection{Campos de vetores em várias variáveis}

Agora considere o campo definido em um aberto $\Omega$ de $\mathbb{R}^{n+1}$ dado por

$$
L=\frac{\partial}{\partial t}+\sum_{j=1}^{n} B_{j}(x, t) \frac{\partial}{\partial x_{j}}
$$

onde $B_{j}(x, t)=a_{j}(x, t)+i b_{j}(x, t)$ com $a_{j}, b_{j} \in C^{\infty}(\Omega)$ a valores reais $j=1, \ldots, n$. Como no caso $n=1$, existe um resultado similar ao lema 4.1.

Lema 4.18. Existem coordenadas locais $\xi=x, s=s(x, t)$ definidas em uma vizinhança 
da origem tal que L assume a forma

$$
L=\frac{\partial}{\partial s}+i \sum_{j=1}^{n} \tilde{b}_{j}(\xi, s) \frac{\partial}{\partial \xi_{j}}
$$

com $\tilde{b}_{j}$ suave a valores reais, $j=1, \ldots, n$.

Demonstração. Considere o P.V.I seguinte

$$
\begin{cases}\frac{d x_{j}}{d s}=a_{j}(x, t) & ; x_{j}(0)=\xi_{j}, j=1, \ldots, n \\ \frac{d t}{d s}=1 & ; t(0)=0\end{cases}
$$

com solução $(x(\xi, s), t(\xi, s))$ dada por

$$
\begin{aligned}
x_{j}(\xi, s) & =\xi_{j}+\int_{0}^{s} a_{j}(x(\xi, \sigma), \sigma) d \sigma, j=1, \ldots, n \\
t(\xi, s) & =s .
\end{aligned}
$$

Temos que $x_{j}(\xi, 0)=\xi_{j}, j=1, \ldots, n, \log \mathrm{o}$

$$
\frac{\partial x_{j}}{\partial \xi_{j}}(0,0)=1, \quad j=1, \ldots, n \quad \text { e } \quad \frac{\partial t}{\partial s}=1,
$$

então o determinante jacobiano fica

$$
\left|\begin{array}{cccc}
\frac{\partial x_{1}}{\partial \xi_{1}}(0,0) & \cdots & \frac{\partial x_{1}}{\partial \xi_{n}}(0,0) & \frac{\partial x_{1}}{\partial s}(0,0) \\
\vdots & \ddots & \vdots & \vdots \\
\frac{\partial x_{n}}{\partial \xi_{1}}(0,0) & \cdots & \frac{\partial x_{n}}{\partial \xi_{n}}(0,0) & \frac{\partial x_{n}}{\partial s}(0,0) \\
\frac{\partial t}{\partial \xi_{1}}(0,0) & \cdots & \frac{\partial t}{\partial \xi_{n}}(0,0) & \frac{\partial t}{\partial s}(0,0)
\end{array}\right|=\left|\begin{array}{cccc}
1 & \cdots & 0 & \frac{\partial x_{1}}{\partial s}(0,0) \\
\vdots & \ddots & \vdots & \vdots \\
0 & \cdots & 1 & \frac{\partial x_{n}}{\partial s}(0,0) \\
0 & \cdots & 0 & 1
\end{array}\right|=1 \neq 0
$$

o que garante que a aplicação $(\xi, s) \longrightarrow(x(\xi, s), t(\xi, s))$ é localmente uma mudança de variável. Ainda pela regra da cadeia temos

$$
\begin{aligned}
& \frac{\partial}{\partial s}=\frac{\partial t}{\partial s} \frac{\partial}{\partial t}+\frac{\partial x_{1}}{\partial s} \frac{\partial}{\partial x_{1}}+\cdots+\frac{\partial x_{n}}{\partial s} \frac{\partial}{\partial x_{n}}=\frac{\partial}{\partial t}+a_{1}(x, t) \frac{\partial}{\partial x_{1}}+\cdots+a_{n}(x, t) \frac{\partial}{\partial x_{n}} \\
& \frac{\partial}{\partial \xi_{j}}=\frac{\partial t}{\partial \xi_{j}} \frac{\partial}{\partial t}+\frac{\partial x_{j}}{\partial \xi_{j}} \frac{\partial}{\partial x_{1}}+\cdots+\frac{\partial x_{n}}{\partial \xi_{j}} \frac{\partial}{\partial x_{n}}=\frac{\partial x_{1}}{\partial \xi_{j}} \frac{\partial}{\partial x_{1}}+\cdots+\frac{\partial x_{n}}{\partial \xi_{j}} \frac{\partial}{\partial x_{n}}
\end{aligned}
$$


Como em uma vizinhança da origem temos que

$$
\left|\begin{array}{ccc}
\frac{\partial x_{1}}{\partial \xi_{1}} & \cdots & \frac{\partial x_{1}}{\partial \xi_{n}} \\
\vdots & \ddots & \vdots \\
\frac{\partial x_{n}}{\partial \xi_{1}} & \cdots & \frac{\partial x_{n}}{\partial \xi_{n}}
\end{array}\right| \neq 0
$$

podemos isolar $\frac{\partial}{\partial x_{j}}$ em (4.12) e obter

$$
\frac{\partial}{\partial x_{j}}=c_{j}(x, t) \frac{\partial}{\partial \xi_{j}}, \quad j=1, \ldots, n .
$$

Portanto, nestas coordenadas obtemos $L$ na forma desejada

$$
\begin{aligned}
L & =\frac{\partial}{\partial t}+\sum_{j=1}^{n}\left(a_{j}(x, t)+b_{j}(x, t)\right) \frac{\partial}{\partial x_{j}}=\frac{\partial}{\partial t}+\sum_{j=1}^{n} a_{j}(x, t) \frac{\partial}{\partial x_{j}}+i \sum_{j=1}^{n} b_{j}(x, t) \frac{\partial}{\partial x_{j}} \\
& =\frac{\partial}{\partial s}+i \sum_{j=1}^{n} b_{j} c_{j} \frac{\partial}{\partial \xi_{j}}=\frac{\partial}{\partial s}+i \sum_{j=1}^{n} \tilde{b}_{j} \frac{\partial}{\partial \xi_{j}} .
\end{aligned}
$$

Como antes, escrevemos $L=X+i Y \operatorname{com} X=R e L$ e $Y=\operatorname{Im} L$ e nos referimos às órbitas do par de vetores reais $\{X, Y\}$ como as órbitas de $L$. Notemos que $X$ e $Y$ não zeram simultaneamente e daí $L$ não tem órbitas de dimensão zero.

Seja $\Sigma$ uma órbita de $L$ de dimensão dois e assuma que $\Sigma$ é orientável. Existe uma seção global que não se anula $\rho \in C^{\infty}\left(\Sigma, \bigwedge^{2}(T(\Sigma))\right)$. Ambos $X$ e $Y$ são tangente a $\Sigma$. Assim, eles podem ser considerados como seções do fibrado tangente $T(\Sigma) \longrightarrow \Sigma$ que produz uma seção $X \wedge Y$ do fibrado $\wedge^{2}(T(\Sigma)) \longrightarrow \Sigma$. Logo, $X \wedge Y=b \rho$ onde $b$ é uma função real suave definida em $\Sigma$.

Definição 4.19. Se b não assume sinal oposto em $\Sigma$ dizemos que $X \wedge Y$ não muda de sinal em $\Sigma$.

Notemos que se $\rho_{1}$ é outra seção global que não zera em $\Sigma$ de $\bigwedge^{2}(T(\Sigma)) \longrightarrow \Sigma$, então $\rho_{1}=\lambda \rho \operatorname{com} \lambda$ função real suave e $\lambda \neq 0$ em $\Sigma$. Como $\Sigma$ é conexo então $\lambda>0$ ou $\lambda<0$. Isto mostra que a noção $X \wedge Y$ não muda de sinal em $\Sigma$ é independente do gerador $\rho$.

Definição 4.20. Dizemos que o campo $L=X+i Y$ dado acima satisfaz a condição $(P)$ em $\Omega$ se e somente se 
1) As órbitas de $L$ em $\Omega$ tem dimensão no máximo dois.

2) As órbitas de dimensão dois de $L$ são orientáveis e $X \wedge Y$ não muda de sinal em qualquer órbita de dimensão dois de $L$.

Proposição 4.21. Seja L como em (4.10) satisfazendo a condição (P) em $\Omega$ e $h \in$ $C^{\infty}(\Omega, \mathbb{C})$ uma função que não se anula. Então $L^{\prime}=h L$ satisfaz a condição $(P)$ em $\Omega$.

Demonstração. Escreva $h=\alpha+i \beta \operatorname{com} \alpha, \beta \in C^{\infty}(\Omega, \mathbb{R})$, então $L^{\prime}=X^{\prime}+i Y^{\prime}$ com $X^{\prime}=\alpha X-\beta Y$ e $Y^{\prime}=\alpha Y+\beta X$.

As órbitas de $L$ e $L^{\prime}$ são idênticas porque ambos geram o mesmo fibrado. Então $L^{\prime}$ não tem órbitas de dimensão maior que dois. Logo, se $\Sigma$ é uma órbita de $L^{\prime}$ de dimensão dois, como $\Sigma$ também é uma órbita de $L$, temos que $X \wedge Y$ não muda de sinal sobre $\Sigma$ e portanto $X^{\prime} \wedge Y^{\prime}=\left(\alpha^{2}+\beta^{2}\right) X \wedge Y$ não muda de sinal sobre $\Sigma$, o qual implica que $L^{\prime}$ satisfaz a condição $(\mathrm{P})$.

Se $L$ é dado por (4.11), definido em $\Omega=\left\{x \in \mathbb{R}^{n} ;|x|<r\right\} \times(-T, T)$, vamos descrever as órbitas de dimensão um e dois de $L$.

Como $X=\frac{\partial}{\partial t}$, as órbitas de $X$ em $\Omega$ são os segmentos verticais $\left\{x_{0}\right\} \times(-T, T)$. Desta maneira, se $\left(x_{0}, t_{0}\right)$ pertence a uma órbita $\Sigma$ de $L$, segue que $\left\{x_{0}\right\} \times(-T, T) \subset \Sigma$ e isto implica que toda órbita de $L$ de dimensão qualquer pode ser escrita como uma união de segmentos verticais.

Agora se $\Sigma$ é uma órbita de dimensão um de $L$, então $X$ e $Y$ são linearmente dependentes em todos os pontos de $\Sigma$ e, assim, $Y=\sum_{j=1}^{n} b_{j} \frac{\partial}{\partial x_{j}}$ deve ser identicamente zero em $\Sigma$, o que implica que $\Sigma=\left\{x_{0}\right\} \times(-T, T)$ para algum $x_{0} \in\left\{x \in \mathbb{R}^{n} ;|x|<r\right\}$ tal que $b_{j}\left(x_{0}, t\right)=0$ para $j=1, \ldots, n$ e $|t|<T$.

Se $\Sigma$ é uma órbita de dimensão maior ou igual a dois de $L$ com $\left(x_{0}, t_{0}\right) \in \Sigma$, então existe $\left(x_{0}, t_{1}\right) \in \Sigma$ tal que $Y\left(x_{0}, t_{1}\right) \neq 0$ pois, caso contrário, $\Sigma=\left\{x_{0}\right\} \times(-T, T)$ o que não é possível pois a dimensão de $\Sigma$ é maior ou igual a dois.

Considere a curva integral maximal $\gamma$ em $\left\{x \in \mathbb{R}^{n} ;|x|<r\right\}$ passando pelo ponto $x_{0}$ do campo $Y\left(x, t_{1}\right), x \in\left\{x \in \mathbb{R}^{n} ;|x|<r\right\}$. Então $\gamma \times(-T, T)$ é um subconjunto fechado de $\Sigma$ e também uma variedade de dimensão dois. Desta maneira se a dimensão de $\Sigma$ é dois segue por conexidade que $\Sigma=\gamma \times(-T, T)$ e, em particular, toda órbita de dimensão dois de $L$ é orientavel.

Observe que $Y\left(., t_{1}\right)$ não é identicamente zero em $\gamma$ (caso contrario $\gamma$ reduziria a um único ponto). Se $v(x)=Y\left(x, t_{1}\right)$, então $\rho=v \wedge \frac{\partial}{\partial t} \in \bigwedge^{2}(\Sigma)$ nunca zera.

Proposição 4.22. Seja $L$ dado por (4.11) em $\Omega=\left\{x \in \mathbb{R}^{n} ;|x|<r\right\} \times(-T, T)$. Se $L$ satisfaz a condição (P) em $\Omega$, então para todo $x \in\left\{x \in \mathbb{R}^{n} ;|x|<r\right\}$ e $\xi \in \mathbb{R}^{n}$ a função $(-T, T) \ni t \longmapsto \sum_{j=1}^{n} b_{j}(x, t) \xi_{j}$ não muda de sinal. 
Demonstração. Seja $Y(x, t)=\left(b_{1}(x, t), \ldots, b_{n}(x, t)\right)$, assuma que $L$ satisfaz a condição (P) e sejam $x_{0} \in\left\{x \in \mathbb{R}^{n} ;|x|<r\right\}$ e $\xi_{0} \in \mathbb{R}^{n}$.

Se $\left(x_{0}, t_{0}\right)$ pertence a uma órbita de dimensão um de $L$ para algum $t_{0} \in(-T, T)$ então temos que $Y\left(x_{0}, t\right)=0$ em $|t|<T$ e obviamente $(-T, T) \ni t \longmapsto Y\left(x_{0}, t\right) \xi_{0}=0$ e não muda de sinal. Daí podemos supor que $Y\left(x_{0}, t_{0}\right) \neq 0$ para algum $t_{0} \in(-T, T)$. Assim $\left(x_{0}, t_{0}\right) \in \Sigma$ onde $\Sigma$ é uma órbita de dimensão dois no qual $X \wedge Y$ não muda de sinal, pois $L$ satisfaz a codição $(\mathrm{P})$.

Seja agora $\gamma$ a curva integral de $v(x)=Y\left(x, t_{0}\right)$ em $\left\{x \in \mathbb{R}^{n} ;|x|<r\right\}$ passando por $x_{0}$, temos que $\Sigma=\gamma \times(-T, T)$ e $\rho=\frac{\partial}{\partial t} \wedge v$ gera $\bigwedge^{2}(\Sigma)$ em todo ponto de $\Sigma$.

Seja $\left(x_{0}, t\right) \in \Sigma$. Como $Y$ é um vetor horizontal tangente a $\gamma \times(-T, T)$ vemos que $Y\left(x_{0}, t\right)=\lambda\left(x_{0}, t\right) v\left(x_{0}\right), t \in(-T, T)$. Além disso,

$$
\begin{aligned}
X \wedge Y\left(x_{0}, t\right) & =\frac{\partial}{\partial t} \wedge\left(\lambda\left(x_{0}, t\right) v\left(x_{0}\right)\right) \\
& =\lambda\left(x_{0}, t\right) \frac{\partial}{\partial t} \wedge v\left(x_{0}\right) \\
& =\lambda\left(x_{0}, t\right) \rho\left(x_{0}, t\right)
\end{aligned}
$$

e como $X \wedge Y$ não muda de sinal, temos que $\lambda\left(x_{0}, t\right) \geq 0$ ou $\lambda\left(x_{0}, t\right) \leq 0$ em $(-T, T)$. Isto mostra que o vetor $(-T, T) \ni t \longmapsto Y\left(x_{0}, t\right)$, não muda de direção e sentido e, portanto, $(-T, T) \ni t \longmapsto Y\left(x_{0}, t\right) \xi_{0}$ não muda de sinal.

Observação 4.23. Se $L$ dado por (4.11) em $\Omega=B(0, r) \times(-T, T)$ satisfaz a condição (P) em $\Omega$, vem da Proposição 4.22 que existe $v(x)$ vetor suave tal que

$$
\vec{b}(x, t)=\left(b_{1}(x, t), \ldots, b_{n}(x, t)\right)=Y(x, t)=\lambda(x, t) v(x)
$$

e para cada $x \in B(0, r)$,

$$
\lambda(x, .) \geq 0 \quad \text { ou } \quad \lambda(x, .) \leq 0 \quad \text { em } \quad(-T, T)
$$

$\log o$

$$
\begin{aligned}
\vec{b}(x, t) & =\lambda(x, t) v(x) \\
& =\lambda(x, t) \frac{v(x)}{|v(x)|}|v(x)| \\
& =\operatorname{sgn}[\lambda(x, t)] \lambda(x, t) \frac{\operatorname{sgn}[\lambda(x, t)] v(x)}{|v(x)|}|v(x)|
\end{aligned}
$$




$$
\begin{aligned}
& =|\lambda(x, t)||v(x)| v(x) \\
& =|\vec{b}(x, t)| v(x) .
\end{aligned}
$$

Resumindo temos que se $L$ satisfaz a condição $(P)$, existe um vetor suave unitário $v(x)$ definido sobre $\mathbb{R}^{n}$ tal que

$$
\vec{b}(x, t)=|\vec{b}(x, t)| v(x), \quad x \in B(0, r), t \in(-T, T) .
$$

Note que $v\left(x_{0}\right)$ pode ser definido arbitrariamente se $\vec{b}(x, t)=0$ para todo $t$.

Sejam agora

$$
\mathcal{N}=\left\{x \in \mathbb{R}^{n} ; \vec{b}(x, t)=0,|t|<1\right\}
$$

e

$$
\rho(x)=\sup _{|t| \leq 1}|\vec{b}(x, t)|, x \in \mathbb{R}^{n} .
$$

Observe que $\mathcal{N}$ é precisamente o conjunto onde $\rho$ zera.

Lema 4.24. Sejam $\rho$ e $\mathcal{N}$ definidos como acima. Então a função $\rho$ é Lipschitziana e

$$
\|\nabla \rho\|_{L^{\infty}} \leq\left\|\nabla_{x} \vec{b}\right\|_{L^{\infty}}
$$

Demonstração. Sejam $x, y \in \mathbb{R}^{n}$, considere $t \in[-1,1]$ tal que $\rho(x)=|\vec{b}(x, t)|$. Logo

$$
\begin{aligned}
\rho(x) & =|\vec{b}(x, t)| \\
& \leq|\vec{b}(x, t)-\vec{b}(y, t)|+|\vec{b}(y, t)| \\
& \leq\left|\nabla_{x} \vec{b}(x, t)\right||x-y|+\rho(y)
\end{aligned}
$$

daí vem que

$$
\rho(x)-\rho(y) \leq\left\|\nabla_{x} \vec{b}(x, t)\right\|_{L^{\infty}}|x-y| .
$$

Trocando os papéis de $x$ e $y$ obtemos também que

$$
\rho(y)-\rho(x) \leq\left\|\nabla_{x} \vec{b}(x, t)\right\|_{L^{\infty}}|x-y|
$$

Portanto obtemos que $\rho$ é Lipschitziana, ou seja,

$$
|\rho(x)-\rho(y)| \leq\left\|\nabla_{x} \vec{b}\right\|_{L^{\infty}}|x-y|, \quad \forall x, y \in \mathbb{R}^{n}
$$

Mais ainda, isto implica que

$$
\|\nabla \rho\|_{L^{\infty}} \leq\left\|\nabla_{x} \vec{b}\right\|_{L^{\infty}}
$$


Observação 4.25. Sabemos que

$$
\vec{b}(x, t)=|\vec{b}(x, t)| v(x), \quad x \in \mathbb{R}^{n} .
$$

Como $v(x)$ é vetor unitário, temos que para cada $x$ existe $j=j(x)$ tal que $v_{j}(x) \neq 0$, podemos assumir sem perda de generalidade que $v_{1}(x) \neq 0$.

Considere o sistema

$$
\left\{\begin{aligned}
\frac{d x_{j}}{d y_{1}} & =\frac{v_{j}(x)}{v_{1}(x)}, \quad j=1, \ldots, n . \\
x_{j}(0) & =y_{j}
\end{aligned}\right.
$$

Logo $x_{1}=y_{1}$ e $x_{j}=x_{j}\left(y_{1}, \ldots, y_{n}\right)$ é uma mudança de variável em torno da origem, portanto

$$
\begin{aligned}
\frac{\partial}{\partial y_{1}} & =\frac{\partial}{\partial x_{1}} \frac{d x_{1}}{d y_{1}}+\frac{\partial}{\partial x_{2}} \frac{d x_{2}}{d y_{1}}+\cdots+\frac{\partial}{\partial x_{n}} \frac{d x_{n}}{d y_{1}} \\
& =\frac{\partial}{\partial x_{1}}+\frac{v_{2}}{v_{1}} \frac{\partial}{\partial x_{2}}+\cdots+\frac{v_{n}}{v_{1}} \frac{\partial}{\partial x_{n}} .
\end{aligned}
$$

Obtemos que

$$
\begin{aligned}
L & =\frac{\partial}{\partial t}+i \sum_{j=1}^{n} b_{j}(x, t) \frac{\partial}{\partial x_{j}} \\
& =\frac{\partial}{\partial t}+i|\vec{b}(x, t)| \sum_{j=1}^{n} v_{j}(x) \frac{\partial}{\partial x_{j}} \\
& =\frac{\partial}{\partial t}+i|\vec{b}(x, t)| v_{1}(x) \sum_{j=1}^{n} \frac{v_{j}(x)}{v_{1}(x)} \frac{\partial}{\partial x_{j}} \\
& =\frac{\partial}{\partial t}+i|\vec{b}(x(y), t)| v_{1}(x) \frac{\partial}{\partial y_{1}},
\end{aligned}
$$

daí segue que sobre as órbitas de dimensão dois o campo L fica da seguinte forma

$$
L=\frac{\partial}{\partial t}+i \vec{b}_{1}(x(y), t) \frac{\partial}{\partial y_{1}}
$$

Onde $\vec{b}_{1}(x(y), t)=|\vec{b}(x(y), t)| v_{1}(x)$.

Teorema 4.26. Se L é localmente resolúvel em $C^{\infty}$ então L é localmente integrável.

Demonstração. Considere $U_{j}$ a solução no sentido de série de potência formal do problema 
de Cauchy não característico seguinte

$$
\left\{\begin{array}{cl}
L U_{j} & =0 \\
U_{j}(x, 0) & =x_{j}, j=1, \ldots, m .
\end{array}\right.
$$

Os coeficientes da série formal $U_{j}$ correspondentes ao monômios que não contêm $t$ são determinados pela condição inicial $U_{j}(x, 0)$, isto é, eles são todos zero com exceção do coeficiente de $x_{j}$ que é igual a 1 . Os coeficientes dos monômios da forma $t^{l} x^{\alpha}$ são determinados de $L U_{j}=0$ indutivamente em $l$.

Uma vez que as séries formais sejam obtidas, usando o Teorema de Borel, tomamos $Z_{j}^{\sharp}$ uma função suave que tem $U_{j}$ como sua série de Taylor na origem. Por sua própria definição temos que $Z_{1}^{\sharp}, \ldots, Z_{m}^{\sharp}$ satisfazem $L Z_{j}^{\sharp}=O\left(|(x, t)|^{k}\right), k=0,1, \ldots$ e $d Z_{1}^{\sharp}(0), \ldots, d Z_{m}^{\sharp}(0)$ são linearmente independentes.

Para obter as integrais primeiras exatas por correção de $Z_{1}^{\sharp}, \ldots, Z_{m}^{\sharp}$, devemos resolver as equações $L u_{j}=L Z_{j}^{\sharp}=f_{j}, j=1, \ldots, m$ numa vizinhança da origem e definimos $Z_{j}=Z_{j}^{\sharp}-u_{j}$. Claramente $L Z_{j}=0$. Basta mostrar agora que $d Z_{1}(0), \ldots, d Z_{m}(0)$ são linearmente independentes. Isto será garantido se nós tomarmos $\left|d u_{j}(0)\right|$ pequeno.

Seja $K$ uma bola centrada na origem tal que $L C^{\infty}(K)=C^{\infty}(K)$ e seja $\mathcal{H}$ o subespaço de $C^{\infty}(K)$ tal que $L h=0, h \in C^{\infty}(K)$. Então $L$ define uma função linear contínua e sobrejetora

$$
L: \frac{C^{\infty}(K)}{\mathcal{H}} \longrightarrow C^{\infty}(K)
$$

definida por $L \bar{f}=L f$, a qual pelo Teorema da aplicação aberta (para espaços de Fréchet) tem uma inversa contínua

$$
L^{-1}: C^{\infty}(K) \longrightarrow \frac{C^{\infty}(K)}{\mathcal{H}}
$$

Isto significa, em particular, que dado $\epsilon>0$ existem $\delta>0$ e $k \in \mathbb{Z}^{+}$tais que para toda $f \in C^{\infty}(K)$ tal que $\left\|D^{\beta} f\right\|_{L^{\infty}(K)}<\delta$, para todo $|\beta| \leq k$ existe $u \in C^{\infty}(K)$ tal que $L u=f \mathrm{e}\|d u\|_{L^{\infty}(K)}<\epsilon$.

Seja $\chi(x, t) \in C_{c}^{\infty}\left(\mathbb{R}^{n+1}\right)$ tal que $\chi(x, t)=1$ em $B(0,1)$ e considere $f_{j, \rho}(x, t)=f_{j}(x, t) \chi(\rho x, \rho t)$ (observe que $|\rho \cdot(x, t)|<1 \Leftrightarrow|(x, t)|<1 / \rho)$.

Como $f_{j}$ zera de ordem infinita na origem vemos que, escolhendo $\rho$ suficientemente grande, $\left\|D^{\beta} f_{j, \rho}\right\|_{L^{\infty}}<\delta$ para todo $|\beta| \leq k$. Escolha agora $u_{j}$ tal que $L u_{j}=f_{j, \rho}$ e $\left\|d u_{j}\right\|_{L^{\infty}(K)}<\epsilon$. Como $f_{j, \rho}=f_{j}=L Z_{j}^{\sharp}$ em $B(0,1 / \rho)$ vemos que as funções $Z_{j}=Z_{j}^{\sharp}-u_{j}, j=1, \ldots, m$ formam um conjunto completo de integrais primeiras em uma vizinhança da origem se $\epsilon$ é tomado suficientemente pequeno. 


\section{- \\ Teorema de Radó para campos vetoriais localmente resolúveis}

Neste capítulo apresentamos de forma detalhada o resultado obtido por J.Hounie e J. Tavares em [7], isto é, vamos provar que todo campo vetorial com coeficientes suaves definidos em um subconjunto aberto $\Omega \subset \mathbb{R}^{n+1}$ que é localmente resolúvel, tem a propriedade de Radó. A prova deste resultado explora duas propriedades desta classe de campos vetoriais: o seu caráter essencialmente bidimensional (expresso pelo fato de que as órbitas têm dimensão no máximo dois) e a propriedade da integrabilidade local que permite a redução do problema à versão clássica do Teorema de Radó no plano. Uma outra observação a fazer é que neste capítulo assumimos que a condição (P) é condição necessária e suficiente para a resolubilidade local. Para a demonstração deste fato pode-se ver [1].

Iniciaremos nossa discussão deste capítulo com a seguinte definição.

Definição 5.1. Seja $L$ um campo vetorial definido em um aberto $\Omega$ de $\mathbb{R}^{N}$. Dizemos que $u \in C^{0}(\Omega)$ é uma função de Radó para $L$ se

$$
L u=0 \quad \text { em } \quad \Omega \backslash u^{-1}(0)
$$

no sentido das distribuições. Se toda função de Radó para L é uma solução fraca de $L u=0$ sobre $\Omega$ (i.e. $\langle L u, \varphi\rangle=0, \forall \varphi \in C_{c}^{\infty}(\Omega)$ ), dizemos que L tem a propriedade Radó. Ou seja o conjunto singular onde u zera e a equação é a priori não satisfeita pode ser removido e a equação $L u=0$ se satisfaz em todo $\Omega$.

Daqui em diante vamos considerar $L$ um campo vetorial definido em um subconjunto 
aberto $\Omega \subset \mathbb{R}^{n+1}$.

Lema 5.2. Seja L localmente integrável e u uma função de Radó para L. Então u é constante sobre as órbitas de $L$ de dimensão um.

Demonstração. Seja $\Sigma$ uma órbita de $L$ de dimensão um. Se $u$ é identicamente zero sobre $\Sigma$ então não temos nada a provar.

Considere um ponto $p \in \Sigma$ tal que $u(p) \neq 0$. Como $L$ é localmente integrável, pelo Corolário 3.79 podemos escolher coordenadas locais $(x, t)$ numa vizinhança de $p$ tal que $x(p)=t(p)=0$ e funções suaves

$$
Z_{j}(x, t)=x_{j}+i \phi_{j}(x, t), j=1, \ldots, n ;|x|<1,|t|<1
$$

$\operatorname{com} \phi_{j}(0,0)=d_{x} \phi_{j}(0,0)=0$ e $L Z_{j}=0$.

Logo, pela Observação 3.81 podemos considerar que $L$ é dado da seguinte forma

$$
L=\frac{\partial}{\partial t}+\sum_{j=1}^{n} \lambda_{j}(x, t) \frac{\partial}{\partial x_{j}}
$$

onde os coeficientes $\lambda_{j}$ são determinados pelas equações $L Z_{j}=0, j=1, \ldots, n$. Além disso, tendo presente o comentário feito antes da Proposição 4.22, vem que em nosso sistema de coordenadas locais a órbita $\Sigma$ é dada por

$$
\Sigma=\{0\} \times(-1,1)=\{(0, s) ;-1<s<1\} .
$$

Observe também que a curva integral de $X=\partial / \partial t$ que passa pela origem é um segmento vertical contido em $\Sigma$. Logo as equações $L Z_{j}=0$ restritas a $\Sigma$ significam que para cada $j$

$$
\frac{\partial}{\partial s} Z_{j}(0, s)=0 \Rightarrow \frac{\partial}{\partial s} \phi_{j}(0, s)=0, \forall s \in(-1,1) \Rightarrow \phi_{j}(0, s)=C t e .
$$

Mais ainda, essa constante é zero, pois $\phi_{j}(0,0)=0$ para todo $j=1, \ldots, n$.

Escrevendo $Z=\left(Z_{1}, \ldots, Z_{n}\right)$ e $\phi=\left(\phi_{1}, \ldots, \phi_{n}\right)$, vem que se $q \in \Sigma$, isto é $q=(0, s)$ com $-1<s<1$, então

$$
Z(q)=Z(0, s)=i \phi(0, s)=0=Z(0,0)
$$

ou seja, $q$ pertence a uma fibra de $Z$, o qual implica que $\Sigma$ está contida em uma fibra de $Z$.

Por outro lado como $u(0,0) \neq 0$ e $u$ é contínua então existe $\epsilon>0$ tal que $u(x, t) \neq 0$ 
para $|x|<\epsilon,|t|<\epsilon$. Como $u$ é uma função de Radó para $L$ vem que

$$
L u=0, \text { para }|x|<\epsilon,|t|<\epsilon
$$

Usando o Corolário 3.89, corolário do teorema de aproximação, temos que $u$ é constante sobre as fibras de $Z$. Em particular $u$ é constante sobre $\Sigma$ para $|s|$ pequeno, mas como $\Sigma$ é conexo segue que $u$ é constante sobre $\Sigma$.

Vamos fazer uma observação importante que usaremos depois na demonstração do Lema 5.6.

Observação 5.3. Se $P\left(x, y, D_{x}\right)$ é um operador diferencial com coeficientes dependendo das variáveis $x \in \mathbb{R}^{n}$ e $y \in \mathbb{R}^{m}$, contendo derivadas apenas com relação a $x$. Se $u(x, y)$ é uma solução fraca contínua de $P\left(x, y, D_{x}\right) u=0 \mathrm{em} \mathbb{R}^{n+m}$ (i.e. $\langle P u, \psi\rangle=0$ para todo $\psi \in C_{c}^{\infty}\left(\mathbb{R}^{n+m}\right)$ ), então $v(x)=u\left(x, y_{0}\right)$ é uma solução fraca de $P\left(x, y_{0}, D_{x}\right) v=0$ em $\mathbb{R}^{n}$.

De fato, considere a função $\psi(x, y)=\varphi(x) \phi(y)$, com $\varphi \in C_{c}^{\infty}\left(\mathbb{R}^{n}\right)$ e $\phi \in C_{c}^{\infty}\left(\mathbb{R}^{m}\right)$. Assim, $\psi \in C_{c}^{\infty}\left(\mathbb{R}^{n+m}\right) e$

$$
\left\langle P\left(x, y, D_{x}\right) u(x, y), \varphi(x) \phi(y)\right\rangle=0
$$

ou seja,

$$
\begin{array}{r}
\iint_{\mathbb{R}^{n+m}} u(x, y) P^{t}\left(x, y, D_{x}\right)[\varphi(x) \phi(y)] d x d y=0 \\
\int_{\mathbb{R}^{m}} \int_{\mathbb{R}^{n}} u(x, y) \phi(y) P^{t}\left(x, y, D_{x}\right)[\varphi(x)] d x d y=0 \\
\int_{\mathbb{R}^{m}}\left\{\int_{\mathbb{R}^{n}} u(x, y) P^{t}\left(x, y, D_{x}\right)[\varphi(x)] d x\right\} \phi(y) d y=0 .
\end{array}
$$

Se escrevemos

$$
H(y)=\int_{\mathbb{R}^{n}} u(x, y) P^{t}\left(x, y, D_{x}\right)[\varphi(x)] d x
$$

e substituimos em (5.2) temos que

$$
\int_{\mathbb{R}^{m}} H(y) \phi(y) d y=0, \text { para toda } \phi \in C_{c}^{\infty}\left(\mathbb{R}^{m}\right) .
$$

Como $H$ é contínua usando a Observação 1.17 temos que $H(y)=0$ para todo $y \in \mathbb{R}^{m}$; em particular, $H\left(y_{0}\right)=0$, ou seja,

$$
\int_{\mathbb{R}^{n}} v(x) P^{t}\left(x, y_{0}, D_{x}\right)[\varphi(x)] d x=\int_{\mathbb{R}^{n}} u\left(x, y_{0}\right) P^{t}\left(x, y_{0}, D_{x}\right)[\varphi(x)] d x=0 \quad \text { para toda } \varphi \in C_{c}^{\infty}\left(\mathbb{R}^{n}\right) .
$$


Portanto $v(x)=u\left(x, y_{0}\right)$ é uma solução fraca de $P\left(x, y_{0}, D_{x}\right) v=0 \mathrm{em} \mathbb{R}^{n}$.

\section{$5.1 \quad \mathrm{O}$ resultado principal}

Nesta seção vamos mostrar o resultado principal deste trabalho que enunciamos a seguir

Teorema 5.4. Seja L um campo vetorial definido em um subconjunto aberto $\Omega \subset \mathbb{R}^{n+1}$ satifazendo a condição (P). Então L tem a propriedade Radó.

Antes de provar o teorema vamos fazer algumas observações e lembrar alguns resultados do capítulo anterior. Introduzindo coordenadas $(x, t)$ vamos escrever

$$
L=A(x, t) \frac{\partial}{\partial t}+\sum_{j=1}^{n} B_{j}(x, t) \frac{\partial}{\partial x_{j}}
$$

com coeficiente complexos $A, B_{1}, \ldots, B_{n} \in C^{\infty}(\Omega)$ tal que

$$
|A(x, t)|+\sum_{j=1}^{n}\left|B_{j}(x, t)\right|>0, \text { para todo }(x, t) \in \Omega .
$$

Usando o Lema 4.18 podemos considerar o campo vetorial $L$ da seguinte forma

$$
L=\frac{\partial}{\partial t}-i \sum_{j=1}^{n} b_{j}(x, t) \frac{\partial}{\partial x_{j}}, \quad|x| \leq 1,|t| \leq 1
$$

onde os coeficientes $b_{j}(x, t)$ são funções suaves a valores reais . Vamos denotar por $\vec{b}(x, t)$ o campo vetorial em $\mathbb{R}^{n}$ dado por

$$
\vec{b}(x, t)=\sum_{j=1}^{n} b_{j}(x, t) \frac{\partial}{\partial x_{j}}, \quad|x| \leq 1,|t| \leq 1 .
$$

Na Observação 4.23 vimos que se $L$ satisfaz a condição $(\mathrm{P})$, então existe um vetor unitário $\vec{v}(x)$ definido no $\mathbb{R}^{n}$ tal que

$$
\vec{b}(x, t)=|\vec{b}(x, t)| \vec{v}(x), \quad x \in \mathbb{R}^{n}, t \in \mathbb{R},|x| \leq 1,|t| \leq 1 .
$$

Seja agora

$$
\mathcal{N}=\left\{x \in \mathbb{R}^{n} ; \vec{b}(x, t)=0,|x|<1,|t|<1\right\}
$$


e

$$
\rho(x)=\sup _{|t| \leq 1}|\vec{b}(x, t)|, \quad x \in \mathbb{R}^{n},|x|<1,
$$

de modo que $\mathcal{N}$ é precisamente o conjunto onde $\rho$ zera. Mostramos no Lema 4.24 que $\rho$ é uma função Lipschitziana e $\|\nabla \rho\|_{L^{\infty}} \leq\left\|\nabla_{x} \vec{b}\right\|_{L^{\infty}}$. Assumindo, sem perda de generalidade, que $\left\|\nabla_{x} \vec{b}\right\|_{L^{\infty}} \leq 1$, obtemos a seguinte estimativa

$$
\left|\rho(x)-\rho\left(x^{\prime}\right)\right| \leq\left|x-x^{\prime}\right|, \quad x, x^{\prime} \in \mathbb{R}^{n},|x|,\left|x^{\prime}\right|<1
$$

Para mostrar o Teorema 5.4, ou seja, para mostrar que $L u=0$ no sentido das distribuições em $\Omega$, para toda função de Radó, vamos fixar um ponto $p \in \Omega$ que tomaremos como sendo a origem e mostrar que $L u=0$ no sentido das distribuições em uma vizinhança da origem, com $L$ dado por (5.3). Mais precisamente, vamos fixar $Q$, o cubo unitário em $\mathbb{R}^{n+1}$ e mostrar que $L u=0$ no sentido das distribuições em $Q$, ou equivalentemente

$$
\iint u(x, t) L^{t} \phi d x d t=\iint u(x, t) \chi(x) L^{t} \phi d x d t+\iint u(x, t)(1-\chi(x)) L^{t} \phi d x d t=0
$$

para qualquer $\phi \in C_{c}^{\infty}(Q)$, onde $\chi$ é a função característica do conjunto $\mathcal{N}$.

Vamos mostrar então que cada parcela da soma acima é zero.

Lema 5.5. Sejam $\chi$ a função característica de $\mathcal{N}$ e $\phi \in C_{c}^{\infty}\left(\mathbb{R}^{n+1}\right)$ suportada no cubo unitário $Q$. Assuma que a função contínua u satisfaz $L u=0$ onde $u \neq 0$, então

$$
\iint u(x, t) \chi(x) L^{t} \phi d x d t=0
$$

Demonstração. Se $x \in \mathcal{N}$, então $b(x, t)=0$ para $|t|<1$ logo o segmento $\{x\} \times(-1,1)$ está contido em uma órbita de $L$ de dimensão um. Pelo Lema 5.2 temos que a função $u$ restrita a este segmento é constante em $t$, ou seja

$$
\{x\} \times(-1,1) \ni(x, t) \longmapsto u(x, t)=k_{x}
$$

Logo a função $u$ restrita ao conjunto $\mathcal{N} \times(-1,1)$ é uma função que depende só de $x$.

Por outro lado o transposto de $L$ é dado por

$$
L^{t}=-\frac{\partial}{\partial t}+i \sum_{j=1}^{n} b_{j}(x, t) \frac{\partial}{\partial x_{j}}+i \sum_{j=1}^{n} \frac{\partial b_{j}(x, t)}{\partial x_{j}}
$$


mas se $x \in \mathcal{N}$ então $\vec{b}(x, t)=\sum_{j=1}^{n} b_{j}(x, t) \frac{\partial}{\partial x_{j}}=0$. Logo $L^{t}$ fica da seguinte forma

$$
L^{t}=-\frac{\partial}{\partial t}+\sum_{j=1}^{n} \frac{\partial b_{j}(x, t)}{\partial x_{j}}
$$

Podemos escrever $\mathcal{N}=\mathcal{N}_{1} \cup \mathcal{N}_{2}$, com

$$
\mathcal{N}_{1}=\left\{x \in \mathcal{N} ; \nabla_{x} \vec{b}(x, t) \neq 0 \text { para algum } t \in(-1,1)\right\}
$$

e $\mathcal{N}_{2}=\mathcal{N} \backslash \mathcal{N}_{1}$. Mostremos que $\mathcal{N}_{1}$ tem medida zero. De fato, seja $x_{0} \in \mathcal{N}_{1}$, então $\nabla_{x} \vec{b}\left(x_{0}, t_{0}\right) \neq 0$, para algum $t_{0} \in(-1,1) . \operatorname{Logo}\left(\partial b_{j} / \partial x_{j}\right)\left(x_{0}, t_{0}\right) \neq 0$ para algum $1 \leq j \leq n$ e, além disso, $b_{j}\left(x_{0}, t_{0}\right)=0$. Pelo Teorema da função implícita temos que existe $\epsilon>0$ tal que o conjunto $\left\{x ; b_{j}\left(x, t_{0}\right)=0\right\} \cap\left\{\left|x-x_{0}\right|<\epsilon\right\}$ é uma hipersuperfície, assim $\rho(x)>0$ q.s em $\left\{\left|x-x_{0}\right|<\epsilon\right\}$. Isto mostra que o conjunto

$$
\{\rho \equiv 0\} \cap\left\{\nabla_{x} \vec{b}(x, t) \neq 0\right\}=\mathcal{N}_{1}
$$

tem medida zero. Portanto $L^{t}=-\frac{\partial}{\partial t}$ sobre $\mathcal{N}_{2} \times(-1,1)$. temos

$$
\begin{aligned}
\iint_{Q} u(x, t) \chi(x) L^{t} \phi(x, t) d x d t & =\int_{\mathcal{N}} \int_{-1}^{1} u(x, t) L^{t} \phi(x, t) d x d t \\
& =-\int_{\mathcal{N}_{2}} u(x) \underbrace{\left(\int_{-1}^{1} \frac{\partial}{\partial t} \phi(x, t) d t\right)}_{0} d x \\
& =0 .
\end{aligned}
$$

Isto mostra (5.5).

Nosso seguinte passo é provar que

$$
\iint u(x, t)(1-\chi(x)) L^{t} \phi d x d t=0 \quad \text { para toda } \phi \in C_{c}^{\infty}(Q) \text {. }
$$

Se $Q_{1}$ é o cubo aberto unitário em $\mathbb{R}^{n}$, ou seja, $Q_{1}=\left\{x \in \mathbb{R}^{n} ;|x|<1\right\}$, vamos construir uma sequência de funções $\phi_{k} \in C_{c}^{\infty}\left(Q_{1} \backslash \mathcal{N}\right)$ tal que $\phi_{k}(x)=1 \operatorname{se} \operatorname{dist}(x, \mathcal{N})>1 / k$ e $\left|\nabla \phi_{k}(x)\right| \leq C k$, onde $C>0$ é uma constante independente de $k$. De fato, seja $\varphi \in C_{c}^{\infty}\left(\mathbb{R}^{n}\right)$ tal que $\int \varphi d x=1$ e $\operatorname{supp}(\varphi) \subseteq B(0,1)$, se $\epsilon>0$, podemos considerar a seguinte função

$$
\varphi_{\epsilon}(x)=\frac{1}{\epsilon^{n}} \varphi\left(\frac{x}{\epsilon}\right) \operatorname{com} \operatorname{supp}\left(\varphi_{\epsilon}\right) \subseteq B(0, \epsilon) \text { e } \int \varphi_{\epsilon} d x=1
$$


Considere também o seguinte

$$
\mathcal{N}_{\epsilon}=\left\{x \in Q_{1} ; \operatorname{dist}(x, \mathcal{N}) \leq \epsilon\right\} \quad \text { e } \quad \psi_{\epsilon}=\chi_{\mathcal{N}_{\epsilon}} * \varphi_{\frac{\epsilon}{2}}
$$

onde $\chi_{\mathcal{N}_{\epsilon}}$ é a função característica do conjunto $\mathcal{N}_{\epsilon}$. Mostremos que se $x \in \mathcal{N}_{\frac{\epsilon}{2}}$, então $\psi_{\epsilon}(x)=1$. Para isto observe que se $x \in \mathcal{N}_{\frac{\epsilon}{2}}$ e $|y|<\epsilon / 2$ então $x-y \in \mathcal{N}_{\epsilon}$ e assim $\chi_{\mathcal{N}_{\epsilon}}(x-y)=1$. Logo temos

$$
\psi_{\epsilon}(x)=\left(\chi_{\mathcal{N}_{\epsilon}} * \varphi_{\frac{\epsilon}{2}}\right)(x)=\int_{|y|<\frac{\epsilon}{2}} \chi_{\mathcal{N}_{\epsilon}}(x-y) \varphi_{\frac{\epsilon}{2}}(y) d y=\int_{|y|<\frac{\epsilon}{2}} \varphi_{\frac{\epsilon}{2}}(y) d y=1 .
$$

Tomemos agora a seguinte sequência de funções, para cada $k \in \mathbb{N}$

$$
\phi_{k}=1-\psi_{\frac{2}{k}}
$$

onde fizemos $\epsilon=2 / k$ em (5.7). Pelo que foi mostrado antes temos que se $x \in \mathcal{N}_{\frac{1}{k}}$ então $\psi_{\frac{2}{k}}(x)=1 \mathrm{e}$, portanto, $\phi_{k}(x)=0$.

Por outro lado se $x \notin \operatorname{supp}\left(\psi_{\frac{2}{k}}\right)$ (i.e. $\left.\operatorname{dist}(x, \mathcal{N})>1 / k\right)$ então

$$
\phi_{k}(x)=1-\underbrace{\psi_{\frac{2}{k}}(x)}_{0}=1 .
$$

Além disso, para qualquer $x \in \mathbb{R}^{n}$, temos que $0 \leq \phi_{k}(x) \leq 1, \phi_{k}(x) \longrightarrow(1-\chi(x))$ quando $k \rightarrow \infty \mathrm{e}$

$$
\begin{aligned}
\left|\frac{\partial}{\partial x_{j}} \psi_{\frac{2}{k}}(x)\right| & =\left|\int_{\mathbb{R}^{n}} \chi_{\mathcal{N}_{\frac{2}{k}}}(y) \frac{\partial}{\partial x_{j}} \varphi_{\frac{1}{k}}(x-y) d y\right|=\left|\int_{\mathcal{N}_{\frac{2}{k}} \cap B\left(0, \frac{1}{k}\right)} \frac{\partial}{\partial x_{j}} \varphi_{\frac{1}{k}}(x-y) d y\right| \\
& \leq \int_{B\left(0, \frac{1}{k}\right)}\left|\frac{\partial}{\partial x_{j}} \varphi_{\frac{1}{k}}(x-y)\right| d y=\int_{B\left(0, \frac{1}{k}\right)}\left|k^{n+1} \frac{\partial}{\partial x_{j}} \varphi(k(x-y))\right| d y \\
& \leq C_{\varphi} \int_{B\left(0, \frac{1}{k}\right)} k^{n+1} d y=C^{\prime} C_{\varphi} \frac{k^{n+1}}{k^{n}}=C k .
\end{aligned}
$$

Disto vem que

$$
\left|\nabla \phi_{k}(x)\right|=\left|\nabla \psi_{\frac{2}{k}}(x)\right| \leq C k
$$

onde a constante $C>0$ é independente de $k$. Logo a sequência $\left\{\phi_{k}\right\}_{k \in \mathbb{N}}$ é a sequência requerida. Agora para mostrar a igualdade (5.6), precisamos do seguinte lema. 
Lema 5.6. Para $k=1,2, \ldots$ temos que

$$
\iint u(x, t) L^{t}\left(\phi_{k} \phi\right) d x d t=0, \quad \text { para toda } \phi \in C_{c}^{\infty}(Q)
$$

Nós deixamos a prova do Lema 5.6 para a próxima seção e vamos supor que (5.8) é satisfeita. Observe como $\phi_{k} \in C_{c}^{\infty}\left(Q_{1} \backslash \mathcal{N}\right)$ e $\phi \in C_{c}^{\infty}(Q)$ então $\phi_{k} \phi \in C_{c}^{\infty}(Q \backslash \mathcal{N} \times(-1,1))$, vamos mostrar que para cada $(x, t) \in Q \backslash \mathcal{N} \times(-1,1)$ temos

$$
u(x, t) L^{t}\left(\phi_{k} \phi\right)(x, t) \longrightarrow u(x, t)(1-\chi(x)) L^{t}(\phi)(x, t), \text { quando } k \rightarrow \infty \text {. }
$$

De fato, se $(x, t) \in Q \backslash \mathcal{N} \times(-1,1)$ então $\chi(x)=0$. Logo, usando a expressão para o transposto de $L$ temos

$$
\begin{aligned}
& u(x, t) L^{t}\left(\phi_{k} \phi\right)(x, t)=u(x, t)[\left.-L\left(\phi_{k} \phi\right)(x, t)+i \phi_{k}(x) \phi(x, t) \sum_{j=1}^{n} \frac{\partial b_{j}(x, t)}{\partial x_{j}}\right] \\
&=u(x, t)\left[-\phi_{k}(x) L(\phi)(x, t)-\phi(x, t) L\left(\phi_{k}\right)(x, t)+\right. \\
&\left.i \phi_{k}(x) \phi(x, t) \sum_{j=1}^{n} \frac{\partial b_{j}(x, t)}{\partial x_{j}}\right]
\end{aligned}
$$

Como $\phi_{k}(x) \longrightarrow(1-\chi(x))$ quando $k \rightarrow \infty$, segue de (5.10) que

$$
\phi_{k}(x) L(\phi)(x, t) \longrightarrow(1-\chi(x)) L(\phi)(x, t)
$$

$\mathrm{e}$

$$
\phi_{k}(x) \phi(x, t) \sum_{j=1}^{n} \frac{\partial b_{j}(x, t)}{\partial x_{j}} \longrightarrow(1-\chi(x)) \phi(x, t) \sum_{j=1}^{n} \frac{\partial b_{j}(x, t)}{\partial x_{j}}
$$

quando $k \rightarrow \infty$. Além disso, como $\phi_{k}(x)=0$ se $x \in \mathcal{N}_{\frac{1}{k}}$ e $\phi_{k}(x)=1$ se $x \notin \operatorname{supp}\left(\psi_{\frac{2}{k}}\right)$, segue que para cada $k \in \mathbb{N}, \operatorname{supp}\left(\nabla \phi_{k}\right) \subset\left\{x \in Q_{1} ; \frac{1}{k} \leq d(x, \mathcal{N}) \leq \frac{2}{k}\right\}$. Logo,

$$
\phi(x, t) L\left(\phi_{k}\right)(x, t)=-i \phi(x, t) \sum_{j=1}^{n} b_{j}(x, t) \frac{\partial \phi_{k}(x)}{\partial x_{j}}=-i \phi(x, t)\left[\vec{b}(x, t) \cdot \nabla \phi_{k}(x)\right] .
$$

Se consideramos $x \in \operatorname{supp}\left(\nabla \phi_{k}\right)$ e aplicamos limite quando $k \rightarrow \infty$ na última expressão acima obtemos que

$$
\phi(x, t) L\left(\phi_{k}\right)(x, t) \longrightarrow 0 .
$$

Portanto, juntando (5.11), (5.12) e (5.13), obtemos (5.9).

Mostremos agora que $u(x, t) L^{t}\left(\phi_{k} \phi\right)$ é limitado. De fato, 


$$
\begin{aligned}
& \left|u(x, t) L^{t}\left(\phi_{k} \phi\right)\right|=\left|u(x, t)\left[-\frac{\partial}{\partial t}+i \sum_{j=1}^{n} b_{j}(x, t) \frac{\partial}{\partial x_{j}}+i \sum_{j=1}^{n} \frac{\partial}{\partial x_{j}} b_{j}(x, t)\right]\left(\phi_{k} \phi\right)\right| \\
& =\mid u(x, t)\left[-\phi_{k}(x) \frac{\partial \phi(x, t)}{\partial t}+i \phi_{k}(x) \sum_{j=1}^{n} b_{j}(x, t) \frac{\partial \phi(x, t)}{\partial x_{j}}+\right. \\
& \left.i \phi(x, t) \sum_{j=1}^{n} b_{j}(x, t) \frac{\partial \phi_{k}(x)}{\partial x_{j}}+i \phi_{k}(x) \phi(x, t) \sum_{j=1}^{n} \frac{\partial b_{j}(x, t)}{\partial x_{j}}\right] \mid \\
& \leq|u(x, t)|\left[\left|\phi_{k}(x) \frac{\partial \phi(x, t)}{\partial t}\right|+\left|\phi_{k}(x) \sum_{j=1}^{n} b_{j}(x, t) \frac{\partial \phi(x, t)}{\partial x_{j}}\right|+\right. \\
& \left.\left|\phi(x, t) \sum_{j=1}^{n} b_{j}(x, t) \frac{\partial \phi_{k}(x)}{\partial x_{j}}\right|+\left|\phi_{k}(x) \phi(x, t) \sum_{j=1}^{n} \frac{\partial b_{j}(x, t)}{\partial x_{j}}\right|\right] \\
& \leq|u(x, t)|\left[\left|\frac{\partial \phi(x, t)}{\partial t}\right|+\left|\sum_{j=1}^{n} b_{j}(x, t) \frac{\partial \phi(x, t)}{\partial x_{j}}\right|+\right. \\
& \left.\left|\phi(x, t) \sum_{j=1}^{n} b_{j}(x, t) \frac{\partial \phi_{k}(x)}{\partial x_{j}}\right|+\left|\phi(x, t) \sum_{j=1}^{n} \frac{\partial b_{j}(x, t)}{\partial x_{j}}\right|\right] .
\end{aligned}
$$

Vamos ver o que acontece com a terceira parcela da expressão (5.14). Temos que $\left|\nabla \phi_{k}(x)\right| \leq$ $C k$ com $C>0$ independente de $k$. Também como $\phi \in C_{c}^{\infty}(Q)$ vem que ela é limitada. Como $\operatorname{supp}\left(\nabla \phi_{k}\right) \subset\left\{x \in Q_{1} ; \frac{1}{k} \leq d(x, \mathcal{N}) \leq \frac{2}{k}\right\}$ e se consideramos $x \in \operatorname{supp}\left(\nabla \phi_{k}\right)$ e $y \in \mathcal{N}$, obtemos

$$
\rho(x)=\rho(x)-\rho(y) \leq|\rho(x)-\rho(y)| \underbrace{\leq}_{5.4}|x-y| \leq \frac{1}{k} .
$$

Portanto disto obtemos o seguinte

$$
\left|\phi(x, t) \sum_{j=1}^{n} b_{j}(x, t) \frac{\partial \phi_{k}}{\partial x_{j}}\right|=\left|\phi(x, t) \vec{b}(x, t) \cdot \nabla \phi_{k}(x)\right| \leq|\phi(x, t)| \rho(x)\left|\nabla \phi_{k}(x)\right| \leq C k \frac{1}{k}=C .
$$

Além disso, como as funções $b_{j}, \phi$ são de classe $C^{\infty}$ então tanto elas quanto suas derivadas são limitadas e por ser $u$ contínua, ela é limitada. Portanto, obtemos que a expressão (5.14) é limitada por uma constante $\tilde{C}>0$ independente de $k$. 
Resumindo temos que

$$
u(x, t) L^{t}\left(\phi_{k} \phi\right)(x, t) \longrightarrow u(x, t)(1-\chi(x)) L^{t}(\phi)(x, t) \quad \text { pontualmente }
$$

$\mathrm{e}$

$$
\left|u(x, t) L^{t}\left(\phi_{k} \phi\right)(x, t)\right| \leq \tilde{C},
$$

logo usando o Teorema da convergência dominada obtemos

$$
\iint u(x, t)\left(1-\chi(x) L^{t} \phi d x d t=\lim _{k \rightarrow \infty} \iint u(x, t) L^{t}\left(\phi_{k} \phi\right) d x d t=0\right.
$$

Isto prova a igualdade (5.6). Finalmente juntando (5.5) e (5.6) obtemos que $L u=0$ no sentido das distribuições em $Q$.

\subsection{Prova do Lema 5.6}

Vamos começar a prova do Lema 5.6. Como $\phi_{k} \phi \in C_{c}^{\infty}(Q \backslash \mathcal{N} \times(-1,1))$, para mostrar a igualdade (5.8) é suficiente mostrarmos que os suportes de $L u$ e $\phi_{k} \phi$ são disjuntos, ou seja, vamos mostrar que $L u=0$ no sentido das distribuições em $Q \backslash(\mathcal{N} \times(-1,1))$ sob a hipotese que $u$ é uma função de Radó. Seja $q \in Q \backslash \mathcal{N} \times(-1,1)$, então $q$ pertence a uma órbita de dimensão dois de $L$ em $Q$, se isto não acontece, como $L$ só tem órbitas de dimensão um e dois, então $q$ teria que estar em uma órbita de dimensão um de $L$ em $Q$ que está contida em $\mathcal{N} \times(-1,1)$, o que implicaria que $q \in \mathcal{N} \times(-1,1)$, que é uma contradição. Da Observação 4.25 vem que em uma vizinhança deste ponto, podemos escolher coordenandas tais que as órbitas de dimensão dois de $L$ são dadas por $x_{j}=C t e$, para $j=2,3, \ldots, n$, isto é, nestas coordenadas o campo $L$ tem a seguinte forma

$$
\frac{\partial}{\partial t}-i b(x, t) \frac{\partial}{\partial x_{1}}, \quad|x| \leq a,|t|<1
$$

$\operatorname{com} b(x, t) \geq 0$. Além disso, satisfaz a seguinte propriedade:

Para todo $|x| \leq a$ a função $(-1,1) \ni t \rightarrow b(x, t)$ não é identicamente zero .

Considerando $x_{2}, \ldots, x_{n}$ como parâmetros e, escrevendo $x$ em lugar de $x_{1}$, podemos então começar com um campo de vetores $L$ em duas variáveis

$$
L=\frac{\partial}{\partial t}-i b(x, t) \frac{\partial}{\partial x}, \quad(x, t) \in \mathbb{R}^{2},
$$


com $b(x, t) \geq 0$ definida em uma vizinhança de $\bar{\Omega}=[-a, a] \times[-T, T]$. Além disso, $L$ satisfaz a condição (P) e pela Observação 5.3, u é ainda uma função de Radó para este campo, vamos assumir também que (5.15) é satisfeita.

Usando o fato que a condição $(\mathrm{P})$ e equivalente à resolubilidade local de $L$ em $C^{\infty}$ e pelo Teorema 4.4, isto implica que $L$ é localmente integrável, logo podemos construir uma função suave $Z$ tal que

$$
L Z=0 \quad \text { e } \quad d Z \neq 0, \text { para todo }(x, y) \in \Omega .
$$

Agora nosso problema é reduzido a provar o seguinte

$$
L u=0 \quad \text { no sentido das distribuições em } \Omega \text {, }
$$

mas, neste caso, $\Omega$ é um subconjunto de $\mathbb{R}^{2}$ dado por $\Omega=(-a, a) \times(-T, T)$. Como falamos antes, como se trata de uma propriedade local, isto pode ser estudado na vizinhança de um determinado ponto $p \in \Omega$. Suponha que $L$ é elítico no ponto $p \in \Omega$, então em coordenadas locais apropriadas temos que $L$ é múltiplo do operador de Cauchy-Riemann (ver Observação 4.5), ou seja,

$$
L=g \frac{\partial}{\partial \bar{z}} \quad \text { numa vizinhança de } p(g \neq 0)
$$

e como $L u=g \partial u / \partial \bar{z}=0$ nos pontos onde $u \neq 0$ (pois $u$ é uma função de Radó para $L$ ), aplicando o Teorema clássico de Radó (Teorema 2.19) vem que

$$
L u=0 \quad \text { na vizinhança de } p .
$$

Assuma agora que $L$ não é elítico em $p$ e, sem perda de generalidade, vamos supor que $p$ é a origem. Ou seja, vamos assumir que $b(0,0)=0$. Seja $\left[t_{0}, t_{1}\right]$ o subintervalo maximal fechado de $[-T, T]$ contendo a origem tal que $b(0, t)=0$, para todo $t \in\left[t_{0}, t_{1}\right]$. Observe que este intervalo pode-se reduzir ao ponto $\{0\}$ mas pela propriedade (5.15), não pode ser igual a $[-T, T]$. Além disso, temos que $L=\partial / \partial t$ e $Z_{x}$ é constante sobre $I=\{0\} \times\left[t_{0}, t_{1}\right]$. De fato, como $b(0, t)$ zera sobre $I, t_{0}<0<t_{1}$ e $b \geq 0$, segue que $b_{x}(0, t)$ zera sobre $I$. Por outro lado, como $L Z=0$, temos que

$$
Z_{t}(x, t)-i b(x, t) Z_{x}(x, t)=0
$$


Derivando a equação (5.17) em relação a $x$ e fazendo $x=0$, obtemos

$$
0=Z_{t x}(0, t)-i \underbrace{b_{x}(0, t)}_{0} Z_{x x}(0, t)=\frac{\partial}{\partial t}\left(Z_{x}(0, t)\right), \text { para todo } t \in\left[t_{0}, t_{1}\right] \text {, }
$$

o qual implica que $Z_{x}$ é constante sobre $I$.

Agora usando $(\operatorname{Re} Z, t)$ como novas coordenadas em uma vizinhança de $I$ (da mesma forma como na Observação 4.5) podemos assumir, depois de redefinir $a$ e $T$, que estamos na seguinte situação

i) $Z(x, t)=x+i \phi(x, t)$ onde $\phi(x, t)$ é uma função suave a valores reais definida para todo $(x, t) \in[-a, a] \times[-T, T]$

ii) $\left|\phi_{x}(x, t)\right|<\frac{1}{2}$, para todo $(x, t) \in[-a, a] \times[-T, T]$;

iii) a função $[-T, T] \ni t \rightarrow \phi(x, t)$ é monótona não decrescente e não constante para qualquer $x \in[-a, a] \mathrm{e}$

iv) $L=\partial / \partial t-B(x, t) \partial / \partial x$, onde $B(x, t)=Z_{t} / Z_{x}=i \phi_{t} /\left(1+i \phi_{x}\right)$.

O item $i$ ) acima se satisfaz porque a função $\phi$ pode ser escolhida de tal forma que $\phi(0,0)=$ $\phi_{x}(0,0)=0$. Seja $R$ um retângulo fechado, com lados paralelos aos eixos coordenados, contido em $[-a, a] \times[-T, T]$ e assuma que $u$ satisfaz $L u=0$ em uma vizinhança de $R$, então pelo Teorema de aproximação de Baouendi-Treves (Teorema 3.88) temos que existe uma sequência de soluções polinomiais $\left\{P_{j}(Z)\right\}_{j \in \mathbb{N}}$ que converge uniformemente a $u$ sobre $R$.

Para concluir a prova de 5.16 precisamos mostrar três lemas que é o que vamos fazer a seguir.

Lema 5.7. A função u é constante sobre as fibras de Z. Além disso, podemos escrever $u=v \circ Z \operatorname{com} v \in C^{0}(Z(\bar{\Omega}))$ unicamente determinada.

Demonstração. Seja $z_{0}=x_{0}+i y_{0}$ fixo e considere a fibra de $Z$ seguinte

$$
F=\left\{(x, t) \in \Omega ; z(x, t)=z_{0}\right\}=\left\{\left(x_{0}, t\right) \in \Omega ; \phi\left(x_{0}, t\right)=y_{0}\right\}
$$

Logo pelo item (iii) acima temos que a fibra de $Z$ tem a seguinte forma

$$
F=\{x\} \times[c, d] \operatorname{com} t \longrightarrow \phi(x, t) \text { constante em }[c, d], \text { para todo } x
$$

Em particular $L=\partial / \partial t$ sobre $F$, pois $\phi_{t} \equiv 0$ nesse conjunto. Então sobre o conjunto aberto $(-a, a) \times(c, d)$, o segmento $\{x\} \times(c, d)$ é uma órbita de dimensão um de $L$ e segue 
do Lema 5.2 que $u$ é constante sobre $\{x\} \times(c, d)$ e, como ela é contínua, segue que também é constante sobre $F$.

Por outro lado, para cada $\xi \in Z(\bar{\Omega})$ definimos a seguinte função

$$
v(\xi)=u(p) \quad \text { se } Z(p)=\xi
$$

Temos que $v$ está bem definida pois $u$ é constante sobre as fibras de $Z$.

Mostremos agora que $v$ é contínua. De fato, seja $\left\{\xi_{n}\right\}_{n \in \mathbb{N}}$ uma sequência em $Z(\bar{\Omega})$ tal que

$$
\xi_{n} \rightarrow \xi^{*} \in Z(\bar{\Omega})
$$

e tomemos uma sequência $\left\{p_{n}\right\}_{n \in \mathbb{N}}$ em $\bar{\Omega} \operatorname{com} Z\left(p_{n}\right)=\xi_{n}$. Como $\bar{\Omega}$ é compacto, passando a uma subsequência se é necessário, podemos assumir que $p_{n} \rightarrow p^{*} \in \bar{\Omega}$. Logo, usando a continuidade de $u$ e $Z$ vem que

$$
v\left(\xi_{n}\right)=u\left(p_{n}\right) \rightarrow u\left(p^{*}\right) \quad \text { e } \quad \xi_{n}=Z\left(p_{n}\right) \rightarrow Z\left(p^{*}\right)
$$

Então pela unicidade de limite temos $Z\left(p^{*}\right)=\xi^{*}$, o que implica que

$$
v\left(\xi^{*}\right)=u\left(p^{*}\right)=\lim _{n \rightarrow \infty} v\left(\xi_{n}\right)
$$

Portanto, $v$ é contínua.

Para a unicidade considere $\tilde{v} \in C^{0}(Z(\bar{\Omega}))$ tal que $u=\tilde{v} \circ Z$ e seja $\xi \in Z(\bar{\Omega})$ com $Z(p)=\xi$. Assim,

$$
\tilde{v}(\xi)=\tilde{v}(Z(p))=u(p)=v(Z(p))=v(\xi)
$$

e, portanto, $\tilde{v}=v$.

Lema 5.8. A função $v$ do Lema 5.7 é holomorfa sobre o conjunto aberto

$$
U=\{\zeta=x+i y ;-a<x<a, \phi(x,-T)<y<\phi(x, T)\}
$$

Demonstração. Pelo Teorema clássico de Radó (Teorema 2.19) só precisamos mostrar que $v$ é holomorfa no conjunto $\tilde{U}=\{\zeta \in U ; v(\zeta) \neq 0\}$. Seja

$$
\zeta=x+i \phi(x, t) \in U \quad \text { e } \quad v(\zeta)=u(x, t)=\lambda \neq 0 .
$$

Se $\phi_{t}(x, t) \neq 0$, então $\phi_{t} \neq 0$ em uma vizinhança de $(x, t)$, pelo item b) da Observação 4.5 
temos que

$$
L=g \frac{\partial}{\partial \bar{\zeta}} \quad \operatorname{com} g \neq 0
$$

Portanto, temos

$$
L u=g \frac{\partial}{\partial \bar{\zeta}} u=0 \quad \Longrightarrow \quad \frac{\partial v}{\partial \bar{\zeta}}=0 \text { numa vizinhança de } \zeta
$$

o qual implica que $v$ é holomorfa em $\tilde{U}$.

Suponha agora que $\phi_{t}(x, t)=0$ e considere a fibra $F$ de $Z$ tal que $(x, t) \in F$. Logo como $u$ é constante sobre $F$ e $u(x, t)=\lambda$, vem que $u=\lambda$ sobre $F$ e $u \neq 0$ em uma vizinhança de $F$. Considere um retângulo fechado $R \operatorname{com} F \subset \stackrel{o}{R}$ tal que

$$
L u=0 \quad \text { em uma vizinhança de } R \text {. }
$$

Observe que a fibra $F$ não toca $\partial \Omega$, pois se isso acontecer pode-se ter, por exemplo, que o ponto $\zeta=x+i \phi(x,-T) \in U$ o qual é absurdo. Logo como consequência do Teorema de aproximação de Baouendi-Treves (Corolário 3.90) temos que existe uma única função $\tilde{v} \in C^{0}(Z(R))$ e holomorfa no interior de $Z(R)$, tal que $u=\tilde{v} \circ Z$ sobre $R$. Agora como $(x, t)$ esta no interior de $R$ e a função $t \longrightarrow \phi(x, t)$ é monotona não decrescente vem que $\zeta$ esta no interior de $Z(R)$, além disso, como $Z(R) \subset Z(\bar{\Omega})$ e pela unicidade de $\tilde{v}$, temos que

$$
\tilde{v}=\left.v\right|_{Z(R)}
$$

o qual mostra que $v$ é holomorfa em $\zeta$ e como $\zeta$ foi tomado arbitrariamente vem que $v$ é holomorfa em $\tilde{U}$. Isto mostra o lema.

Lema 5.9. $L u=0$ no sentido das distribuições em $\Omega$.

Demonstração. Pelos Lemas 5.7 e 5.8, temos que $u=v \circ Z$ com $v$ contínua em $\bar{U}$ e holomorfa em $U$. Logo pelo Teorema de Mergelyan (Teorema 2.18) segue que existe uma sequência de polinômios $P_{j}(\zeta)$ tal que

$$
P_{j} \longrightarrow v \quad \text { uniformemente sobre } \bar{U}
$$

Assim, a sequência de funções $u_{j}=P_{j} \circ Z$ converge uniformemente a $u$. Por outro lado como $L Z=0$ sobre $\Omega$, então para cada $j \in \mathbb{N}$ temos que

$$
\left\langle L u_{j}, \varphi\right\rangle=0, \quad \text { para toda } \varphi \in \mathcal{D}^{\prime}(\Omega),
$$


daí vem que se $\varphi \in \mathcal{D}^{\prime}(\Omega)$

$$
\langle L u, \varphi\rangle=\left\langle u, L^{t} \varphi\right\rangle=\lim _{j \rightarrow \infty}\left\langle u_{j}, L^{t} \varphi\right\rangle=\lim _{j \rightarrow \infty}\left\langle L u_{j}, \varphi\right\rangle=0
$$

Portanto, $L u=0$ no sentido das distribuições em $\Omega$. Isto prova o lema.

Observação 5.10. Observe que o Lema 5.9 implica o Lema 5.6, para mostrar isto considere $\varphi \in C_{c}^{\infty}\left(Q_{n-1}\right)$ uma função nas variáveis $x_{2}, \ldots, x_{n}$ onde $Q_{n-1}$ é o cubo unitário dado por $Q_{n-1}=\left\{x^{\prime}=\left(x_{2}, \ldots, x_{n}\right) ;\left|x^{\prime}\right|<1\right\}$. Pelo Lema 5.9 temos que

$$
\iint u(x, t) L^{t} \phi d x d t=0, \text { para toda } \phi \in C_{c}^{\infty}(\Omega) .
$$

Logo, se multiplicamos a expressão (5.18) pela $\varphi$ e integramos em relação à variável $x^{\prime}$, obtemos

$$
\int \varphi\left(x^{\prime}\right) \iint u(x, t) L^{t} \phi d x d t d x^{\prime}=0 .
$$

Agora, aplicando o Teorema de Fubini e observando que $\varphi L^{t} \phi=L^{t}(\varphi \phi)$, vem que

$$
\iint u(x, t) L^{t}(\varphi \phi) d\left(x, x^{\prime}\right) d t=0 \quad \operatorname{com} \varphi \phi \in C_{c}^{\infty}\left(Q_{n-1}\right) \otimes C_{c}^{\infty}(\Omega)
$$

onde $C_{c}^{\infty}\left(Q_{n-1}\right) \otimes C_{c}^{\infty}(\Omega)$ é o subespaço de $C_{c}^{\infty}\left(Q_{n-1} \times \Omega\right)$ formado por todas as funções $\psi\left(x^{\prime}, y\right)$ que podem ser escritas como somas finitas

$$
\psi\left(x^{\prime}, y\right)=\sum \varphi_{j}\left(x^{\prime}\right) \phi(y), \varphi_{j} \in C_{c}^{\infty}\left(Q_{n-1}\right), \phi \in C_{c}^{\infty}(\Omega)
$$

Finalmente tendo que $C_{c}^{\infty}\left(Q_{n-1}\right) \otimes C_{c}^{\infty}(\Omega)$ é denso em $C_{c}^{\infty}\left(Q_{n-1} \times \Omega\right)$, o resultado segue de (5.19). 


\section{Referências Bibliográficas}

[1] S. Berhanu, P. D. Cordaro, and J. Hounie. An introduction to involutive structures, volume 6 of New Mathematical Monographs. Cambridge University Press, Cambridge, 2008 .

[2] J. B. Conway. Functions of one complex variable, volume 11 of Graduate Texts in Mathematics. Springer-Verlag, New York, second edition, 1978.

[3] P. Cordaro. Sistemas de Campos Vetoriais Complexos. IMPA. Rio de Janeiro, 1986.

[4] I. Glicksberg. Maximal algebras and a theorem of Radó. Pacific J. Math., 14:919-941, 1964.

[5] L. Hörmander. Propagation of singularities and semiglobal existence theorems for (pseudo)differential operators of principal type. Ann. of Math. (2), 108(3):569-609, 1978.

[6] J. Hounie. Teoria Elementar das Distribuições. 12o Colóquio Brasileiro de Matemática. IMPA. Rio de Janeiro, 1979.

[7] J. Hounie and J. Tavares. Radó's theorem for locally solvable vector fields. Proc. Amer. Math. Soc., 119(3):829-836, 1993.

[8] R. Kaufman. A theorem of Radó. Math. Ann., 169:282, 1967.

[9] L. Nirenberg and F. Treves. Solvability of a first order linear partial differential equation. Comm. Pure Appl. Math., 16:331-351, 1963.

[10] J.-P. Rosay and E. L. Stout. Radó's theorem for CR-functions. Proc. Amer. Math. Soc., 106(4):1017-1026, 1989.

[11] W. Rudin. Functional analysis. McGraw-Hill Book Co., New York, 1973. McGrawHill Series in Higher Mathematics. 
[12] W. Rudin. Real and complex analysis. McGraw-Hill Book Co., New York, second edition, 1974. McGraw-Hill Series in Higher Mathematics.

[13] E. L. Stout. A generalization of a theorem of Radó. Math. Ann., 177:339-340, 1968.

[14] H. J. Sussmann. Orbits of families of vector fields and integrability of distributions. Trans. Amer. Math. Soc., 180:171-188, 1973.

[15] F. Trèves. Topological vector spaces, distributions and kernels. Academic Press, New York, 1967. 